\title{
Les lignes directrices du CAD
}

\section{La réduction de la pauvreté}

DÉVELOPPEMENT

\section{OCDE $\ll O$}


(C) OCDE, 2001 .

(C) Logiciel, 1987-1996, Acrobat, marque déposée d'ADOBE.

Tous droits du producteur et du propriétaire de ce produit sont réservés. L'OCDE autorise la reproduction d'un seul exemplaire de ce programme pour usage personnel et non commercial uniquement. Sauf autorisation, la duplication, la location, le prêt, l'utilisation de ce produit pour exécution publique sont interdits. Ce programme, les données y afférantes et d'autres éléments doivent donc être traités comme toute autre documentation sur laquelle s'exerce la protection par le droit d'auteur.

Les demandes sont à adresser au :

Chef du Service des Publications,

Service des Publications de l'OCDE,

2, rue André-Pascal,

75775 Paris Cedex 16, France. 


\section{Les lignes directrices du CAD La réduction de la pauvreté}




\section{ORGANISATION DE COOPÉRATION ET DE DÉVELOPPEMENT ÉCONOMIQUES}

En vertu de l'article $1^{\mathrm{er}}$ de la Convention signée le 14 décembre 1960, à Paris, et entrée en vigueur le 30 septembre 1961, l'Organisation de Coopération et de Développement Économiques (OCDE) a pour objectif de promouvoir des politiques visant :

- à réaliser la plus forte expansion de l'économie et de l'emploi et une progression du niveau de vie dans les pays Membres, tout en maintenant la stabilité financière, et à contribuer ainsi au développement de l'économie mondiale ;

- à contribuer à une saine expansion économique dans les pays Membres, ainsi que les pays non membres, en voie de développement économique ;

- à contribuer à l'expansion du commerce mondial sur une base multilatérale et non discriminatoire conformément aux obligations internationales.

Les pays Membres originaires de l'OCDE sont : l'Allemagne, l'Autriche, la Belgique, le Canada, le Danemark, l'Espagne, les États-Unis, la France, la Grèce, l'Irlande, l'Islande, l'Italie, le Luxembourg, la Norvège, les Pays-Bas, le Portugal, le Royaume-Uni, la Suède, la Suisse et la Turquie. Les pays suivants sont ultérieurement devenus Membres par adhésion aux dates indiquées ci-après : le Japon (28 avril 1964), la Finlande (28 janvier 1969), l'Australie (7 juin 1971), la Nouvelle-Zélande (29 mai 1973), le Mexique (18 mai 1994), la République tchèque (21 décembre 1995), la Hongrie ( 7 mai 1996), la Pologne (22 novembre 1996), la Corée (12 décembre 1996) et la République slovaque (14 décembre 2000). La Commission des Communautés européennes participe aux travaux de l'OCDE (article 13 de la Convention de l'OCDE).

Pour permettre à l'OCDE de réaliser ses objectifs, un certain nombre de comités spécialisés ont été créés. L'un de ceux-ci est le Comité d'Aide au Développement, dont les Membres ont décidé, en commun, de parvenir à un accroissement du volume total des ressources mises à la disposition des pays en développement et d'en améliorer l'efficacité. A cette fin les Membres examinent, ensemble et périodiquement, à la fois le volume et la nature de leurs contributions aux programmes d'aide, établis à titre bilatéral et multilatéral, et se consultent sur toutes les autres questions importantes de leur politique d'aide.

Les Membres du Comité d'Aide au Développement sont : l'Allemagne, l'Australie, l'Autriche, la Belgique, le Canada, le Danemark, l'Espagne, les États-Unis, la Finlande, la France, la Grèce, l'Irlande, l'Italie, le Japon, le Luxembourg, la Norvège, la Nouvelle-Zélande, les Pays-Bas, le Portugal, le Royaume-Uni, la Suède, la Suisse, et la Commission des Communautés européennes.

Also available in English under the title:

The DAC Guidelines

POVERTY REDUCTION

\footnotetext{
(C) Photo : AusAID, Paul Herbert, Australia.

(C) OCDE 2001.

Les permissions de reproduction partielle à usage non commercial ou destinée à une formation doivent être adressées au Centre français d'exploitation du droit de copie (CFC), 20, rue des Grands-Augustins, 75006 Paris, France, tél. (33-1) 440747 70, fax (33-1) 463467 19, pour tous les pays à l'exception des États-Unis. Aux États-Unis, l'autorisation doit être obtenue du Copyright Clearance Center, Service Client, (508)750-8400, 222 Rosewood Drive, Danvers, MA 01923 USA, or CCC Online : www.copyright.com. Toute autre demande d'autorisation de reproduction ou de traduction totale ou partielle de cette publication doit être adressée aux Éditions de l'OCDE, 2, rue AndréPascal, 75775 Paris Cedex 16, France.
} 
LISTE DES SIGLES

RELEVER UN DÉFI MONDIAL : RÉDUIRE LA PAUVRETÉ EN S'APPUYANT SUR LE PARTENARIAT

Déclaration de la réunion à haut niveau du CAD à l'occasion de l'approbation des Lignes directrices du CAD pour la réduction de la pauvreté, les 25 et 26 avril 2001 à Paris

\section{RÉSUMÉ}

Atteindre les objectifs de réduction de la pauvreté à l'échelle mondiale : un appel à l'action

Mettre en œuvre la stratégie du CAD pour le XXI siècle

Des notions et approches communes pour comprendre et combattre la pauvreté Édifier des partenariats efficaces au service de la lutte contre la pauvreté

Cadres et instruments de programmation par pays

La cohérence des politiques est importante Changer notre manière de penser et d'agir

Atteindre les objectifs que nous nous sommes fixés

\section{INTRODUCTION GÉNÉRALE}

La pauvreté dans les pays en développement 33

Une détermination renouvelée, au sein des instances internationales, à faire reculer la pauvreté

Vue d'ensemble des Lignes directrices du CAD pour la réduction de la pauvreté

Objet et vocation des Lignes directrices

Utilisation des Lignes directrices
1. NOTIONS ET APPROCHES

Introduction

Qu'est-ce que la pauvreté ?

La pauvreté, une notion de plus en plus vaste 41

Les principales dimensions de la pauvreté $\quad 42$

Liens entre la pauvreté, la problématique homme-femme et l'environnement

Qui sont les pauvres?

Comment mesurer la pauvreté et en suivre l'évolution?

Comment expliquer la persistance de la pauvreté ?

Quelles sont les mesures à prendre ?

Croissance économique fondée sur une dynamique de réduction de la pauvreté : rythme et qualité

Autonomisation, renforcement des droits et gestion des affaires publiques fondée sur une dynamique de réduction de la pauvreté

Services sociaux de base à l'appui du développement humain

Sécurité humaine : réduire la vulnérabilité et gérer les crises

Prise en compte systématique

de la problématique homme-femme

et promotion de l'égalité homme-femme

Prise en compte systématique de la nécessité d'assurer la pérennité de l'environnement par l'instauration de modes durables d'utilisation des moyens d'existence 


\section{2. ÉDIFIER DES PARTENARIATS POUR LUTTER} CONTRE LA PAUVRETÉ

Introduction

65

Principes fondamentaux de tout partenariat au service de la lutte contre la pauvreté

Les défis du partenariat

Que faut-il pour instaurer de bons partenariats au service de la lutte contre la pauvreté ?

Mesure de la détermination du pays partenaire et des résultats obtenus grâce au partenariat 68

\section{Approches stratégiques de la répartition} d'ensemble de l'aide au développement et de sa programmation

Nécessité d'affecter davantage de ressources aux pays les plus pauvres et aux plus pauvres dans les autres pays en développement

Soutien à des stratégies nationales pertinentes de lutte contre la pauvreté

Le rôle de la coopération pour le

développement selon le contexte national $\quad 71$

Conduite à tenir en cas de problèmes

\section{Aider les partenaires à élaborer} des stratégies nationales valables de lutte contre la pauvreté

Le dialogue sur les politiques à suivre, au cœur de l'interaction entre les partenaires

Soutien des organismes de développement à la conception des stratégies nationales de lutte contre la pauvreté

Renforcement des fondements empiriques des stratégies de lutte contre la pauvreté

Collaboration avec d'autres partenaires

Améliorer la coordination de l'aide à l'appui des stratégies nationales de lutte contre la pauvreté

Priorités d'action des organismes bilatéraux 80
3. CADRES ET INSTRUM ENTS DE PROGRAM MATION PAR PAYS

Introduction

Cadres stratégiques qui se mettent en place dans les pays partenaires

Cadres stratégiques de portée nationale

à l'appui du développement

Stratégie de lutte contre la pauvreté fondée sur l'initiative du pays

Budgets nationaux et examens

des dépenses publiques

Cadres budgétaires à moyen terme

Stratégies par pays élaborées par les organismes d'aide

Domaines sur lesquels doit porter une coopération axée sur la pauvreté

Principaux instruments de coopération financière et technique

Aide-programme

Aide sectorielle

Approches territoriales

Aide-projet

Aide centrée sur la pauvreté et interventions ciblées

Coopération technique à l'appui du renforcement des capacités

Instruments à finalité particulière

Allégement de la dette

Aide en faveur de la prévention des conflits et aide humanitaire

Soutien de la coopération régionale 
4. POUR UNE COHÉRENCE DES POLITIQUES

À L'APPUI DE LA RÉDUCTION

DE LA PAUVRETÉ

105

Introduction

105

Mondialisation et cohérence des politiques

107

Moyens à mettre en œuvre pour améliorer

la cohérence des politiques

Les enseignements de l'expérience

des pays de I'OCDE

Domaines d'action

Commerce international

et investissement direct étranger

Mouvements financiers internationaux

Alimentation et agriculture

116

Ressources naturelles et pérennité

de l'environnement

Questions sociales

Gestion des affaires publiques et conflits

Annexe LISTE INDICATIVE DES CONDITIONS ESSENTIELLES A LA COHÉRENCE DES POLITIQUES À L'APPUI DE LA RÉDUCTION DE LA PAUVRETÉ

Pourquoi centrer les efforts en faveur de la cohérence des politiques sur la lutte contre la pauvreté ?

Pourquoi une liste de conditions essentielles ?

Mesures à prendre pour assurer la cohérence des politiques

La liste indicative, un outil à l'intention des gouvernements désireux de promouvoir la cohérence des politiques au service de la réduction de la pauvreté

5. METTRE LE CHANGEM ENT ET LE DÉVELOPPEM ENT INSTITUTIONNELS AU SERVICE DE LA RÉDUCTION DE LA PAUVRETÉ, DU PARTENARIAT ET DE LA COHÉRENCE DES POLITIQUES Introduction

Pourquoi remettre en cause nos institutions ? 129

Définitions et concepts

Tirer parti au mieux des divers modèles d'organisation

\section{Faire en sorte que tous les services} de l'organisme d'aide tiennent compte de l'objectif de réduction de la pauvreté

Le rôle des responsables de l'organisme d'aide : se donner une ambition, s'engager et créer une culture propice au changement

Mesures à envisager

Rénover les systèmes et les processus institutionnels

Mesures à envisager

Nouvelles capacités

et compétences requises

Mesures à envisager

Structures

Mesures à envisager

Assurer la compatibilité des pratiques de gestion des ressources humaines avec l'objectif de réduction de la pauvreté

Mesures à envisager

Travailler en partenariat

Décentralisation

Mesures à envisager

Alléger le fardeau que font peser les organismes de développement sur leurs partenaires 


\section{Améliorer la capacité institutionnelle de favoriser la cohérence des politiques}

Mesures à envisager

Conclusion

Appendice : OBJECTIFS DE DÉVELOPPEMENT DU MILLÉNAIRE (ODM)

149

\section{TABLEAU}

1. S'attaquer à la problématique de la pauvreté : causes, action entreprise et résultats

\section{FIGURES}

1 Interaction entre les divers aspects de la pauvreté et du bien-être

2 Mesures des différentes dimensions de la pauvreté

3. Mesures permettant d'intégrer progressivement la réduction de la pauvreté et la décentralisation dans un organisme de coopération pour le développement

4. Les cinq structures de base des organismes de coopération pour le développement desMembres du CAD

\section{ENCADRÉS}

1. Une croissance rapide et fondée sur une dynamique de réduction de la pauvreté

2. Programme d'action pour les organismes bilatéraux

3. Liste indicative des conditions essentielles à la cohérence des politiques à l'appui de la réduction de la pauvreté

4. Convergence des politiques intéressant la pauvreté en milieu rural, l'environnement et le développement durable
5. Recommandations pratiques pour un bon fonctionnement des partenariats au service de la lutte contre la pauvreté

6. Évaluer la volonté du gouvernement partenaire de faire reculer la pauvreté

7. Évaluer l'action des organismes de développement en matière de réduction de la pauvreté

8. Comment les organismes bilatéraux peuvent optimiser leur collaboration avec les institutions multilatérales

9. Plan possible d'une stratégie-pays bilatérale axée sur la réduction de la pauvreté

10. L'aide sectorielle au service de la lutte contre la pauvreté : le cas du secteur de l'éducation en Ouganda

11. Promouvoir des systèmes de santé favorables aux pauvres

12. Axer les projets d'infrastructure sur la réduction de la pauvreté

13. Les différents aspects de la cohérence des politiques

14. Liste indicative des conditions essentielles à la cohérence des politiques à l'appui de la réduction de la pauvreté

15. Mesures prises au Royaume-Uni en faveur de la cohérence des politiques

16. Cohérence des politiques : domaines d'action

17. Les différents aspects de la cohérence des politiques

18. Cohérence des politiques :

liste élargie des domaines d'action

19. Quelques conseils pour une décentralisation réussie 


\section{Liste des sigles}

$\begin{array}{ll}\text { ACDI } & \text { Agence canadienne de développement international } \\ \text { ADPIC } & \text { Aspects des droits de propriété intellectuelle qui touchent au commerce } \\ \text { AGCS } & \text { Accord général sur le commerce des services } \\ \text { APD } & \text { Aide publique au développement } \\ \text { Asdi } & \text { Agence suédoise de coopération pour le développement international } \\ \text { BMZ } & \text { Ministère fédéral de la Coopération et du Développement économiques (Allemagne) } \\ \text { CAD } & \text { Comité d'aide au développement } \\ \text { CCI } & \text { Centre du commerce international } \\ \text { CDI } & \text { Cadre de développement intégré } \\ \text { CE } & \text { Commission européenne (assure la gestion du programme d'aide de la Communauté }\end{array}$

CNUCED Conférence des Nations unies sur le commerce et le développement

CNUED Conférence des Nations unies sur l'environnement et le développement

CSLP Cadre stratégique de lutte contre la pauvreté

CT Coopération technique

DFID Department for International Development (Royaume-Uni)

DPI Droits de propriété intellectuelle

FIDA Fonds international de développement agricole

FMI Fonds monétaire international

GC Groupe consultatif

GTZ Agence allemande de coopération technique

IDE Investissement direct étranger

IDEA Institut pour la démocratie et l'assistance électorale

IDH Indicateur du développement humain

IPF Indicateur de la participation des femmes

IPH-1 Indice de la pauvreté humaine (pays en développement)

IPH-2 Indice de la pauvreté humaine (pays de l'OCDE et en transition)

ISDH Indicateur sexospécifique du développement humain

OCDE Organisation de coopération et de développement économiques

ODM Objectifs de développement du millénaire

OGM Organisme génétiquement modifié

OID Objectifs internationaux de développement

OIT Organisation internationale du travail

OMC Organisation mondiale du commerce

OMS Organisation mondiale de la santé

ONG Organisation non gouvernementale

ONUSIDA Programme des Nations unies sur le VIH/sida

OSC Organisations de la société civile

PIB Produit intérieur brut

PMA Pays les moins avancés

PNUD Programme des Nations unies pour le développement

PPA Parité de pouvoir d'achat

PPTE Pays pauvre très endetté

PSA Partenariat stratégique pour l'Afrique

SE-AGNU Session extraordinaire de l'Assemblée générale des Nations unies 
SGP Système généralisé de préférences

sida Syndrome d'immunodéficience acquise

SLP Stratégie de lutte contre la pauvreté

SNDD Stratégie nationale de développement durable

TIC Technologies de l'information et des communications

UE Union européenne

UNDAF Plan-cadre des Nations unies pour l'aide au développement

UNESCO Organisation des Nations unies pour l'éducation, la science et la culture

UNIFEM Fonds de développement des Nations unies pour la femme

USAID Agence des États-Unis pour le développement international

VIH Virus d'immunodéficience humaine 


\section{RELEVER UN DÉFI MONDIAL : RÉDUIRE LA PAUVRETÉ EN S'APPUYANT SUR LE PARTENARIAT}

\section{Déclaration de la réunion à haut niveau du CAD à l'occasion de l'approbation des Lignes directrices du CAD pour la réduction de la pauvreté, les 25 et 26 avril 2001 à Paris}

L es pays en développement ont obtenu des avancées remarquables, encore qu'inégales, dans l'amélioration de leur niveau de vie au cours des trente dernières années, et la coopération pour le développement y a grandement contribué. La réduction de la pauvreté dans le cadre d'un développement durable demeure néanmoins un défi majeur. L'extrême pauvreté est encore le lot d'un habitant sur quatre du monde en développement. L'illettrisme, la faim et la maladie restent des maux largement répandus, et le $\mathrm{VIH} /$ sida est devenu un véritable fléau dans de nombreux pays en développement. La moitié environ des pauvres sont des enfants, exposés aux privations, au dénuement et à la violence - et la majorité des adultes recensés comme pauvres sont des femmes. Les inégalités sociales et économiques au sein des nations constituent autant d'obstacles à un recul durable de la pauvreté. La mondialisation offre des perspectives prometteuses pour stimuler la croissance et faire reculer la pauvreté, mais un effort particulier devra être fait pour s'assurer que les pays et les populations pauvres reçoivent leur juste part des opportunités et des avantages qui en découlent.

Le sort des personnes qui vivent dans une profonde pauvreté est pour nous un grave sujet de préoccupation. Au-delà du sens moral partagé qui nous incite à aider les plus défavorisés, nous estimons que la réduction de la pauvreté et des inégalités dans le monde participe de notre intérêt commun, compte tenu de l'impact à en attendre sur la sécurité régionale et mondiale, la coopération internationale, le développement durable et la prospérité. C'est toutefois aux pays en développement de prendre la conduite des opérations et de formuler des stratégies nationales efficaces de lutte contre la pauvreté. Ces stratégies doivent inscrire les préoccupations économiques, sociales, environnementales ainsi que les questions de gouvernance dans un cadre de développement intégré, d'envergure nationale. Pour notre part, nous nous engageons, en partenariat avec la société civile, le secteur privé et les organisations multilatérales, à les aider à se monter à la hauteur de l'enjeu. Nous nous engageons en outre à ne ménager aucun effort pour aider les pays en développement à faire face aux défis de la mondialisation et de l'ère numérique, et à s'attaquer au VIH/sida et à toutes les autres maladies qui constituent encore un péril mortel.

Nous confirmons notre volonté de réduire la pauvreté, sous toutes ses facettes, et d'assurer la réalisation des sept Objectifs internationaux de développement (OID). Nous concevons les OID dans l'ensemble plus large 
d'objectifs communs - concernant notamment la lutte contre la faim, l'accès à l'eau potable et la lutte contre le VIH/sida - définis dans la Déclaration du Millénaire des Nations unies ainsi que dans la perspective à terme d'éradiquer la pauvreté. Ces OID sont les suivants : pour 2015, réduire de moitié l'extrême pauvreté monétaire, faire reculer la mortalité infantile, juvénile et maternelle, assurer une éducation primaire à tous les enfants et mettre les services de santé génésique à la disposition de tous ; pour 2005, éliminer les disparités entre les sexes dans l'enseignement afin de contribuer à l'égalité homme-femme et au renforcement des moyens d'action des femmes, et mettre en œuvre des stratégies de développement durable afin d'inverser la tendance à la déperdition des ressources environnementales.

Nous réexprimons notre détermination à promouvoir les aspects qualitatifs du développement - notamment une gestion démocratique, efficace et transparente des affaires publiques, la protection des droits de l'homme et le respect de l'état de droit - en soutenant les efforts que déploient nos partenaires en développement pour bâtir des sociétés stables, sûres, justes et fondées sur la participation. Nous sommes déterminés à faire de la réduction durable de la pauvreté l'axe essentiel de notre action de coopération pour le développement, en particulier à l'échelon local. Nous sommes en outre résolus à renforcer la cohérence de l'ensemble de nos politiques ayant des retombées sur le développement, par exemple en ouvrant davantage nos marchés et en accélérant la mise en œuvre des allégements de dette. Nous redoublerons d'efforts pour améliorer l'efficacité de l'aide et mobiliser des ressources supplémentaires au service de la lutte contre la pauvreté. Sur ce plan, la plupart des Membres sont guidés par l'objectif de $0.7 \%$ fixé pour le rapport APD/PNB.

Nous avons élaboré des Lignes directrices pour la réduction de la pauvreté, fruit de consultations avec nos partenaires internationaux, à savoir la Banque mondiale, le Fonds monétaire international et le Programme des Nations unies pour le développement. Ces Lignes directrices attestent d'un consensus international naissant, d'une volonté partagée et d'une perception commune des moyens qui s'offrent à nous d'aider, ensemble, de façon plus efficace, nos partenaires en développement à faire reculer la pauvreté. Nous souscrivons aux principes suivants, pierres angulaires de ces Lignes directrices.

\section{La pauvreté est pluridimensionnelle}

Nous partageons une même approche de la pauvreté et de ses multiples dimensions.
La notion de pauvreté recouvre différentes dimensions du dénuement liées à l'incapacité de satisfaire des besoins humains tels que consommer et assurer sa sécurité alimentaire, être en bonne santé, pouvoir apprendre, pouvoir faire valoir ses droits et entendre sa voix, vivre en sécurité et dans la dignité, et exercer un travail décent. La pauvreté doit également être combattue en vertu de considérations liées à la protection de l'environnement et à l'instauration d'un développement durable. Enfin, la réduction des 
inégalités entre hommes et femmes est un élément incontournable, quelle que soit la dimension de la pauvreté envisagée.

\section{La cohérence de nos politiques est de première importance}

La réduction de la pauvreté appelle une plus grande cohérence des politiques gouvernementales susceptibles d'affecter le développement. L'allégement de la dette, les échanges, l'investissement, l'agriculture, l'environnement, les migrations, la recherche sur la santé, la sécurité et les ventes d'armes comptent parmi les principaux domaines dans lesquels une action des pouvoirs publics peut avoir un impact notable sur la réduction de la pauvreté.

\section{Croissance économique : rythme, qualité et équité}

La réduction de la pauvreté passe par une croissance rapide et durable, favorable aux pauvres. Cela suppose une bonne gestion des affaires publiques, une gestion macro-économique prudente, des marchés concurrentiels et un secteur privé dynamique, des institutions efficientes et des modes durables d'exploitation des ressources naturelles. Rendre la croissance favorable aux pauvres implique une participation équitable des pauvres, hommes et femmes, à la production et aux fruits de cette croissance. Cela nécessite aussi des réformes pour réduire les inégalités liées aux capacités humaines et à l'accès à des actifs et des ressources productives comme la terre, la formation et le crédit.

\section{Réduire la pauvreté exige une volonté politique de la part de tous les partenaires}

Toute stratégie de réduction de la pauvreté relève d'un processus politique. Elle suppose une action résolue pour démarginaliser les pauvres en leur donnant des moyens de se faire entendre et en favorisant les principes démocratiques de reddition des comptes. L'instauration de partenariats stratégiques avec des forces acquises à la réforme et la société civile peut utilement contribuer à la transformation sociale et politique. L'action engagée en faveur d'un élargissement du dialogue à l'échelon local et de la participation des parties prenantes doit aller dans le sens des efforts que déploient nos partenaires pour mettre en place des institutions démocratiques.
Nous nous appliquerons à renforcer la cohérence de nos politiques au service de la réduction de la pauvreté dans nos décisions gouvernementales, et à nous doter des moyens nécessaires pour la promouvoir au sein de nos gouvernements et des instances internationales.

Nous soutiendrons les efforts que déploient nos partenaires pour promouvoir une croissance durable favorable aux pauvres, réduire les inégalités et accroître leur part des courants mondiaux d'échange et d'investissement.

Nous soutiendrons les initiatives des pays partenaires visant à associer la société civile à la fixation des objectifs prioritaires de réduction de la pauvreté et des mesures destinées à atténuer les inégalités, dans le cadre des efforts déployés par ces pays pour bâtir des institutions démocratiques. 
Nous accorderons la priorité aux pays pauvres dont le gouvernement se montre déterminé à lutter contre la pauvreté et qui utilise efficacement I'aide, mais nous ciblerons également notre aide, de manière sélective, sur les pauvres vivant dans des pays où la gestion des affaires publiques soulève de graves problèmes.

Nous recourrons à davantage de coordination entre nos aides - programmes, soutiens sectoriels et projets - afin d'appuyer la mise en œuvre de stratégies et programmes mis en place par les pays pour lutter contre la pauvreté.

Nous ne ménagerons aucun effort pour favoriser la collaboration, simplifier nos procédures administratives lorsque ce sera possible, et coordonner nos approches et nos actions avec celles de nos partenaires.

\section{Les besoins exprimés et les résultats obtenus seront des critères déterminants dans la répartition de l'aide}

Les ressources allouées à la coopération pour le développement doivent être utilisées efficacement au service de la réduction de la pauvreté. Priorité sera donnée aux pays à faible revenu. Une aide ciblée pourra également être accordée à d'autres pays dont une forte proportion de la population est pauvre. La volonté politique manifestée à l'égard de la lutte contre la pauvreté et l'efficacité des politiques gouvernementales seront déterminantes à cet égard. Cela dit, il est également important d'aider les pauvres des pays aux prises avec de graves difficultés sur le plan de la gestion publique, y compris les pays particulièrement exposés aux conflits.

\section{Soutenir les stratégies de lutte contre la pauvreté de nos partenaires au moyen de différents types d'aide}

La coopération pour le développement sera mise au service des objectifs et priorités définis dans les stratégies nationales de réduction durable de la pauvreté, lesquelles doivent relever de l'initiative du pays lui-même, s'inscrire dans une démarche participative, présenter un caractère intégré, et être axées sur les résultats. Afin de favoriser l'appropriation et la durabilité, la communauté du développement doit veiller à ce que les activités entreprises répondent à une demande des pays intéressés au lieu d'être déterminées par les organismes d'aide. Cela permettra d'instaurer des partenariats fondés sur une approche intégrée des appuis aux programmes, secteurs et projets. Pour soutenir la mise en œuvre de stratégies arrêtées par nos partenaires, il est prioritaire de mobiliser des ressources à l'appui du renforcement des capacités, de la réforme des institutions et d'une large participation des partenaires locaux.

\section{Une meilleure gestion de l'aide pour une efficacité accrue}

L'efficacité de l'aide passe par une meilleure coordination, grâce à la collaboration, à la réalisation d'activités conjointes et à la mise en commun des compétences et des ressources. Tout en maintenant des normes élevées de transparence et de responsabilité, les Membres du CAD peuvent simplifier et harmoniser leurs exigences administratives et financières, s'adapter aux procédures locales lorsque celles-ci sont satisfaisantes et aider leurs partenaires à améliorer leurs capacités et leur performance administratives. 


\section{Évaluation des performances}

Pour être solides, les partenariats doivent se fonder sur le dialogue, la confiance mutuelle et la responsabilité conjointe. L'action de chaque partenaire doit être évaluée au regard des engagements convenus et de son impact sur la réduction de la pauvreté. Les organismes de développement doivent mettre au point des objectifs précis de performance en matière de partenariat, par exemple promouvoir l'appropriation locale, assouplir les modalités de transfert des ressources, rendre plus prévisible le montant de ces dernières, allonger le cycle de leur programmation, et veiller à ce que l'aide au développement consentie au secteur public se reflète intégralement dans les budgets des pays partenaires.

\section{Mieux aligner la structure institutionnelle des organismes d'aide sur les objectifs de réduction de la pauvreté, de partenariat et de cohérence des politiques}

Faire de la lutte contre la pauvreté une priorité essentielle pour tous les services, œuvrer au renforcement des capacités de partenariat et promouvoir la cohérence des politiques constituent autant d'objectifs fondamentaux, au plan institutionnel, pour les organismes d'aide. Cela nécessitera souvent des changements et des innovations au niveau des structures organiques, des pratiques, des systèmes d'incitation et des cultures institutionnelles.

Nous procéderons à une évaluation de nos efforts de coopération pour le développement afin de nous assurer qu' ils contribuent efficacement à promouvoir un véritable partenariat et qu'ils ont un réel impact sur la réduction de la pauvreté.

Nous sommes résolus à intégrer la lutte contre la pauvreté et le principe de partenariat dans les politiques et activités de nos organismes d'aide, et nous nous efforcerons d'adapter en conséquence nos pratiques, systèmes et cultures institutionnels. 


\section{Atteindre les objectifs de réduction de la pauvreté à l'échelle mondiale : un appel à l'action}

Depuis plusieurs décennies, la communauté de l'aide au développement travaille en collaboration avec les populations et les gouvernements des pays en développement pour améliorer leurs conditions de vie. Les progrès réalisés ont été remarquables, même s'ils ont reçu peu d'écho en général. Dans les trente dernières années seulement, l'espérance de vie s'est allongée de plus de vingt ans (passant à 62 ans), le taux de mortalité infantile a été divisé par deux et le taux de scolarisation dans le primaire a doublé. De grands pays en développement, d'Asie de l'Est en particulier, sont passés du statut de pays à faible revenu à celui de pays à revenu intermédiaire.

Autant de résultats très encourageants, qui montrent que la pauvreté peut être vaincue. Mais la bataille est loin d'être gagnée. Une personne sur quatre (soit 1.2 milliard d'individus) vit encore dans l'extrême pauvreté monétaire dans les pays en développement ${ }^{1}$ - ou une personne sur cinq dans le monde - et les progrès face à ce fléau ont été inégaux. L'Asie, par exemple, malgré des avancées rapides, compte encore le plus grand nombre de pauvres dans le monde. En Afrique subsaharienne, à la lenteur de la croissance s'est conjuguée une augmentation de la pauvreté, sous l'effet en partie de conflits et de problèmes de gouvernance, et cette région est aujourd'hui confrontée au fléau du $\mathrm{VIH} /$ sida.

De nouvelles menaces se dessinent. Les inégalités sociales et économiques au sein des nations font obstacle à une réduction durable de la pauvreté. En outre, la marginalisation des minorités, ethniques notamment, reste source de conflits violents. Les pauvres demeurent exclus de la vie économique et politique dans de nombreux pays, ainsi que de la grande vague de la mondialisation. Les défis et les enjeux associés à l'éradication de la pauvreté sont élevés - et prennent une acuité de plus en plus grande.

L'évolution de la dynamique mondiale ajoute de nouvelles dimensions, inquiétantes, à la notion de pauvreté. L'accélération de l'intégration économique entre nations va alimenter la croissance du revenu et de l'emploi, qui encouragera la mise en place de nouveaux modèles de production et d'échanges et créera des opportunités inégalées d'apprentissage, de communication et de partage du savoir. La mondialisation ouvre d'immenses perspectives d'accès à l'autonomie et de promotion de la compréhension mutuelle, des relations et des partenariats entre les pays. Mais elle menace aussi de creuser le fossé entre riches et pauvres, et d'accroître le risque de voir s'instaurer un monde à deux vitesses, où certains pays ou régions seront de plus en plus à la traîne. La mondialisation ne livrera pas ses bienfaits potentiels si elle ne profite qu'à quelquesuns.

Cependant, dans le contexte actuel de mondialisation rapide, les maux sociaux associés à la pauvreté - la maladie, les migrations illicites, la dégradation de l'environnement, la délinquance, l'instabilité politique, les conflits armés et le terrorismepeuvent se propager facilement et en toute impunité au-delà des frontières et des continents. Il faut aussi compter avec la pression de la croissance démographique. Les

\section{L'éradication de la pauvreté est essentielle à la sécurité et à la prospérité dans le monde...}

tangibles ont été obtenues dans le monde en développement grâce aux efforts conjoints des gouvernements, de la société civile et des organismes d'aide au développement...

....mais l'extrême pauvreté est encore le lot d' une personne sur quatre. 
...et chacun a donc intérêt à la combattre.
Stimulés par la stratégie définie par le CAD et I'OCDE dans « Le rôle de la coopération pour le développement à $I^{\prime}$ aube du XXI siècle $" . .$.
2 milliards d'individus qui, selon les estimations, viendront s'ajouter à la population actuelle dans les vingt prochaines années, vivront à $97 \%$ dans les pays en développement. L'éradication de la pauvreté ne répond donc pas uniquement à un souci moral et humanitaire, elle est essentielle à la sécurité et à la prospérité dans le monde, ainsi qu'à la réduction des pressions qui pèsent sur l'environnement. Il s'agit d'un bien collectif de premier ordre répondant aux intérêts de l'ensemble de la population mondiale.

La conjoncture actuelle est favorable à la lutte contre la pauvreté. Il y a aujourd'hui à l'échelle mondiale une réelle volonté de réduire de moitié d'ici 2015 la proportion d'individus vivant dans une extrême pauvreté monétaire et souffrant de la faim. Les pays en développement élaborent et mettent en œuvre des stratégies à cette fin. Quant à la communauté du développement, elle œuvre pour apporter une réponse ciblée à ce problème, en faisant preuve de la volonté politique et en mettant en place les cadres et mécanismes nécessaires pour organiser une action concertée et efficace de lutte contre la pauvreté.

C'est le moment de mettre à profit ces circonstances particulières : une mobilisation grandissante en faveur de la lutte contre la pauvreté, les possibilités offertes à tous de tirer parti de la mondialisation et les avancées technologiques dans le domaine des télécommunications, de l'information et des sciences de la vie. Il faut être à la hauteur de nos promesses, de nos convictions et de nos objectifs, et donner corps à cet engagement grâce à des ressources et à des efforts avisés sur le terrain. Chacun a quelque chose à gagner à travailler plus efficacement, en élargissant son champ d'action, pour combattre la pauvreté dans le monde.

\section{Mettre en œuvre la stratégie du CAD pour le XXIe siècle}

La stratégie du CAD de l'OCDE énoncée dans Le rôle de la coopération pour le développement à l'aube $d u X X I^{e}$ siècle présente une conception de la coopération pour le développement qui repose sur le principe de partenariats construits autour de stratégies de développement prises en charge et pilotées par les gouvernements et la société civile des pays en développement. Les principes qui sous-tendent cette conception - le partenariat, la prise en main à l'échelon local, la conduite des opérations par le pays concerné, une large participation, l'efficacité du développement et l'obligation de rendre des comptes - ont des conséquences de vaste portée sur la façon dont opèrent les organismes de développement. Ces derniers doivent mettre en place une collaboration bien plus étroite et coordonnée avec un plus large éventail de partenaires ${ }^{2}$. Ils doivent apporter une aide adaptée aux besoins et aux priorités du pays partenaire, lorsque les conditions d'un partenariat sont remplies. Ils ont maintenant l'obligation de rendre des comptes aux partenaires et à leur propre opinion publique quant aux actions menées et aux engagements pris. Ils doivent jouer un rôle de soutien plutôt que de déclencheur du développement.

Par la stratégie pour le XXI ${ }^{\mathrm{e}}$ siècle, les Membres du CAD se sont aussi engagés à faciliter un recul de la pauvreté dans les pays en développement en aidant ces derniers à atteindre un ensemble précis d'objectifs en matière de développement économique et social et de durabilité environnementale - les objectifs internationaux de développement (OID) - se fondant sur les accords conclus lors des grandes manifestations internationales organisées au cours des années 90. Les Membres du CAD sont également convenus d'insister sur les facteurs qualitatifs qui contribueront à l'instauration de sociétés plus stables, plus sûres, plus justes et davantage fondées sur la participation, à leurs yeux essentielle pour la réalisation de ces objectifs mesurables. Parmi ces facteurs figurent notamment la capacité d'assurer une gestion efficace, démocratique et responsable des affaires publiques, la protection des droits de l'homme et le respect de l'État de droit. 
Objectifs de développement du millénaire (ODM)

\section{OBJ ECTIF 1. Éliminer l'extrême pauvreté et la faim}

1. Réduire de moitié, entre 1990 et 2015, la proportion de la population dont le revenu est inférieur à un dollar par jour

2. Réduire de moitié, entre 1990 et 2015 , la proportion de la population qui souffre de la faim

\section{OBJECTIF 2. Assurer une éducation primaire pour tous}

3. D'ici à 2015, donner à tous les enfants, garçons et filles, partout dans le monde, les moyens d'achever un cycle complet d'études primaires

\section{OBJECTIF 3. Promouvoir l'égalité des sexes et l'autonomisation des femmes}

4. Éliminer les disparités entre les sexes dans les enseignements primaire et secondaire d'ici à 2005 si possible, et à tous les niveaux de l'enseignement en 2015 au plus tard

\section{OBJ ECTIF 4. Réduire la mortalité des enfants de moins de 5 ans}

5. Réduire de deux tiers, entre 1990 et 2015, le taux de mortalité des enfants de moins de 5 ans

\section{OBJ ECTIF 5. Améliorer la santé maternelle}

6. Réduire de trois quarts, entre 1990 et 2015, le taux de mortalité maternelle

\section{OBJ ECTIF 6. Combattre le VIH/ sida, le paludisme et d'autres maladies}

7. D'ici à 2015, avoir stoppé la propagation du VIH/sida et commencé à inverser la tendance actuelle

8. D'ici à 2015, avoir maîtrisé le paludisme et d'autres grandes maladies, et avoir commencé à inverser la tendance actuelle

\section{OBJ ECTIF 7. Assurer un environnement durable}

9. Intégrer les principes du développement durable dans les politiques nationales et inverser la tendance actuelle à la déperdition des ressources environnementales

10. Réduire de moitié, d'ici à 2015, le pourcentage de la population qui n'a pas accès de façon durable à un approvisionnement en eau potable salubre

11. Réussir, d'ici à 2020, à améliorer sensiblement la vie d'au moins 100 millions d'habitants de taudis

\section{OBJECTIF 8. Mettre en place un partenariat mondial pour le développement}

12. Poursuivre la mise en place d'un système commercial et financier multilatéral ouvert, fondé sur des règles, prévisible et non discriminatoire. (Cela suppose un engagement en faveur d'une bonne gouvernance, du développement et de la lutte contre la pauvreté, aux niveaux tant national qu'international)

13. S'attaquer aux besoins particuliers des pays les moins avancés. (La réalisation de cet objectif suppose l'admission en franchise et hors contingents des produits exportés par les pays les moins avancés ; l'application du programme renforcé d'allégement de la dette des PPTE et l'annulation des dettes publiques bilatérales ; et l'octroi d'une APD plus généreuse aux pays qui démontrent leur volonté de lutter contre la pauvreté)

14. Répondre aux besoins particuliers des petits États insulaires en développement (en appliquant le Programme d'action pour le développement durable des petits États insulaires en développement et les conclusions de la $22^{\mathrm{e}}$ session extraordinaire de l'Assemblée générale)

15. Traiter globalement le problème de la dette des pays en développement, par des mesures d'ordre national et international propres à rendre leur endettement viable à long terme

16. En coopération avec les pays en développement, formuler et appliquer des stratégies qui permettent aux jeunes de trouver un travail décent et utile

17. En coopération avec l'industrie pharmaceutique, rendre les médicaments essentiels disponibles et abordables dans les pays en développement

18. En coopération avec le secteur privé, faire en sorte que tous bénéficient des avantages des nouvelles technologies, en particulier des technologies de l'information et de la communication.

La Déclaration du millénaire des Nations unies, adoptée par les Chefs d'État et de gouvernement en septembre 2000, énonce quant à elle un ensemble plus large d'objectifs quantitatifs et qualitatifs de développement destinés à mesurer les progrès accomplis vers le but suprême : l'éradication de la pauvreté. Ces objectifs mesurables reprennent et actualisent les OID qui avaient au départ été fixés dans la stratégie du CAD/OCDE 
....les organismes bilatéraux ont élaboré les présentes Lignes directrices destinées à rehausser l'efficacité de leur action au service de la lutte contre la pauvreté. pour le XXI ${ }^{\mathrm{e}}$ siècle. Ces objectifs de développement dits « du millénaire » (ODM) sont décrits ci-dessus ${ }^{3}$.

Sous l'impulsion de la stratégie pour le XXI ${ }^{\mathrm{e}}$ siècle, les Membres du CAD s'engagent avec une détermination plus grande encore à œuvrer au recul de la pauvreté, tant par souci de solidarité avec les plus pauvres que par volonté de faire respecter les droits universels de l'être humain. Ils s'appliqueront à faire de la réduction durable de la pauvreté l'objectif central de la coopération pour le développement et à intégrer les aspects économiques, sociaux et environnementaux ainsi que les questions de gouvernance dans une approche globale du développement à l'échelon de chaque pays.

Déterminés à travailler plus efficacement à réduire la pauvreté, les Membres du CAD ont élaboré un ensemble de lignes directrices en vue de coordonner et d'améliorer leurs efforts, individuels et collectifs. Les Lignes directrices $d u$ CAD pour la réduction de la pauvreté couvrent cinq grands thèmes : les notions et approches relatives à la pauvreté, les questions de partenariat, la programmation par pays, la cohérence des politiques et les changements institutionnels au sein des organismes de développement. Ce résumé met en relief les principales conclusions, engagements et enjeux qui en ressortent.

\section{Encadré 1. Une croissance rapide et fondée sur une dynamique} de réduction de la pauvreté

Une croissance économique vigoureuse et soutenue du secteur privé est créatrice d'emplois et de revenus pour les pauvres. Elle est aussi génératrice de recettes publiques susceptibles de financer des programmes de développement social et de protection sociale, et un renforcement du cadre institutionnel et des infrastructures matérielles nécessaires au bon fonctionnement des marchés. L'État, le secteur privé et la société civile ont tous un rôle crucial à jouer dans la réduction de la pauvreté en encourageant une croissance économique favorable aux pauvres, par l'instauration de marchés efficaces et concurrentiels. Toutefois, même vigoureuse et durable, la croissance peut faire des laissés-pour-compte. La croissance $\mathrm{du}$ PIB n'explique qu'environ la moitié seulement de l'accroissement des revenus du cinquième le plus pauvre de la population. L'autre moitié vient de la qualité de la croissance, de sa composition, de sa répartition et de son caractère durable.

Comment fonder davantage la croissance sur une dynamique de réduction de la pauvreté ? En adoptant des politiques et programmes qui permettent aux pauvres d'accéder à des actifs humains, matériels et financiers susceptibles de contribuer à accroître leur productivité et leurs revenus et qui visent, par exemple, à renforcer les services sociaux (en particulier dans les domaines de l'éducation et de la santé), à réformer le système foncier ou à mettre en place des dispositifs de micro-crédit. Encourager les petites exploitations agricoles et les activités manufacturières à forte intensité de main-d'œuvre et mettre en place les institutions et les infrastructures nécessaires à cet effet est également essentiel pour l'instauration d'une croissance favorable aux pauvres. A un niveau plus général, une bonne gouvernance, notamment une gestion macro-économique prudente et un faible taux d'inflation, et des capacités institutionnelles satisfaisantes, notamment des cadres bien conçus pour les marchés financiers et les entreprises, revêtent aussi une importance primordiale.

\section{Des notions et approches communes pour comprendre et combattre la pauvreté}

Une réduction durable de la pauvreté nécessite des stratégies efficaces reposant sur des notions et des approches cohérentes et précises. Différentes perceptions de la pauvreté conduisent à différentes manières de s'y attaquer. Partager une même conception, claire, de la pauvreté permet d'élaborer un programme d'action commun avec les partenaires, en associant aux causes spécifiques de la pauvreté dans chaque contexte des mesures et des actions appropriées. Les étapes qui suivent constituent le fondement de la démarche à adopter pour déterminer la stratégie de lutte contre la pauvreté à retenir dans chaque pays : 
Identifier les principales causes de la pauvreté.

Élaborer et hiérarchiser des politiques et des mesures visant à y remédier.

Définir des indicateurs ou des objectifs pour le suivi des progrès réalisés.

- Rechercher un accord général sur les politiques et les programmes de lutte contre la pauvreté.

La pauvreté est pluridimensionnelle. La pauvreté traduit l'impossibilité d'accéder à des conditions de vie socialement adéquates et recouvre diverses formes de privation. Les différentes dimensions de la pauvreté renvoient à des aspects distincts des capacités de l'homme : capacités économiques (revenu, moyens de subsistance, travail décent), humaines (santé, éducation), politiques (moyens d'action, droits, possibilité de se faire entendre sa voix), socioculturelles (statut, dignité) et défensives (insécurité, risque, vulnérabilité). La prise en compte de la problématique homme-femme est essentielle à la réduction de la pauvreté sous tous ses aspects et le maintien de la base de ressources naturelles est nécessaire pour que le recul de la pauvreté soit durable.

Les causes de la pauvreté varient beaucoup d'un pays à l'autre. L'histoire, la géographie et la gouvernance influent sur les modes de développement. Les guerres, les conflits armés et l'effondrement de l'État sont cause de pauvreté et d'aggravation de la pauvreté. La corruption généralisée, la présence d'élites soucieuses de préserver leur rente de situation, le non-respect des droits de l'homme, des administrations inefficaces et le manque de volonté de réformer les politiques et les institutions sont aussi autant d'éléments défavorables à la réduction de la pauvreté. La dégradation de l'environnement, la discrimination à l'encontre des femmes et l'explosion démographique sont d'autres causes importantes de la pauvreté. Sans compter le sida qui est devenu, dans le contexte de la pauvreté, un problème crucial nécessitant une action de grande envergure.

Favoriser une croissance favorable aux pauvres et réduire les inégalités. Une accélération des taux de croissance économique est essentielle (encadré 1) mais ne suffit pas. Les aspects qualitatifs de la croissance - durabilité, composition et équité - sont également importants. Dans de nombreux pays, les inégalités de revenu et d'accès aux actifs compromettent le rythme et la qualité de la croissance, et donc ses effets sur la réduction de la pauvreté. Lorsque les inégalités aboutissent à des conflits et à la violence, elles ont des conséquences désastreuses sur le plan humain mais aussi sur le plan économique. Les organismes de développement doivent par conséquent s'efforcer de renforcer les coalitions favorables à des réformes visant à réduire les inégalités.

Renforcer l'autonomie des pauvres. L'impuissance, l'injustice et l'exclusion perpétuent et accentuent la pauvreté. Les pauvres doivent être en mesure d'exercer leurs droits d'êtres humains et d'influer sur les institutions de l'État et les mécanismes sociaux qui ont des répercussions sur le cours de leur vie. Les approches de la réduction de la pauvreté fondées sur les droits contribuent à renforcer les normes et les institutions qui protègent les droits universels de l'être humain (y compris ceux des enfants et des travailleurs) par l'instauration de systèmes politiques, économiques, juridiques et judiciaires ouverts. Les principales conditions de la démarginalisation des pauvres sont les suivantes :

- Renforcer la participation populaire à la formulation et à la mise en œuvre des politiques ainsi qu'à l'évaluation de leurs effets.

- Favoriser une gestion démocratique, responsable et transparente des affaires publiques.

- Promouvoir les droits de l'homme et les droits des catégories marginalisées.

Accroître les possibilités d'interaction de la société civile et la liberté d'association.
Une même conception

- chez tous les

partenaires au développement - de la pauvreté et de ses multiples dimensions est essentielle pour travailler de concert.

Le rythme et la qualité de la croissance économique sont des aspects fondamentaux de toute stratégie nationale de lutte contre la pauvreté.

Des mesures visant à donner aux pauvres les moyens de faire respecter leurs droits d'êtres humains et de se faire entendre,... 
... à leur faciliter l'accès aux services sociaux de base,...

... à les mettre mieux à même d'assurer la pérennité de leurs moyens de subsistance...

... et à les aider à atténuer leur vulnérabilité et à gérer les risques sont importantes pour faire reculer la pauvreté.
Soutenir la liberté de la presse.

Renforcer l'État de droit et l'administration impartiale de la justice.

Promouvoir des conditions de travail décentes.

- Donner aux pauvres des moyens accrus de peser et d'exercer un contrôle sur la nature, la qualité et les modalités de prestation des services qui leur sont destinés.

Services sociaux de base. La réalisation des objectifs sociaux du développement humain exige des niveaux adéquats de santé, d'éducation, d'approvisionnement en eau, d'assainissement et de protection sociale. Le développement social, capital pour la réduction de la pauvreté, est un droit. Il améliore directement l'existence des hommes et des femmes pauvres, et favorise de manière générale la croissance et le développement. L'éducation, en particulier celle des filles, et les services de santé génésique, sont des éléments essentiels de la lutte contre la pauvreté et contre certains de ses principaux aspects - la maladie, notamment le sida, les risques liés à la maternité et la croissance démographique galopante. La maladie et l'analphabétisme font obstacle au bien-être et à l'emploi productif. Savoir lire et écrire facilite la communication, condition sine qua non de toute participation à la vie sociale et politique. Les dépenses publiques en faveur des services sociaux revêtent une grande importance, si elles sont bien utilisées. Elles doivent être assorties d'incitations et de méthodes de financement favorables aux pauvres, notamment en matière d'assurance sociale, de façon à garantir l'accès et la qualité des services rendus, ainsi que le maintien de leur prix à un niveau abordable. Pour que la situation des pauvres s'améliore, les gouvernements partenaires ont besoin de ressources afin d'investir dans des infrastructures et de fournir des services de base. Pour certains services, en particulier l'approvisionnement en eau et l'assainissement, le paiement de redevances par les utilisateurs ou la participation du secteur privé dans un cadre juridique d'équité sociale peuvent améliorer l'accès, et l'efficacité de la gestion.

Des approches fondées sur la pérennité des moyens d'existence pour répondre aux besoins des populations pauvres en tenant compte de leurs capacités. Ces approches consistent dans un premier temps à interroger les pauvres, hommes et femmes, sur leurs besoins et leurs souhaits, puis à se servir des réponses obtenues pour procéder aux réformes nécessaires dans le contexte du développement durable. Les sources de revenu des pauvres sont très variées et reposent en particulier sur l'utilisation des ressources naturelles, l'artisanat, le commerce et les services. Dans le cadre d'une approche destinée à assurer la pérennité des moyens de subsistance, le développement institutionnel doit viser à rehausser la capacité des pauvres de se sortir de la pauvreté, par exemple grâce à des améliorations continues de la productivité agricole et de la sécurité alimentaire.

Sécurité humaine : réduire la vulnérabilité et gérer les chocs. Pour les pauvres, l'insécurité est une dimension majeure de la pauvreté. Pour assurer la sécurité, il faut prendre des mesures qui protègent les populations des perturbations affectant les nations et les familles. Il faut aussi tenir compte des nombreux risques auxquels sont exposés les pauvres : malnutrition, maladie, chômage, délinquance, vieillesse, violence domestique, conflits armés, catastrophes naturelles et autres risques environnementaux.

\section{Édifier des partenariats efficaces au service de la lutte contre la pauvreté}

La place désormais faite au partenariat en tant qu'instrument de lutte contre la pauvreté impose de repenser complètement les méthodes de coopération pour le développement. Six grands principes doivent régir l'action des organismes de développement : 
L'esprit de partenariat, qui facilite et renforce l'appropriation locale, doit inspirer tous les efforts d'aide au développement.

- L'appropriation nationale des stratégies de réduction de la pauvreté, y compris des politiques et des priorités définies localement, doit être respectée, favorisée et encouragée en permanence dans toutes les interactions avec les partenaires.

- Le soutien apporté aux stratégies nationales de réduction de la pauvreté doit se fonder sur un bilan réaliste des avantages, des inconvénients et des compromis qu'implique l'approche retenue.

- La participation active de différents partenaires et le renforcement de l'autonomie des pauvres sont des éléments essentiels.

- Une meilleure coordination et un engagement durable peuvent rendre les partenariats plus solides et améliorer les résultats obtenus.

- Les actions de développement doivent faire l'objet d'un suivi et d'une évaluation avec les gouvernements partenaires et avec les populations pauvres elles-mêmes, afin de mesurer les performances du partenariat et de s'assurer que les effets produits sont et restent bénéfiques pour les pauvres.

Les partenariats solides et productifs se fondent sur la confiance, la transparence et un même engagement sur les objectifs et les résultats à atteindre. C'est lorsque qu'il se fonde sur des relations réciproques, caractérisées par une définition claire des rôles et des responsabilités de chacun, et sur un dialogue ouvert à tous que le partenariat est le plus efficace. Pour asseoir la confiance mutuelle et l'adhésion à l'action engagée, les partenaires doivent évaluer mutuellement la manière dont ils s'acquittent des responsabilités et des obligations dont ils sont convenus.

L'évaluation des performances des pays partenaires pourrait porter sur les éléments suivants :

- L'ampleur et le rythme des efforts déployés pour orienter leurs stratégies dans un sens favorable aux pauvres en tenant compte des disparités entre hommes et femmes.

- La qualité du dialogue sur les politiques à suivre.

- L'étendue et la qualité des processus locaux de consultation préparatoire à l'élaboration de la stratégie nationale de lutte contre la pauvreté.

- L'impact des politiques et programmes de réduction de la pauvreté.

De même, l'évaluation des performances des organismes de développement pourrait consister à déterminer si :

- Leurs activités de planification et d'exécution vont dans le sens des stratégies déterminées par le pays partenaire et sont coordonnées avec celles des autres partenaires.

Leurs interventions respectent et favorisent l'appropriation locale.

- Les moyens fournis le sont de façon plus souple et prévisible.

- L'aide apportée, y compris sous forme de projets, est intégrée dans le cadre de dépenses du gouvernement partenaire.

- Le soutien consenti a eu un impact en termes de réduction de la pauvreté.
Les organismes

d'aide doivent nouer des partenariats de nature à faciliter et à renforcer

I'appropriation locale des politiques et des activités à l'appui du développement.

\section{Le partenariat} appelle un engagement et une confiance mutuels fondés sur des objectifs communs et des résultats avérés. 
Les organismes d'aide doivent activement appuyer la participation de la société civile au dialogue sur les stratégies de développement et les options qui s' offrent en la matière.
Mobiliser tous les partenaires potentiels au développement afin d'assurer l'appropriation, la durabilité et l'efficacité des actions de développement. La société civile, le secteur privé, le parlement, les collectivités locales, les syndicats, les pauvres eux-mêmes, les organismes extérieurs, toutes ces entités doivent participer à la conception et à la mise en œuvre des stratégies de lutte contre la pauvreté. Cette diversité des acteurs exige une meilleure communication, renforcée par une étroite collaboration, et une bonne connaissance des atouts respectifs et des avantages comparatifs des différents partenaires. Les organismes de développement peuvent jouer un rôle important dans le renforcement de la capacité de la société civile de collaborer avec les pouvoirs publics et dans l'amélioration des mécanismes de consultation. Il faut prendre soin de laisser aux organisations non gouvernementales (ONG), aux chambres de commerce et aux entreprises la possibilité de lancer des initiatives efficaces et novatrices de lutte contre la pauvreté.

Le partenariat implique un dialogue qui ne se limite pas aux instances gouvernementales. Il importe d'élargir à des partenaires plus diversifiés le dialogue qui accompagne la mise au point des stratégies de lutte contre la pauvreté. Il faudra consentir des efforts supplémentaires pour assurer d'emblée, qu'une véritable participation ait lieu dans le cadre de ces consultations pour influencer les choix politiques. Il faut pour cela promouvoir les structures démocratiques à l'échelon local et rechercher dans la société civile des acteurs qui peuvent légitimement parler au nom des pauvres et leur rendre compte de l'évolution de la situation. Il faut cependant veiller à ne pas porter atteinte à la légitimité des gouvernements partenaires mais, au contraire, à respecter ce qu'ils font pour construire et consolider leurs propres institutions démocratiques.

Affecter les ressources de façon à assurer une réduction effective de la pauvreté. Compte tenu du caractère limité des moyens mis à la disposition de l'aide au développement et de l'importance de réduire la pauvreté, il est essentiel que ces moyens soient utilisés aussi efficacement que possible. Les critères gouvernant la répartition des ressources entre les différents pays doivent tenir compte à la fois du nombre et de la proportion de personnes très pauvres, et de l'efficacité avec laquelle chaque pays partenaire devrait pouvoir utiliser l'aide reçue. Pour optimiser l'impact de la coopération pour le développement en termes de réduction de la pauvreté, il faut :

Se concentrer sur les pays les plus pauvres, tout en continuant d'offrir des financements mesurés et ciblés à d'autres pays en développement où la pauvreté est très répandue.

Mettre l'accent sur les grands pays et les pays de taille moyenne, où se trouve la grande majorité des populations pauvres, même si l'aide par habitant reste beaucoup plus élevée dans les petits pays.

- Tenir compte des facteurs d'efficacité de l'aide tels qu'ils ressortent de l'expérience du CAD, laquelle montre l'importance de la volonté politique de combattre la pauvreté et d'un environnement qui contribue à l'efficacité des politiques et des institutions.

- S'assurer que la stratégie de réduction de la pauvreté du pays partenaire est légitime, appropriée et bien adaptée.

Aider les partenaires en proie à de graves difficultés. L'enveloppe allouée à l'aide est aussi mise au service d'autres objectifs de développement que la réduction de la pauvreté monétaire, comme la prévention des conflits, les droits de l'homme, la démocratie participative, l'égalité homme-femme et le développement durable. Souvent, il arrive aussi que des pays aient un besoin aigu de soutien pour s'adapter à des chocs extérieurs, par exemple un afflux de réfugiés fuyant un conflit dans un pays voisin, une catastrophe naturelle, ou une détérioration brutale des termes de l'échange - autant d'événements qui affectent le développement économique et social. 
Collaborer étroitement avec d'autres partenaires extérieurs en cas de situations problématiques. Il faut aider les pays dont les politiques de développement et les institutions sont inadéquates, afin de créer des conditions favorables à l'amélioration de leurs performances. Travailler en partenariat avec de tels pays de façon à susciter l'appropriation locale tout en veillant à l'efficacité de l'aide et à ses retombées sur la pauvreté risque de poser des problèmes. Que se passe-t-il lorsqu'un gouvernement partenaire ne respecte pas les intentions qu'il a affichées ou les engagements qu'il a pris, ou ne les respecte qu'en partie? L'imposition de conditions concernant les mesures à prendre - souvent couplée à un soutien financier et technique - aide parfois les gouvernements (habituellement nouveaux) désireux de procéder à des réformes à progresser dans ce sens. Cependant, les conditions imposées de l'extérieur ne sont généralement ni efficaces, ni durables, ni propres à susciter une appropriation par le pays partenaire, et ont très peu de chances de donner des résultats dans les pays où n'existent pas les bases nécessaires à la mise en place de partenariats. Les bonnes pratiques suivantes semblent se dégager de l'expérience récente :

- La part de l'aide réservée à ces pays doit rester modeste.

- Les partenaires extérieurs doivent partager une approche commune à l'égard du pays partenaire et coordonner leurs efforts de coopération pour le développement ainsi que les actions et mesures qu'ils prennent dans d'autres domaines.

- L'aide apportée doit servir à soutenir les institutions, nationales ou locales, viables et les organisations de la société civile, en mettant l'accent sur l'élimination des obstacles à leur bon fonctionnement : il faut par exemple s'appliquer à relancer le dialogue sur les politiques à suivre, à soutenir les coalitions locales favorables à des réformes et à renforcer les capacités locales de recherche et de concertation sociale.

- La coopération pour le développement peut aussi viser à aider les collectivités locales et les ONG à faire reculer la pauvreté dans des populations bien ciblées, afin de réduire leur vulnérabilité et de garantir la satisfaction de leurs besoins essentiels.

La réduction de la pauvreté s'inscrit dans un processus politique. Les efforts des pays partenaires pour réformer leurs structures et leurs politiques dans un sens favorable à la population pauvre suscitent souvent des difficultés d'ordre politique. Certains groupes s'accrochent à leur pouvoir, à leurs privilèges et à leurs rentes de situation, tandis que d'autres sont plus ouverts à une réforme et à des mesures en faveur des pauvres. Il est compréhensible que les organismes de développement hésitent à intervenir dans des débats politiques internes sur des sujets sensibles, mais ils ne peuvent ignorer ces tensions. En soutenant les efforts que déploie le gouvernement pour engager le dialogue avec la société sur les options et les choix qui s'offrent en matière de développement, ils pourront se familiariser avec la dynamique sociale et politique locale et constituer des alliances stratégiques et des partenariats avec les groupes et les institutions qui sont ouverts aux réformes. Encourager une démocratie pluraliste et participative où les pauvres ont la possibilité de s'exprimer peut aussi être utile à cet égard.

La coordination de l'aide est une responsabilité commune de l'ensemble des partenaires, même si elle doit être instaurée et conduite par les gouvernements partenaires. Les organismes extérieurs doivent s'astreindre à plus de discipline et de persévérance dans les efforts qu'ils déploient pour œuvrer ensemble à la cohérence des approches retenues et au recentrage de l'action engagée sur les besoins fondamentaux et les possibilités de collaboration. Ils doivent mieux partager les informations de façon à permettre une véritable coordination et une utilisation optimale de leur propre cadre d'action par d'autres partenaires, locaux et extérieurs. La difficulté pour la communauté du
Une étroite

coordination entre les

Membres et une

assistance ciblée sont

primordiales lorsque

les résultats obtenus

par un pays

partenaire dans la

voie de la réduction

de la pauvreté ne

sont pas à la hauteur

des engagements

qu'il a pris.
Le resserrement de la collaboration entre les organismes d'aide ne doit pas nuire à l'appropriation locale, ni imposer une charge excessive aux autorités locales. 


\section{Encadré 2. Programme d'action pour les organismes bilatéraux}

Les Lignes directrices définissent comme suit les priorités de l'action que les organismes bilatéraux doivent mener en collaboration avec leurs partenaires pour faire reculer la pauvreté :

Soutenir des stratégies de lutte contre la pauvreté qui soient prises en charge et pilotées par les pays concernés, et se fonder sur les besoins et priorités recensés dans ces stratégies pour la programmation de leurs activités.

Affecter une part plus importante de l'aide au développement aux pays où s'offrent les meilleurs chances de faire reculer la pauvreté compte tenu du nombre de personnes vivant en dessous du seuil de pauvreté absolue, de la détermination des pouvoirs publics à combattre la pauvreté et des résultats constatés de l'action menée. Réserver des ressources pour les pays devant faire face à des chocs extérieurs ou à des situations de conflit, et aux pays aux politiques de développement très insuffisantes.

Réduire la charge que la coopération pour le développement impose aux partenaires locaux en regroupant les efforts (missions conjointes, recherche en collaboration, diagnostics communs, partage des coûts, etc.), en allégeant les contraintes administratives (par exemple en simplifiant, rationalisant et harmonisant les formalités et procédures ; en acceptant, chaque fois que c'est possible, les modèles de documents et de stratégies établis par le partenaire) et en coordonnant leurs approches et leurs actions.

Consacrer le temps et les moyens nécessaires à l'édification, sur une base de réciprocité, de véritables partenariats au service de la lutte contre la pauvreté.
Adapter leurs structures et leurs méthodes de travail aux enjeux et aux besoins des partenariats dans le domaine de la lutte contre la pauvreté (par exemple accroître la présence sur le terrain ; assouplir la prise de décision ; développer les aptitudes du personnel en matière d'accompagnement et de recherche de consensus ; renforcer la transparence et l'obligation de rendre des comptes aux autres partenaires).

Euvrer plus activement au développement des capacités humaines et institutionnelles des pays partenaires.

Veiller à la prise en compte de la problématique hommefemme dans l'ensemble des politiques, programmes et instruments.

Intégrer l'objectif du développement durable, notamment les problèmes d'environnement, dans les cadres stratégiques de lutte contre la pauvreté.

Adopter, dans toute la mesure du possible, un échéancier pluriannuel pour la programmation et le financement des activités de lutte contre la pauvreté, afin d'accompagner les plans et budgets pluriannuels du gouvernement partenaire.

Évaluer les résultats de la coopération pour le développement du point de vue de son impact sur la pauvreté, et élaborer les systèmes et méthodes de suivi et d'évaluation requis.

Favoriser et appuyer les efforts locaux de suivi des programmes de lutte contre la pauvreté et de surveillance de l'utilisation des ressources extérieures et nationales dégagées par les programmes d'allégement de la dette.

Encourager la définition d'indicateurs et d'objectifs locaux de réduction de la pauvreté, et renforcer les capacités locales en matière de statistique, d'analyse, de suivi et d'évaluation.

développement consiste à trouver des modes de collaboration qui n'entravent pas l'appropriation locale et n'imposent pas de charges supplémentaires au pays partenaire.

Une collaboration plus étroite avec les institutions multilatérales et régionales est essentielle. Une collaboration plus efficace avec ces organismes appelle, dans la pratique, une action dans quatre domaines : premièrement, prendre dès le départ et entretenir constamment des contacts avec les personnes compétentes au sein des organismes multilatéraux ; deuxièmement, déterminer d'un commun accord les rôles, les responsabilités et les obligations respectifs des différents partenaires extérieurs dans les processus d'établissement des stratégies nationales de lutte contre la pauvreté ; troisièmement, veiller à ce que les réunions périodiques de coordination restent informelles, fonctionnelles et axées sur les résultats ; quatrièmement, rationaliser et simplifier autant que possible les modalités de financement et de décaissement.

\section{Cadres et instruments de programmation par pays}

Pour que leurs objectifs de réduction de la pauvreté s'inscrivent dans des programmes plus efficaces, les organismes de développement doivent utiliser des cadres stratégiques 
établis par les pays partenaires, une association judicieuse d'instruments d'aide et de bonnes pratiques éprouvées.

Les programmes des organismes de développement doivent avant tout se fonder sur les cadres de développement des pays partenaires. Sous les diverses formes qu'ils ont prises après adaptation à l'échelle nationale, les cadres de planification recommandés par la communauté internationale (comme les CSLP, les SNDD, les CDI ${ }^{4}$ et les bilans communs de pays prévus dans le Plan-cadre des Nations unies pour l'aide au développement) revêtent une importance stratégique pour l'intégration des objectifs de développement du millénaire dans les politiques et mesures nationales. Cependant, ces cadres qui sont étroitement liés et présentent souvent de nombreux points communs doivent être rationalisés de façon à réduire la charge que fait peser sur les pays partenaires la nécessité de se conformer à des instruments de planification multiples. Dans l'idéal, ces divers cadres devraient se fondre en une stratégie nationale unique et complète de réduction de la pauvreté intégrant des priorités économiques, sociales et environnementales.

Les stratégies nationales de réduction de la pauvreté nouvellement mises en place doivent constituer le point d'ancrage de l'aide ex térieure. Ces stratégies doivent émaner du pays, résulter d'un processus participatif, présenter un caractère intégré et être orientées vers les résultats. Les organismes de développement doivent accepter qu'elles se présentent différemment et correspondent à des normes diverses. Ils doivent être conscients des contraintes de capacités dont souffrent souvent les pays, s'y adapter, et prévoir du temps pour une appropriation locale progressive. Ils doivent fixer des objectifs réalistes, en tenant compte de la capacité locale d'exécution des stratégies et du fait qu'une réduction durable de la pauvreté est une entreprise de longue haleine.

Les organismes d'aide doivent axer davantage les programmes par pays sur la lutte contre la pauvreté et accroître leur impact dans ce domaine. Dans la mesure où les pays partenaires sont de plus en plus nombreux à se doter de stratégies de lutte contre la pauvreté, les programmes par pays des organismes d'aide doivent être axés sur les objectifs de réduction de la pauvreté définis par le pays partenaire. Les organismes d'aide peuvent jouer un rôle essentiel dans l'amélioration des processus de formulation des politiques locales en partageant avec les partenaires locaux des informations et les résultats d'analyses, d'études de diagnostic et d'enquêtes auprès des utilisateurs, ainsi que des données et d'autres sources de connaissances. Les programmes doivent exploiter les meilleures connaissances disponibles sur la pauvreté dans le pays. Ils doivent aussi tenir compte des activités des autres organismes d'aide dans le pays afin de refléter l'avantage comparatif de l'organisme concerné.

Les programmes par pays doivent faire appel à divers instruments d'aide en tirant parti des synergies existantes : L'aide peut notamment passer par un soutien financier à des programmes nationaux, régionaux et sectoriels et à des projets. Ce soutien est souvent associé à un dialogue sur les politiques à suivre, et étayé par des activités de coopération technique, le plus souvent intégrées au projet ou programme lui-même. Chaque instrument a ses avantages et ses inconvénients selon le pays partenaire. Dans un souci d'appropriation et de viabilité, la communauté du développement doit toutefois s'orienter vers des modes de coopération axés sur l'appui aux programmes définis par les partenaires plutôt que vers des activités émanant des organismes d'aide. Il faut pour cela mettre l'accent sur l'aide-programme et l'aide sectorielle, mais il est aussi possible d'apporter beaucoup au moyen de projets dès lors que tous ces éléments sont coordonnés de façon que les pays partenaires en prennent la direction. Le choix des instruments et l'équilibre à établir entre actions indirectes et actions ciblées doivent découler d'une analyse des besoins de chaque pays réalisée dans le cadre d'un dialogue avec le gouvernement et les autres parties prenantes.
L'aide mise au service de la lutte contre la pauvreté par les organismes de développement doit se fonder sur la stratégie nationale de développement arrêtée par le pays partenaire.

Le recours à des instruments d'aide variés programmes, projets et aide sectorielle favorise les synergies et les complémentarités. 
L'aide-programme ouvre la voie d'un dialogue permanent sur les politiques favorables à la réduction de la pauvreté. L'aide-programme permet de réduire au minimum les coûts de transaction associés au versement de ressources financières. En donnant au pays partenaire la pleine responsabilité des décisions financières et de la gestion, ce type de soutien répond aux principes de partenariat et d'appropriation locale. Lorsque le contexte politique, économique et institutionnel est propice, l'aide-programme fournie à l'appui d'une stratégie rationnelle de lutte contre la pauvreté aura probablement un impact optimal. Compte tenu du caractère fongible des ressources, les organismes de développement doivent cependant veiller à ce que l'aide-programme qu'ils apportent vienne appuyer un programme de réforme rationnel défini en commun et faisant l'objet d'un suivi. L'allégement de la dette, qui constitue de fait une forme d'aide-programme, repose sur des principes similaires.

L'aide sectorielle peut contribuer à renforcer l'attention portée à la pauvreté et aux inégalités. Les programmes qui s'inscrivent dans une approche sectorielle, peuvent améliorer l'appropriation locale, renforcer le partenariat et créer un contexte institutionnel propice au recul de la pauvreté. Pour exploiter les avantages des approches sectorielles qui se mettent en place au service de la réduction de la pauvreté, les organismes d'aide doivent répondre à de multiples défis. Ils doivent accepter les stratégies sectorielles locales, et promouvoir un dialogue plus ciblé sur l'équité dans le développement social, en particulier dans les secteurs, cruciaux, de la santé et de l'éducation. Ils doivent faire des droits des pauvres et des questions d'égalité homme-femme des préoccupations de première importance dans certains secteurs. Ils doivent donner la priorité au renforcement des capacités locales de formulation des politiques et de mise en œuvre des programmes. Ils doivent faire intervenir la société civile et favoriser les partenariats avec le secteur privé. Et ils doivent rationaliser les méthodes comptables et les procédures de notification. L'augmentation de ce type d'aide dépend aussi de la capacité du pays partenaire de se conformer aux normes de comptabilité et de bonne gestion financière s'appliquant à l'utilisation des fonds extérieurs.

Les projets conduits par les partenaires peuvent avoir une incidence durable sur les moyens de subsistance et le bien-être des bénéficiaires. Les projets de lutte contre la pauvreté produisent d'autant plus d'effets qu'ils s'inscrivent dans un cadre de développement plus vaste, par exemple une stratégie nationale de réduction de la pauvreté ou un programme sectoriel. Ils ont plus de chances de contribuer durablement à la réduction de la pauvreté lorsqu'ils favorisent l'appropriation et la participation, se fondent sur des connaissances locales et sont axés sur l'accroissement des capacités. Les projets doivent être compatibles avec le contexte institutionnel et culturel dans lequel ils s'inscrivent, et recueillir l'adhésion de l'administration centrale, des autorités locales et de la société civile. Les organismes d'aide doivent éviter les petits projets ne touchant qu'une population limitée, ceux-ci faisant peser une charge excessive sur les maigres capacités du pays partenaire. Il faut aussi qu'ils abandonnent les méthodes interventionnistes de micro-gestion, dans la conception comme dans la mise en œuvre, car elles ne permettent pas d'assurer la durabilité des résultats obtenus lorsque le financement extérieur vient à expiration.

Le renforcement des capacités des partenaires de lutter contre la pauvreté doit être un objectif prioritaire de la coopération technique. La coopération technique au service du renforcement des capacités, qu'elle soit autonome ou intégrée dans des projets ou des approches sectorielles, peut jouer un rôle précieux et durable. Pour accroître ses effets, les organismes de développement doivent appliquer de bonnes pratiques ayant fait leurs preuves. Premièrement, il faut faire de l'autonomie et du principe de l'intervention minimale des objectifs stratégiques au lieu de se servir de la coopération technique pour assurer la réalisation de certaines tâches ou combler des lacunes dans les compétences locales. 
Deuxièmement, il faut planifier les activités de coopération technique dans le cadre des stratégies de réduction de la pauvreté et des programmes sectoriels nationaux, plutôt que de se fonder sur des propositions isolées émanant des donneurs. Troisièmement, il faut définir les objectifs en termes de résultats à atteindre plutôt que de ressources à fournir. Quatrièmement, il faut tirer parti des compétences des experts locaux et faciliter, si besoin est, la transmission du savoir-faire par des experts internationaux. Cinquièmement, comme dans le cas des projets et d'une grande partie de l'aide sectorielle, il faut renforcer les institutions et capacités existantes, tant publiques que privées, y compris les universités, au lieu de promouvoir des structures parallèles. Sixièmement enfin, il faut s'assurer que les coûts récurrents resteront supportables et seront pris en compte dans les budgets nationaux ou locaux. Ces bonnes pratiques s'appliquent également à la coopération financière.

Enfin, d'autres instruments qui occupent une place grandissante dans les portefeuilles des organismes de développement, comme l'allégement de la dette, l'aide humanitaire et l'appui à la coopération régionale, peuvent jouer un rôle capital dans la lutte contre la pauvreté.

\section{La cohérence des politiques est importante}

La réduction de la pauvreté exige des politiques cohérentes non seulement en matière de coopération pour le développement. mais aussi entre des politiques des pays Membres de l'OCDE, afin d'éviter que les efforts de lutte contre la pauvreté déployés par les Membres ne soient compromis par les politiques et les interventions d'autres secteurs du gouvernement. La cohérence des politiques au service de la réduction de la pauvreté à l'échelle mondiale doit devenir le fil directeur de l'action menée par chaque Membre au plan national. Les politiques suivies dans des domaines autres que la coopération pour le développement peuvent revêtir une plus grande importance pour le recul de la pauvreté dans les pays en développement. Ainsi, on estime que les droits de douane et subventions s'appliquant aux produits agricoles et manufacturés dans les pays industrialisés se traduisent, pour les pays en développement, par un manque à gagner annuel équivalent au montant annuel de l'aide publique au développement. Si l'on y ajoute l'effet des barrières non tarifaires et de la réglementation des services liés au commerce et des droits de propriété intellectuelle, le chiffre correspondant peut même être trois fois plus élevé.

La cohérence des politiques constitue un défi politique de grande ampleur qui fait ressortir les intérêts de certains groupes particuliers et de la nation dans son ensemble. Les gouvernements poursuivent des objectifs très divers. Il se pourrait néanmoins que les responsables politiques soient aujourd'hui plus nombreux à accorder à la pauvreté une plus grande attention et un degré de priorité plus élevé qu'à d'autres objectifs nationaux. En effet, la pauvreté est source de dysfonctionnements et de désordres dans le monde, et elle a des retombées négatives telles que l'instabilité politique, la dégradation de l'environnement, les migrations illicites, la propagation des épidémies et d'autres problèmes internationaux.

Pour renforcer la cohérence des politiques, il est essentiel que les plus hautes autorités politiques fassent partager à l'ensemble des instances gouvernementales leur volonté de lutter contre la pauvreté à l'échelle mondiale et prennent des mesures afin d'améliorer la coordination des politiques. Presque tous les Membres du CAD ont exprimé publiquement avec force leur détermination à faire reculer la pauvreté. Le défi consiste à faire partager cette détermination à tous les échelons de l'administration et à s'en servir comme point de référence pour la formulation et la mise en œuvre des politiques. Les domaines les plus importants à cet égard sont les échanges et les investissements
Les politiques suivies par les gouvernements Membres de l'OCDE organismes de développement doivent être cohérentes avec l'objectif de réduction de la pauvreté dans le monde. 
En 2000, le Conseil de l'OCDE réuni au niveau des ministres et le CAD réuni à haut niveau ont décidé d'établir une liste des conditions essentielles à la cohérence des politiques qui pourrait servir de référence pour l'élaboration des politiques publiques des pays Membres. Une telle liste est incluse dans les présentes Lignes directrices. Elle couvre tout un éventail de domaines ayant des retombées sur le développement. Elle se veut indicative plutôt qu'impérative et vise à encourager les gouvernements Membres à intégrer systématiquement les questions de développement et de pauvreté dans toutes leurs décisions. Cette liste a été élaborée à la suite de consultations entre experts spécialisés, au sein de l'OCDE, dans les divers domaines d'intervention des pouvoirs publics. Elle doit constituer un petit document distinct que pourront utiliser les différentes sphères de l'administration des pays Membres, et elle servira aussi à faciliter et guider les travaux ultérieurs de l'OCDE sur les questions de cohérence des politiques.
Les domaines dans

lesquels il est

particulièrement

important de veiller à

la cohérence des

politiques dans

l'optique de la

réduction de la

pauvreté concernent, entre autres, les

échanges,

l'agriculture, la

sécurité alimentaire,

la prévention des

conflits et les

questions sociales.

\section{Le partenariat}

appelle des

changements

importants... internationaux, l'agriculture et la sécurité alimentaire, les ressources naturelles et l'environnement, les questions sociales, la gouvernance et la prévention des conflits.

Rendre cohérentes les politiques suivies par les différentes instances gouvernementales est un processus complexe, mais de grands progrès dans cette voie peuvent être réalisés par certains moyens. On peut, par exemple, créer une structure politique, notamment un groupe de travail interinstitutions, pour faciliter les échanges et les consultations au sein des ministères et entre ces derniers, élaborer un projet de lutte contre la pauvreté à l'échelle de l'ensemble du gouvernement, vérifier systématiquement la compatibilité des textes législatifs avec l'objectif de réduction de la pauvreté, ou établir des groupes de réflexion interministériels sur des questions émergentes telles que la prévention des conflits.

Les organismes de développement ont un rôle important à jouer en tant que défenseurs des objectifs du développement et doivent veiller à ce que des mécanismes soient prévus pour assurer concrètement la cohérence des politiques. Ces mécanismes doivent bien entendu s'appliquer aux objectifs de développement et aux autres objectifs nationaux. Des réunions officielles sont un outil précieux pour l'amélioration de la cohérence, mais il est essentiel également d'acquérir une culture des contacts informels.

\section{Changer notre manière de penser et d'agir}

Les organismes d'aide devraient réfléchir à la manière dont ils vont aborder les changements institutionnels qui s'imposeront si la relation classique donneur/bénéficiaire est appelée à être redéfinie et transformée en une relation fondée sur le principe de partenariat, régie par les priorités des autorités du pays en développement et facilitée par un partage des connaissances et l'instauration d'un dialogue sur les politiques à suivre. L'évolution qui se dessine appellera dans bien des cas des changements dans les modes d'organisation, les pratiques, les systèmes d'incitation et la culture interne des organismes d'aide. Il s'agira notamment, si l'on veut vraiment progresser, de répondre aux questions suivantes :

\section{Que faut-il faire pour intégrer de façon systématique la réduction de la pauvreté dans toutes les activités des organismes d'aide?}

Faire naître une véritable détermination de la hiérarchie, tant au niveau politique qu'au niveau de la définition de l'action, de façon à mobiliser et focaliser la motivation et la participation de l'ensemble du personnel, des autres instances publiques et de la société civile autour d'une action plus résolument et plus 
fermement tournée vers le soutien à la lutte contre la pauvreté dans les pays partenaires. Il faut aussi que les organismes d'aide se donnent une ambition claire, des orientations et une stratégie pour aider les partenaires à faire reculer la pauvreté, en s'appuyant notamment sur des stratégies par pays, des approches sectorielles et des activités menées dans le cadre de projets. L'objectif de réduction de la pauvreté doit imprégner l'ensemble des processus de planification et servir de critère pour la sélection et l'approbation des programmes et projets.

- Compte tenu du caractère pluridimensionnel de la démarche à suivre, reconnaître la nécessité de revoir les modes d'organisation afin de faciliter la coordination, les synergies en termes de compétences et les échanges de savoir au sein des organismes d'aide. Les bonnes pratiques en la matière conjuguent deux démarches : l'une visant à rendre l'ensemble du personnel responsable de l'action menée pour faire reculer la pauvreté, l'autre consistant à désigner des « relais » ou « chefs de file » chargés d'amorcer le processus et d'enclencher la dynamique de changement institutionnel et d'apprentissage. Ces relais auront besoin de ressources et d'autorité pour agir efficacement.

- Admettre la nécessité pour les organismes d'aide de faire appel à des spécialistes offrant une palette de compétences plus diversifiée (et capables en particulier de veiller à l'intégration de questions transversales importantes comme l'égalité homme-femme, la gestion des affaires publiques, l'environnement et les approches participatives). Il faudra doter les bureaux locaux de spécialistes de la pauvreté (notamment des spécialistes en macro-économie et des experts techniques). On pourra recourir à des personnels déjà présents sur place ou dans des centres régionaux, auxquels des agents du siège de l'organisme d'aide apporteront leur concours. Des programmes de formation aux questions et aux méthodes de lutte contre la pauvreté devront être mis sur pied afin de développer la capacité du personnel de réagir avec clairvoyance et en faisant preuve de recul.

Encourager le travail en équipe en supprimant le cloisonnement entre les spécialités de façon à mieux faire face au caractère pluridimensionnel de la pauvreté et à dépasser le stade des approches strictement sectorielles ou des stratégies conduites par les donneurs, ce qui aura des incidences sur la manière dont les organismes d'aide déploient et gèrent leurs ressources en personnel.

Comment faire que les organismes d'aide collaborent plus efficacement sur le terrain avec leurs divers partenaires, sous la conduite des autorités locales?

- Alléger les contraintes créées par la multiplicité des exigences administratives et financières et améliorer la coordination interne des politiques et des activités. L'effort devra porter avant tout sur la rationalisation, la simplification et l'harmonisation des pratiques, des procédures et des obligations en matière de notification, dans le respect des exigences de transparence de chaque organisme d'aide. Un soutien au renforcement des capacités locales en matière de reddition des comptes et de transparence de l'utilisation de l'aide (gestion financière, comptabilité et suivi) permettra d'améliorer la confiance des organismes d'aide et de faciliter toutes les actions ayant pour but de faire concorder les systèmes et procédures des organismes d'aide avec ceux des partenaires des pays en développement. Ce renforcement des capacités ne doit pas donner lieu à des activités indépendantes de chaque organisme, mais résulter d'une aide coordonnée.
... dans les modes d'administration et de gestion des organismes d'aide...

... ainsi que dans leurs systèmes, leurs structures, leurs pratiques et leurs cultures internes. 
Une simplification

des exigences des

organismes d' aide en

matière de

notification et de

reddition de comptes

et une

décentralisation vers

le terrain des

ressources en

personnel et de la

prise des décisions

faciliteront les

relations de

partenariat.
La formation, les

méthodes de

recrutement et des

systèmes appropriés

de récompense et

d'incitation

contribuent à

inculquer au

personnel des

réflexes et des

comportements

conformes aux

exigences du

partenariat.
Améliorer les moyens qu'ont les organismes de développement d'aider les gouvernements partenaires à élaborer leurs propres stratégies de réduction de la pauvreté et à collaborer plus efficacement avec les autres partenaires et parties prenantes. Cela pourra nécessiter un changement d'attitude de la part des organismes d'aide quant au rôle de la société civile dans la formulation des politiques. Les Membres doivent se montrer réalistes quant au temps nécessaire pour susciter une large adhésion aux stratégies de lutte contre la pauvreté et pour mobiliser les ressources requises du côté des pouvoirs publics des pays partenaires, en particulier lorsque ceux-ci ne disposent que de faibles capacités.

Décentraliser des ressources humaines et des pouvoirs de décision au profit des représentations locales. Cela peut en effet se révéler utile à plusieurs titres. D'une part, cela permet de mieux appréhender la pauvreté dans le contexte du pays et de mieux réagir à l'évolution des conditions locales. D'autre part, cela facilite la constitution d'équipes pluridisciplinaires et un resserrement du dialogue et du partenariat grâce à l'instauration de contacts étroits et permanents avec d'autres partenaires locaux.

Dans les décisions de décentralisation, mettre en balance les avantages ci-dessus qui en découlent avec les éventuels inconvénients : augmentation des coûts et surexploitation ou dispersion excessive des compétences techniques. Par ailleurs, il convient de noter que la décentralisation n'est pas nécessairement une solution réaliste et/ou idoine dans le cas d'organismes d'aide de petite taille, compte tenu des surcoûts qui en résultent et du surcroît d'efforts qu'elle nécessite pour assurer la qualité des programmes définis localement et de la reddition des comptes. Cette remarque plaide encore plus fortement en faveur d'une intensification de la collaboration et des échanges de savoir-faire et d'informations entre tous les organismes d'aide, ainsi que d'une mobilisation accrue des compétences locales.

Comment rehausser la capacité des membres du personnel des organismes d'aide de travailler en partenariat et leur motivation à le faire ?

Inviter le personnel d'encadrement à mettre l'accent sur l'amélioration des compétences de nature à favoriser le partenariat (sens de la concertation, de la diplomatie et de la négociation, expérience de la coordination) et ouvrir de nouvelles possibilités d'échanges de personnel, de perfectionnement et de travail en équipe.

Instituer des procédures de recrutement et des systèmes d'incitation (à l'intention du personnel permanent, temporaire et diplomatique) privilégiant les compétences et les résultats dans le(s) domaine(s) de la lutte contre la pauvreté et de l'instauration d'une croissance favorable aux pauvres, la capacité de travailler en équipe et les efforts déployés pour enclencher et alimenter une dynamique de coordination avec les autres membres du personnel et les partenaires.

Comment faire que les modes d'organisation et les procédures des organismes d'aide favorisent une plus grande cohérence des politiques à l'échelle de l'ensemble de l'administration des pays Membres ?

Mettre en place des dispositifs ou améliorer les dispositifs existants pour résoudre les conflits d'intérêts ou concilier des stratégies aux effets contradictoires. L'amélioration de la cohérence des politiques exige en outre une volonté de consacrer du temps et des ressources à la mise en évidence et à l'analyse des problèmes, et à la réflexion collective dans le cadre de diverses enceintes nationales et internationales. Plus précisément, il est capital, si l'on veut améliorer la cohérence globale des efforts de coopération pour le développement, de rapprocher 
et de rendre plus cohérentes les positions des organismes d'aide et des autres instances gouvernementales concernant les relations avec les institutions multilatérales.

\section{Atteindre les objectifs que nous nous sommes fixés}

La réduction de la pauvreté dans les pays en développement passera assurément par un recentrage des activités de coopération pour le développement et un renforcement de la cohérence des politiques. Pourtant, les efforts entrepris en ce sens n'auront que peu d'effet sans la mobilisation, le concours et la valorisation des ressources d'autres partenaires, notamment des gouvernements des pays partenaires, de la société civile et du secteur privé, mais surtout des pauvres eux-mêmes. Pour atteindre les objectifs que nous nous sommes fixés, il nous faudra absolument unir toutes ces forces. Ces Lignes directrices proposent des informations, des données d'expérience et des orientations qui pourront aider les organismes d'aide bilatérale à instaurer, avec les pays partenaires, entre eux et avec les organismes multilatéraux, une collaboration de plus en plus fondée sur la coordination et la collégialité des décisions. Tous les partenaires au développement doivent s'appliquer de leur mieux à faire en sorte que les progrès qui marqueront ce siècle dans le domaine du développement résultent d'un véritable esprit de partenariat et bénéficient à tous sans exception. 


\section{Notes}

1. Selon la norme internationale fixée à $\$ 1$ (aux parités de pouvoir d'achat - PPA) par jour. Si l'on utilise la norme de \$ 2 par jour, le nombre d'individus en situation d'extrême pauvreté passe à 2.8 milliards, soit $60 \%$ de la population des pays en développement.

2. Les pays en développement sont ici désignés par les expressions «pays partenaires »ou « gouvernements partenaires », et non « bénéficiaires », pour bien souligner l'importance qui est attachée aux mécanismes et aux modalités de partenariat. De même, la communauté de l'aide bilatérale est désignée par « les organismes de développement» ou les « organismes d'aide » (et non par les «donneurs »). L'expression «parties prenantes » renvoie, pour sa part, à tous ceux qu'intéresse ou que préoccupe la réduction de la pauvreté.

3. La présentation qui en est donnée ici doit encore être approuvée par l'Assemblée générale des Nations unies en 2001 dans le cadre de son examen des rapports qui doivent lui être soumis chaque année dans le prolongement de la Déclaration du millénaire.

4. CDI : Cadre de développement intégré de la Banque mondiale ; CSLP : Cadre stratégique de lutte contre la pauvreté ; SNDD : Stratégie nationale de développement durable. 


\section{Introduction générale}

\section{La pauvreté dans les pays en développement}

Ces trente dernières années, des progrès considérables ont été accomplis sur le plan économique et social dans l'ensemble des pays en développement. L'espérance de vie s'y est allongée de plus de 20 ans (passant de 41 à 62 ans). Le taux de mortalité infantile a diminué de moitié et le taux de scolarisation dans l'enseignement primaire a été multiplié par deux. La proportion des familles habitant en milieu rural qui ont accès à l'eau propre a été portée de $10 \%$ à plus de $70 \%$. En ce qui concerne les revenus moyens, la santé et l'alphabétisation, l'amélioration de la situation a été plus sensible au cours de ces trente dernières années dans les pays en développement qu'en cent ans dans les pays de l'OCDE.

La pauvreté monétaire n'en demeure pas moins un problème de taille. Bien que le pourcentage de la population totale des pays en développement et des pays en transition vivant dans une extrême pauvreté ait légèrement reculé depuis une dizaine d'années, passant de $28 \%$ à $24 \%$, le nombre de pauvres n'a cessé de croître dans la plupart des régions (à l'exception de l'Asie de l'Est). La population vivant dans une extrême pauvreté reste fortement concentrée dans certaines zones : c'est en Asie du Sud que l'on compte le plus grand nombre de pauvres ( $43 \%$ du nombre total de pauvres dans le monde) et en Afrique que la proportion de la population vivant dans la pauvreté est la plus élevée $(48 \%)$.

L'extrême pauvreté est un fléau qui frappe aujourd'hui encore une personne sur quatre (soit 1.2 milliard d'individus) dans le monde en développement ${ }^{1}$. La pauvreté demeure omniprésente, implacable et inacceptable. En l'espace de dix ans seulement, le nombre de pauvres en Afrique subsaharienne a augmenté de plus d'un tiers. Dans les pays en développement, un enfant sur trois ne va pas au bout des cinq années d'enseignement primaire qui constituent le minimum requis pour acquérir les connaissances de base. Un enfant sur douze nés cette année mourra de maladie ou de malnutrition avant d'avoir atteint l'âge de cinq ans. L'épidémie de VIH/sida poursuit son inexorable progression, anéantissant des décennies de progrès sur le front du développement et faisant peser un fardeau énorme et tragique sur l'ensemble du monde en développement dont les enfants, les familles, les communautés et les sociétés sont les premières victimes. Des décisions radicales et énergiques doivent absolument être prises pour permettre d'améliorer ces chiffres, a fortiori si l'on considère les difficultés que ne manquera pas de créer l'augmentation du nombre d'habitants au cours des 25 prochaines années, estimée à 2 milliards dans les pays en développement, soit l'équivalent de $97 \%$ de l'accroissement démographique mondial.

Un recul de la pauvreté passe par une croissance vigoureuse, à large assise, et l'amélioration de l'accès aux services sociaux. Il nécessite aussi une réduction des inégalités. Même si, dans les années 90, les pays en développement ont enregistré dans l'ensemble une croissance économique annuelle raisonnable, de $2.6 \%$ par habitant en moyenne, le nombre total de pauvres est resté inchangé. En effet, les inégalités se sont accentuées de manière générale, en particulier entre les pays, mais aussi à l'intérieur des pays, et les inégalités entre les hommes et les femmes constituent un aspect important de ces disparités de plus en plus grandes. Les Nations unies estiment que près de $70 \%$

\author{
Des avancées \\ remarquables dans la \\ voie du \\ développement \\ économique et du \\ bien-être ont été \\ obtenues depuis un \\ demi-siècle dans les \\ pays en \\ développement...
}

... mais la pauvreté demeure omniprésente, implacable et inacceptable.

Une croissance économique vigoureuse, un accès plus facile aux services publics essentiels et une réduction des inégalités - entre les sex es en particulier - sont fondamentaux pour faire reculer la pauvreté... 
... de même qu'un effort soutenu et coordonné, appuyé par des ressources suffisantes, dans tous les compartiments de l'action gouvernementale et les domaines couverts par les activités de coopération pour le développement.

Les Membres du CAD ont regroupé ici, sous une forme condensée, les connaissances et le savoir-faire accumulés afin de renforcer l' efficacité de leur action au service de l'objectif international de réduction de la pauvreté. des personnes les plus pauvres dans le monde sont des femmes et certains indicateurs sociaux témoignent d'une forte discrimination à l'encontre des femmes. Ainsi, l'analphabétisme des femmes jeunes et adultes est près de deux fois plus élevé que celui des hommes et le taux de mortalité des petites filles est supérieur de $28 \%$ à celui des petits garçons. Pour réduire la pauvreté, il faut s'attaquer aux inégalités, car elle cède d'autant plus rapidement devant la croissance économique que les inégalités sont moins marquées ${ }^{2}$.

Les progrès réalisés au cours des décennies précédentes permettent certes d'espérer avec confiance que la pauvreté pourra être vaincue et que le développement deviendra une réalité. Il n'y a toutefois pas lieu de se montrer exagérément optimiste. L'éradication de la pauvreté exigera un effort soutenu et coordonné, appuyé par des apports de ressources suffisants, dans tous les compartiments de l'action gouvernementale et dans tous les domaines couverts par les activités de coopération pour le développement. Elle exigera également la mobilisation de tous les partenaires au développement. Les Lignes directrices du CAD pour la réduction de la pauvreté, vecteurs des connaissances et de l'expérience acquises par la communauté du développement dans le domaine de la lutte contre la pauvreté, ont vocation à éclairer ses décisions et à tracer des orientations pour l'avenir.

\section{Une détermination renouvelée, au sein des instances internationales, à faire reculer la pauvreté}

En 1996, les ministres de la coopération pour le développement des pays de l'OCDE ont adopté un cadre qui ouvre de nouveaux horizons dans le domaine de la coopération pour le développement. La stratégie préconisée par le Comité d'aide au développement de l'OCDE dans le document intitulé Le rôle de la coopération pour le développement à l'aube du XXI siècle est l'expression d'une conception de la coopération pour le développement qui repose sur le principe de partenariats construits autour de stratégies de développement élaborées et conduites par les pouvoirs publics et la société civile des pays en développement. Les Membres du CAD s'y engagent par ailleurs à œuvrer en collaboration avec leurs partenaires à la réalisation d'objectifs internationaux de développement précis, chiffrés et assortis d'échéances. Ces objectifs - renvoyant à nombre des aspects fondamentaux de la pauvreté ont été approuvés par la communauté internationale dans les domaines économique, environnemental et social lors des conférences mondiales organisées dans les années 90 sous les auspices des Nations unies. Les Membres du CAD ont par la suite réaffirmé leur position dans une déclaration rendue publique en mai 2000 sous le titre Le partenariat au service de la lutte contre la pauvreté : des principes à la pratique. Les objectifs internationaux de développement ont été intégrés dans l'ensemble plus large d'objectifs de développement du millénaire - concernant entre autres la faim, l'accès à une eau sûre, le VIH/sida, les habitants de taudis et l'instauration d'un partenariat mondial au service du développement - énoncés en 2000 dans la Déclaration du Sommet du Millénaire des Nations unies. Avancée importante sur la voie de la mise en œuvre de la stratégie pour le $\mathrm{XXI}^{\mathrm{e}}$ siècle, les Membres du CAD ont en outre décidé de regrouper dans les présentes Lignes directrices pour la réduction de la pauvreté, sous une forme condensée, les connaissances et le savoirfaire acquis collectivement dans le domaine de la lutte contre la pauvreté.

Pour l'élaboration de ces Lignes directrices, les Membres du CAD se sont efforcés de mettre en pratique le principe du partenariat en soumettant leur projet de texte à un large éventail de spécialistes de la pauvreté représentant les pouvoirs publics, la société civile et les milieux universitaires dans le cadre d'un processus de consultation qui s'est déroulé en Afrique, en Amérique latine, en Asie et en Europe. Les éléments d'information, 
les préoccupations et les points de vue qui se sont dégagés au cours de ces échanges ont inspiré le contenu des Lignes directrices et, en particulier, les idées et les recommandations qu'elles proposent pour faciliter l'instauration d'un réel partenariat avec d'autres acteurs du développement.

Les Lignes directrices présentent un programme d'action ambitieux dans le domaine du partenariat, qui appelle des changements dans la manière dont les organismes de développement ${ }^{4}$ appréhendent la pauvreté, dont ils planifient et mettent en œuvre l'aide accordée pour lutter contre la pauvreté, dont ils fonctionnent sur le terrain et au niveau du siège, et enfin dans la manière dont ils envisagent le renforcement de leurs capacités internes afin de mieux remplir les missions qui leur incombent. Dans le même temps, parce qu'elles définissent des droits, des responsabilités et des obligations inhérents à la mise en œuvre d'approches fondées sur le partenariat, les Lignes directrices contribuent très utilement à donner une substance au modèle de partenariat pour le développement ébauché dans la stratégie pour le $\mathrm{XXI}^{\mathrm{e}}$ siècle et à en délimiter la portée.

\section{Vue d'ensemble des Lignes directrices du CAD pour la réduction de la pauvreté}

Ces Lignes directrices ont pour objet d'aider les organismes de développement à faire de la lutte contre la pauvreté l'axe central de leurs décisions stratégiques et de leurs activités sur le terrain. Elles précisent des concepts, proposent des définitions, suggèrent des priorités et décrivent des pratiques exemplaires, des programmes, des instruments et des moyens susceptibles d'être utilisés pour faire reculer la pauvreté. Elles ouvrent de nouvelles perspectives dans un certain nombre de domaines, notamment :

- La définition de méthodes et principes communs pour appréhender, mesurer et cibler la pauvreté. Les Lignes directrices commencent par une vue d'ensemble des relations entre les différents principes et méthodes appliqués dans le domaine de la pauvreté et trace le canevas dans le cadre duquel il convient d'appréhender et d'analyser la nature et les causes de la pauvreté. Il s'agit en l'occurrence d'éclairer le lecteur sur le caractère pluridimensionnel de la pauvreté, sur la question de savoir qui sont les pauvres et enfin sur la manière de définir des mesures de lutte contre la pauvreté, de les combiner et d'en suivre la mise en œuvre.

- L'instauration de partenariats au service de la réduction de la pauvreté. La nécessité d'assujettir rigoureusement le soutien accordé par les organismes d'aide aux objectifs de réduction de la pauvreté et de pilotage des stratégies par les gouvernements des pays partenaires constitue un défi majeur pour la communauté du développement. Le chapitre 2 traite de la manière dont les organismes d'aide peuvent coopérer avec d'autres acteurs afin de nouer des partenariats solides dans des situations différentes d'un pays à l'autre et d'appuyer les efforts déployés à l'échelon national pour définir des stratégies valables de lutte contre la pauvreté.

- L'adoption de procédures de planification, de cadres et d'instruments efficaces au niveau national. Le chapitre 3 examine les modalités pratiques du travail en partenariat, en s'intéressant plus particulièrement aux dispositifs existants régissant l'organisation et la mise en œuvre des activités de coopération pour le développement. Il présente toute une série d'outils et de méthodes efficaces, dont il analyse les avantages et les inconvénients respectifs au regard de l'évolution des pratiques et des perspectives de réduction de la pauvreté.
Ces lignes directrices précisent les concepts et les définitions en rapport avec la pauvreté, suggèrent des priorités et décrivent des pratiques exemplaires qui aideront à choisir les politiques, programmes, instruments et canaux d'acheminement les plus propices pour faire reculer la pauvreté. 
Les Membres du CAD $s$ 'engagent, pour lutter contre la pauvreté, à intégrer les considérations économiques, sociales et environnementales et les questions de gouvernance dans une approche globale du développement.
La cohérence entre l'ensemble des politiques menées par les Membres de l'OCDE et l' objectif de réduction de la pauvreté. La cohérence des politiques conduites par les gouvernements des Membres (notamment en matière d'échanges, d'agriculture et d'environnement) est fondamentale pour s'assurer que les efforts entrepris par les Membres pour faire reculer la pauvreté ne seront pas compromis par des mesures ou des décisions prises dans d'autres domaines de l'action gouvernementale. Le chapitre 4 porte sur la cohérence, au niveau national, entre les stratégies en matière d'aide et les autres politiques, ainsi que sur la cohérence, au niveau international, entre les activités de coopération de portée régionale et mondiale.

- Le développement d'une culture institutionnelle du changement et du perfectionnement. La stratégie pour le $\mathrm{XXI}^{\mathrm{e}}$ siècle préconisée par le CAD a suscité chez les organismes de développement un désir de revoir leur manière de gérer leurs activités afin de se conformer aux principes du partenariat, de l'appropriation locale et de la reddition des comptes. Le dernier chapitre des Lignes directrices pour la réduction de la pauvreté porte principalement sur les modes de fonctionnement interne des organismes de développement : il étudie les modifications susceptibles d'être apportées aux modèles d'organisation, aux systèmes de gestion et à la culture institutionnelle en vigueur dans ces organismes afin d'accroître la capacité de ces derniers de contribuer efficacement à la lutte contre la pauvreté.

Les Lignes directrices sont spécifiquement centrées sur les préoccupations et le fonctionnement des instances bilatérales d'aide au développement des pays de l'OCDE. Cependant, les informations concrètes et les idées qu'elles proposent concernant la manière dont les partenaires au développement peuvent œuvrer ensemble à la réduction de la pauvreté ne manqueront pas de se révéler utiles à tous les praticiens intervenant dans le domaine du développement.

\section{Objet et vocation des Lignes directrices}

Les Lignes directrices du CAD pour la réduction de la pauvreté ont pour objet d'aider les organismes bilatéraux d'aide au développement à appuyer les efforts que déploient leurs partenaires des pays en développement pour combattre la pauvreté. Elles sont l'expression d'une unité de vue parmi les Membres du CAD et de leur détermination commune à s'employer encore plus activement à faire reculer la pauvreté, tant par souci de solidarité avec les plus pauvres que par volonté de faire respecter les droits universels de l'être humain.

En adoptant ces Lignes directrices, les Membres résolvent de faire de la réduction durable de la pauvreté le pivot de la coopération pour le développement. Un nombre croissant de Membres du CAD est en effet convaincu que l'objectif suprême de la coopération pour le développement doit être de combattre la pauvreté puis de l'éliminer dans le contexte du développement durable, et que la réalisation de cet objectif nécessite l'intégration des considérations économiques, sociales et environnementales et des questions dans une approche globale du développement à l'échelon de chaque pays. En adoptant ces Lignes directrices, les Membres adhèrent en outre à une vision et à une conception communes de la pauvreté et des stratégies, cadres et priorités à retenir pour la combattre. Leurs efforts y gagneront en cohérence et en complémentarité au sein du CAD et, grâce à la parfaite compatibilité des Lignes directrices avec les cadres analogues en vigueur sur le plan international, avec l'ensemble du système international. 
Les Membres du CAD s'engagent par ailleurs à mettre tout en œuvre pour appliquer dans leurs activités les principes énoncés dans les Lignes directrices, et notamment pour faire des stratégies de réduction de la pauvreté arrêtées par les pays partenaires l'axe central de leurs programmes par pays, pour alléger le fardeau qu'imposent aux pays partenaires leurs procédures et leurs exigences, et enfin pour améliorer la cohérence des politiques au sein de leurs organismes d'aide et administrations respectifs et entre ces derniers. Ils conviennent également de s'attacher, dans la mesure où les conditions et les contraintes institutionnelles le permettront, à suivre les recommandations formulées, notamment en veillant à améliorer la souplesse et la prévisibilité de leur aide, à instaurer des conditions propices à la décentralisation du pouvoir de décision et à utiliser les cadres définis par les pays partenaires en matière de planification, et en s'assurant que les formes de soutien choisies - aide-programmes ou aide-projets - sont adaptées aux besoins et aux contraintes locales en matière de financement des coûts récurrents, de personnel qualifié et d'impact sur l'environnement.

Les Membres du CAD sont conscients de la diversité des atouts et des ressources dont ils disposent pour contribuer à combattre la pauvreté, et de la nécessité de s'adapter avec souplesse et créativité aux besoins et aux priorités définis par les gouvernements et les autres parties prenantes des pays partenaires. Ils s'accordent à reconnaitre qu'il demeure important de pouvoir s'appuyer sur un secteur privé dynamique pour générer des emplois et des revenus, de favoriser un développement durable pour remédier à la dégradation de l'environnement, d'améliorer la gestion des affaires publiques afin d'encourager l'intégration et la participation de tous, et enfin de renforcer les institutions de manière à consolider les capacités et les systèmes d'administration locaux. Si ces aspects plus généraux de la coopération pour le développement sont traités de façon plus approfondie dans d'autres Lignes directrices du CAD, les Membres ne doutent pas de leur contribution potentielle à la réduction de la pauvreté et conviennent de veiller à l'intégration des préoccupations et considérations relatives à la pauvreté dans le processus d'élaboration de leurs stratégies dans ces divers domaines.

L'adoption des Lignes directrices constitue en outre un signal révélateur de la manière dont les acteurs de la coopération pour le développement perçoivent les enjeux qui, à l'échelle mondiale, et plus précisément, dans leurs propres organisations, sont appelés à prendre une importance grandissante dans la perspective de la réduction de la pauvreté. Elle témoigne de leur volonté d'engager des efforts ciblés et résolus afin de rechercher des solutions nouvelles pour faire reculer la pauvreté, de faire face aux déséquilibres et aux besoins créés par la mondialisation, les biens publics mondiaux et la fracture numérique, et de rendre les structures et cultures institutionnelles en place compatibles avec les conditions préalables à l'instauration de partenariats.

\section{Utilisation des Lignes directrices}

Les Lignes directrices présentent des informations pratiques sur la nature de la pauvreté et sur les moyens à mettre en œuvre pour la combattre. Elles intéressent donc le personnel des organismes d'aide bilatérale en poste sur le terrain et au siège. Elles proposent en outre des orientations utiles concernant la formulation de stratégies appropriées et la fixation des priorités, la manière de travailler avec les différents partenaires dans les pays en développement et dans le cadre du système international, et enfin les modifications à opérer dans les modes de direction, d'organisation et d'intervention des organismes d' aide bilatérale afin que ceux-ci soient à même d'établir des partenariats et d'intégrer de façon systématique la lutte contre la pauvreté dans leurs activités. Ces informations ne manqueront pas de se révéler utiles aux instances
Chaque Membre du CAD possède des capacités, des compétences et des ressources qui lui sont propres et qu'il peut mettre au service de la lutte contre la pauvreté. 


\section{Il y a beaucoup à apprendre de l'expérience des autres, y compris des pauvres qu'il faut écouter et dont le point de vue doit être pris en compte.}

dirigeantes des organismes d'aide bilatérale, ainsi qu'aux cadres en poste sur le terrain et aux responsables de la stratégie et des ressources humaines.

Pourtant, compte tenu de la nécessité d'être succinct et de l'étendue du sujet à traiter, les Lignes directrices ne font qu'esquisser des orientations générales. Le CAD se propose donc, afin d'en élargir la portée et d'en enrichir le contenu, d'étudier plus avant certains aspects essentiels des stratégies de lutte contre la pauvreté pour dégager des pratiques exemplaires.

Si des efforts considérables ont été déployés pour faire en sorte que les Lignes directrices pour la réduction de la pauvreté tiennent compte des travaux de recherche, des connaissances et des enseignements de l'expérience les plus récents, celles-ci sont encore appelées à évoluer. À bien des égards en effet, qu'il s'agisse d'encourager l'autonomisation de la population, une meilleure gestion des affaires publiques, la participation ou le renforcement des institutions, les spécialistes du développement sur le terrain commencent à peine à se faire une idée des moyens d'action, et d'interaction, qui contribuent le mieux à faire reculer la pauvreté. En outre, chaque pays partenaire est un cas particulier, et unique, ce qui voue d'avance à l'échec toute tentative pour proposer un modèle universel de lutte contre la pauvreté. Il reste encore beaucoup à apprendre sur les bonnes pratiques à appliquer dans le domaine en constante évolution de la coopération pour le développement, notamment en se mettant à l'écoute des pauvres eux-mêmes afin de tenir compte de leur point de vue. 


\section{Notes}

1. Si l'on retient comme norme internationale un revenu quotidien de $\$ 2$, les chiffres obtenus sont respectivement de six personnes sur dix et 2.8 milliards d'individus.

2. Global Economic Prospects and the Developing Countries, Banque mondiale, 2001, page ix. Martin Ravallion: Growth, Inequality and Poverty: Looking Beyond Averages, Banque mondiale, 2000.

3. Voir la liste des objectifs de développement du millénaire, page 19.

4. La communauté de l'aide bilatérale au développement est ici désignée par les expressions « organismes de développement » ou « organismes d'aide », et non «donneurs », terme qui serait inadéquat dans le contexte des processus et conditions propres au partenariat. De même, les pays en développement sont désignés par les expressions «pays partenaires » ou « gouvernements partenaires » (et non «bénéficiaires »). Quant à l'expression « parties prenantes », elle renvoie à la société civile des pays en développement. 


\section{Notions et approches}

\section{Introduction}

Pour être efficace, la lutte contre la pauvreté nécessite une réflexion stratégique reposant sur des notions et des approches cohérentes et précises. Différentes perceptions de la pauvreté conduisent à différentes manières de s'y attaquer. Partager une même conception, claire, de la pauvreté permet d'élaborer un programme d'action commun avec les partenaires au développement. Ce premier chapitre des Lignes directrices replace les définitions actuelles de la pauvreté et du bien-être dans un cadre conceptuel qui relie l'objectif consistant à faire reculer durablement la pauvreté aux causes fondamentales de ce fléau ainsi qu' aux stratégies et actions qui s'imposent pour le combattre'.

Une approche stratégique de la lutte contre la pauvreté appelle une analyse en cinq phases :

i. Qu' est-ce que la pauvreté ? - Clarifier et définir le concept.

ii. Qui sont les pauvres ? - Déterminer les seuils de pauvreté et les catégories sociales.

iii. Comment mesurer la pauvreté et suivre son évolution ? - Choisir les méthodes et les indicateurs.

iv. Comment expliquer la persistance de la pauvreté ? - Analyser les causes structurelles et dynamiques.

v. Quelles sont les mesures à prendre ? - Formuler des stratégies et des programmes.

Les organismes de développement doivent réfléchir à toutes ces questions, et y trouver des réponses dictées par l'expérience et l'observation, en ayant soin de le faire en partenariat avec d'autres instances qui œuvrent en faveur de la réduction de la pauvreté : services gouvernementaux à tous les niveaux, autres organismes bilatéraux ou multilatéraux et organisations de la société civile.

\section{Qu' est-ce que la pauvreté ?}

\section{La pauvreté, une notion de plus en plus vaste}

La notion de pauvreté recouvre différentes formes de privation. D'une manière générale, elle renvoie à l'incapacité d'atteindre des normes de bien-être sur le plan économique et social, mais aussi sur d'autres plans. Le caractère pluridimensionnel de la pauvreté est désormais largement reconnu. Il est solidement attesté par des travaux de recherche, parmi lesquels d'importantes études participatives visant à montrer ce que les pauvres eux-mêmes entendent par pauvreté. Cette notion recouvre des mesures de la pauvreté absolue comme les taux de mortalité infantile et juvénile, et des mesures de la pauvreté relative, au regard de normes variables selon les sociétés ${ }^{2}$.

Le Sommet mondial pour le développement social qui a eu lieu à Copenhague en 1995 et les objectifs de développement du millénaire partent du principe que la pauvreté est pluridimensionnelle. De même, la Banque mondiale définit la pauvreté comme une situation inacceptable de dénuement en termes d'opportunités économiques, d'éducation, de santé et de nutrition, caractérisée en outre par l'absence d'intégration dans la société

La pauvreté est perçue de plusieurs manières différentes... 
i.. renvoyant à divers types de capacités individuelles et collectives... et par l'insécurité. Le Programme des Nations unies pour le développement (PNUD) a créé deux notions connexes : celle de développement humain, défini comme un processus qui élargit les choix des populations, notamment en termes de liberté, de dignité, d'estime de soi et de statut social, et celle de pauvreté humaine, qui renvoie à la privation de capacités fondamentales telles qu'une vie longue et saine, l'accès au savoir et aux ressources économiques et la participation à la vie locale.

Si ces multiples facettes de la pauvreté sont le reflet de la réalité des pauvres, elles rendent l'identification des populations concernées et le suivi des progrès encore plus compliqués. Certaines des dimensions de la pauvreté qui viennent d'être évoquées ne peuvent pas être mesurées correctement, ou ne s'inscrivent pas dans la même logique que d'autres. En outre, il peut y avoir un risque que les politiques et les activités acquièrent une sorte de « label pauvreté », sans qu'elles se traduisent pour autant par une réelle évolution vers une action plus résolument tournée vers les pauvres.

Les Lignes directrices pour la réduction de la pauvreté tiennent compte de ces deux types de difficultés. Elles proposent une définition de la pauvreté à la fois concrète et satisfaisante sur le plan théorique, qui la replace dans un cadre plus large englobant à la fois les causes de la pauvreté et les mesures qui peuvent être prises pour y remédier. Si l'on veut parvenir à faire reculer la pauvreté, on ne saurait faire l'économie d'une analyse de ses causes et d'une évaluation de l'efficacité probable des divers instruments d'action.

\section{Les principales dimensions de la pauvreté}

Une définition adéquate de la pauvreté doit recouvrir tous les domaines essentiels dans lesquels hommes ou femmes connaissent des privations et sont perçus comme privés de capacités fondamentales, dans des sociétés et des contextes particuliers différents. Elle doit s'étendre aussi aux liens de causalité entre les principales dimensions de la pauvreté, et reconnaître l'importance centrale des aspects liés à l'égalité homme-femme et de l'instauration d'un développement écologiquement viable (figure 1).

Les capacités économiques renvoient à l'aptitude à générer un revenu, à consommer et à détenir des actifs, autant d'éléments déterminants pour la sécurité alimentaire, le bien-être matériel et le statut social. Ces aspects sont souvent mis en avant par les pauvres, au même titre que la sécurité d'accès aux ressources productives financières et matérielles comme la terre, les outils et le bétail, la forêt et les eaux de pêche, le crédit ou un emploi décent.

Les capacités humaines se rapportent à la santé et à l'éducation, ainsi qu'à l'accès à la nourriture, à l'eau propre et au logement, autant de facteurs essentiels au bien-être des individus, et décisifs pour l'amélioration de leurs moyens de subsistance. La maladie et l'illettrisme sont des obstacles à l'emploi productif et, par voie de conséquence, au développement des capacités, économiques et autres, indispensables pour se sortir de la pauvreté. Savoir lire et écrire facilite la communication, condition sine qua non de toute participation à la vie sociale et politique. L'éducation, des filles en particulier, est considérée comme le moyen à lui seul le plus efficace de vaincre la pauvreté et certaines de ses principales causes, par exemple la maladie, notamment le sida, et la fécondité excessive.

Les capacités politiques recouvrent la possibilité de faire respecter ses droits en tant qu'être humain, de se faire entendre et d'exercer une certaine influence sur les politiques publiques et les priorités politiques. La négation des libertés politiques fondamentales ou des droits de l'homme est une des principales caractéristiques de la pauvreté. Elle se manifeste notamment sous la forme d'actions arbitraires, injustes, voire violentes, de la part de la police ou d'autres autorités publiques, qui alimentent le sentiment déjà 
Figure 1. Interaction entre les divers aspects de la pauvreté et du bien-être

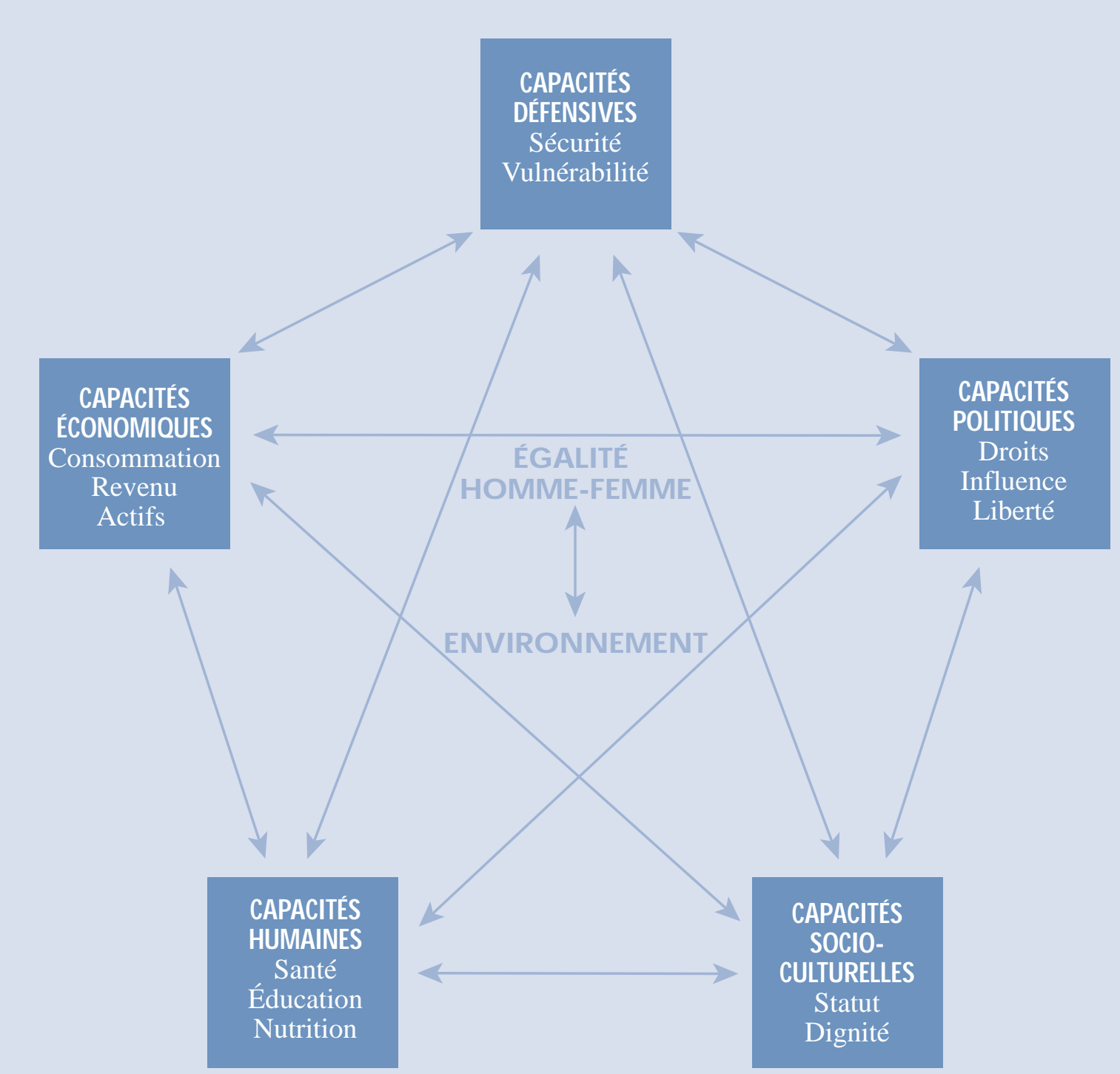

aigu de crainte régnant chez les pauvres. Le fait de ne disposer d'aucun pouvoir accentue encore les autres dimensions de la pauvreté. Les politiquement faibles n'ont pas suffisamment de poids pour influer sur les réformes, et ne bénéficient pas non plus d'une sécurité d'accès aux ressources dont ils auraient besoin pour se sortir de la pauvreté.

Les capacités socioculturelles ont trait à l'aptitude à devenir un membre apprécié d'une communauté. Elles renvoient au statut social, à la dignité et à d'autres conditions d'ordre culturel indispensables pour faire partie d'une société, éléments auxquels les pauvres eux-mêmes attachent une grande importance. Les évaluations participatives de la pauvreté indiquent que l'isolement géographique et social est le principal corollaire de la pauvreté pour la population de nombreuses communautés locales, les autres dimensions de la pauvreté étant perçues comme des facteurs aggravants. 
...interdépendantes...

... mais entre lesquelles il est utile d'établir une distinction à des fins $d$ 'analyse et d'orientation de l'action.

Faire reculer la pauvreté nécessite de porter une attention particulière...

... à l'égalité entre les hommes et les femmes...
Les capacités défensives sont celles permettant aux individus de résister à des chocs économiques et extérieurs. Insécurité et vulnérabilité sont des caractéristiques essentielles de la pauvreté, étroitement liées aux autres dimensions de la pauvreté. Les pauvres soulignent que la faim et l'insécurité alimentaire sont pour eux des préoccupations fondamentales, au même titre que d'autres dangers comme la maladie, la criminalité, la guerre et la spoliation. La pauvreté est un état qui, dans une large mesure, est vécu de façon intermittente en fonction des saisons et des chocs extérieurs tels que catastrophes naturelles, crises économiques ou conflits violents, en fonction. Il faut pouvoir définir la pauvreté de façon dynamique pour rendre compte du fait que la pauvreté a ses entrants et ses sortants. Les pauvres d'aujourd'hui ne sont qu'en partie les mêmes que ceux d'hier ou de demain. Pour certains la pauvreté est un fléau chronique ou hérité, pour d'autres elle est un épisode temporaire ou transitoire.

Les relations que symbolisent les flèches dans la figure 1 sont significatives. Chaque encadré représente une dimension importante de la pauvreté, qui influe sur les toutes autres et est influencée par toutes les autres. Les membres d'un foyer peuvent consommer peu et devoir leur vulnérabilité en partie au fait qu'ils ne détiennent guère d'actifs, en raison souvent de l'insuffisance de leur revenu, de leur mauvaise santé et de leur faible niveau d'instruction ou de la perte de leurs rares actifs productifs à la suite de chocs externes. L'absence de reconnaissance des droits de l'homme et des libertés politiques laisse supposer un risque de conflits violents. La vulnérabilité et l'exclusion sociale limitent les capacités humaines et politiques, d'où une diminution des revenus et des actifs, avec les conséquences qui en découlent. Le fait que les différentes dimensions de la pauvreté sont étroitement imbriquées, même si elle demeurent distinctes et imparfaitement corrélées, est l'une des principales raisons qui milite en faveur de l'adoption d'une définition pluridimensionnelle de la pauvreté.

\section{Liens entre la pauvreté et la problématique homme-femme et l'environnement}

La pauvreté, l'égalité homme-femme et la préservation de l'environnement sont des facettes du développement durable qui se renforcent mutuellement, se complètent et se recoupent les unes les autres. La prise en compte systématique des questions d'égalité homme-femme doit être au cœur de toute action visant à réduire la pauvreté, dans ses diverses dimensions, et à améliorer l'état de l'environnement. Les facteurs environnementaux ont une influence sur les relations entre les hommes et les femmes de même que sur la pauvreté. L'égalité homme-femme et la pérennité de l'environnement constituent en soi des objectifs internationaux de développement. La présente section traite de l'importance des questions relatives à la problématique homme-femme et à l'environnement pour la compréhension de la pauvreté et l'instauration des conditions nécessaires à un recul de la pauvreté.

L'inégalité entre les hommes et les femmes est une constante qui touche toutes les dimensions de la pauvreté vis-à-vis de laquelle hommes et femmes ne sont pas à égalité Les traditions culturelles sont souvent porteuses de préjugés et de discrimination à l'encontre des femmes. Les processus qui mènent à la pauvreté affectent différemment, et à des degrés divers, les hommes et les femmes. La pauvreté est plus fréquente et prend une forme généralement plus aiguë chez les femmes que chez les hommes. Dans les ménages pauvres, les femmes et les filles obtiennent moins que leur part de la consommation privée et des services publics. Elles souffrent fréquemment de la violence des hommes et, pour elles, le risque est plus grand d'être illettrées et politiquement ou socialement exclues de la communauté. C'est pourquoi les moyens qu'ont les femmes de se sortir de la pauvreté ne sont généralement pas les mêmes que ceux des hommes. 
Le rôle des femmes dans les ménages pauvres est déterminant et à la mesure de leur contribution à l'apport des moyens d'existence et des capacités humaines fondamentales. En subvenant aux besoins de leurs enfants, les femmes réduisent le risque de pauvreté pour la génération qui suit. Pourtant, elles ont en général moins facilement accès que les hommes aux actifs générateurs de sécurité et d'opportunités. Les contraintes qui pèsent ainsi sur le potentiel de production des femmes compriment les revenus des ménages et, plus globalement, entravent la croissance économique. L'inégalité entre les hommes et les femmes est donc une cause majeure de pauvreté chez les femmes, et de pauvreté en général.

Les femmes pâtissent d'un manque de temps, lié au nombre de tâches qui leur sont imposées et ne leur laissent guère de latitude pour se reposer ou se consacrer à des activités économiques, sociales ou politiques. Ce manque de temps est une entrave supplémentaire qui, dans de nombreuses sociétés, s'explique par une inégalité structurelle entre les deux sexes, qui n'a pas les mêmes conséquences pour les hommes que pour les femmes.

Les interactions entre l'environnement et la pauvreté sont multiples. La détérioration de l'environnement, tant en milieu rural qu'en milieu urbain, touche en premier lieu les pauvres, et elle est elle-même une conséquence de la pauvreté. Le développement durable et la réduction de la pauvreté exigent que l'on préserve l'intégrité des écosystèmes naturels et que l'on protège leurs fonctions de sauvegarde de la vie le $^{4}$ Les aspects fondamentaux de l'articulation entre environnement et pauvreté sont la sécurité de l'accès à des ressources naturelles dont dépendent un grand nombre de familles pauvres, et les risques pour la santé liés à l'état de l'environnement qui menacent en particulier les femmes et les enfants.

La dégradation des terres, la déforestation et la diminution des stocks de poissons font peser une lourde menace sur les moyens d'existence et la santé des pauvres habitant des zones rurales, sans parler de la pollution de l'air et de l'eau. Pourtant, les pauvres vivant en milieu rural n'ont souvent pas d'autre choix pour vivre que d'utiliser des terres fragiles pour pratiquer une agriculture de subsistance, faire paître leurs troupeaux ou effectuer des coupes de bois, au risque d'aggraver l'érosion des sols. La raréfaction des bonnes terres et de l'eau propre peut attiser l'instabilité sociale et politique et provoquer des conflits aux niveaux local, national et régional, si l'on ne parvient pas à concilier les besoins concurrents des différents utilisateurs dans l'harmonie et l'équité.

Les pauvres, des zones rurales comme des zones urbaines, sont très exposés aux catastrophes naturelles dévastatrices ${ }^{5}$ comme la sécheresse, les inondations, les ouragans ou les typhons, ou encore la montée du niveau de la mer qui menace l'ensemble de la population des petites îles et des zones côtières de faible élévation, en particulier dans les pays pauvres. La fréquence et la gravité de ces catastrophes seront probablement encore amplifiées par le réchauffement de la planète, qui va sans doute s'accélérer. La croissance économique, si elle est importante pour faire reculer la pauvreté dans les pays en développement, amène aussi un accroissement des émissions de gaz à effet de serre à l'origine du changement climatique sur la planète.

Les pauvres habitant dans des zones urbaines vivent souvent dans des conditions sanitaires déplorables et dans des quartiers surpeuplés à proximité de zones contaminées, de sites industriels ou de routes extrêmement fréquentées. Les pauvres qui se sont installés dans ces zones à forte densité démographique sont particulièrement exposés aux catastrophes telles que glissements de terrain ou incendies. Enfin, les fumées qu'inhalent les pauvres des zones aussi bien rurales qu' urbaines dans leur propre logement sont à l'origine de graves problèmes de santé. Les autres risques liés à l'environnement concernent notamment la sécurité sur les lieux de travail, par exemple la sécurité des travailleurs agricoles exposés à des pesticides et autres produits chimiques dangereux ${ }^{6}$.
... et à la préservation de l'environnement. 
Il ex iste diverses sources de données sur la pauvreté...

\section{... mais des informations plus nombreuses et de meilleure qualité restent indispensables.}

\section{Qui sont les pauvres?}

La question de savoir qui sont les pauvres est pertinente à différents niveaux de la société : individu, ménage, quartier, circonscription, région, etc. Les recensements et autres enquêtes nationales auprès des ménages fournissent des données utiles sur le niveau moyen des revenus ou de la consommation, et leur répartition, mais, si l'on ne dispose pas de précisions supplémentaires, les pauvres ne peuvent pas être recensés au moyen des statistiques nationales. La planification de toute action en faveur des pauvres passe par la définition de catégories sociales sur la base de critères comme le sexe, l'appartenance ethnique, la religion ou le milieu culturel, le lieu d'implantation et les moyens de subsistance aussi bien que le type de ménage, c'est-à-dire le nombre de membres du foyer et leur répartition par classes d'âge, ainsi que le sexe du chef de famille.

Pour établir des profils, les bilans de pauvreté au niveau national exploitent des données tirées essentiellement d'enquêtes auprès des ménages, mais aussi des indicateurs nationaux du développement humain. Les bilans de pauvreté participatifs enrichissent les profils pluridimensionnels de données quantitatives et qualitatives. Même si, dans certains cas, ils sont plus indiqués lorsqu'il s'agit de définir des stratégies et des actions, leur coût est également beaucoup plus élevé.

Les différentes facettes de la pauvreté et leurs mesures peuvent être en décalage, ce qui peut rendre l'identification des pauvres plus difficile. A titre d'exemple, des individus peuvent souffrir de pauvreté monétaire même si leurs enfants, y compris les filles, fréquentent l'école primaire et si les taux de mortalité infantile et maternelle ont pu être réduits dans le pays concerné conformément aux objectifs de développement du millénaire. De même, il arrive que des personnes illettrées gagnent plus que le fatidique « un dollar » par jour, etc. Plus on dispose d'informations sur ces différences et ces interactions réciproques, plus il est possible d'affiner la conception des politiques et des actions à mener.

Malgré les difficultés rencontrées pour mesurer précisément la pauvreté, des informations provenant de sources différentes peuvent souvent être utilisées pour identifier les pauvres. Les catégories sociales auxquelles est associée la grande pauvreté dans plusieurs de ses facettes englobent les groupes indigènes, les minorités et les exclus de la société, les réfugiés et les personnes déplacées, les handicapés mentaux ou physiques et les victimes du VIH/sida. Les femmes et les enfants sont particulièrement vulnérables, notamment les veuves âgées, les familles privées de tout soutien dont le chef est une femme ou un enfant, et les enfants des rues. Ces groupes comptent parmi les plus pauvres d'entre les pauvres dans bien des sociétés et méritent une attention spéciale dans toute action stratégique visant à combattre la pauvreté.

\section{Comment mesurer la pauvreté et en suivre l'évolution?}

L'étape suivante consiste à mesurer les différentes dimensions de la pauvreté. Une quantification est nécessaire pour déterminer dans quelle mesure les objectifs fixés sont atteints, pour évaluer l'impact de politiques ou de programmes particuliers, et pour identifier les pauvres. Une pratique optimale consisterait à collecter des données qui permettent de différencier les populations par sexe, par âge et par catégorie sociale ${ }^{7}$. L'adéquation des divers outils de mesure de la pauvreté dépend de l'accessibilité des données et de la finalité de la mesure. Il est plus coûteux et plus long de mesurer et de quantifier les dimensions les moins tangibles de la pauvreté. 
Les indices composites, englobant à la fois l'aspect monétaire et d'autres dimensions de la pauvreté, peuvent fournir des mesures quantitatives comparables plus solides que les indicateurs renvoyant à une seule dimension. Les plus connus ont été mis au point par le PNUD dans le cadre de son Rapport mondial sur le développement humain ${ }^{8}$. C'est essentiellement grâce à eux qu'ont pu être mises en évidence la pluridimensionnalité et la gravité du problème de la pauvreté. Toutefois, le choix des indicateurs et les coefficients de pondération qui leur sont attribués ont dans une certaine mesure un caractère arbitraire, et les interactions réciproques ne sont pas prises en compte. C'est pourquoi les mesures discrètes de la pauvreté demeurent plus utiles pour certains aspects de la planification.

Chaque type de mesure a son utilité spécifique : les indicateurs relatifs, contextuels, qualitatifs et pluridimensionnels sont mieux adaptés pour appréhender une situation particulière et intervenir efficacement en conséquence, mais le sont moins à des fins de comparaison ou pour suivre l'évolution de la pauvreté en général et en évaluer l'impact, ce pour quoi il vaut mieux recourir à des mesures absolues, simples et chiffrées (figure 2).

La partie supérieure de la figure 2 représente la mesure simple de la consommation ou du revenu dépensé, qui peut être obtenue, dans un grand nombre de pays, à partir des enquêtes auprès des ménages. Ce type de mesure est utile à des fins d'analyse comparative de la pauvreté sur une période donnée dans un pays et entre différents pays, et pour suivre l'évolution globale de la pauvreté. En d'autres termes, il s'agit d'une vue d'ensemble de la pauvreté. La partie médiane comporte des indices composites.

La partie inférieure donne les fondements indispensables à la mesure de la pauvreté dans ses diverses dimensions. C'est un niveau de mesure qui se rapproche plus du terrain et qui, de ce fait, est d'une plus grande utilité dans la planification détaillée et le suivi. Des statistiques nationales sur le développement humain sont collectées régulièrement dans le cadre d'enquêtes et présentées dans des tableaux généraux dans le Rapport sur le développement humain du PNUD, et dans le Rapport sur le développement dans le monde de la Banque mondiale. En ce qui concerne les autres dimensions de la pauvreté, il n'existe aucune méthode valable pour normaliser et quantifier les mesures qui permettraient la comparaison. Plusieurs pays ont néanmoins entrepris des bilans de pauvreté participatifs qui fournissent des informations qualitatives et pluridimensionnelles très utiles.

L'adoption d'une approche étroite pour mesurer la pauvreté permet l'identification et l'analyse statistique des ménages qui vivent en dessous d'un seuil de pauvreté absolue, correspondant à une norme minimale de nutrition et de consommation, ce qui est une étape indispensable pour suivre l'évolution du nombre aussi bien que de la part relative des pauvres ${ }^{9}$ sur une période donnée et entre pays, ainsi que la gravité et la densité de la pauvreté. Les seuils de pauvreté les plus fréquemment utilisés pour établir des comparaisons internationales sont fixés à un, deux et quatre dollars par jour respectivement pour les pays à faible revenu, les pays à revenu intermédiaire et les économies en transition. Nombre de pays appliquent leurs propres seuils qui reflètent les considérations sociales, économiques et climatiques intervenant dans la détermination du revenu minimum acceptable.

Une fois bien établi ce que l'on entend par pauvreté, et comment il est possible de la mesurer, il convient, pour élaborer une stratégie de lutte contre la pauvreté, de définir des indicateurs de référence et des objectifs. Les gouvernements doivent fixer des objectifs de réduction de la pauvreté et déterminer des moyens de les chiffrer et d'en assurer le suivi. Les objectifs et les indicateurs de référence, qui doivent être établis en fonction des sources de données disponibles pour la période couverte, doivent aussi être
... qui ont toutes leur utilité selon que l'on veut...

... programmer une action spécifique de lutte contre la pauvreté,...

... procéder à des comparaisons générales...

... ou fixer des objectifs et des étalons. 


\section{Figure 2. Mesure des différentes dimensions de la pauvreté}

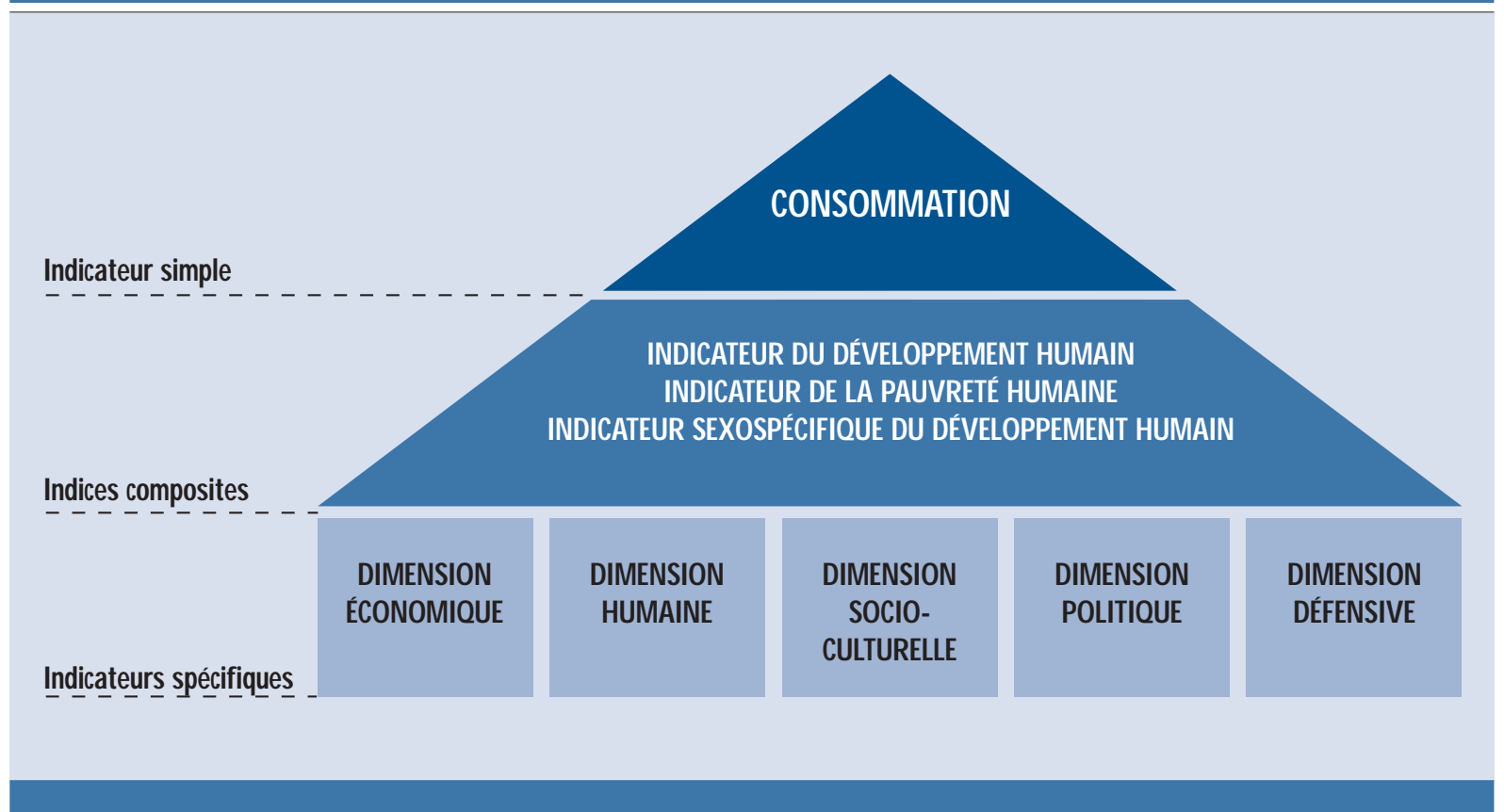

Les causes

habituelles de la pauvreté sont bien connues... compatibles avec les réalités économiques, politiques et socioculturelles de chaque pays et préciser ce que recouvre exactement la réduction de la pauvreté. Ils doivent renvoyer aux objectifs de développement du millénaire, même si les pays peuvent se fixer des objectifs propres fondés sur des indicateurs différents et assortis d'échéances différentes.

Il est essentiel d'associer aux indicateurs de portée nationale d'autres indicateurs permettant de ventiler les résultats en fonction de différentes catégories de population, définies sur la base de critères tels que le sexe, l'âge, la catégorie sociale, la région géographique ou administrative, le caractère rural ou urbain de la zone d'habitation. Mettre ainsi en évidence les disparités est un bon moyen d'attirer l'attention sur certains aspects importants de la pauvreté, et notamment d'alerter rapidement sur des risques de conflit éventuels. La participation des populations au processus de fixation des objectifs et d'établissement des indicateurs peut faire naître un consensus au niveau national sur la réduction de la pauvreté. Il est capital de disposer de données fiables pour analyser les résultats dont les pouvoirs publics peuvent être tenus pour responsables, et pour suivre l'évolution de la situation des diverses catégories de pauvres.

\section{Comment expliquer la persistance de la pauvreté ?}

La formulation de stratégies de lutte contre la pauvreté comporte encore deux étapes : identifier et hiérarchiser les causes de chaque dimension de la pauvreté dans une région ou un pays donné, et mettre au point des politiques et des mesures visant à y remédier. La présente section est consacrée à la première de ces étapes.

Parmi les causes profondes de la pauvreté, certaines sont liées à des facteurs immuables comme le climat, la géographie et l'histoire. Les carences dans la gestion des affaires publiques, qui ne sont, elles, pas une fatalité, sont porteuses de tout un ensemble de facteurs qui perpétuent la pauvreté. La corruption généralisée et la présence d'élites soucieuses de préserver leur rente de situation, le non-respect des droits de l'homme, 
la faiblesse des institutions et le manque d'efficacité des administrations, l'absence de cohésion sociale et de volonté politique d'entreprendre des réformes sont autant de traits communs aux mauvais systèmes de gestion des affaires publiques et sont peu propices au développement durable et à la réduction de la pauvreté. Dans des cas extrêmes, mais malheureusement pas rares, les carences dans la gestion des affaires publiques peuvent entraîner des conflits violents et un effondrement de l'État. Les inégalités entre hommes et femmes ou entre catégories sociales et économiques figurent également parmi les principaux facteurs favorisant la pérennisation de la pauvreté, de même que la détérioration de l'environnement et l'explosion démographique. Le VIH/sida est un fléau apparu assez récemment qui intervient de façon cruciale dans la pauvreté et touche particulièrement les jeunes femmes et les enfants, parmi lesquels il fait beaucoup d'orphelins.

Ces différents facteurs peuvent tous contribuer à une insuffisance de la croissance économique, laquelle est une des causes premières de la pauvreté. D'autres éléments interviennent également, parmi lesquels les dysfonctionnements dans la gestion des affaires publiques et le manque d'équité, l'échec des politiques économiques mises en œuvre et les défaillances du marché, la fuite des capitaux, la faiblesse de l'épargne et de l'investissement, ou encore les distorsions induites par certaines mesures incitatives, qui tous concourent à la baisse de la productivité et des revenus. Une inflation galopante s'apparente à un prélèvement extrêmement lourd qui vient ponctionner les revenus et l'épargne réels des pauvres. L'état déplorable des infrastructures physiques et sociales est à la fois une conséquence et un facteur aggravant de la stagnation, voire du déclin, de l'économie. Le protectionnisme pratiqué sur les marchés d'exportation potentiels, ainsi d' ailleurs que l'instabilité et la tendance à la détérioration des termes de l'échange, sont des causes économiques extérieures de pauvreté. Le surendettement, sur le plan tant intérieur qu'international, est lui aussi une circonstance aggravante.

Il est bien plus complexe de classer par ordre d'importance les principales causes de la pauvreté dans un pays donné que d'identifier les principaux groupes sociaux parmi lesquels se trouvent les pauvres, ou d'établir un descriptif des groupes victimes de la pauvreté. C'est pourquoi l'entreprise est rarement tentée, bien qu'elle soit essentielle pour combattre efficacement la pauvreté. Toutes les politiques adoptées par les autorités nationales et les stratégies retenues par les organismes de développement pour lutter contre la pauvreté doivent s'appuyer sur au moins une ébauche de hiérarchisation et d'évaluation des causes de la pauvreté. La planification stratégique suppose que l'on prenne des décisions, que l'on fasse des choix, que l'on définisse des priorités, et le classement des instruments d'action en fonction de leur incidence probable sur la pauvreté est la marque d'une démarche mûrement réfléchie.

La formulation d'une stratégie de lutte contre la pauvreté est une tâche pleine d'incertitudes. Les dirigeants, le personnel des organismes d'aide et les conseillers doivent s'assurer que leurs jugements sont au moins des estimations fondées, c'est-àdire qu'ils s'appuient sur toutes les données et analyses existantes, ou pouvant facilement être obtenues au niveau local ${ }^{10}$.

\section{Quelles sont les mesures à prendre ?}

Les stratégies de lutte contre la pauvreté et de développement durable doivent converger et servir de plate-forme commune aux programmes et projets de coopération pour le développement. Le développement durable présente de multiples facettes : il recouvre des aspects économiques, sociaux, environnementaux et institutionnels, et appelle une approche intégrée, transcendant le plus souvent les frontières sectorielles et institutionnelles. Toute stratégie visant à réduire réellement et durablement la pauvreté
... mais il est essentiel de déterminer et de hiérarchiser ses causes spécifiques dans chaque pays. 
Une approche globale est indispensable ; elle doit intégrer...
... des réformes économiques destinées à instaurer une croissance favorable aux pauvres,...

... à améliorer la gestion des affaires publiques et renforcer les institutions,.... doit couvrir les domaines d'action suivants, qui sont complémentaires et dont l'énumération ne suit pas un ordre de priorité ${ }^{11}$ :

a) Croissance économique fondée sur une dynamique de réduction de la pauvreté : rythme et qualité.

b) Autonomisation, renforcement des droits et gestion des affaires publiques fondée sur une dynamique de réduction de la pauvreté.

c) Services sociaux de base à l'appui du développement humain.

d) Sécurité humaine : réduire la vulnérabilité et gérer les crises.

e) Prise en compte systématique de la problématique homme-femme et promotion de l'égalité entre les sexes.

f) Prise en compte systématique de la nécessité d'assurer la pérennité de l'environnement par l'instauration de modes d'utilisation durables des moyens d'existence.

\section{Croissance économique fondée sur une dynamique de réduction de la pauvreté : rythme et qualité}

La croissance économique est capitale pour faire reculer la pauvreté, et le rythme et la qualité, c'est-à-dire la composition, la répartition et la durabilité, de cette croissance sont des facteurs particulièrement décisifs. Le manque de moyens financiers est une dimension essentielle de la pauvreté en même temps qu'une cause majeure des autres formes de privation associées à la pauvreté. Une croissance équitable du revenu national atténue la pauvreté monétaire de la plupart des ménages. De leur côté, des stratégies efficaces de réduction durable de la pauvreté suscitent une augmentation du revenu de la plupart des ménages, et du PIB national.

La croissance de l'économie nationale peut, par sa nature, contribuer plus ou moins à faire reculer la pauvreté, favoriser l'égalité entre les hommes et les femmes et instaurer un développement durable ${ }^{12}$. De façon générale, il existe un lien étroit entre croissance économique et réduction de la pauvreté, la fréquence moyenne aussi bien que la gravité de la pauvreté tendant à fléchir lorsqu'il y a croissance ${ }^{13}$. La croissance économique peut créer des opportunités pour les pauvres, mais la pauvreté ne diminuera que si les conditions sont réunies pour qu'ils puissent mettre à profit ces opportunités.

Les stratégies et politiques efficaces pour enclencher une croissance bénéfique pour les pauvres diffèrent selon les pays en fonction de leur dotation en ressources, de leur niveau de technologie et de capital humain, et du contexte historique, institutionnel et socioculturel. En règle générale, une économie de marché concurrentielle constitue un terrain favorable à l'accroissement de la productivité du secteur privé, de l'épargne et de l'investissement, qui à son tour stimule la croissance économique, sous réserve toutefois que les conditions fondamentales suivantes soient réunies :

- Un cadre composé d'institutions publiques, administratives, juridiques et financières propice à une bonne gestion du secteur public et des entreprises et à l'instauration d'un développement durable.

- La stabilité macro-économique et politique.

- Des infrastructures matérielles et sociales adaptées et accessibles à toutes les catégories de la population.

L'accès des pauvres, hommes et femmes, aux ressources telles que la terre, les financements et les capacités humaines. 
- Des formes de production à forte intensité de main-d'œuvre.

- Des politiques sociales favorisant la cohésion, la mobilité, la protection des individus, la redistribution des revenus et l'égalité homme-femme.

On ne peut améliorer les chances qu'ont les pauvres, hommes et femmes, de se sortir de la pauvreté sans mettre en place un cadre juridique et institutionnel satisfaisant. Il est essentiel d'instituer des systèmes de gouvernement d'entreprise de nature à instaurer un environnement propice à l'investissement privé, et un cadre réglementaire garantissant à la fois la concurrence et la stabilité du système, en particulier dans le secteur financier. Des réformes structurelles, au niveau des politiques, des incitations et des institutions, sont souvent indispensables dans des domaines tels que la gestion budgétaire, les échanges, le marché du travail, le secteur financier, l'infrastructure et l'utilisation des sols.

La stabilité macro-économique, alliée à une faible inflation, à une relative stabilité des prix et de la production, à des marchés concurrentiels efficients et à des mesures de nature à favoriser l'épargne et l'investissement, privés et publics, sont autant d'éléments déterminants. L'action des pouvoirs publics doit viser à concourir à l'instauration d'un environnement propice à l'investissement et à la croissance du secteur privé, condition indispensable pour que naissent des emplois. Il faut établir un judicieux équilibre et un partenariat entre les trois sphères de la société : pouvoirs publics, entreprises et société civile.

Enclencher une croissance bénéfique aux pauvres requiert l'adoption de politiques et des programmes permettant aux pauvres d'exploiter leurs propres atouts et capacités pour améliorer et pérenniser leurs moyens d'existence, par exemple des programmes de micro-crédit, ou une politique visant à encourager la production de biens et de services à forte intensité de main-d'œuvre. Les pouvoirs publics doivent aussi s'attaquer à l'inégalité des chances dont sont victimes les pauvres en améliorant leur accès aux services sociaux de base, notamment dans les domaines de l'éducation et de la santé, un tel investissement dans le capital humain étant essentiel pour asseoir largement la croissance. Enfin, les politiques budgétaires, tant en matière de dépenses qu'en matière de recettes, doivent être conçues de manière à appuyer la lutte contre la pauvreté.

Pour les petites économies pauvres, la mondialisation offre un grand potentiel de réduction de la pauvreté. Ces économies sont toutefois les plus exposées aux perturbations économiques que peut induire l'ouverture, comme un infléchissement soudain des mouvements de capitaux à court terme ou une détérioration brutale des termes de l'échange. C'est pourquoi tout processus d'intégration requiert une gestion prudente pour que les pauvres puissent bénéficier d'une croissance tirée par les échanges. Cela suppose d'améliorer les capacités et d'étudier les débouchés commerciaux tout en intervenant pour atténuer les risques de la mondialisation : sorties de capitaux, dégradation de l'environnement, chômage ou non-respect des normes fondamentales du travail, par exemple. L'intégration régionale peut être une étape intermédiaire sur la voie de la libéralisation des échanges permettant aux économies faibles de s'adapter progressivement à des marchés plus vastes et plus concurrentiels.

L'inégalité des actifs et des revenus, en fonction du sexe ou d'autres critères (groupe ethnique, catégorie sociale, implantation régionale, etc.), est un obstacle majeur à la réduction de la pauvreté. L'inégalité a des retombées négatives multiples sur la pauvreté dans la mesure où elle freine la croissance du PIB et amoindrit les effets de cette dernière sur la pauvreté. Plus les inégalités sont marquées et tendent à se creuser, moins les pauvres ont d'influence sur l'orientation de l'action publique et plus s'accroissent les risques de conflit et de violence. Une intervention des pouvoirs publics pour réduire les inégalités

\section{... à ralentir I'inflation et stimuler le secteur privé,...}




\section{... à réduire les inégalités et améliorer l'accès des populations pauvres aux actifs et aux services sociaux, en particulier dans les zones rurales,...}

...et à s'attaquer au problème complexe de la pauvreté urbaine. est primordiale, mais nécessite, ce qui requiert du doigté, la constitution de coalitions politiques visant à contrecarrer les intérêts acquis. Les organismes de développement peuvent faciliter les réformes en instaurant un dialogue sur les politiques à suivre et en apportant un concours technique et financier à des changements structurels favorables aux pauvres, une réforme foncière par exemple ${ }^{14}$.

Une accélération de la croissance améliore les perspectives de réduction de la pauvreté dans tous les pays, mais pour que s'opère une réduction de moitié de la pauvreté monétaire, la croissance doit être en moyenne deux fois plus rapide dans les pays caractérisés par de fortes inégalités que dans ceux où les inégalités sont faibles. Cela étant impossible, il faut donc rendre la croissance plus équitable pour que soit atteint l'objectif international de développement concernant la réduction de la pauvreté monétaire ${ }^{15}$.

Dans les pays en développement, la grande majorité des pauvres vivent encore dans les régions rurales, où l'accès à la santé, à l'éducation, à l'alimentation et aux marchés est souvent plus difficile que dans les zones urbaines. Et le fossé entre les foyers ruraux et urbains ne se comble pas. La proportion de pauvres augmente rapidement dans les zones urbaines ${ }^{16}$. La pauvreté rurale et les opportunités plus nombreuses qu'offrent les agglomérations urbaines incitent les pauvres à un exode vers les villes, d'où une aggravation du chômage et de la pauvreté dans les zones urbaines. Favoriser un développement rural (passant ou non par l'agriculture) qui profite aux pauvres est donc une priorité pour faire reculer globalement la pauvreté.

Des mesures complémentaires doivent être prise pour lutter contre la pauvreté urbaine et la pauvreté rurale. Les zones rurales et urbaines entretiennent des liens qui suivent la trajectoire des familles, des migrations, du commerce et des remises de fonds, et ces liens ont leur importance pour favoriser une croissance économique qui profite aux pauvres. En outre, l'agriculture urbaine procure des moyens d'existence à un nombre considérable de pauvres vivant en milieu citadin. L'avènement d'une croissance durable et équilibrée entre hommes et femmes dans les secteurs de la petite agriculture et des services ruraux est un moyen particulièrement efficace de combattre la pauvreté dans les villes et à la campagne car il permet de tisser des liens solides en amont et en aval grâce à l'accroissement de la demande et de l'offre, lequel stimulera l'augmentation des revenus et de l'emploi.

Dans les zones rurales, il convient d'assurer aux pauvres un meilleur accès aux ressources dans des domaines importants bien que quelque peu négligés, à savoir : les actifs physiques et financiers, la technologie et les ressources naturelles (terre et eau), les marchés et les institutions ${ }^{17}$. Soutenir la recherche et la diffusion de semences améliorées et appuyer le développement de la micro-irrigation et du micro-crédit et la réalisation d'ouvrages publics sont autant de moyens utiles d'aider les pauvres des zones rurales, hommes et femmes, à sortir de la pauvreté.

La pauvreté urbaine renvoie à une réalité complexe et lourde d'enjeux. Il est primordial de définir une politique d'aménagement du territoire au service d'un développement durable des zones urbaines. L'expansion rapide des villes dans les pays en développement, en particulier d'Afrique et d'Amérique latine, suscite de graves problèmes pour l'environnement et accroît l'insécurité sociale et matérielle tout en privant les habitants des réseaux traditionnels d'aide sociale. Il faut donc offrir des services sociaux et des services d'utilité publique de qualité et mettre en place des programmes spéciaux destinés à améliorer la productivité des pauvres, hommes et femmes. Parallèlement, il convient d'éviter les subventions susceptibles d'avoir des effets de distorsion, lesquelles risqueraient de conduire à un afflux encore plus important de 
pauvres venus des campagnes et à une nouvelle aggravation de la pauvreté dans les villes. Le développement des marchés par la réforme des institutions et l'amélioration des infrastructures est un élément déterminant. Être propriétaire de son terrain est important, pour les habitants des villes comme pour ceux des régions rurales, à la fois en tant que gage de sécurité et pour avoir une garantie à offrir si l'on souhaite monter une petite affaire. Le secteur informel contribue pour beaucoup à contenir la pauvreté urbaine, mais il faut accorder davantage de place au respect de normes minimales dans les domaines du travail et de l'environnement.

\section{Autonomisation, renforcement des droits et gestion des affaires publiques fondée sur une dynamique de réduction de la pauvreté}

La pauvreté va souvent de pair avec l'absence de moyens d'action, l'injustice et l'exclusion de la vie sociale, en raison de la discrimination dont font l'objet les pauvres, et, plus généralement, la négation des droits essentiels, y compris les droits politiques. La démarginalisation des pauvres, femmes et hommes, requiert un mode de gestion démocratique des affaires publiques fondé sur la participation populaire à l'élaboration des politiques et à la conception et la mise en œuvre des programmes, une société civile comptant des organisations représentatives de tous les segments de la population et le respect des droits de l'homme et de l'état de droit. L'indépendance des médias peut aussi contribuer largement à l'émergence d'une culture démocratique.

L'autonomisation passe par un renforcement de la capacité des pauvres, hommes et femmes, d'exercer une influence sur les mécanismes politiques et sociaux qui ont des répercussions sur le cours de leur vie. Certaines conditions doivent être remplies pour que les pauvres puissent faire valoir les droits qui sont reconnus à tout homme et se mobiliser pour faire entendre leur voix. La démocratisation est censée permettre aux hommes et aux femmes d'exiger le respect de leurs droits, mais trop souvent les puissants et les privilégiés, désireux d'affermir encore leur position, usurpent le pouvoir au sein des institutions politiques à leur profit exclusif ${ }^{18}$.

Les approches de la réduction de la pauvreté fondées sur les droits retiennent de plus en plus l'attention : elles raccordent l'autonomisation des pauvres, hommes et femmes, à l'ensemble des normes et principes énoncés dans les accords internationaux relatifs aux droits de l'homme ${ }^{19}$. Elles s'attaquent aux causes de la pauvreté en identifiant ceux qui ont des droits et ceux qui ont des devoirs dans l'application des droits de l'homme, qu'il s'agisse des droits civils, culturels, politiques, sociaux ou économiques. L'accent mis sur les droits de l'homme montre bien que la justice est une question de droits, pas de charité. Reconnaître ce principe, pour les uns, et savoir comment revendiquer ses droits, pour les autres, peut aider les pauvres et les groupes socialement exclus à retrouver confiance et faciliter une participation plus active de leur part au processus de développement.

Toute approche fondée sur les droits suppose un renforcement de la capacité des tribunaux et autres institutions de promouvoir et protéger les droits universels des hommes et des femmes, y compris le droit de disposer d'une information adéquate, d'obtenir un travail décent et de s'organiser en syndicats. Un processus politique démocratique et des systèmes économiques, juridiques et judiciaires efficaces sont des éléments importants pour améliorer l'environnement général et le rendre propice à la démarginalisation des pauvres et des groupes socialement exclus, ce qui nécessite la transparence et l'efficacité des structures de gestion des affaires publiques, des institutions démocratiques et comptables de leurs actes et la liberté de la presse. Des efforts visant à améliorer la gestion des affaires publiques et à enrayer la corruption en renforçant l'obligation de rendre des comptes et en privilégiant davantage la notion de service dans le secteur public sont indispensables. Ils doivent être étayés par des réformes de la
Le souci des pauvres dans la gestion des affaires publiques est capital...

... afin de renforcer leur participation aux processus démocratiques et le respect de leurs droits essentiels, y compris les droits politiques,...

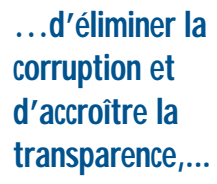


... autant d'éléments que peut faciliter une décentralisation démocratique. fonction publique destinées à adapter le système des incitations à l'intention des fonctionnaires et à leur garantir notamment le versement régulier de salaires décents.

La délégation de pouvoir et la participation accrue des pauvres, hommes et femmes, aux échelons locaux ou régionaux d'administration jouent en faveur de la responsabilisation et de la prise de décisions favorables aux pauvres. La réforme doit également, ce qui va de pair, porter sur la décentralisation administrative qui peut mettre un frein à la grande corruption et améliorer l'efficience des services publics. Cela dit, dans des sociétés très inégalitaires, où les structures d'organisation sociale des pauvres sont en outre déficientes, la décentralisation risque surtout d'aggraver les abus commis par les élites locales dont les décisions ne sont plus soumises à aucun contrôle. La décentralisation, pour profiter aux pauvres, doit s'accompagner d'un renforcement des structures locales de gestion des affaires publiques et des obligations de comptes envers les citoyens.

\section{Services sociaux de base à l'appui du développement humain}

Par développement humain, il faut entendre le renforcement des capacités humaines et l'élargissement de l'éventail des choix offerts aux individus concernant ce qu'ils font et peuvent faire de leur vie. Le développement humain passe par l'accroissement du revenu et de la richesse ainsi que par l'accès à une alimentation adéquate, une eau propre, des services médicaux, des établissements scolaires et des moyens de transport de qualité pour un prix abordable, un logement et un travail décents et des moyens d'existence sûrs. Une croissance économique soutenue fondée sur une dynamique de réduction de la pauvreté permet d'accroître le revenu et la consommation des pauvres, hommes et femmes, et génère en outre des ressources susceptibles d'être dirigées vers les services sociaux. Un large accès à des services sociaux de qualité est source de croissance économique en ce qu'il permet d'accroître les capacités productives de la population.

Le VIH/sida et d'autres maladies de plus en plus répandues parmi les pauvres, comme le paludisme et la tuberculose, compromettent la cohésion sociale, la croissance économique et, partant, la réduction de la pauvreté. Le sida soulève désormais des problèmes absolument cruciaux pour le développement de l'Afrique et risque fort de faire de même sous peu en Asie, où la population est beaucoup plus nombreuse. La reconnaissance, par les plus hautes sphères politiques, de l'importance majeure de ce fléau et de questions connexes telles que les droits et la santé génésiques, est indispensable au succès de toute stratégie de développement humain et de lutte contre la pauvreté.

Pour vivre vieux, en bonne santé et dans des conditions gratifiantes, il faut avoir accès à des services sociaux publics de qualité qui fournissent des soins préventifs et curatifs et un enseignement scolaire ou extra-scolaire. L'enseignement de base, pour les filles, les garçons, les femmes et les hommes, est le facteur qui contribue le plus, à lui seul, à assurer le développement humain, à faire reculer la pauvreté, à limiter la propagation du sida et à réduire le taux de fécondité. Les hommes et les femmes pauvres n'ont souvent pas accès à des services sociaux adéquats à cause de l'insuffisance des ressources publiques qui y sont allouées et de leur mauvaise gestion. Des mesures spéciales peuvent faciliter une distribution plus large des services de base et assurer aux pauvres, femmes, hommes et enfants, un meilleur accès à ces services. Il faut rechercher soigneusement et mettre au point des méthodes, favorables aux pauvres, de financement des services sociaux publics par l'impôt et, dans certains cas, par des redevances d'utilisation, et en suivre la mise en œuvre aux fins de garantir l'accès à un prix abordable à des prestations de qualité. 
La part des dépenses publiques allouée, dans les pays pauvres, à l'enseignement primaire et à la santé sert souvent d'indicateur pour apprécier l'attention portée à la lutte contre la pauvreté dans les politiques gouvernementales ${ }^{20}$. Le niveau des dépenses publiques a certes son importance, mais un surcroît de ressources n'aura d'effet que s'il est utilisé efficacement. Il est capital d'améliorer l'affectation des dépenses sociales dans le cadre de grandes politiques et stratégies sectorielles tenant compte des arbitrages à opérer, par exemple entre l'enseignement primaire et l'enseignement supérieur, et entre les soins médicaux primaires et les soins curatifs. Améliorer la gestion des affaires publiques au service du développement humain est un domaine où une action des pouvoirs publics peut apporter beaucoup.

\section{Sécurité humaine : réduire la vulnérabilité et gérer les crises}

L'expression sécurité humaine renvoie aux moyens de préserver le développement humain en protégeant les populations de toute privation induite par des perturbations ou des chocs extérieurs. Les pauvres, femmes et hommes, voient eux-mêmes dans l'insécurité un aspect essentiel de la pauvreté et l'une de ses causes fondamentales. Les risques auxquels sont exposés les pauvres sont considérables et ont de multiples origines, comme les pénuries de vivres, la maladie, la vieillesse, les calamités naturelles, le chômage ou d'autres aléas économiques. Ces risques appellent la mise en place de régimes de protection sociale qui doit d'ailleurs être l'un des axes prioritaires de la politique sociale. Les femmes pauvres sont particulièrement exposées au risque de violence domestique $^{21}$. Quant aux travailleurs pauvres, ils sont soumis à des risques professionnels majeurs ${ }^{22}$.

Globalement, la vulnérabilité des pauvres s'accroît à mesure que de nouvelles sources de conflits violents et de délinquance apparaissent, et qu'augmentent les risques de catastrophes naturelles consécutives au changement climatique et aux variations saisonnières extrêmes. Les inégalités entre groupes ethniques et sociaux peuvent aboutir, et aboutissent parfois, à des conflits violents, qui perpétuent et aggravent la pauvreté, et prélèvent leur tribut sur les personnes - morts, blessés, invalides et personnes déplacées - en mettant en outre à mal les infrastructures, la performance économique et le tissu social. Les conflits détruisent invariablement le capital social et exacerbent les dysfonctionnements dans la gestion des affaires publiques, parfois jusqu'à l'effondrement même de l'État ${ }^{23}$.

Toute approche fondée sur la sécurité humaine vise à répertorier les risques auxquels peuvent être exposés les pauvres, hommes et femmes, et à y remédier. Elle transcende la séparation de plus en plus artificielle entre prévention et résolution des conflits, reconstruction après un conflit, capacité de se préparer aux catastrophes naturelles et d'organiser les secours, et action en faveur d' un mode de gestion des affaires publiques fondé sur les droits. Les actions qui se concentrent sur la diminution des sources de risques (notamment des risques liés à l'environnement qu'induit la pénurie grandissante de terres fertiles, d'eau salubre et d'air propre), et sur l'aide aux populations pauvres qui doivent y faire face, prennent de plus en plus de place dans les stratégies de lutte contre la pauvreté.
... encore faut-il qu' une utilisation efficiente soit faite des deniers publics pour améliorer les services qui profitent aux pauvres.

L'insécurité est à la fois un aspect et une cause de la pauvreté ,...

... elle est liée à la délinquance, aux conflits et aux catastrophes naturelles,...

... d'où la nécessité d'une approche fondée sur la sécurité humaine. 
Une réduction durable de la pauvreté appelle une prise en compte systématique des besoins des hommes comme des femmes dans la formulation des politiques...
... et la préservation de l'environnement dans le cadre de stratégies visant à assurer la pérennité des moyens de subsistance.
Prise en compte systématique de la problématique homme-femme et promotion de l'égalité homme-femme

L'inégalité entre les hommes et les femmes est à la fois une cause essentielle de pauvreté et un obstacle majeur à l'instauration d'un développement durable. Pour réduire l'inégalité entre hommes et femmes, il convient d'améliorer l'accès des femmes à l'emploi, au crédit et à d'autres ressources productives, de façon à leur permettre de gagner un revenu. Il est apparu que c'est là un moyen de favoriser une croissance économique globale plus rapide et plus profitable aux pauvres, bénéficiant aux femmes et aux enfants aussi bien qu'aux hommes.

Les stratégies de lutte contre la pauvreté doivent viser à aplanir les disparités d'accès aux ressources et aux chances entre les hommes et les femmes. Le degré de pauvreté est conditionné non seulement par le volume et la variété des ressources matérielles et immatérielles dont dispose un ménage, mais aussi par leur répartition au sein du foyer. L'inégalité entre les hommes et les femmes n'est pas l'apanage des ménages pauvres ; elle s'observe aussi dans les structures locales et nationales.

Assurer la prise en compte systématique de ces sexospécificités dans l'élaboration des politiques peut être un axe constructif de dialogue et de coopération entre les pouvoirs publics, la société civile et les organismes de développement. En travaillant de concert, ceux-ci peuvent réduire les disparités entre les hommes et les femmes dans l'accès qui leur est donné aux services de base, aux opportunités économiques et à des actifs, comme la terre et le crédit, au marché du travail, à la vie politique, au savoir et à la technologie.

Pour être efficaces, les stratégies de lutte contre la pauvreté doivent tenir compte des relations existant entre les hommes et les femmes, et accorder une attention particulière au manque de temps dont souffrent les femmes qui cumulent un travail rémunéré et des activités domestiques non rémunérées. Les pouvoirs publics doivent prendre conscience du fait que le sexe est un motif d'exclusion dans l'élaboration de leurs cadres juridiques, institutionnels et stratégiques, par exemple leurs choix d'affectation des dépenses publiques. Dans plusieurs pays, la production vivrière, la santé et l'éducation des enfants et l'évolution démographique sont autant de domaines où des évolutions positives ont été observées depuis que les budgets nationaux tiennent compte des sexospécificités. Un suivi attentif et des évaluations rigoureuses permettront de se faire une idée plus précise de l'efficacité de ces budgets « sexospécifiques ».

Prise en compte systématique de la nécessité d'assurer la pérennité de l'environnement par l'instauration de modes durables d'utilisation des moyens d'existence

Les objectifs internationaux de développement, au nombre desquels figure la réduction de la pauvreté monétaire, le développement social et la préservation et la réhabilitation de l'environnement, sont étroitement liés à la notion politique de développement durable. C'est pourquoi il peut se révéler utile de mener une stratégie axée sur la pérennité des moyens d'existence et mettant l'accent sur le développement rural à grande échelle. Améliorer l'accès à l'éducation, à la santé, à l'eau, à la propriété foncière, à la technologie et aux services de conseil, aux moyens de communication et au crédit est une étape capitale pour renforcer la capacité des pauvres vivant en milieu rural d'accroître leurs revenus, que ceux-ci proviennent ou non de l'agriculture. Il faut avant tout s'appliquer à diversifier les moyens de subsistance et à lever les obstacles systémiques qui limitent l'aptitude des pauvres, hommes et femmes, à se sortir de la pauvreté, par exemple en améliorant la productivité agricole et la sécurité alimentaire. 
Encadré 4. Convergence des politiques intéressant la pauvreté en milieu rural, l'environnement et le développement durable

Les pauvres vivant dans des zones rurales, et notamment ceux qui ne possèdent pas de terre, dépendent totalement des écosystèmes qui les entourent (forêts, terres humides et zones de pêche côtière) pour leur approvisionnement en nourriture, combustibles, fourrage pour les bêtes et plantes médicinales. Toute dégradation de l'environnement représente donc pour eux une menace directe. Les politiques visant à faire reculer la pauvreté en milieu rural doivent impérativement s'accompagner de stratégies de développement durable cohérentes dans les domaines de l'agriculture et de l'environnement, y compris des mesures destinées à :
- Affirmer le droit des pauvres d'avoir une sécurité d'accès aux ressources productives et aux services sociaux.

- Donner la priorité aux investissements dans des modes de gestion durable des zones écologiquement fragiles.

- Encourager la diffusion de méthodes de culture adaptées, à haut rendement et écologiquement viables.

- Réorienter les ressources vers les pauvres.

- Favoriser la diversification des moyens de subsistance autres que l'agriculture dans les zones rurales.

- Associer les pauvres aux processus de prise de décision.

Par moyens d'existence, on entend les capacités ${ }^{24}$ et les activités nécessaires pour accéder à un niveau de vie adéquat dans la société. Un mode de vie est durable lorsqu'il donne la possibilité de surmonter les tensions et les crises sans porter préjudice aux ressources naturelles qui en sont la source. Les moyens d'existence à la disposition des pauvres comprennent les ressources naturelles, mais ne s'y limitent pas. Ils subissent l'influence de facteurs économiques et du mode de gestion, de chocs externes et des évolutions générales, et dépendent aussi des dotations naturelles. La reconnaissance de cet état de fait est le point de départ de toute action visant à obtenir à la fois une réduction de la pauvreté et un développement durable.

Cela implique d'admettre l'importance des liens entre les sphères micro-économique, méso-économique et macro-économique, et de privilégier les mécanismes, institutionnels et autres, qui influent sur la disponibilité et la valeur des ressources dont les pauvres, hommes ou femmes, tirent leur subsistance. Il faut tenir compte de tous les facteurs qui ont des retombées sur les moyens d'existence des différents groupes de pauvres, y compris les relations de pouvoir et de dépendance, et non s'en tenir à des hypothèses reposant sur des extrapolations ou des approches sectorielles. L'approche fondée sur la pérennité des moyens d'existence renvoie à une démarche ascendante partant des perceptions et des priorités exprimées par les pauvres eux-mêmes. Ces dernières doivent ensuite être conciliées avec les conceptions des organismes de développement et des autorités, qui appréhendent le développement durable dans une perspective plus large, nationale et internationale.

\section{Conclusion}

L'élaboration de toute stratégie digne de ce nom de lutte contre la pauvreté passe d'abord par la définition d'objectifs clairs, puis par une réflexion s'articulant autour de deux axes : l'identification des principales relations de causalité et le choix des formes d'intervention les plus efficaces. La pauvreté est pluridimensionnelle et doit être appréhendée dans toutes ses dimensions lors de la phase de planification stratégique des politiques, programmes et projets. À des fins de comparaison dans le temps et entre pays, il peut être utile de recourir à un indice composite ou à un indice quantitatif $\mathrm{du}$ revenu. 
La hiérarchisation des causes et le choix des meilleurs moyens d'action au service de la lutte contre la pauvreté sont des aspects délicats, mais essentiels. Le tableau 1 peut faciliter l'entreprise en montrant comment sélectionner et ordonner les déterminants de la pauvreté et les formes d'intervention en fonction des résultats souhaités dans un contexte particulier, au niveau mondial, (sub-)continental, national, régional ou local. Les organismes de développement et leur personnel doivent avoir accès aux enseignements généraux qui se dégagent des travaux de recherche et de l'expérience accumulée et se tenir informés des données et des analyses disponibles, ou qui pourraient le devenir, à l'échelon local. 


\section{Tábleau 1. S'attaquer à la problématique de la pauvreté : causes, action requise et résultats}

Ce tableau présente un récapitulatif des causes de la pauvreté, des actions engagées pour y remédier et des indicateurs existants pour en évaluer les résultats. Il ne doit pas nécessairement être lu comme une matrice, où les liens de causalité fonctionneraient horizontalement, de gauche à droite. Chacune des causes de la pauvreté peut nécessiter une action dans plusieurs domaines. Une action peut être liée prioritairement à un ensemble de causes et de résultats, mais influer également sur d'autres. Dans une large mesure, les causes, actions et résultats ont mutuellement des répercussions les uns sur les autres.

CAUSES :
Facteurs récurrents
Structures économiques :
rythme, configuration et qualité de
la croissance
taux d'épargne et d'investissemen
(y compris affectations sectorielles
et choix technologiques)
inflation
- systèmes incitatifs au niveau
micro-économique
- échanges (accès aux marchés
d'exportation, évolution et
instabilité des termes de
l'échange, politiques
commerciales)
inégalité d'accès aux actifs (par
sexe, groupe ou région)
inégalité d'accès aux marchés et
services
situation du marché du travail
discrimination institutionnelle à
l'encontre des femmes sur le
marché du travail et d'autres
marchés
utilisation non durable des
ressources

Accès à des ressources de qualité :

- capital naturel (eau, forêts, terre, etc.), y compris le patrimoine commun de ressources naturelles, souvent surexploité

- capital physique (animaux, outils, équipements, infrastructure)

- capital humain (santé, éducation, compétences)

- capital social (avantages de l'association)

- capital financier (épargne, crédit)

\section{ACTION REQUISE : \\ - Mesures et domaines d'action}

\section{Croissance économique fondée sur une} dynamique de réduction de la pauvreté :

- bonne gestion économique : processus d'élaboration des politiques prévisible et transparent

- politiques de redressement macro-économique

- mesures visant à infléchir la tendance à privilégier les zones urbaines/à promouvoir la croissance agricole et le développement rural

- gestion volontariste de l'intégration aux marchés extérieurs, en commençant par l'ouverture des marchés régionaux

- création et redistribution d'actifs

- mesures visant à élargir l'accès aux marchés, notamment pour les femmes, et à supprimer les distorsions sur le marché

- développement et surveillance du secteur financier, notamment réglementation prudente applicable aux flux extérieurs et accès équitable des hommes et des femmes aux ressources financières

- promotion du développement économique local, notamment micro-financement et services de conseil aux entreprises

- intégration des stratégies de croissance dans le cadre des stratégies nationales de développement durable (SNDD)

\section{Responsabilisation, renforcement des droits} et gestion des affaires publiques fondée sur une dynamique de réduction de la pauvreté :

- respect du droit dans un régime démocratique

- efforts juridiques et de sensibilisation sur les droits de l'homme

- soutien en faveur des droits des citoyens et de la société civile

- promotion des droits économiques et sociaux des pauvres et en faveur des pauvres

- renforcement des capacités pour permettre à la communauté de retrouver confiance et lui donner les moyens de revendiquer ses droits

- droit à la liberté d'association et à un travail décent
RÉSULTATS :

- Indicateurs (et sources)

\section{Capacités économiques :}

- ampleur, gravité et acuité de la pauvreté et consommation des ménages (enquêtes sur les ménages)

- portefeuilles d'actifs des plus pauvres (enquêtes sur les ménages)

- part respective des femmes et des hommes dans la consommation et dans l'utilisation du temps (études de cas approfondies)

\section{Capacités politiques :}

- auto-évaluation de l'absence de pouvoir (bilans de pauvreté participatifs)

- évaluation des relations de pouvoir au niveau local et de leur dynamique

- enquêtes sur l'équilibre entre les hommes et les femmes dans la prise de décision à tous les niveaux

- réglementations visant à décentraliser les processus de décision

\section{Capacités humaines :}

- maîtrise du développement et de la destruction de l'environnement

- mortalité infantile et juvénile

- mortalité maternelle

- prévalence du VIH/mortalité due au sida

- indicateurs de suivi des maladies au niveau local

- niveau d'instruction élémentaire

- équilibre entre les garçons et les filles au niveau scolaire 


\section{Tableau 1. S'attaquer à la problématique de la pauvreté : causes, action requise et résultats (suite)}

\section{CAUSES :}

- Facteurs récurrents

\section{Gestion des affaires publiques} et services publics :

- absence de participation de la population et de transparence dans la vie politique

- non-respect des droits de l'homme, notamment de la liberté d'association, d'expression et des médias

corruption

- inefficacité des services publics

- discrimination en fonction du statut social, du sexe et de l'appartenance ethnique dans l'accès aux services

— accès limité et piètre qualité des services rendus aux plus pauvres

- centralisation des processus de décision et des structures

\section{Configuration démographique :}

- taux de croissance démographique élevés (transition démographique différée, fécondité excessive, grossesses fréquentes)

- hausses brutales de la mortalité, notamment sous l'effet du $\mathrm{HIV} / \mathrm{sida}$

- isolement géographique

- exode rural

\section{Exclusion sociale :}

- les exclus cumulent les difficultés d'accès

- érosion ou absence pure et simple des mécanismes visant à maintenir une intégration sociale minimale des plus pauvres (personnes âgées, veuves, handicapés, populations indigènes)

- la distance et la discrimination sociale réduisent les plus pauvres au silence

- la pauvreté économique contraint les plus pauvres à recourir à des moyens d'existence incompatibles avec toute dignité sociale

- schémas culturels et juridiques patriarcaux

alcoolisme

- violence à l'encontre des femmes
ACTION REQUISE :

- Mesures et domaines d'action

\section{Services sociaux de base à l'appui du} développement humain :

- budgets nationaux et gestion des dépenses axés en priorité sur la lutte contre la pauvreté et l'égalité homme-femme

- affectation des ressources fondées sur une dynamique de réduction de la pauvreté dans les secteurs sociaux

- examen des dispositifs de financement et de l'incidence de l'impôt et des redevances d'utilisation, et de leur impact sur l'accès aux services et la qualité des services

- bonne gestion des services sociaux et incitations à améliorer ces services

\section{Prise en compte systématique de la} problématique homme-femme et action visant à promouvoir l'égalité homme-femme :

travail juridique et éducatif sur les droits des femmes, notamment droits et santé génésiques, et la violence domestique

- actions de sensibilisation à la pauvreté et à l'inégalité entre les hommes et les femmes dans des domaines tels que l'agriculture, l'éducation et la santé

- soutien à un effort de sensibilisation au manque de temps

\section{Pérennité de l'environnement grâce à des stratégies d'utilisation durable des moyens d'existence :}

- conception ascendante de l'élaboration de l'action gouvernementale, s'appuyant sur une analyse des conditions de vie des populations rurales, et en particulier de l'incidence des évolutions et chocs externes et des variations saisonnières, ainsi que sur une analyse des stratégies adoptées pour y faire face

- réforme de l'action gouvernementale, des institutions et des organisations agissant sur les moyens d'existence des pauvres qui vivent en milieu rural

- amélioration de l'accès des pauvres vivant en milieu rural aux actifs physiques, humains, financiers, naturels et sociaux

- recherche et vulgarisation fondées sur l'exploitant lui-même et privilégiant la sécurité alimentaire

- bonne gestion des services collectifs et locaux

\section{RÉSULTATS :}

- Indicateurs (et sources)

\section{Capacités sociales :}

- analyse des différents classements de la pauvreté et du bien-être au niveau local (bilans de pauvreté participatifs)

- mise en évidence des schémas d'interaction sociale par sexe, groupe ethnique et autres catégories sociales

- nombre d'organisations à l'échelon local et intensité de leur activité

\section{Capacités défensives : sécurité,} réduction de la vulnérabilité :

- fréquence et impact des conflits et des catastrophes naturelles

- mouvements de population

- auto-évaluation du bien-être (bilans de pauvreté participatifs)

- enquêtes sociales, rapports sur des sites témoins, observatoires du « climat social » 


\section{Tableau 1. S'attaquer à la problématique de la pauvreté : causes, action requise et résultats (suite)}

CAUSES :
[ Facteurs récurrents
Perturbations et conflits:
- expulsion/déplacement de
populations à cause de la guerre
conflit intra-national
- catastrophes naturelles
crises économiques
- effondrement de
l'État/désintégration du tissu
social

ACTION REQUISE :

- Mesures et domaines d'action

- surveillance active et protection des ressources naturelles dans le cadre de la stratégie nationale de développement durable (SNDD)

\section{Sécurité humaine : réduire la vulnérabilité et} gérer les chocs :

- soutien à la construction de la paix et à la reconstruction

- soutien aux réformes du secteur de la sécurité nationale dans le cadre d'un régime démocratique

- action en faveur de la cohésion sociale grâce au développement de la société civile et à la tolérance entre les cultures

- constitution d'actifs qui garantissent contre les catastrophes et les crises économiques, notamment infrastructure et assurances

- sauvegarde des systèmes de protection sociale qui fonctionnent

- action au niveau local visant à identifier les exclus de la société et les "invisibles"
RÉSULTATS :

- Indicateurs (et sources) 


\section{Notes}

1. Les notions de pauvreté et de bien-être ont valeur d'antonymes et renvoient à des situations qui se placent aux deux extrêmes de la fourchette. Les deux notions sont utilisées dans le texte, en fonction du contexte.

2. Les objectifs de développement du millénaire (voir page 19) fournissent divers exemples de mesures de la pauvreté absolue.

3. Voir les Lignes directrices du CAD pour l'égalité homme-femme et le renforcement du pouvoir des femmes dans le cadre de la coopération pour le développement (1998) et le Compendium du CAD de notions et approches relatives à l'égalité homme-femme (1998).

4. A savoir la régulation des cycles de l'eau, la production de biomasse, l'assimilation des déchets, et bien d'autres encore, qui conditionnent la pérennité des moyens d'existence générés par toute une palette d'activités économiques. Au-delà de certains seuils critiques, la dégradation des écosystèmes devient irréversible.

5. La distinction entre catastrophes «naturelles » et « induites par l'activité humaine » n'est pas toujours tranchée. Voir les Lignes directrices sur l'atténuation des effets des catastrophes naturelles, Lignes directrices du CAD sur l'aide et l'environnement, $\mathrm{n}^{\circ} .7$ (1994).

6 . Voir la note 30.

7. Les statistiques du PNUD sont pour la plupart ventilées par sexe et donc utiles comme indicateurs généraux du degré de discrimination à l'encontre des femmes et d'inégalité entre les sexes.

8. L'indicateur du développement humain (IDH), l'indice sexospécifique du développement humain (ISDH), l'indicateur de la participation des femmes (IPF) et l'indicateur de la pauvreté humaine (IPH-1 pour les pays en développement et IPH-2 pour les pays de l'OCDE et les pays en transition). Le Rapport sur le développement humain fournit aussi des indicateurs pluridimensionnels, ventilés par sexe, du développement humain.

9. La distinction est importante : l'objectif de développement du millénaire relatif à l'éradication de l'extrême pauvreté et de la faim renvoie à la proportion de personnes vivant avec moins d'un dollar par jour, laquelle a régressé aux cours des dix dernières années, tandis que le nombre de personnes vivant en dessous du seuil de pauvreté a suivi une évolution en dents de scie qui l'a laissé, en 1998, à peu près au même niveau qu'en 1987.

10. Les chapitres 2 et 5, respectivement, fournissent d'autres orientations à l'intention des organismes d'aide sur la gestion des programmes par pays d'une part, et le renforcement des capacités et les changements institutionnels, d'autre part.

11. Les domaines d'action prioritaires et les stratégies d'action gouvernementale correspondent globalement, mais pas exactement, aux dimensions de la pauvreté et aux aspects transversaux (égalité homme-femme et environnement) évoqués précédemment. Les dimensions de la pauvreté ne coïncident pas exactement avec les domaines d'action institutionnalisés et les stratégies ayant une influence sur eux. La dimension socioculturelle et l'approche axée sur la pérennité des moyens d'existence sont des aspects étroitement imbriqués et néanmoins distincts. 
12. Le taux de conversion de la croissance économique en réduction de la pauvreté monétaire varie considérablement entre les pays et entre les régions en fonction de l'ampleur des inégalités face à l'instruction élémentaire, la détention de terres et d'autres facteurs essentiels. A titre d'exemple, entre 1960 et 1994, pour un point de croissance économique par habitant, la pauvreté a diminué 4 à 5 fois plus dans certains états de l'Inde que dans d'autres, et des disparités du même ordre ont été constatées lors de comparaisons entre l'Asie de l'Est et l'Amérique latine (The Quality of Growth, Banque mondiale, 2000).

13. Des études économétriques réalisées par la Banque mondiale montrent que si la croissance du revenu moyen est le facteur le plus important pour faire reculer la pauvreté, elle n'explique toutefois qu'à peine la moitié de la croissance du revenu des pauvres. La corrélation entre la croissance et la pauvreté est significative, mais partielle ; l'inégalité des revenus est un facteur déterminant, or l'influence que la croissance exerce sur cette dernière ne va pas toujours dans le même sens ; la réalité qui se cache derrière ces moyennes statistiques est très contrastée. (David Dollar et Aart Kraay : Growth IS Good for the Poor, Banque mondiale, 2000 ; Martin Ravallion : Growth, Inequality and Poverty : Looking Beyond Averages, Banque mondiale, 2000.

14. Au Bangladesh, le pourcentage de pauvres est supérieur à $60 \%$ dans les régions où la superficie moyenne des terres détenues par un ménage est inférieure à 1 acre, mais tombe à $10 \%$ lorsque cette superficie passe à 10 acres.

15. Lucia Hanmer, John Healey, Felix Naschold : Will Growth Halve Global Poverty by 2015 ? ODI Poverty Briefing 8 juillet 2000.

16. Ce qui tient partiellement au système de classification statistique et administrative. La distinction urbain-rural n'est pas tranchée et les définitions utilisées au niveau national varient considérablement.

17. Voir La gageure de mettre fin à la pauvreté rurale, Rapport sur la pauvreté rurale 2001 du Fonds international de développement agricole (FIDA).

18. Richard Sandbrook : Citizenship, Rights and Poverty - Narrowing the Gap between Theory and Practice. Document présenté au Forum sur la démocratie organisé en 2000 par l'Institut pour la démocratie et l'assistance électorale (IDEA).

19. Essentiellement la Déclaration universelle des droits de l'homme des Nations unies (1948); deux Pactes internationaux portant respectivement sur i) les droits civils et politiques (1966) et ii) les droits économiques, sociaux et culturels (1966) ; la Convention internationale sur l'élimination de toutes les formes de discrimination raciale (1966) et la Convention internationale sur l'élimination de toutes les formes de discrimination à l'égard des femmes (1979), la Convention contre la torture et autres peines ou traitements cruels, inhumains ou dégradants (1984) et la Convention relative aux droits de l'enfant (1989) ; enfin, la Déclaration de l'OIT relative aux principes et droits fondamentaux au travail (1989).

20. Par exemple, l'Initiative 20/20, arrêtée au Sommet mondial pour le développement social organisé par les Nations unies à Copenhague en 1995, et confirmée à la Session extraordinaire de l'Assemblée générale des Nations unies à Genève en 2000, en vertu de laquelle les organismes de développement et les gouvernements partenaires s'engagent à allouer au moins $20 \%$ de leur APD bilatérale et de leur budget national, respectivement, aux services sociaux de base.

21. D'après l'OMS et l'UNIFEM, au moins $20 \%$ des femmes dans le monde ont subi des violences physiques ou sexuelles. Dans les pays en développement, ces chiffres sont nettement plus élevés ; dans certains, les deux tiers des femmes vivant en milieu rural et plus de la moitié des femmes vivant en milieu urbain sont victimes de violences physiques ou sexuelles. La Banque mondiale estime qu'à l'échelle de la planète, la violence à l'encontre des femmes est une cause de décès et d'invalidité aussi fréquente que le cancer parmi les femmes en âge de procréer, et une cause de mauvaise santé plus répandue que les accidents de la circulation et le paludisme réunis. 
22. L'OIT estime à 250 millions par an le nombre d'accidents du travail et à 160 millions par an le nombre de victimes de maladies professionnelles. Dans les pays en développement, où un grand nombre de travailleurs sont employés dans le secteur primaire et les industries extractives, notamment dans l'agriculture, l'exploitation forestière, la pêche et les mines, qui figurent parmi les activités les plus dangereuses dans le monde, le bilan est particulièrement lourd en nombre de morts et de blessés.

23. Voir les Lignes directrices sur les conflits, la paix et la coopération pour le développement à l'aube du XXI ${ }^{e}$ siècle, Série des Lignes directrices sur la coopération pour le développement, CAD/OCDE, 1998, et son complément intitulé Contribuer à prévenir les conflits violents : orientations à l'intention des partenaires extérieurs, CAD/OCDE, 2001.

24. Capacités économiques, humaines, politiques, socioculturelles et défensives, voir la section intitulée Les principales dimensions de la pauvreté. 


\section{2 Édifier des partenariats pour lutter contre la pauvreté}

\section{Introduction}

L'efficacité de la lutte contre la pauvreté dépend souvent des activités concrètes menées à l'échelon des pays et de leurs modalités de mise en œuvre. De tout temps, les organismes de développement ont eu des difficultés à concrétiser leurs politiques en la matière. Le chapitre 2 a pour objet de montrer comment ils peuvent œuvrer en coopération avec les pays en développement à l'édification de solides partenariats et à la traduction de la politique définie en des programmes et des interventions plus efficaces sur le terrain.

On commencera ici par exposer les grands principes devant sous-tendre les changements destinés à améliorer les performances des organismes de développement en tant que partenaires dans la lutte contre la pauvreté. On se concentrera ensuite sur les bonnes pratiques à respecter pour construire des partenariats, faire face à la diversité des situations des pays partenaires et assurer une interaction efficace avec d'autres partenaires. La conclusion récapitule les domaines sur lesquels devra porter en priorité l'action des organismes de développement pour réellement donner corps sur le terrain à des partenariats au service de la lutte contre la pauvreté.

\section{Principes fondamentaux de tout partenariat au service de la lutte contre la pauvreté}

Les principes fondamentaux qui doivent régir la coopération entre les organismes de développement et leurs partenaires au service de la lutte contre la pauvreté sont les suivants :

L'appropriation nationale, régionale et locale des stratégies, politiques et priorités de développement est essentielle. Les stratégies et programmes de lutte contre la pauvreté ont un but politique dans la mesure où ils visent à offrir des opportunités à certains groupes de la société et à leur affecter des ressources. Aucun progrès n'est possible si les principales parties prenantes aux niveaux national et local ne souscrivent pas aux réformes économiques et politiques nécessaires. C'est pourquoi il est primordial que l'ensemble des acteurs du pays partenaire, au niveau national, régional et local, s'approprient les mesures et programmes en faveur des pauvres. Cela dit, la qualité de la gestion des affaires publiques, en particulier les efforts des pouvoirs publics pour donner aux stratégies menées une orientation favorable aux pauvres, demeure un critère déterminant du soutien des organismes de développement.

Le partenariat est capital pour faciliter l'appropriation locale. Pour réussir, les partenariats pour le développement doivent respecter certaines conditions et modalités qui peuvent remettre en question les méthodes traditionnelles de certains organismes de développement (voir ci-après "les défis du partenariat"). Les partenariats les plus efficaces se sont révélés être ceux qui reposent sur une définition claire des droits et devoirs de chaque partenaire et sur un accord sur les objectifs visés et les moyens de les atteindre. Les processus d'élaboration, à l'échelon local, de stratégies de lutte contre la pauvreté portent en eux la promesse de devenir la pierre angulaire de la constitution, au niveau de chaque pays, de solides partenariats au service de la lutte contre la pauvreté (voir chapitre 3 "cadres stratégiques qui se mettent en place").
Le succès des efforts de lutte contre la pauvreté est souvent conditionné par la nature et les modalités de mise en œuvre des activités entreprises au niveau de chaque pays.

Parmi les principes fondamentaux devant sous-tendre les efforts de lutte contre la pauvreté déployés par les organismes d'aide figurent désormais I'instauration de partenariats de nature à favoriser l'appropriation locale,... 


\section{Encadré 5. Recommandations pratiques pour un bon fonctionnement des partenariats au service de la lutte contre la pauvreté}

Travailler en partenariat suppose que les organismes de développement changent radicalement leurs modes de relation avec les autres partenaires (gouvernement, société civile) et entre eux (institutions multilatérales, autres organismes bilatéraux). Cela suppose aussi qu'ils changent profondément leur manière de travailler dans les pays partenaires. On trouvera ci-dessous des recommandations pratiques destinées à améliorer le fonctionnement des partenariats.

- Prendre comme cadre général de la coopération pour le développement la stratégie de lutte contre la pauvreté et le budget national du pays partenaire.

- Reconnaître que c'est au gouvernement du pays partenaire qu'il revient d'assumer la direction des opérations.

- Définir clairement les rôles et responsabilités des différents acteurs (gouvernement, organismes bilatéraux, institutions financières internationales et régionales, organismes des Nations unies, société civile, syndicats, secteur privé).

- Ne jamais travailler seul. Avant d'entamer une discussion ou une action, rechercher d'autres partenaires (dans la communauté de l'aide au développement, le gouvernement ou la société civile) susceptibles d'y participer.

- Investir dans des mécanismes de coordination (qui doivent permettre, sous la direction du pays, de coordonner la planification stratégique ex ante et la mise en œuvre conjointe), et définir de façon précise les modalités, le moment et le lieu des échanges avec les autres partenaires extérieurs et locaux.

- Promouvoir et renforcer les travaux communs (collecte de données, analyses, missions, évaluations, gestion et comptabilité des apports d'aide) et partager l'information (données, analyses, actions et programmes envisagés) avec les autres partenaires.

- Simplifier et rationaliser, dans la mesure du possible, les exigences administratives et financières de l'organisme de développement (par exemple en ce qui concerne la gestion financière et la reddition de comptes, les phases préparatoires du cycle des projets, la notification et le suivi) et renforcer les systèmes mis en place par l'administration du pays partenaire dans ces mêmes domaines.

- Faciliter la mobilisation, la participation, la surveillance et l'évaluation locales.

- Euvrer au renforcement des capacités afin d'affermir la prise en main par le gouvernement de la coordination de la lutte contre la pauvreté et des processus de consultation, et de permettre à la société civile, y compris aux organisations féminines et à celles qui militent en faveur de l'égalité des sexes, de participer pleinement au processus de consultation et de suivre activement et d'évaluer les politiques et programmes de lutte contre la pauvreté - sans pour autant porter préjudice à l' autorité du gouvernement partenaire ou aux institutions démocratiques nationales.

\section{... l'évaluation de I'adéquation des stratégies locales, la promotion de la participation locale et Il'émancipation des femmes,...}

\section{... l'établissement de relations à long terme...}

Une bonne évaluation du contexte local est fondamentale. La stratégie de lutte contre la pauvreté définie au niveau local peut ne pas correspondre à ce que les organismes de développement considèrent comme la meilleure solution sur le plan de la portée, des priorités et du calendrier de l'action envisagée. Le soutien à apporter à la stratégie du pays partenaire devra s'appuyer sur un bilan des avantages, des inconvénients et des compromis qu'implique l'approche retenue compte tenu de la marge de manœuvre qu'autorise le contexte économique, politique et social local.

Il faut mettre l'accent sur la participation et la démarginalisation. La participation à tous les niveaux et à toutes les phases de la coopération pour le développement est indispensable si l'on veut améliorer les politiques en faveur des pauvres, la transparence de leur mise en œuvre, et le caractère durable des résultats grâce à l'appropriation à l'échelon local. Donner des moyens d'action aux individus, aux familles et aux communautés est essentiel pour valoriser le capital humain et permettre aux pauvres de s'intégrer dans la vie sociale, politique et économique de leur pays et de façonner leur propre avenir. Il est de plus en plus largement admis que la démarginalisation des pauvres est capitale pour que la lutte contre la pauvreté produise des résultats.

La réduction de la pauvreté passe par l'émancipation des femmes. L'expérience a montré l'énorme impact que peuvent avoir sur la réduction de la pauvreté des activités visant à promouvoir les droits des femmes, à leur offrir des chances et à favoriser leur émancipation. C'est pourquoi toute politique de coopération pour le développement centrée sur la pauvreté doit prendre 
en compte la problématique homme-femme. Une coopération soucieuse d'équité entre les sexes a des répercussions directes en termes d'amélioration des moyens d'existence et d'atténuation des discriminations, et se révèle particulièrement avantageuse sur les plans de la croissance, de l'alphabétisation, de la nutrition des enfants, etc.

Coordination et engagement durable sont des conditions importantes lorsqu' on veut construire des partenariats et faire reculer la pauvreté. Il n'y a pas de solutions rapides pour améliorer le bien-être social, politique et économique des pauvres. Une action au coup par coup est considérée comme particulièrement préjudiciable aussi bien à l'efficacité de l'aide qu'à la réduction de la pauvreté. Les bonnes pratiques impliquent au contraire un engagement à long terme dans un cadre clairement défini d'un commun accord.

Les pratiques en matière de développement et les résultats aux quels elles aboutissent doivent faire l'objet d'un suivi et d'évaluations qui permettront de mesurer les avancées obtenues grâce au partenariat et de $s^{\prime}$ assurer que les effets produits sont et restent bénéfiques pour les pauvres. Le suivi est nécessaire, non seulement pour s'assurer que l'on atteint les objectifs souhaités en matière de réduction de la pauvreté, mais aussi pour voir si les actions en faveur du développement ont des effets secondaires préjudiciables aux pauvres. Quant à l'évaluation, elle est indispensable pour déterminer l'impact, mesurer les progrès et tirer des enseignements de l'expérience. Le suivi et l'évaluation doivent être assurés en partenariat avec les pouvoirs publics, les acteurs locaux et les autres organismes extérieurs.

\section{Les défis du partenariat}

\section{Que faut-il pour instaurer de bons partenariats au service de la lutte contre la pauvreté ?}

Un partenariat solide et productif entre un gouvernement, la société civile et la communauté du développement se fonde sur la confiance, la transparence, et un même engagement sur les objectifs et les résultats à atteindre. C'est lorsqu'il se fonde sur des relations réciproques caractérisées par une définition claire des rôles et responsabilités de chacun et par un dialogue ouvert et sans exclusive qu'il est le plus efficace. Les critères essentiels de cette efficacité sont présentés dans l'encadré 5.

Travailler en partenariat implique plusieurs défis :

Les questions auxquelles les mandants des organismes de développement attachent une grande importance (l'environnement, l'égalité entre les sexes, la bonne gestion des affaires publiques) doivent être intégrées dans le dialogue sur les politiques à suivre sans pour autant qu'un programme d'action soit imposé de l'extérieur.

- Il importe de favoriser entre l'administration centrale et les autres composantes de la société (par exemple les collectivités locales, le secteur privé, les organisations de la société civile, les associations féminines et les ONG qui œuvrent en faveur des femmes et, dans la mesure du possible, les pauvres et les organisations qui les représentent) un dialogue véritable sur les stratégies locales de lutte contre la pauvreté, sans porter atteinte à la légitimité des gouvernements partenaires, ni empiéter sur le rôle du parlement et des autres institutions démocratiques clés.

- "L'efficacité du point de vue du développement » accroît la nécessité d'une approche plus sélective et plus stratégique des décisions relatives à l'affectation de l'aide, tenant compte de critères objectifs et des performances démontrées du partenaire et s'inscrivant dans une perspective à long terme.
... ainsi que

l'évaluation des

performances au

regard des objectifs à

atteindre et une large diffusion des pratiques optimales.
Se conduire en partenaire n'est pas toujours facile; cela suppose, entre autres,... 
Apprécier la qualité de la détermination d'un pays à lutter contre la pauvreté implique des jugements qualitatifs délicats sur le rythme et la portée des changements observés et sur la mise en œuvre des mesures annoncées. Les réponses aux questions suivantes pourront être utiles pour évaluer l'ampleur et la qualité de l'engagement des pouvoirs publics et déterminer dans quels domaines des progrès sont nécessaires ou un soutien s'impose :

- Une stratégie de lutte contre la pauvreté a-t-elle été définie et un plan d'action à moyen terme établi de façon participative est-il en cours d'application? Le parlement (ou une autre instance élue) et ses commissions jouent-ils un rôle important dans l'élaboration de la stratégie ?

- Quelle est la représentativité du processus de consultation des acteurs extérieurs au gouvernement (organisations non gouvernementales, régions, secteur privé, presse, groupes sociaux, y compris les organisations de femmes, par exemple) ?

- Constate-t-on une volonté de veiller à ce que les mesures de lutte contre la pauvreté donnent aux femmes pauvres davantage d'autonomie et d'opportunités ?
- Les plans et les budgets sont-ils transparents, et intégralement publiés en temps voulu?

- Y a-t-il une volonté claire et profonde de consacrer des moyens à la lutte contre la pauvreté et cela se traduit-il dans la répartition inter et intra-sectorielle des ressources?

- Les critères de réduction de la pauvreté ont-ils un poids suffisant dans les procédures d'examen des décisions relatives aux dépenses d'investissement et de fonctionnement du pays ?

- Quelles sont les étapes définies d'un commun accord qui ont été franchies par le gouvernement dans l'application de sa stratégie nationale de réduction de la pauvreté ?

- La politique définie fait-elle la part des besoins spécifiques de groupes socialement ou géographiquement défavorisés ? Prévoit-elle des mesures particulières pour atténuer les obstacles auxquels ces groupes sont confrontés ?

- Comment les différentes administrations sont-elles incitées à prendre en compte les critères de pauvreté et à y répondre par une amélioration des services de base ? Comment les fonctionnaires sont-ils incités à aller travailler dans les zones rurales, surtout lorsqu'elles sont enclavées? ....de ne pas imposer son point de vue ou ses priorités, de promouvoir un dialogue ouvert sans porter préjudice aux structures de gestion des affaires publiques et de concilier le souci d'efficacité de l'aide avec les besoins et les performances des autres.
La réciprocité est un élément déterminant du travail en partenariat. Il faut que les organismes de développement décident collectivement et en collaboration avec les pays en développement partenaires comment ils vont évaluer mutuellement leurs choix politiques, leurs programmes de dépenses et leurs contributions aux résultats obtenus dans le domaine de la lutte contre la pauvreté. Ce type de réciprocité renforce la confiance et la détermination des autres partenaires. Il faudra pour cela étendre progressivement l'application des indicateurs de performance à tous les partenaires, et les organismes de développement - comme les gouvernements - seront comptables de la fiabilité de leurs engagements et de la cohérence de leurs politiques.

\section{Mesure de la détermination du pays partenaire et des résultats obtenus grâce au partenariat}

Critères de mesure de la détermination du pays partenaire à lutter contre la pauvreté. La qualité du partenariat va dépendre de la qualité de la gestion des affaires publiques, et en particulier des efforts déployés par le gouvernement pour orienter ses stratégies dans un sens favorable aux pauvres et prenant en compte le problème de l'équité entre les sexes. La mesure de la détermination des pouvoirs publics en la matière et de la qualité de leur action doit s'adapter au contexte spécifique de chaque pays (encadré 6). Les critères appliqués pour évaluer la performance des partenaires devront être définis dès le début du processus d'élaboration par l'organisme de développement de son programme pour le pays considéré.

La qualité du dialogue sur les politiques à suivre et des processus de consultation est aussi un critère qui a son importance. En règle générale, la transparence vis-à-vis des parties prenantes locales, notamment des élus, est capitale. Bien qu'en pratique les parlements ou autres instances législatives nationales et les assemblées locales aient souvent des pouvoirs très limités, leur existence même constitue un frein potentiel aux 


\section{Encadré 7. Évaluer l'action des organismes de développement en matière} de réduction de la pauvreté

Travailler en partenariat implique d'accorder une grande attention à l'évaluation des performances des organismes de développement par rapport aux responsabilités et obligations définies d'un commun accord. A titre indicatif, il pourra être utile de se poser les questions suivantes :

- La stratégie par pays de l'organisme de développement est-elle fondée sur le diagnostic posé par le pays partenaire et sur sa stratégie de lutte contre la pauvreté ?

- Dans quelle mesure la stratégie par pays de l'organisme de développement prend-elle en compte le caractère pluridimensionnel de la pauvreté ?

- Dans quelle mesure les activités de coopération de l'organisme de développement (missions, évaluations préalables, collectes de données, analyses, etc.) ont-elles été menées conjointement ou en coopération avec d'autres organismes de développement bilatéraux ou multilatéraux ?

- Dans quelle mesure les exigences administratives et financières de l'organisme de développement ont-elles été harmonisées ou mises en conformité avec les procédures en vigueur dans le pays partenaire ou avec celles des autres partenaires extérieurs, pour autant que le lui permettent les impératifs qu'il doit lui-même respecter?

- Dans quelle mesure le soutien apporté par l'organisme de développement respecte-t-il et favorise-t-il l'appropriation par le pays partenaire?
- L'organisme de développement a-t-il appuyé la mise en place ou le renforcement de processus de planification, d'exécution et de coordination conduits par le pays partenaire?

- L'organisme de développement a-t-il contribué à faciliter la participation de la société civile (au niveau local, national et international) au débat sur la stratégie du pays en matière de lutte contre la pauvreté et à la définition de son contenu, dans le respect des préoccupations et de l'action du gouvernement?

- Un engagement clair et résolu a-t-il été exprimé de mobiliser des moyens au service de la réduction de la pauvreté ?

- Y a-t-il eu un engagement à fournir des ressources selon un calendrier précis à moyen terme ?

- A-t-on suffisamment veillé à éviter les doubles emplois et à tirer parti des complémentarités entre les organismes de développement extérieurs?

- Des efforts ont-ils été déployés pour améliorer la cohérence des politiques au sein de l'organisme de développement, et plus généralement entre les différents ministères et administrations, et des progrès ont-ils été réalisés dans ce sens ? détournements de fonds publics caractérisés et une incitation à privilégier les dépenses publiques qui concrétisent l'engagement des pouvoirs publics à lutter contre la pauvreté. Le dialogue entre le gouvernement et les organismes de développement ne doit pas empiéter sur le rôle du parlement mais au contraire viser à renforcer le débat et le dialogue au niveau local.

Pour juger de la continuité de l'engagement du partenaire à l'égard de la lutte contre la pauvreté, il faut pouvoir opérer un suivi des progrès accomplis dans l'application des mesures en faveur des pauvres. Cela impliquera de définir d'un commun accord des critères de performance fondés sur des données de référence et des indicateurs quantitatifs. Ceux-ci doivent permettre de mesurer l'avancement de la mise en œuvre de la stratégie de lutte contre la pauvreté, et en particulier des réformes économiques conduites par le pays partenaire pour créer un environnement propice à la croissance et favorable aux pauvres.

Critères d'évaluation de la performance des organismes de développement en matière de lutte contre la pauvreté. Les organismes de développement doivent aussi définir, en coopération avec leurs partenaires, des critères d'évaluation de leurs propres performances dans les partenariats au service de la lutte contre la pauvreté.

Le succès de la lutte contre la pauvreté dépend de nombreux facteurs, dont la plupart ne sont pas du ressort des organismes bilatéraux, ni de l'aide au développement en général. L'impact de leurs stratégies par pays sur la réalisation des objectifs de développement sectoriels et sur l'amélioration des indicateurs de la pauvreté énoncés

\author{
Il faut évaluer en \\ permanence la \\ performance des \\ pays partenaires au \\ regard des objectifs \\ de partenariat : mise \\ en œuvre de \\ politiques favorables \\ aux pauvres et \\ soucieuses des \\ disparités entre \\ hommes et femmes, \\ et promotion d'un \\ dialogue ouvert à \\ tous les segments de \\ la société, \\ notamment.
}


La performance des organismes d'aide, y compris l'intensité des efforts qu'ils déploient pour réduire la pauvreté, pour simplifier leurs exigences administratives et pour gérer le processus d'aide de manière souple et rationnelle, doit elle aussi faire l'objet d'une évaluation. dans les stratégies de lutte contre la pauvreté des pays partenaires n'en constitue pas moins un critère essentiel d'évaluation de la performance des organismes de développement. Il est donc essentiel de mettre au point des méthodes appropriées de mesure de l'impact en termes de réduction de la pauvreté.

D'autres critères peuvent venir s'y ajouter : par exemple la mesure dans laquelle l'organisme de développement coordonne, avec les autres partenaires, ses activités de planification et de mise en œuvre, s'applique à réduire la charge administrative qu'il leur impose et facilite la collaboration entre eux (encadré 7). On peut aussi envisager d'utiliser comme mesure de la performance les efforts faits pour accroître la flexibilité et la prévisibilité des moyens mis à disposition - tout en reconnaissant les contraintes auxquelles sont soumis certains organismes pour l'aide en nature.

Les organismes de développement doivent par ailleurs s'interroger sur la mesure dans laquelle les projets qu'ils soutiennent sont parfaitement cohérents avec la stratégie de réduction de la pauvreté du pays partenaire et totalement intégrés à son cadre de dépenses.

L'harmonisation des procédures qui forment le canevas du système de partenariat et une meilleure coordination de l'aide sont des ingrédients importants pour accroître l'efficacité de l'aide au service de la réduction de la pauvreté ; les efforts entrepris dans ce sens doivent donner lieu à évaluation. Chaque pays Membre possède certes des avantages comparatifs et est soumis à des contraintes politiques qui lui sont propres, de sorte qu'une certaine souplesse est nécessaire ; cela étant, il est néanmoins important de simplifier et d'harmoniser les exigences des Membres en matière de gestion financière et de reddition de comptes. Une simplification et une rationalisation de la phase précédant la mise en œuvre des projets, et des procédures de notification et de suivi s'imposent également.

Le gouvernement de chaque pays Membre du CAD doit être tenu de rendre des comptes sur l'efficacité de son aide (par exemple des mesures prises pour réduire les coûts de transaction, accroître la proportion des marchés passés localement, avancer sur la voie du déliement de l'aide, faire davantage appel à des experts locaux) et sur sa performance sur le plan du renforcement de la cohérence des politiques (en particulier des politiques de coopération pour le développement et des politiques commerciales) et de l'amélioration de l'accès à l'information et aux technologies (chapitre 4).

\section{Approches stratégiques de la répartition d'ensemble de I'aide au développement et de sa programmation}

\section{Nécessité d'affecter davantage de ressources aux pays les plus pauvres et aux plus pauvres dans les autres pays en développement}

Affecter les ressources de façon à assurer une réduction effective de la pauvreté. Étant donné le caractère limité des ressources allouées à l'aide au développement et l'importance que revêt la réduction de la pauvreté, il est capital que ces ressources soient utilisées aussi efficacement que possible. Les critères gouvernant la répartition de l'aide entre les différents pays doivent tenir compte à la fois du nombre et de la proportion de personnes très pauvres et de l'efficacité avec laquelle l'aide devrait pouvoir être utilisée dans les pays partenaires auxquels on envisage d'apporter un soutien. Pour optimiser l'impact de la coopération pour le développement en termes de réduction de la pauvreté, il faut : 
- Se concentrer sur les pays les plus pauvres, tout en continuant d'offrir des financements mesurés et ciblés à d'autres pays en développement où la pauvreté est courante.

- Mettre l'accent sur les grands pays et les pays de taille moyenne, où vit la grande majorité des très pauvres, même si le montant de l'aide par habitant reste sensiblement plus élevé dans les petits pays.

- Tenir compte des enseignements passés concernant l'efficacité de l'aide, qui ont mis en évidence l'importance de la volonté politique de combattre la pauvreté et d'un environnement qui contribue à l'efficacité des politiques et des institutions.

- S'assurer que la stratégie de lutte contre la pauvreté du pays partenaire suscite une large adhésion et est adaptée et opportune.

La pauvreté demeure un grave problème dans les pays à revenu intermédiaire, et la coopération pour le développement peut jouer un rôle moteur à l'appui de la mise en place des infrastructures matérielles et institutionnelles nécessaires et de la mobilisation de financements complémentaires en faveur du développement de services sociaux fondamentaux et d'une croissance tirée par le secteur privé.

\section{Soutien à des stratégies nationales pertinentes de lutte contre la pauvreté}

D'une façon générale, c'est la stratégie du pays partenaire qui déterminera la nature et l'importance du soutien apporté par l'organisme de développement. Chaque pays a, en matière de lutte contre la pauvreté, des besoins et des capacités qui lui sont propres ; chacun a sa conception des mesures qui s'imposent et des priorités à respecter. Aux organismes de développement d'adapter leur soutien en conséquence. La légitimité, l'adéquation et la pertinence des stratégies de lutte contre la pauvreté des pays partenaires sont des critères importants que l'organisme de développement devra prendre en compte dans sa planification et ses décisions en matière d'affectation de l'aide. Dans certains cas, l'efficacité avec laquelle le gouvernement a, par le passé, donné suite aux mesures annoncées aura aussi son importance.

\section{Le rôle de la coopération pour le développement selon le contexte national}

Les organismes de développement doivent adapter leurs stratégies par pays à la situation particulière de chaque pays partenaire, notamment aux conditions sociales et politiques, aux atouts et aux capacités des institutions nationales, à l'ampleur et à la gravité de la pauvreté et à ses caractéristiques géographiques et spatiales.

Les pays partenaires n'ont pas tous le même souci ni les mêmes possibilités de lutter contre la pauvreté ; la situation change d'ailleurs continuellement, de sorte qu'il est difficile de généraliser, d'établir des catégories et de déterminer par avance exactement ce qu'il y a lieu de faire selon le cas. Il n'est toutefois pas inutile de donner un aperçu général des différents contextes et options possibles, une sorte de «typologie notionnelle des pays partenaires », afin d'aider le personnel des organismes de développement à comprendre le rôle que peut jouer la coopération pour le développement dans un contexte national donné. D’une façon générale, malgré leurs différences, qui sont considérables, les pays partenaires peuvent se classer dans l'une des catégories suivantes :

Grands pays non tributaires de l'aide. Le rôle revenant à la coopération pour le développement dans la lutte contre la pauvreté dans ces pays est vraisemblablement marginal, compte tenu du volume de leurs équilibres macro-économiques, de leur facilité d'accès aux marchés internationaux des capitaux et de la fongibilité des flux financiers. Cela n'empêche pas qu'il faut prendre en compte les besoins des pauvres - qui peuvent
La répartition des ressources doit être opérée en fonction de la réponse à la question suivante : où l'aide peut-elle être utilisée le plus efficacement au service de la lutte contre la pauvreté ?

Une «typologie des pays partenaires » a été établi afin d'aider le personnel des organismes d'aide à déterminer les options stratégiques et les formes de soutien les plus appropriées face à telle ou telle situation. 
Lorsqu' un pays partenaire n'est pas encore parvenu au terme de sa réflexion et de ses consultations sur une stratégie de lutte contre la pauvreté, des évaluations intermédiaires des progrès réalisés sont importantes pour apprécier leur degré d'attachement à la réduction de la pauvreté. être considérables dans certains pays - et y répondre. Une priorité majeure de l'aide au développement sera de mobiliser davantage de ressources et d'actions au niveau local au profit de la lutte contre la pauvreté. L'impact de la coopération pour le développement étant moins grand dans ces pays, les efforts visant à susciter un engagement plus fort et le déploiement de moyens plus importants au service de la lutte contre la pauvreté devront privilégier le dialogue avec les partenaires, les actions de sensibilisation ainsi que les initiatives visant à renforcer la participation de la société civile à l'élaboration des politiques. Dans les pays à structure fédérale, cela peut éventuellement se faire au niveau infranational. L'aide pourra se focaliser sur des actions ciblées de développement du secteur privé (par exemple l'octroi de micro-crédits et la formation à la gestion d'entreprise, en particulier pour les femmes), l'amélioration des performances du secteur public, des structures de gouvernance et des institutions (y compris au niveau infranational) et la promotion de la cohérence des politiques des pays Membres (en particulier les politiques commerciales, agricoles et financières). L'aide au développement peut également servir à mettre au point et expérimenter des stratégies nouvelles et novatrices de lutte contre la pauvreté.

Pays qui ont élaboré une stratégie de lutte contre la pauvreté mais n'ont pas les capacités suffisantes pour la mettre en œuvre. Dans les pays où les mécanismes publics fonctionnent raisonnablement bien et où l'on fait des efforts pour remédier aux dysfonctionnements qui peuvent subsister, les stratégies et programmes des organismes de développement doivent s'intégrer dans la stratégie et les priorités d'investissement public définies par le pays en question. Il importera de se mettre d'accord sur la manière de rendre compte des résultats obtenus grâce aux financements externes sans bouleverser les priorités nationales en matière de dépenses. Dans la programmation de leur soutien aux pays en transition économique, les organismes de développement doivent tenir compte des délais nécessaires à la concrétisation des changements économiques et sociaux. Les principaux domaines où il convient $d$ 'apporter une aide à ces pays sont le développement institutionnel du secteur public (au niveau national, régional et local), le renforcement des capacités de la société civile et la coopération technique en faveur du développement sectoriel.

Pays qui s'efforcent d'élaborer des stratégies économiques et des politiques sociales pour faire reculer la pauvreté et ne disposent pas des capacités et des mécanismes institutionnels voulus. Dans les pays qui s'efforcent encore de définir une stratégie cohérente de lutte contre la pauvreté, les indicateurs de processus et les mesures intermédiaires des progrès réalisés vers les objectifs fixés sont particulièrement importants. Les indicateurs de processus spécifiques au contexte - permettant notamment de savoir si et comment les représentants des groupes pauvres ou vulnérables ont été en situation de participer au processus - peuvent aider à évaluer l'engagement à lutter contre la pauvreté. Les programmes des organismes de développement dans ces pays doivent encourager la participation de la population à l'analyse de la pauvreté. Les Membres doivent œuvrer au renforcement des capacités de ces pays d'analyser et d'interpréter les données, en particulier les données sociales, économiques et politiques qui se rapportent à l'inégalité entre les sexes et aux autres formes de marginalisation et d'exclusion sociale. Si un pays qui a la volonté de lutter contre la pauvreté n'est pas en mesure de formuler une politique, l'aide visant à renforcer les capacités d'analyse devra être complétée par des transferts de ressources prenant la forme d'un soutien à des projets conduits par le pays. On pourra également envisager de soutenir des approches sectorielles dans certains domaines et d'accorder un allégement de dette, ou des ressources en devises ou des fonds destinés à être investis. Les organismes de développement peuvent également apporter leur concours à des actions de sensibilisation au problème de la pauvreté et au dialogue sur les mesures à prendre dans ce domaine. 
Pays qui ont été victimes d'un conflit ou d'une catastrophe naturelle. Dans les pays qui sortent d'un conflit ou d'une catastrophe naturelle, les organismes de développement peuvent centrer leur aide sur la restauration des infrastructures économiques endommagées et des services de base, afin d'atténuer les retombées de ces calamités sur les pauvres. Dans ce type de situation, la réduction des risques, de la vulnérabilité et de l'insécurité qui s'attachent à la situation de pauvreté peut être intégrée aux secours d'urgence et aux efforts de remise en état ou de construction de la paix. Dans le même temps, si ces pays n'ont pas jusque-là fait la preuve d'un engagement crédible à lutter contre la pauvreté, l'aide au développement (en particulier pour le renforcement des capacités) peut se révéler décisive pour créer un espace qui permettra à leurs gouvernements de commencer à se préoccuper des impératifs de réduction de la pauvreté. Les organismes de développement doivent également envisager d'aider les médias et la société civile à constituer des alliances contre la pauvreté (notamment dans les pays qui sortent d'un conflit) et de soutenir des projets locaux visant à améliorer la gestion des affaires publiques.

\section{Pays où le gouvernement ne manifeste pas une volonté suffisante de lutter contre la pauvreté.} Dans les pays où la gestion des affaires publiques laisse à désirer, où sévit un conflit ou dont le gouvernement n'affiche aucune volonté de lutter contre la pauvreté, les types de soutien que peuvent apporter les organismes de développement sont parfois très limités. L'existence de sérieux doutes quant à la qualité de la gestion des affaires publiques, au respect des droits de l'homme et à l'efficacité de l'aide empêcheront la coopération intergouvernementale. Les organismes de développement ne peuvent alors travailler qu'avec les autorités locales ou les organisations non gouvernementales pour essayer de réduire la pauvreté parmi certaines populations ciblées. Ils peuvent aussi fournir un soutien plus général pour réduire la vulnérabilité et satisfaire les besoins humanitaires. Si l'appareil gouvernemental est inexistant ou défaillant, une action purement locale sera peut-être la seule réponse possible. Enfin, les organismes de développement bilatéraux pourraient s'interroger sur la manière dont un soutien aux structures locales, à la société civile et au secteur privé est susceptible d'ouvrir la voie à un engagement plus sérieux du pays partenaire à l'égard de la lutte contre la pauvreté, et par ricochet à une aide plus active de leur part.

Les organismes de développement doivent être conscients de l'importance qu'il y a, quel que soit le contexte national, à entretenir et mettre à profit les relations avec la société civile. C'est parce qu'ils auront su nouer des rapports étroits avec la société civile qu'ils seront à même de recueillir un retour d'information essentiel et de se faire ainsi une meilleure idée des ramifications politiques de la réduction de la pauvreté avec les intérêts des élites et des dirigeants locaux, ainsi que des comptes que les pouvoirs publics estiment avoir à rendre aux pauvres et des relations qu'ils sont prêts à nouer avec eux.

\section{Conduite à tenir en cas de problèmes}

Aider les partenaires en proie à de graves difficultés. L'enveloppe allouée à l'aide est aussi mise au service d'objectifs de développement autres que la réduction de la pauvreté, comme la prévention des conflits, les droits de l'homme et la démocratie participative, l'égalité homme-femme et le développement durable. Souvent, il arrive aussi que des pays aient un besoin aigu de soutien pour s'adapter à des chocs extérieurs, par exemple un afflux de réfugiés fuyant un conflit dans un pays voisin, une catastrophe naturelle, ou une détérioration brutale des termes de l'échange - autant d'événements qui affectent le développement économique et social.
Les possibilités s'offrant aux organismes d'aide sont limitées dans les pays où sévit un conflit, où la gestion des affaires publiques laisse à désirer ou qui ne manifestent pas une volonté réelle de faire reculer la pauvreté. 
Que doivent faire les organismes bilatéraux lorsqu' un pays partenaire ne remplit pas, ou ne remplit que partiellement, les engagements ou les responsabilités qu'il a souscrits ?

Les pays partenaires dont les performances sont satisfaisantes peuvent escompter recevoir une aideprogramme accrue tandis que ceux dont les performances restent médiocres doivent s'attendre à ce que l' aide qui leur est fournie soit davantage circonscrite - ce qui aura forcément des répercussions sur son volume.
Collaborer étroitement avec d'autres partenaires extérieurs pour faire face aux situations critiques. Les pays dont les politiques de développement et les institutions sont inadéquates ont besoin d'une aide pour mettre en place les conditions indispensables à l'amélioration de leurs performances. Travailler en partenariat avec ces pays selon des modalités favorisant l'appropriation, et garantissant néanmoins l'efficacité de l'aide et sa contribution à la réduction de la pauvreté, risque de se révéler une entreprise difficile. Que se passet-il lorsque le gouvernement d'un pays partenaire n'honore pas, ou n'honore que partiellement, les intentions qu'il a affichées ou les engagements qu'il a pris ? La fixation de conditions relatives à l'action des pouvoirs publics, souvent couplée à un soutien financier et technique, a quelquefois aidé des gouvernements (généralement au pouvoir depuis peu de temps) ouverts à la réforme à faire avancer leur programme. Mais, la conditionnalité n'a généralement été dans le passé ni efficace, ni viable, ni propre à susciter une appropriation par le pays partenaire, et ce genre de démarche n'a guère de chance de réussir dans des pays où les conditions préalables au partenariat ne sont pas réunies. Des pratiques prometteuses qui commencent à voir le jour, on peut retenir les principes suivants :

La part de l'aide réservée à ces pays doit rester modeste ;

- Les partenaires extérieurs doivent adopter la même attitude vis-à-vis du pays partenaire et coordonner leurs efforts de coopération pour le développement, ainsi que les actions et mesures qu'ils prennent dans d'autres domaines.

- L'aide doit servir à soutenir les institutions, nationales et locales, viables et les organisations de la société civile, en mettant l'accent sur l'élimination des obstacles à leur bon fonctionnement : il faut par exemple s'appliquer à relancer le dialogue sur les politiques à suivre, à soutenir les coalitions locales favorables à des réformes et à renforcer les capacités locales dans les domaines de la recherche et du dialogue social.

- La coopération pour le développement peut aussi viser à aider les autorités locales et les ONG à faire reculer la pauvreté dans des groupes de population précis afin d'atténuer leur vulnérabilité et de garantir la satisfaction de leurs besoins essentiels.

La façon de procéder face à ce genre de situation sera à déterminer entre tous les organismes qui ont affaire avec le pays partenaire en cause. Il sera important d'agir rapidement et de façon décisive. On pourra à cet effet recourir au dialogue sur les politiques à suivre, essayer d'établir un consensus entre les partenaires extérieurs, apporter un soutien aux coalitions locales favorables à une réforme, et œuvrer au renforcement des capacités locales de recherche et de dialogue social. L'évaluation des performances du pays partenaire dans l'application de sa stratégie de lutte contre la pauvreté permettra aux organismes de développement de déterminer s'il pourra prétendre dans l'avenir à une aide, et de quel type. Les pays performants peuvent s'attendre à recevoir une aide-programme accrue. Ceux qui ne le sont pas peuvent s'attendre à une limitation de l'aide qui leur est fournie (celle-ci pourra par exemple être ciblée, acheminée par des intermédiaires spécifiques, concentrée sur le renforcement des capacités, etc.). Cela aura inévitablement une incidence sur le volume de l'aide qui leur est accordée, du fait de l'augmentation des coûts de transaction et des délais d'acheminement qui en résultent pour les organismes de développement. 


\section{Aider les partenaires à élaborer des stratégies nationales valables de lutte contre la pauvreté}

\section{Le dialogue sur les politiques à suivre, au cœur de l'interaction entre les partenaires}

Le processus de dialogue sur les politiques à suivre au niveau local fait partie intégrante de la mise en place de partenariats solides et efficaces au service de la lutte contre la pauvreté. C'est dans ce cadre que doivent s'exprimer et se concilier les points de vue et les préoccupations de tous les partenaires concernant les problèmes de réduction de la pauvreté et les choix à faire. D'une façon générale, les accords et les décisions auxquels aboutit le dialogue sur les réformes économiques, politiques et administratives à opérer dans le pays partenaire détermineront les modalités de l'engagement de l'organisme de développement. Pour pouvoir négocier efficacement avec ses homologues locaux, celui-ci devra être bien informé de la situation locale au regard de la pauvreté.

Le dialogue sur les politiques à suivre doit permettre d'instaurer la confiance et de construire des partenariats à long terme autour d'objectifs communs. Dans une optique de réduction de la pauvreté, cela implique un dialogue plus global sur les divers types de politiques et programmes à appliquer au niveau national, sectoriel et local pour s'attaquer aux différents aspects de la pauvreté et à ses causes. Les principales questions et préoccupations à examiner dans le cadre d'un dialogue centré sur la lutte contre la pauvreté sont la promotion d'une croissance économique favorable aux pauvres (mesures économiques et structurelles et stratégies permettant d'assurer des moyens de subsistance durables), les mesures propres à favoriser l'équité, l'insertion sociale et le développement humain (prise en compte systématique de la problématique homme-femme, amélioration de la qualité et de la couverture des services sociaux de base) et les changements à apporter à la gestion des affaires publiques et aux institutions pour créer un environnement propice à la réduction de la pauvreté (démarginalisation, renforcement des droits et modes de gestion des affaires publiques favorables aux pauvres)*.

A court terme, surtout dans les pays qui n'ont pas encore totalement achevé la mise au point d'une stratégie de réduction de la pauvreté et ne se sont pas encore dotés d'institutions efficaces pour l'appliquer, un dialogue intense entre les organismes de développement et le pays va probablement rester nécessaire, faute de quoi on ne pourra espérer orienter les priorités et les résultats dans des directions plus favorables aux pauvres et aux femmes.

Les stratégies de lutte contre la pauvreté des pays partenaires comporteront des engagements implicites de leur gouvernement sur la base desquels les autres partenaires pourront mesurer les résultats obtenus en termes de modification des politiques et de réformes structurelles. Les organismes de développement et les gouvernements partenaires devront s'efforcer de se mettre d'accord sur les objectifs et sur les critères qui permettront de mesurer les progrès accomplis vers la réalisation de ces objectifs.

\section{Soutien des organismes de développement à la conception des stratégies nationales de lutte contre la pauvreté}

L'élaboration des stratégies nationales de lutte contre la pauvreté et des plans d'action correspondants nécessite souvent une aide, dont la nature et le calendrier peuvent être déterminants. Cette aide pourra prendre les formes suivantes :

- Une aide pour le diagnostic de la nature et des causes de la pauvreté afin d'informer au mieux la conception des mesures que doivent prendre les pouvoirs publics,
Les vues et préoccupations des différents acteurs et parties prenantes concernant les problèmes et choix stratégiques relatifs à la lutte contre la pauvreté doivent être débattues, conciliées et rapprochées.

Les organismes d'aide et les gouvernements partenaires doivent se mettre d'accord dès le départ sur des étalons permettant d'évaluer les progrès accomplis vers les objectifs assignés à la stratégie de lutte contre la pauvreté

\footnotetext{
*. Voir au chapitre 1 "Quelles sont les mesures à prendre ?”.
} 
Il est primordial de laisser aux pays partenaires le temps et la latitude dont ils ont besoin pour élaborer, sur la base d'une large concertation, leur propre stratégie de lutte contre la pauvreté. notamment grâce à une meilleure compréhension des problèmes sexospécifiques et de leurs conséquences pour les groupes vulnérables.

- Un soutien pour l'élaboration des grands axes autour desquels doivent s'articuler les programmes sectoriels et intégrés de lutte contre la pauvreté pour favoriser une décentralisation et un rôle actif des collectivités locales.

- Des ressources pour le suivi et l'évaluation de l'accès des pauvres aux services dans le cadre des programmes sectoriels.

- Un renforcement des capacités de production et d'analyse d'informations et de statistiques, y compris un soutien pour les évaluations de la pauvreté et la mise au point de techniques et outils statistiques appropriés (données ventilées par sexe, âge, groupe social, etc., par exemple).

- Une facilitation et un élargissement de la participation de la population au processus de mise au point des stratégies de lutte contre la pauvreté, par exemple en aidant à mettre sur pied la consultation des organisations de la société civile et des femmes et en soutenant les travaux des commissions parlementaires et législatives ou des instituts indépendants d'études politiques.

- Un renforcement des capacités de gestion et de comptabilité des flux d'aide, d'évaluation de leur impact, et d'apprentissage institutionnel.

- Un renforcement des capacités des instances décentralisées et locales afin de favoriser au maximum l'appropriation de la stratégie nationale de lutte contre la pauvreté.

Tout doit être fait pour laisser aux autorités du pays partenaire suffisamment de temps et de latitude pour élaborer, sur la base d'une large concertation, leur propre stratégie de lutte contre la pauvreté.

Les organismes de développement doivent respecter, en matière d'assistance technique, le principe d'intervention minimum. Les activités doivent être programmées et exécutées par le pays en développement (dans toute la mesure du possible), et contribuer véritablement au renforcement des capacités nationales et des compétences locales. Cela vaut en particulier pour les études qui sous-tendent les stratégies nationales de lutte contre la pauvreté. Les organismes de développement doivent coopérer étroitement avec les gouvernements partenaires et définir clairement avec eux les tâches et responsabilités des conseillers expatriés, lesquels doivent rendre compte directement aux autorités locales. Il faut veiller à ce que les travaux se déroulent dans le respect des priorités locales et à ce que les informations produites grâce à l'aide au développement soient communiquées à toutes les parties prenantes. Pour assurer ce rôle de facilitateur, la coopération technique dispose de toute une gamme d'instruments, parmi lesquels des missions d'experts locaux et internationaux de plus ou moins longue durée, susceptibles d'être associés à une aide financière.

\section{Renforcement des fondements empiriques des stratégies de lutte contre la pauvreté}

Les stratégies nationales de lutte contre la pauvreté doivent se fonder sur les meilleurs éléments d'information possible. Des efforts concertés sont nécessaires pour renforcer les capacités locales de collecte et de gestion de données en vue de l'établissement de diagnostics et de la définition des politiques qui conviennent. A cette fin, les organismes de développement peuvent apporter un concours direct et indirect à l'enrichissement des compétences et des connaissances, et assurer la liaison entre les pays partenaires et les institutions régionales ou internationales. Les secteurs où ce type d'intervention peut se révéler le plus utile sont la sécurité alimentaire (systèmes d'alerte précoce), l'agriculture, la pêche, l'environnement (données par satellite) et la santé. 
L'exploitation critique et analytique des données laisse à désirer tant au niveau des pays partenaires qu'au niveau des organismes de développement. L'importance qu'attachent ces derniers au problème de l'égalité homme-femme appelle de meilleures données empiriques sexospécifiques sur la situation dans les différents pays, indispensables pour analyser dans chaque pays la pauvreté chez les femmes et concevoir des mesures volontaristes en leur faveur. Il en va de même pour les liens entre l'environnement et la pauvreté.

Dans le même temps, le principe du partenariat implique deux priorités :

- Partager ouvertement et régulièrement avec les gouvernements partenaires, la société civile (du Sud et du Nord) et les autres organismes de développement tous les travaux d'analyse, afin de mettre en place les bases d'évaluations de la pauvreté collectives ou partagées.

- Travailler en étroite coopération avec les institutions nationales à tous les niveaux - afin de tirer profit des rares compétences disponibles, de bénéficier de synergies et de promouvoir un apprentissage actif - à l'élaboration de cadres communs de diagnostic.

\section{Collaboration avec d'autres partenaires}

Ratisser large. Il existe dans tout pays en développement une multitude de partenaires réels et potentiels dont les divers talents, compétences et énergies pourraient être mis au service de la lutte contre la pauvreté. Les ministères centraux, les collectivités territoriales, les groupes d'entraide, les organisations de la société civile (du Nord comme du Sud), les syndicats, les mouvements d'opposition, les organisations féminines, le secteur privé, le monde de la recherche, les groupes religieux, les organismes de développement bilatéraux et multilatéraux et les pauvres eux-mêmes, tous ces groupes doivent participer à la conception, au financement et à l'application des stratégies de lutte contre la pauvreté. L'objectif doit être d'associer tous les partenaires potentiels dans une action coordonnée et concertée.

La réalité politique de la lutte contre la pauvreté. Les efforts des pays partenaires pour réformer leurs structures et leurs politiques dans un sens favorable à la population pauvre auront une résonance politique et ne manqueront pas de susciter des tensions entre les groupes qui s'accrochent à leurs privilèges et à leurs rentes de situation et ceux qui sont plus ouverts à une réforme et à des mesures en faveur des pauvres. En tant qu'instruments de la coopération pour le développement, les organismes de développement ne peuvent échapper à ces tensions. Ils doivent être bien conscients que traiter avec divers acteurs dans un contexte économique, social et politique où les objectifs de réduction de la pauvreté ne sont pas nécessairement partagés par tous, posera des problèmes auxquels il n'y a pas de solution simple. En soutenant les efforts des pouvoirs publics pour engager le dialogue avec la société sur les options et les choix de développement, les organismes d'aide apprendront à mieux connaître la dynamique sociale et politique locale et pourront constituer des alliances et des partenariats stratégiques avec des personnes et des institutions ouvertes aux réformes. Le mieux à cet égard est d'encourager l'avènement d'une démocratie pluraliste et participative dans laquelle les pauvres ont voix au chapitre. Il peut également se révéler utile d'apporter un soutien aux parlements ou autres instances législatives, aux médias indépendants, aux instituts d'études politiques et aux organisations de la société civile.

La réalisation de
bilans collectifs et
partagés de pauvreté
et l'élaboration de
cadres communs de
diagnostic
constituent une
priorité à laquelle
doivent œuvrer
ensemble les
organismes
extérieurs et les
autorités locales.

autorités locales. 
Les changements structurels et les réorientations de l'action des pouvoirs publics relèvent, par essence, d' un processus politique et sont souvent source de controverses, Les organismes d' aide doivent s'appliquer à encourager le dialogue social et la diffusion de l'information et à soutenir les institutions et les personnes ouvertes à des réformes.

\section{Il faut redoubler d' efforts pour $s^{\prime}$ assurer que les consultations entre les pouvoirs publics et la société civile concernant les problèmes de pauvreté donnent lieu à de réels échanges permettant d' informer et d'orienter les choix qui seront en définitive retenus.}

Tirer le meilleur parti des apports des partenaires. Bien connaître les avantages comparatifs et les atouts des différents partenaires peut aider à mieux orienter leurs efforts respectifs. Ainsi, les organisations de la société civile peuvent contribuer à mobiliser les acteurs locaux, dynamiser les efforts d'entraide, acheminer des moyens supplémentaires, militer pour la responsabilisation et la participation et entrer en contact avec des groupes et communautés marginalisés. Le secteur privé a un rôle à jouer en tant que premier créateur d'emplois durables, fournisseur de ressources à l'appui du développement et promoteur des transferts de technologie et de savoir-faire. Les organisations syndicales peuvent œuvrer à l'octroi d'un emploi décent à chacun et à l'amélioration des conditions de travail. Les organismes multilatéraux peuvent offrir leurs capacités de recherche, des apports de ressources et un champ d'échanges mondial et régional. Les organismes bilatéraux peuvent apporter leur forte présence sur le terrain, leurs relations de longue date avec le gouvernement et les acteurs locaux, des ressources complémentaires et leur expérience politique du développement et de la gestion des affaires publiques.

Le rôle clé des associations. Les organisations non gouvernementales, de même que des organismes du secteur privé, tels que chambres de commerce et entreprises, peuvent lancer des initiatives nouvelles et efficaces pour réduire la pauvreté ; elles doivent donc bénéficier du soutien institutionnel et financier nécessaire pour mettre en œuvre leurs projets. Nombre d'ONG, par exemple, ont ouvert la voie dans le domaine des modes d'apprentissage informels, notamment pour les savoirs fondamentaux, mais aussi de la construction de la paix, des stratégies en faveur des femmes, des partenariats avec les collectivités locales et du renforcement des capacités des organisations de la société civile locale. Soutenir les activités militantes des ONG locales, des groupes de pression et des réseaux locaux qui représentent les pauvres est indispensable si l'on veut créer et maintenir un environnement propice à la réduction de la pauvreté en donnant la « parole » aux pauvres. L'amélioration de l'efficacité de ces acteurs sociaux passe d'abord par le renforcement de leurs capacités d'administration et d'analyse et par l'extension de leur champ d'action aux fractions les plus pauvres de la société.

Le partenariat implique un dialogue avec une multitude d'acteurs autres que le gouvernement. De nombreux partenaires se plaignent du manque d'ouverture du débat sur la pauvreté dans leurs pays. Il importe d'élargir à des partenaires plus nombreux et plus diversifiés le dialogue qui accompagne le travail de mise au point et d'application des stratégies de lutte contre la pauvreté. Il faudra s'attacher davantage à la qualité des consultations, en assurant une véritable participation préalable qui permette d'agir en sachant où l'on $v a$. Les organismes de développement peuvent apporter leur contribution à ce processus essentiel au plan de la logistique, de la planification, du recueil des informations, de la facilitation, de la coordination et de la diffusion qui seront nécessaires aux différents dialogues.

Dans leurs initiatives destinées à soutenir les efforts des gouvernements pour encourager une participation locale plus large au dialogue, les organismes de développement doivent veiller à ne pas porter atteinte à la légitimité des gouvernements partenaires et à rechercher dans la société civile des acteurs sérieux qui peuvent légitimement parler au nom des pauvres. On ne soulignera jamais assez la nécessité de respecter les efforts déployés par le pays partenaire pour construire et consolider ses propres institutions démocratiques. L'établissement de relations plus ouvertes avec la société civile est particulièrement important dans les pays où l'on considère encore que la politique du gouvernement ne va pas dans le bon sens ou que la stratégie locale de lutte contre la pauvreté n'est pas assez bien définie. Dans tous les cas, c'est un facteur important pour accroître la transparence de l'action du gouvernement aux yeux de l'ensemble de la société. 
Encadré 8. Comment les organismes bilatéraux peuvent optimiser leur collaboration avec les institutions multilatérales

Pour certains organismes bilatéraux, collaborer avec les institutions multilatérales et régionales est un moyen important d'accroître la portée et l'impact de leurs programmes d'aide. Il importe que tous les partenaires extérieurs s'efforcent d'établir entre eux des relations plus étroites et plus constructives, surtout en ce qui concerne les cadres stratégiques et les processus pilotés par le pays partenaire. Ils peuvent pour cela :

- Déterminer d'un commun accord avec les institutions multilatérales les rôles, responsabilités et obligations respectives des différents partenaires extérieurs dans les processus d'établissement des stratégies de lutte contre la pauvreté propres à chaque pays.

- Assurer des retours d'information du terrain au siège de l'organisme, afin de tenir la direction informée des perspectives, des enjeux et des difficultés de mise en œuvre.

- Participer activement aux dispositifs bilatéraux et multilatéraux de coordination de l'aide (Partenariat stratégique pour l'Afrique, groupes consultatifs, tables rondes, par exemple).

- Dans toute la mesure du possible, prendre des engagements à long terme fiables et transparents concernant les ressources allouées aux activités de lutte contre la pauvreté menées conjointement avec les institutions multilatérales.

- Se tenir au courant des missions et des possibilités de dialogue sur les politiques à suivre et prendre rapidement contact avec le personnel compétent des organismes multilatéraux.

- Veiller à ce que les réunions périodiques de coordination restent informelles, axées sur l'action concrète et les résultats (mise en commun de données d'expérience, examen des options et des scénarios possibles, demande de conseils, coordination des activités et des travaux de recherche, etc.).

- Rechercher des moyens de rationaliser et de simplifier les modalités de financement et de décaissement (chaque organisme de développement, par exemple, se portant volontaire pour financer un certain pourcentage d'un programme, à charge pour le gouvernement de fournir régulièrement des états financiers).

- Définir et gérer la coopération entre institutions bilatérales et multilatérales en matière d'assistance technique.
Assurer une véritable participation des acteurs locaux n'est pas une tâche facile. Travailler avec les partenaires et acteurs locaux demande beaucoup de temps et de personnel et ne va pas sans risque (en ce sens que cela n'aboutit pas nécessairement aux résultats attendus). Il est parfois très difficile de faciliter et de gérer la participation, compte tenu des rigidités de la programmation et des contraintes budgétaires qui continuent de peser sur les programmes et projets de coopération pour le développement. Cela est pourtant essentiel pour garantir l'appropriation locale, la durabilité et l'efficacité de l'action engagée. Les organismes de développement doivent donc s'attaquer de front au problème de la participation et de l'autonomisation des femmes, des groupes marginalisés et exclus et des plus pauvres parmi les pauvres. Assurer cette participation nécessite des systèmes efficaces de suivi et d'information en retour. Plus généralement, les organismes de développement doivent soutenir les efforts des gouvernements partenaires pour institutionnaliser la participation de l'ensemble de la société, et mettre sur pied des processus et mécanismes participatifs permettant de dépasser le niveau purement local.

Resserrer les liens avec les partenaires multilatéraux. Les organismes de développement doivent établir une collaboration plus étroite et plus collégiale avec les partenaires multilatéraux, aussi bien au niveau de leurs organes de décision que sur le terrain (encadré 8). Pour que la collaboration soit efficace, les organismes bilatéraux doivent placer dans une optique davantage tournée vers l'action leurs analyses des politiques des institutions multilatérales et être prêts à les remettre en cause lorsqu'il apparaît qu'elles portent préjudice aux pauvres.
Travailler avec les partenaires et les acteurs locaux demande du temps et des ressources humaines et ne va pas sans risque, autant de problèmes supplémentaires pour les organismes d'aide du fait des contraintes qui leur sont imposées en matière de programmation, d'effectifs et de budget.

Les organismes bilatéraux devraient placer dans une optique davantage tournée vers l'action leur analyse des politiques des institutions multilatérales et à remettre en cause celles qui portent préjudice aux pauvres. 
Le défi que doivent relever les organismes d' aide consiste à trouver des moyens de collaborer sans saper I'appropriation locale ni imposer des charges supplémentaires aux pays partenaires.

\section{Améliorer la coordination de l'aide à l'appui des stratégies nationales de lutte contre la pauvreté}

Problèmes de coordination. Dans un partenariat, la coordination de l'aide incombe à l'ensemble des parties, mais c'est le gouvernement partenaire qui doit en avoir l'initiative". Les organismes bilatéraux doivent constamment veiller à partager entre eux les informations et le travail, afin d'assurer la cohérence de leurs messages et de concentrer leurs efforts sur les besoins essentiels et sur les possibilités de collaboration. En même temps, il est capital que les organismes multilatéraux communiquent leurs informations aux autres partenaires afin de favoriser une véritable coordination et de leur permettre de faire un meilleur usage de leurs cadres d'action. Une plus large délégation de pouvoir aux bureaux locaux permettra à ceux-ci d'avoir l'initiative et la marge de manœuvre nécessaires pour renforcer et améliorer la coopération et la coordination sur le terrain. La coordination doit englober les organismes bilatéraux et multilatéraux et s'opérer aux niveaux national, régional et international.

La difficulté pour la communauté du développement est de trouver des moyens de collaborer sans empêcher l'appropriation locale ni imposer des charges supplémentaires aux pays partenaires.

En même temps, il faut que les organismes de développement portent davantage d'attention à la cohérence, aussi bien à travers la communauté du développement qu'au sein de leurs propres structures (qu'il s'agisse d'un ou de plusieurs ministères ou organismes). Une plus grande cohérence entre les organismes de développement et les ministères représentés dans les organes directeurs des institutions multilatérales est indispensable si l'on veut réduire les risques de contradictions et améliorer la cohérence interne de la politique générale des Membres du CAD à l'appui du développement et de leurs efforts de réduction de la pauvreté. Chaque organisme doit rester attentif aux risques de contradictions, de double emploi, de recoupement de ses activités avec celles d'autres partenaires extérieurs - et au sein de ses propres structures - aussi bien dans le dialogue sur les politiques à suivre que dans la programmation. Une plus grande harmonisation des pratiques est nécessaire aux niveaux bilatéral, régional et international, mais elle exige de la part de tous les organismes la volonté de travailler ensemble pour maximiser leurs avantages comparatifs respectifs, déterminer la meilleure répartition du peu de moyens disponibles et assurer la cohérence globale de l'aide au développement acheminée vers le pays partenaire en cause.

Les groupes consultatifs et les tables rondes sont des mécanismes de coordination de l'aide qui peuvent en même temps renforcer les capacités du pays partenaire et son appropriation des programmes. Ces forums doivent être pilotés par le pays partenaire, et faire une place importante à la lutte contre la pauvreté. Il faut faciliter la tenue, dans les pays partenaires, de ces réunions auxquelles les organismes de développement doivent envoyer des représentants de haut niveau.

\section{Priorités d'action des organismes bilatéraux}

Ce chapitre 2 vient d'exposer les principes de base du partenariat et la façon dont les organismes de développement peuvent les appliquer dans divers contextes nationaux afin d'aider leurs partenaires à élaborer et mettre en œuvre de solides stratégies nationales de lutte contre la pauvreté.

*. Dans ce contexte idéal, la stratégie de lutte contre la pauvreté, le budget national et le cadre budgétaire à moyen terme sont des instruments importants pour structurer et coordonner l'aide au développement (chapitre 3). 
Après avoir examiné ces concepts, les Membres du CAD ont recensé un certain nombre de priorités pour l'action des organismes de développement, en particulier sur le terrain. Ceux-ci doivent en particulier veiller plus résolument à :

Soutenir des stratégies de lutte contre la pauvreté qui soient prises en charge et pilotées par les pays concernés, et se fonder sur les besoins et priorités recensés dans ces stratégies pour la programmation de leurs activités.

- Affecter une part plus importante de l'aide au développement aux pays où s'offrent les meilleures chances de faire reculer la pauvreté compte tenu du nombre de personnes vivant dans la pauvreté absolue, de la détermination des pouvoirs publics à combattre la pauvreté et des résultats constatés de l'action menée.

- Réduire la charge que l'aide au développement impose aux partenaires locaux en regroupant les efforts (missions conjointes, recherche en collaboration, diagnostics communs, partage des coûts, etc.), en allégeant les exigences administratives (par exemple en simplifiant, en rationalisant et, dans toute la mesure du possible, en harmonisant les formalités et procédures ; en acceptant, chaque fois que c'est possible, les modèles de documents et de stratégies établis par le partenaire) et en coordonnant leurs approches et leurs actions.

- Consacrer le temps et les moyens nécessaires à l'édification, sur une base de réciprocité, de véritables partenariats au service de la lutte contre la pauvreté, et les doter de mécanismes et de réseaux efficients au meilleur coût.

- Adapter leurs structures et leurs méthodes de travail aux enjeux et aux besoins des partenariats dans le domaine de la lutte contre la pauvreté (par exemple accroître et étoffer la présence sur le terrain ; assouplir la prise de décision ; accepter les modèles de documents et de stratégies établis par le partenaire ; renforcer la transparence et les obligations de comptes aux autres partenaires; allonger les calendriers de programmation, développer les aptitudes du personnel en matière d' « accompagnement » et de recherche de consensus).

- Euvrer plus activement au développement des capacités humaines et institutionnelles des pays partenaires (par exemple pour améliorer la gestion des affaires publiques et promouvoir la responsabilisation, permettre aux partenaires de gérer l'aide au développement et d'en rendre compte, encourager l'utilisation des technologies de l'information et des communications, etc.).

- Intégrer la problématique homme-femme dans l'ensemble des politiques, programmes, instruments et modalités d'aide (au lieu d'en faire un chapitre séparé).

- Intégrer l'objectif du développement durable, notamment les problèmes d'environnement, dans les cadres stratégiques de lutte contre la pauvreté.

Adopter, dans toute la mesure du possible, un échéancier pluriannuel pour la programmation et le financement de la lutte contre la pauvreté, afin d'accompagner les budgets et programmes pluriannuels du gouvernement partenaire.
Pour édifier des

partenariats au

service de la lutte

contre la pauvreté, les organismes

d'aide doivent en

priorité :

... Soutenir des stratégies de lutte contre la pauvreté que les pays partenaires fassent leurs et dont ils assurent la conduite,...

... affecter les ressources là où elles auront le plus d'impact sur la pauvreté,...

... rationaliser et simplifier la gestion de l'aide et collaborer pour I'accomplissement de missions similaires,...

... renforcer les capacités institutionnelles locales de mise en œuvre des politiques et de gestion des apports d' aide,...

... assouplir et allonger I'horizon temporel des transferts de ressources... 
... et évaluer leurs propres

performances au

regard de la

contribution que leur action a apportée au recul de la pauvreté.
- Évaluer les résultats de la coopération pour le développement du point de vue de l'ampleur et de l'intensité de la réduction de la pauvreté, et élaborer les systèmes et méthodes de suivi et d'évaluation requis.

- Favoriser et appuyer les efforts locaux (notamment de la société civile, des parlements et autres instances législatives, des organismes publics) de suivi des programmes de lutte contre la pauvreté et de l'utilisation faite des allégements de dette.

- Encourager les pays à mettre au point des indicateurs et des objectifs locaux de réduction de la pauvreté et renforcer les capacités locales en matière de statistique, d'analyse et d'évaluation. 


\section{Cadres et instruments de programmation par pays}

\section{Introduction}

Les procédures et les choix en matière de programmation par pays ont un rôle essentiel à jouer dans la lutte contre la pauvreté. Ce troisième chapitre décrit les cadres qui se mettent en place dans les pays partenaires afin d'étayer la formulation, la mise en œuvre et le suivi de stratégies nationales centrées sur des objectifs de réduction de la pauvreté. Il expose ensuite le rôle nouveau que peuvent jouer les stratégies par pays établies par les organismes bilatéraux pour renforcer le ciblage et l'impact, sur la pauvreté, des activités menées dans chaque pays. Enfin, il passe en revue les avantages relatifs des principaux instruments de coopération pour le développement et les bonnes pratiques qui commencent à se faire jour pour renforcer l'efficacité de l'aide au service de la lutte contre la pauvreté.

\section{Cadres stratégiques qui se mettent en place dans les pays partenaires}

Pour l'établissement des programmes par pays, il faut avant tout s'appuyer sur les cadres stratégiques et instruments de planification déjà en place dans les pays partenaires. Ces dispositifs nationaux doivent être utilisés pour formuler les politiques, fixer les priorités, arrêter les décisions qu'elles requièrent en matière de dépenses et étudier de façon suivie les résultats de la lutte contre la pauvreté. La présente section revient sur plusieurs des cadres de développement que la communauté internationale prône depuis plusieurs années. Elle met aussi en lumière les défis particuliers que doivent relever les organismes de développement qui fondent leurs efforts de coopération sur ces instruments.

\section{Cadres stratégiques de portée nationale à l'appui du développement}

Un large consensus se dégage au sein de la communauté internationale quant à la nécessité d'élaborer, sous la conduite des pays, des cadres globaux de développement intégrant une stratégie nationale de lutte contre la pauvreté. Ce constat a amené diverses instances internationales à proposer plusieurs cadres ayant entre eux des liens étroits et s'imbriquant souvent les uns dans les autres. On peut ainsi notamment citer les Stratégies nationales de développement durable (SNDD) issues de la Conférence des Nations unies sur l'environnement et le développement qui s'est tenue en 1992, les Bilans communs de pays prévus dans le Plan-cadre des Nations unies pour l'aide au développement (UNDAF) dont l'expérimentation a débuté en 1997, le Cadre de développement intégré (CDI) lancé par la Banque mondiale au début de 1999, et les Cadres stratégiques de lutte contre la pauvreté (CSLP) requis par les institutions de BrettonWoods depuis fin 1999.

Ensemble, ces cadres, sous les diverses formes qu'ils revêtent au niveau national, sont des outils stratégiques conçus pour permettre la traduction des objectifs nationaux et internationaux de développement en actes. Il convient de s'appliquer à faire en sorte qu'ils couvrent tous les domaines où une action est nécessaire, favorisent un recul de la pauvreté et soient compatibles avec les autres stratégies nationales. Dans l'idéal, les pays partenaires devraient avoir un plan national unique qui intégrerait les priorités
Les cadres de développement élaborés sous la conduite des pays partenaires sont des instruments déterminants pour intégrer les priorités économiques, sociales et environnementales... 
... dans une stratégie d'ensemble axée sur la réduction de la pauvreté.

\section{La stratégie de lutte contre la pauvreté doit résulter de I'initiative du pays intéressé, se fonder sur la participation, prendre en compte la globalité des problèmes à régler et être axée sur I'obtention de résultats.}

économiques, sociales et environnementales dans un cadre stratégique global visant à inscrire dans la durée le processus de développement dans son ensemble - y compris le recul de la pauvreté ${ }^{1}$.

Compte tenu de la charge supplémentaire qu'impose aux pays partenaires l'obligation de respecter de multiples cadres de programmation, la communauté internationale devrait rationaliser les divers documents et plans économiques actuellement établis par les pays partenaires, les organisations internationales et les organismes bilatéraux. Ces derniers doivent apporter un soutien à l'élaboration des stratégies nationales en concourant au renforcement des capacités locales en matière de formulation des politiques, de conduite des processus consultatifs, ainsi que de recherche et d'analyse (chapitre 2). Les stratégies définies au moyen de ces cadres, et en particulier la stratégie de lutte contre la pauvreté fondée sur l'initiative du pays, doivent servir de référence aux organismes d'aide pour la programmation des activités de lutte contre la pauvreté qu'ils entendent mener à l'échelon de chaque pays.

\section{Stratégie de lutte contre la pauvreté fondée sur l'initiative du pays}

L'adoption généralisée d'une approche consistant dans l'élaboration d'une stratégie de lutte contre la pauvreté (SLP) fondée sur l'initiative du pays concerné soulève un certain nombre de problèmes liés aux considérations suivantes : le degré d'appropriation et d'engagement manifesté par le pays partenaire, sa capacité de concevoir des plans d'action assortis de priorités hiérarchisées et l'aptitude de la société civile à collaborer avec les pouvoirs publics (ainsi que la volonté de ces derniers d'associer la société civile à leur action), l'existence de données sur la pauvreté et leur qualité, et les difficultés que pose la détermination d'une croissance favorable aux pauvres. Mais il y a aussi là une occasion particulièrement propice à la promotion d'une meilleure intégration des questions de viabilité écologique dans les stratégies de lutte contre la pauvreté qui se font jour. Au bout du compte, il faudrait que les SLP fondées sur l'initiative des pays présentent toutes les caractéristiques de stratégies à long terme écologiquement viables.

Dans les cas où les pays à faible revenu sont tenus de se doter d'un Cadre stratégique de lutte contre la pauvreté (CSLP) pour les besoins de l'Initiative PPTE (pays pauvres très endettés) renforcée et parce qu'ils souhaitent obtenir de la Banque mondiale et du FMI une aide assortie de conditions de faveur, le CSLP devrait normalement faciliter et améliorer les efforts nationaux de lutte contre la pauvreté, et non faire double emploi avec eux et les fragiliser.

La stratégie de lutte contre la pauvreté doit résulter de l'initiative du pays intéressé, reposer sur un processus participatif, prendre en compte la globalité des problèmes à régler et être axée sur l'obtention de résultats. Dans leur action à l'appui du processus SLP, qui est relativement nouveau et n'a encore guère été éprouvé, les organismes de développement ne doivent pas perdre de vue les considérations suivantes: il convient d'accepter que les SLP prennent des formes et répondent à des normes différentes; le rythme du processus SLP doit être adapté aux capacités du pays intéressé, de manière à susciter un renforcement de l'appropriation locale; il ne doit pas être influencé par la pression des facteurs qui poussent au décaissement des fonds. Il convient de faire preuve d'une certaine souplesse et d'adopter une optique à long terme concernant les attentes à placer dans le processus SLP, étant donné qu'une réduction durable de la pauvreté ne s'obtient pas du jour au lendemain. Ce processus doit être ouvert aux organismes bilatéraux qui doivent y être pleinement intégrés compte tenu des avantages comparatifs dont ils peuvent se prévaloir au niveau de la planification, de la mise en œuvre, du suivi et de l'évaluation des stratégies de lutte contre la pauvreté. 


\section{Budgets nationaux et examens des dépenses publiques}

Le budget national (ou infranational) constitue l'instrument le plus important pour hiérarchiser les objectifs assignés à la stratégie nationale de lutte contre la pauvreté et rendre compte des résultats qu'elle produit. Il donne une prévision des dépenses et des recettes de l'année, qui offre l'avantage de refléter les priorités de l'action gouvernementale, chiffre le coût des mesures envisagées pour obtenir un résultat particulier, et fixe l'enveloppe globale des ressources qui semblent pouvoir être dégagées sans compromettre les grands équilibres macro-économiques. Lorsque c'est faisable, les engagements financiers consentis par les organismes d'aide doivent être intégrés au budget, afin de rendre plus transparente l'affectation des ressources extérieures et de permettre aux pouvoirs publics de décider des priorités au service desquelles sera mise une fraction plus importante de l'aide au développement. De ce point de vue, les examens des dépenses publiques réalisés en partenariat avec les organismes extérieurs ont un rôle essentiel à jouer pour assurer une utilisation des ressources qui soit favorable aux pauvres, conserver au secteur public une taille rationnelle et cibler les groupes vulnérables.

\section{Cadres budgétaires à moyen terme}

Dans les pays partenaires qui possèdent les capacités requises pour établir un cadre budgétaire à moyen terme, les organismes d'aide peuvent aussi s'appuyer sur cet instrument national de prospective. Un cadre budgétaire à moyen terme peut se révéler utile pour la lutte contre la pauvreté, car il permet de raccorder plus étroitement les objectifs assignés à la stratégie nationale de lutte contre la pauvreté aux recettes et aux dépenses, et sert en même temps de référence pour l'établissement des budgets annuels. C'est un outil qui peut grandement améliorer la transparence dès lors que sont établis des indicateurs et mis en place des mécanismes explicites à l'aide desquels déterminer si les dépenses ont bénéficié à ceux au profit desquels elles sont censées avoir été consenties.

Les examens budgétaires périodiques peuvent devenir une bonne occasion pour les pouvoirs publics et leurs partenaires de faire le point ensemble sur leur action commune au service de la lutte contre la pauvreté. Le cadre budgétaire à moyen terme n'est toutefois que l'un des divers instruments importants susceptibles d'être mis au service de la réduction de la pauvreté. Il n'est pas axé sur les résultats ou sur des questions qui sortent du domaine budgétaire telles que les politiques de développement ou le rôle du secteur privé.

Les premiers enseignements qui se dégagent des stratégies de lutte contre la pauvreté fondées sur l'initiative des pays font ressortir la nécessité pour les organismes de développement de prendre des engagements à plus long terme. Dès lors que la politique suivie leur convient, ces organismes devraient accepter que leurs apports soient pris en compte dans les examens budgétaires, ce qui témoignerait de leur volonté d'inscrire cette aide dans la durée.

\section{Stratégies par pays élaborées par les organismes d'aide}

La tendance des pays partenaires à se doter de cadres stratégiques de lutte contre la pauvreté devrait contribuer à affiner le ciblage des activités de programmation des organismes d'aide. L'objectif doit être, à long terme, de réduire la place des stratégies par pays élaborées par ces organismes eux-mêmes. Dans le meilleur des cas, ces stratégies-pays deviendront un simple «plan d'activité » pour la mise en œuvre des stratégies nationales de lutte contre la pauvreté fondées sur l'initiative des pays partenaires. En attendant, les organismes d'aide continueront de s'appuyer sur les mécanismes de planification qui leur sont propres pour organiser et adapter leur aide en fonction de la situation qui prévaut dans chaque pays partenaire.

La stratégie de lutte contre la pauvreté doit servir de référence à tous les organismes d'aide pour la programmation et la mise en œuvre de 


\section{Encadré 9. Plan possible d'une stratégie-pays bilatérale axée sur la réduction de la pauvreté}

1. Caractéristiques de la pauvreté dans le pays considéré (nature, causes, évolution, qui sont les pauvres et où vivent-ils).

2. Évaluation de la volonté du pays considéré de lutter contre la pauvreté.

3. Récapitulatif des principales composantes de la stratégie nationale de lutte contre la pauvreté écologiquement viable (par exemple secteurs, cadre politico-administratif, participation/ consultation, ainsi qu'objectifs précis, indicateurs, suivi et évaluation).

4. Stratégie que se propose de suivre l'organisme d'aide

Questions à aborder dans le dialogue sur les mesures à prendre (priorités, partage des responsabilités, performance, gestion des affaires publiques, etc.).

Enseignements pertinents et bonnes pratiques qui se dégagent de l'expérience de l'organisme d'aide ou de celle d'autres instances.

Grands axes du soutien accordé (et éléments les justifiant au regard de la stratégie nationale de lutte contre la pauvreté, de l'avantage comparatif de l'organisme d'aide, des efforts connexes de renforcement des capacités) :

- Croissance économique favorable aux pauvres : rythme et qualité.

- Démarginalisation, droits et gestion des affaires publiques favorable aux pauvres.

- Services sociaux de base à l'appui du développement humain.

- Sécurité humaine : réduire la vulnérabilité et gérer les crises.
- Intégration de la problématique homme-femme et promotion de l'égalité entre les sexes.

- Intégration de la nécessité d'assurer la pérennité de l'environnement par l'instauration de modes d'utilisation durable des moyens de subsistance.

Modes d'intervention et financement des activités :

Équilibre entre les activités directement/indirectement ciblées sur les pauvres (y compris au moyen de mécanismes de ciblage), les actions indirectes ou globales en direction de populations entières comprenant des pauvres (approches sectorielles, par exemple) et les initiatives de caractère structurel plus vastes destinées à promouvoir une croissance favorable aux pauvres.

- Équilibre entre le soutien budgétaire (dans le cadre du budget général, d'un fonds pour la lutte contre la pauvreté, d'un soutien sectoriel) et les dons et prêts accordés au titre de projets, compte tenu des perspectives d'évolution du niveau d'endettement.

Mécanismes de coordination (avec les pouvoirs publics, les autres organismes d'aide extérieurs, les acteurs locaux).

5. Problèmes de cohérence des politiques

6. Indicateurs de performance (provenant autant que possible d'un ensemble commun d'indicateurs défini de façon concertée par les organismes d'aide et les autorités du pays partenaire).

7. Critères d'auto-évaluation de la stratégie-pays (jalons précis, mesurables et assortis d'une date butoir permettant de suivre l'avancement de la mise en œuvre de la stratégie-pays)

Note : Les éléments 1 à 3 sont à dégager directement des documents relatifs à la stratégie de lutte contre la pauvreté du pays partenaire.

Les conditions essentielles auxquelles doit répondre l'élaboration d'une stratégiepays ciblée sur la pauvreté découlent des principes de base énoncés dans les chapitres 1 et 2. Plus précisément, les stratégies-pays doivent :

S'articuler autour d'objectifs de réduction de la pauvreté, notamment la recherche d'une croissance économique favorable aux pauvres, et se justifier au regard de ces objectifs, la hiérarchie étant appelée à exercer un contrôle attentif.

- S'inscrire dans le droit fil des stratégies de lutte contre la pauvreté arrêtées par les pays partenaires, lesquelles doivent servir de référence pour la programmation et la mise en œuvre des activités de tous les intervenants.

- Tenir compte de l'action engagée par les autres organismes d'aide dans le pays considéré et des mécanismes de coordination de l'aide qui peuvent y exister.

- Présenter un caractère réellement stratégique et reposer sur les meilleures informations possible concernant la situation du pays intéressé au regard de la pauvreté, grâce notamment à la réalisation de bilans de pauvreté, d'analyses des sexospécificités et autres facteurs sociaux, et d'examens portant sur les liens entre état de l'environnement et pauvreté (encadré 9). 


\section{Domaines sur lesquels doit porter une coopération axée sur la pauvreté}

Des efforts de coopération pour le développement axés sur la pauvreté, quelle que soit leur forme - aide-programme, aide sectorielle, projets, coopération technique ou dialogue sur les mesures à prendre - doivent porter sur les grands domaines d'action énoncés dans le chapitre 1. Les exemples qui suivent ont pour but de montrer le type de réformes engagées par les pays partenaires qui méritent un soutien particulier dans l'optique de la lutte contre la pauvreté.

La gestion des affaires publiques est un domaine dans lequel les organismes bilatéraux peuvent jouer un rôle important en soutenant la démarginalisation par la participation, le renforcement de la transparence, la lutte contre la corruption et la recherche d'une efficience accrue dans la gestion du secteur public. Au niveau de la gestion des affaires publiques, le principal problème qui se pose dans l'optique de la réduction de la pauvreté est de faire en sorte que les pauvres des deux sexes influent davantage sur l'élaboration des politiques et accèdent plus facilement à des services de base d'une qualité convenable. En ce qui concerne ce dernier point, l'accroissement des possibilités d'accès des femmes à ces services nécessite une attention, des stratégies et des apports de ressources particuliers.

Une réforme de la fonction publique propre à améliorer l'efficience de la gestion du secteur public et des services fournis par ce secteur est essentielle pour la conception et la mise en œuvre de politiques favorables aux pauvres. Cette réforme doit contribuer à renforcer la transparence par l'instauration de modes de sélection, de nomination et de promotion des fonctionnaires selon leur mérite, de codes de conduite et de mécanismes propres à en assurer le respect, de dispositifs garantissant un examen minutieux des politiques gouvernementales et de leurs résultats par des organes élus, ainsi que de procédures de suivi et d'obligations de comptes concernant l'efficacité et la qualité des services rendus. Cette réforme doit aussi favoriser la démarginalisation grâce à la mise en place de services publics qui répondent aux besoins de la population, à l'adoption de méthodes nouvelles de consultation des personnes qui vivent dans la pauvreté, et à la définition de normes minimales garantissant un accès équitable pour un prix raisonnable aux services de base.

Une réforme de la gestion budgétaire est capitale pour rendre plus favorable aux pauvres la répartition des ressources entre les secteurs, les régions et les diverses catégories de la population, ainsi qu'à l'intérieur de chacun d'eux. Les réformes qui présentent un intérêt particulier pour la lutte contre la pauvreté sont notamment : (i) la réforme des administrations des impôts et des douanes, qui ont un rôle essentiel à jouer dans l'amélioration de la gestion des affaires publiques, la préservation de la stabilité économique et, surtout, l'augmentation des ressources susceptibles d'être orientées vers les pauvres ; (ii) les réformes institutionnelles, par exemple la modification de la législation fiscale, la mise en place d'une administration fiscale, la réalisation d'examens des dépenses publiques, l'amélioration de la gestion des finances publiques et le renforcement des services des comptes publics; (iii) la réforme de la politique fiscale en vue d'une meilleure affectation des ressources par la recherche d'une efficacité plus grande dans le recouvrement de l'impôt et l'amélioration des effets redistributifs de la fiscalité.

La décentralisation peut contribuer à améliorer la qualité des services utiles aux pauvres et à leur en faciliter l'accès. Elle permet d'associer plus étroitement les représentants des pauvres à l'action des pouvoirs publics, et ainsi d'orienter davantage cette dernière vers les besoins des pauvres, d'accroître sa transparence et de mieux assurer la pérennité
Les réformes de la gestion des affaires publiques méritent un soutien particulier dans l'optique de la lutte contre la pauvreté. 
de ses résultats. Elle favorise aussi la délégation légitime de pouvoir dans le cadre de systèmes politiques de type fédéral. Elle renforce le sentiment d'appartenance et facilite l'ajustement des projets sur les réalités locales. La décentralisation présente cependant aussi des risques, qui ne doivent pas être sous-estimés : les pouvoirs locaux peuvent être accaparés par les élites locales, les disparités régionales peuvent s'aggraver, ou l'administration centrale peut se décharger de ses responsabilités à l'égard des pauvres. Il importe donc de déterminer le degré de décentralisation et le calendrier de ce processus en tenant compte de la situation particulière du pays considéré sur le plan national et, parfois, infranational, notamment de la nécessité de maintenir un juste équilibre entre les différents niveaux, de faire intervenir les acteurs concernés et d'évaluer les capacités locales. La coopération technique peut se révéler un moyen utile d'aider à arbitrer les conflits d'intérêts et de renforcer les capacités des diverses catégories d'acteurs.

Une réforme du régime foncier est indispensable pour faire reculer la pauvreté dans bon nombre d'économies fondées sur l'agriculture; elle doit se traduire par une modification de la législation qui permette de mieux garantir les droits de propriété et d'éliminer les différences existant à cet égard entre hommes et femmes. La réforme agraire est une question très délicate. Lorsqu'elle est réalisable du point de vue politique, elle peut contribuer dans une large mesure à assurer une répartition plus équitable des biens fonciers. Dans ce domaine, les bonnes pratiques consistent, entre autres, à faire usage d'instruments conformes aux lois du marché, à adopter une législation qui garantisse la sécurité d'occupation des terres, à encourager le partenariat entre secteurs public et privé en vue de soutenir la production agricole par la formation, l'apport de ressources financières et la technologie, et à relier la réforme foncière à la décentralisation.

Le soutien de la société civile est déterminant pour faire reculer la pauvreté. Les organismes bilatéraux possèdent un avantage comparatif pour la promotion du pluralisme dans la société civile par le soutien d'organisations professionnelles (comme les associations de petits exploitants agricoles, les syndicats) et de groupes de défense des droits de l'homme. Ces organismes locaux peuvent être de puissants agents de changement car ils sont bien placés pour comprendre les besoins et prendre en main les intérêts des populations politiquement marginalisées (minorités ethniques, femmes, paysans sans terre, habitants des bidonvilles). Il est essentiel de consolider le soutien apporté à la cause des droits de l'homme, afin de donner aux pauvres les moyens d'améliorer leurs conditions de vie, et à la société civile la possibilité de critiquer les politiques injustes ou inefficaces et de les faire réformer. Cet effort est particulièrement important pour le combat des femmes contre la discrimination dont elles sont l'objet. L'existence d'organes d'information indépendants facilitera considérablement ce processus. Le dialogue sur les politiques à suivre, surtout aux niveaux les plus élevés des sphères politique et administrative, constitue un cadre approprié pour attirer l'attention sur le problème des droits humains, ainsi que sur certaines actions et réformes des pouvoirs publics.

Le développement du secteur privé a également une très grande importance, étant donné les effets positifs qu'un secteur privé dynamique est susceptible d'exercer sur la pauvreté, car source de création d'emplois, de richesses et de savoir-faire et vecteur d'intégration des femmes et des groupes défavorisés dans la vie économique. Les organismes de développement peuvent soutenir l'application de mesures visant à assurer la mise en place d'un cadre juridique propice à réduire les obstacles administratifs et réglementaires à l'investissement, à renforcer le secteur financier et à encourager la privatisation des entreprises d'État dans des conditions concurrentielles. Des approches adaptées à chaque cas peuvent permettre d'orienter davantage le contenu et les résultats de ces mesures dans un sens favorable aux pauvres. Par exemple, le produit des privatisations peut servir à financer la création de fonds de promotion de la micro-entreprise au profit des 
travailleurs dont l'emploi a été supprimé, à soutenir les établissements de micro-crédit ou à développer les infrastructures utiles au secteur informel et aux populations pauvres.

La réalisation d'analyses politiques par pays afin de déterminer les retombées politiques de telle ou telle mesure ou programme est essentielle pour la sélection des domaines auxquels l'aide doit être consacrée.

\section{Principaux instruments de coopération financière et technique}

Les organismes de développement se servent de différents instruments de coopération pour aider les pays partenaires à lutter contre la pauvreté, entre autres des programmes et projets d'envergure nationale, régionale ou sectorielle, généralement associés à un dialogue à tous les niveaux sur les politiques à suivre et étayés par des activités de coopération technique. Un allégement de la dette, des apports d'aide humanitaire ou un soutien de la coopération régionale peuvent aussi être proposés dans les situations difficiles ou particulières.

Les pays partenaires se trouvant dans des situations très diverses, on ne peut affirmer qu'une forme de coopération pour le développement aura dans tous les cas un effet de réduction de la pauvreté plus grand qu'une autre. Cela dit, lorsque le contexte politique, économique et institutionnel est propice, l'aide-programme peut constituer un mode de prestation efficace pour amener un recul massif de la pauvreté si elle est associée à des apports d'un volume appréciable. Les projets, qui touchent un nombre limité de personnes, peuvent influer durablement sur les moyens d'existence de ceux qui en bénéficient, à condition qu'ils soient financièrement viables et compatibles avec le cadre macroéconomique. Le recentrage qu'ont opéré les organismes de développement sur la lutte contre la pauvreté a relancé le débat stratégique sur les avantages relatifs de l'aideprogramme et de l'aide-projet. Il en résulte qu'une place grandissante est faite au soutien aux programmes sectoriels, et que l'on s'attache maintenant à accroître en mettant l'accent sur le rôle fondamental de l'appropriation par les partenaires au maximum l'efficacité des projets locaux.

Étant donné l'ampleur des effets de synergie que peut produire la conjonction de différentes formes de coopération pour le développement aux niveaux macro-économique, méso-économique et micro-économique, les programmes par pays devraient dans l'idéal s'appuyer sur l'association de divers instruments. La nature de ces derniers et la part respective de l'action directe et indirecte, ciblée et de portée générale, doivent découler d'une analyse des besoins de chaque pays, réalisée dans le cadre d'un dialogue avec le gouvernement et les autres parties prenantes, comme il est indiqué dans le chapitre 2.

\section{Aide-programme}

L'aide-programme consiste à apporter à un pays partenaire des contributions financières, par exemple un soutien à la balance des paiements ou un soutien budgétaire, visant à faciliter le développement d'une manière générale sans qu'aucun lien soit établi avec des projets particuliers. Elle est souvent associée à la promotion de réformes au niveau macro-économique et/ou dans des secteurs particuliers.

Le rôle qui revient à l'aide-programme dans la lutte contre la pauvreté consiste à fournir, de la façon la plus efficiente possible, des ressources financières à un pays qui a la volonté et est concrètement à même d'en faire un usage efficace pour la mise en œuvre d'une stratégie rationnelle visant à faire reculer la pauvreté. C'est une forme de coopération pour le développement qui signifie que l'organisme d'aide concerné est prêt, en reconnaissance de l'amélioration des politiques, à laisser au pays partenaire la
Au vu des grandes possibilités de synergie qui existent, les organismes d'aide doivent faire appel à un ensemble d'instruments en associant à l'aideprogramme et au soutien sectoriel un dialogue sur les politiques à suivre et des activités de coopération technique.

L'aide-programme peut ouvrir la voie à un dialogue permanent sur les mesures favorables aux pauvres à prendre dans les domaines capitaux que sont la réforme macroéconomique et la bonne gestion des affaires publiques. 
Les nouvelles approches du soutien sectoriel devraient contribuer à favoriser l'appropriation locale et la participation à tous les niveaux... responsabilité de la prise de décision et de la gestion en matière financière. Elle réduit considérablement les coûts de transaction par rapport à d'autres procédures, lesquelles peuvent peser de façon excessive sur les capacités administratives des pays partenaires. Le décaissement rapide, trait spécifique de l'aide-programme, permet aussi de répondre plus facilement aux besoins de financement immédiats des partenaires lorsque la stabilité macro-économique, ainsi que la réalisation de programmes importants pour la lutte contre la pauvreté, risquent d'être compromises.

L'aide-programme suppose l'existence de conditions-cadres favorables dans les pays partenaires. Elle peut être utilement complétée par des projets précis ou des activités de coopération technique propres à renforcer l'aptitude des pouvoirs publics à réformer la gestion des affaires publiques, notamment à accrôttre la transparence, ainsi qu'à mettre en œuvre des programmes de lutte contre la pauvreté. Compte tenu du caractère fongible des ressources transférées au titre de l'aide-programme, il importe de faire en sorte que cette forme de coopération s'inscrive, comme il se doit, dans le cadre d'un programme de réforme défini d'un commun accord avec le FMI et la Banque mondiale. Tout en étant conscients du rôle particulier que jouent ces institutions dans l'apport de fonds et de conseils aux fins de la réforme économique, les organismes bilatéraux doivent veiller attentivement à ce que les objectifs de lutte contre la pauvreté gardent une place privilégiée dans la conception de tout programme de réforme. A cet égard, l'aide-programme peut créer des conditions propices à l'instauration d'un dialogue permanent sur les politiques favorables aux pauvres à mener dans les domaines décisifs que sont la réforme macro-économique et la bonne gestion des affaires publiques.

\section{Aide sectorielle}

Soucieux de l'efficacité des efforts de coopération pour le développement, beaucoup d'organismes de développement considèrent que l'aide à un secteur, sous la forme d'approches sectorielles, peut être un puissant moyen d'accroître l'appropriation locale, de renforcer le partenariat, d'assurer la coordination des ressources extérieures et intérieures, et de créer un contexte institutionnel propice au développement et au recul de la pauvreté.

L'approche sectorielle est un mécanisme permettant aux organismes d'aide et aux pays partenaires de soutenir le développement intégré d'un secteur donné. Ces organismes articulent leurs contributions dans un secteur autour d'une politique et d'un programme de dépenses uniques, arrêtés par le gouvernement du pays concerné, en s'efforçant d'appliquer autant que possible des méthodes de gestion et de notification communes pour le versement de tous les fonds et le contrôle de leur utilisation. Les approches sectorielles sont assez récentes et les problèmes qu'elles soulèvent au niveau de la réforme des institutions et de l'association des parties prenantes ne sont pas encore tous réglés. Elles peuvent devenir un moyen prometteur d'édifier des partenariats au service de la lutte contre la pauvreté, sous réserve que puissent être levées les contraintes existantes grâce à des initiatives visant à mettre en place les capacités institutionnelles nécessaires, faciliter la planification et la mise en œuvre tant au niveau national qu'à l'échelon local, et encourager l'appropriation des programmes sectoriels par les divers acteurs.

Les programmes sectoriels impliquent une approche de la gestion de l'aide qui diffère de celle à laquelle les organismes de développement sont accoutumés et pour laquelle ils sont organisés. Ils exigent que ces organismes tiennent pleinement compte des stratégies sectorielles locales. Ils obligent à modérer leurs ambitions et à accepter qu'un changement puisse prendre du temps, à œuvrer dans un esprit de construction de partenariats et non à s'en tenir à un schéma préétabli. L'octroi de cette forme d'aide dépend dans une large mesure de la situation du pays partenaire et est subordonné à un 
certain nombre de conditions préalables, notamment l'aptitude de ce pays à assumer les obligations liées à une bonne gestion financière.

Les nouvelles modalités de l'aide sectorielle n'en sont pour l'heure qu'à un stade embryonnaire. Afin de mettre à profit les avantages incontestables qu'offrent les programmes sectoriels du point de vue de l'efficacité de l'aide et de la lutte contre la pauvreté, les organismes de développement désireux de recourir à cette forme d'aide devront s'attacher à relever les défis suivants, mis en évidence par l'expérience limitée déjà acquise dans ce domaine :

Mettre l'accent sur l'objectif de réduction de la pauvreté. Les programmes sectoriels constituent un bon canal pour aborder les questions d'affectation des ressources et les liens entre équité et efficacité, d'une part, et lutte contre la pauvreté, d'autre part. En instaurant un dialogue sur les droits des pauvres et les problèmes d'égalité homme-femme qui se posent dans certains secteurs, il est possible d'influer sur les processus politiques et administratifs de manière à les rendre plus réceptifs aux besoins et aux souhaits des pauvres. Le fait que les fonds soient destinés à un secteur permet en outre, avantage non négligeable, d'internaliser des problèmes caractéristiques de l'aide-projet (comme le fait que des dépenses excessives soient consacrées aux hôpitaux régionaux au détriment des soins de santé primaires). Mais même dans les secteurs sociaux, il est souvent difficile de concilier une approche sectorielle avec un ciblage précis sur la réduction de la pauvreté en soi. Des mesures volontaristes sont parfois nécessaires pour faire en sorte que des progrès soient vraiment réalisés, au niveau non seulement de la qualité des services offerts mais aussi de leur facilité d'accès pour les pauvres et de l'amélioration du bien-être de ces derniers. Il peut se révéler indispensable de mettre au point des mécanismes de ciblage qui permettent de concentrer les services de façon disproportionnée sur les régions pauvres, les communautés défavorisées et les groupes vulnérables.

Faire une place de choix au renforcement des capacités. L'approche sectorielle suppose que tous les niveaux d'administration jouent un rôle plus actif dans la coordination de l'action des organismes de développement, l'élaboration des politiques, la fixation des priorités et l'exécution des programmes. La mise en œuvre des programmes sectoriels exige d'importantes réformes institutionnelles, notamment une redéfinition des rôles au sein de la hiérarchie politico-administrative et une réflexion sur les modalités de répartition des responsabilités entre les secteurs public, privé et communautaire. Les capacités du secteur public, du secteur privé et de la société civile ne sont pas toujours à la hauteur de programmes aussi complexes, ce qui peut nuire à la conception de la politique sectorielle et à l'ensemble du processus de mise en œuvre et de suivi des programmes. D'où la nécessité de prévoir de façon systématique, lors de la phase préparatoire des programmes sectoriels, des actions de renforcement des capacités et de promotion de la participation. Cela est indispensable pour assurer que la politique suivie sera en prise avec la réalité, que la plupart des acteurs concernés auront contribué à la définir et qu'ils seront en mesure de l'appliquer.

Faciliter la coordination avec les administrations locales. Si les administrations nationales assument davantage de responsabilités dans le cadre des programmes sectoriels locaux de l'administration, cela ne signifie pas pour autant que les acteurs intéressés aux échelons d'administration ou à l'extérieur du secteur public les font leurs. Étant donné que l'élaboration des programmes sectoriels est du ressort du pouvoir central et de ses ministères sectoriels, les administrations locales, qui tendent plutôt à suivre une démarche multisectorielle dans l'affectation des ressources et à être plus réceptives aux besoins locaux, risquent de perdre de leur influence. Les organismes de développement doivent appuyer la réalisation d'analyses visant à déterminer l'équilibre optimal à instaurer entre autorités nationales et locales, et les conditions requises pour améliorer la ....et à recentrer l'aide sur les besoins des populations pauvres.

Le soutien sectoriel implique qu'une place de choix soit faite au renforcement des capacités,...

... à la participation des administrations locales... 
...et à l'édification de partenariats entre secteur public et secteur privé. coordination entre les différents niveaux d'administration. Cet effort est particulièrement important dans le contexte de la décentralisation, dont le but est de confier davantage de pouvoirs aux collectivités locales. Les personnels chargés des activités de coopération technique qui font partie intégrante des programmes sectoriels peuvent se faire les apôtres de la participation à tous les niveaux, et servir d'intermédiaires entre les divers groupes d'intérêt, et contribuer ainsi à l'obtention de réels résultats sur le terrain.

Promouvoir le partenariat entre secteurs public et privé. Associer la société civile aux programmes sectoriels est une entreprise des plus ardues. Dans des secteurs productifs tels que l'agriculture ou les infrastructures, le processus de privatisation occupe une place importante, ce qui n'empêche que les programmes sectoriels sont généralement considérés comme des dispositifs qui relèvent du secteur public. Par conséquent, il importe de garder à l'esprit le principe selon lequel il faut s'appuyer sur la conjonction et la complémentarité des efforts de l'ensemble des acteurs concernés. Dans des secteurs sociaux comme la santé ou l'éducation, il est possible de renforcer la fonction de réglementation des ministères opérationnels, tout en encourageant la gestion décentralisée de la distribution des services, ce qui permettra aux prestataires privés (et organismes d'intérêt local) de jouer un rôle plus grand, ONG et secteur privé).

Rechercher des effets de synergie avec les politiques visant d'autres secteurs. L'une des principales raisons d'être des programmes sectoriels est de remédier au morcellement résultant du manque de coordination des investissements, en particulier des projets financés (et souvent gérés) par les donneurs, en inscrivant l'ensemble des activités concernant un secteur dans un cadre commun. Cette démarche permet d'améliorer sensiblement la coordination de ces activités en les articulant autour de stratégies sectorielles prises en main par le pays concerné. Cependant, en concentrant l'attention sur un seul secteur, on risque aussi de ne pas profiter des possibilités de coordination et de synergie avec les mesures prises dans d'autres secteurs. C'est une considération particulièrement importante dans la perspective de la lutte contre la pauvreté, car les pauvres ne vivent pas à l'intérieur de secteurs cloisonnés. La pauvreté déborde les limites sectorielles; il faut donc s'y attaquer en tenant compte de cette réalité. Il s'agit d'une véritable gageure, étant donné que la coordination entre ministères sectoriels est une tâche très complexe qui dérange des habitudes profondément enracinées et met à rude épreuve les capacités institutionnelles limitées dont disposent les différentes administrations.

Le souci de bonne pratique veut que priorité soit expressément donnée dans tous les programmes sectoriels aux besoins des pauvres. À cet égard, il est important de pouvoir s'appuyer sur des bilans de pauvreté (voir chapitre 1) qui fournissent des données tant quantitatives que qualitatives, ventilées par sexe, âge et catégorie sociale et tenant compte des aspects géographiques, culturels et socio-économiques. Le caractère pluridimensionnel de la pauvreté et des facteurs qui y contribuent peut ainsi constituer l'assise des programmes sectoriels, ce qui facilitera le raccordement de ces derniers à la stratégie générale de lutte contre la pauvreté du pays concerné. 


\section{Encadré 10. L'aide sectorielle au service de la lutte contre la pauvreté : le cas du secteur de l'éducation en Ouganda}

Le programme ougandais pour l'accès universel à l'enseignement primaire (Ugandan Universal Primary Education programme) constitue un bon exemple d'approche sectorielle dans le domaine de l'éducation. Il réunit les principaux ingrédients d'un partenariat axé sur la lutte contre la pauvreté.

- Ce programme a été lancé grâce à l'initiative énergique d'un chef d'État résolu à promouvoir l'accès universel à l'enseignement primaire.

- L'action à mener a été définie dans le cadre d'un processus largement fondé sur la participation, qui a comporté des consultations avec des groupes de la société civile et, à un stade ultérieur, des représentants des pauvres.

- Les crédits budgétaires affectés au secteur de l'éducation, qui ont été augmentés et intégrés dans le Poverty Action Fund, témoignaient de l'attachement des plus hautes sphères politiques à la réalisation de l'objectif visé.

- La conception du programme s'est directement appuyée sur une analyse approfondie de la pauvreté, reposant sur des enquêtes auprès des ménages et des bilans de pauvreté à caractère participatif, qui a servi à étayer la définition des priorités en matière de dépenses à travers le cadre budgétaire à moyen terme.

- La détermination avec laquelle le gouvernement a pris en main cette initiative a incité les organismes de développement qui attachaient la même importance à l'enseignement primaire à ne pas ménager leur soutien.
Ce programme offre aussi un modèle de bonnes pratiques pour le renforcement de la transparence dans la mise en œuvre. À cet égard, ses principales caractéristiques sont notamment les suivantes :

- L'analyse de la pauvreté et l'exercice du suivi ont été confiés au puissant ministère des Finances.

- Le parlement, la société civile et les organismes de développement ont été associés aussi bien à la prise des décisions qu'au suivi de la mise en œuvre du programme.

- L'accent a été mis sur la transparence, le but étant d'offrir aux utilisateurs des services éducatifs la possibilité de demander des comptes aux prestataires en mettant à leur disposition de multiples sources d'information et de nombreux circuits pour la formulation de suggestions visant à améliorer ces services.

- Des mesures ont été prises pour remédier au détournement des ressources (qui étaient orientées vers les écoles mais absorbées par les districts).

- Des efforts ont été faits pour renforcer le rôle des communautés villageoises dont des représentants ont été invités à siéger dans des commissions scolaires et se sont vu confier certaines responsabilités financières.
Harmoniser les méthodes comptables et les règles de notification. Les financements alloués à l'aide sectorielle peuvent transiter par des mécanismes parallèles (postes budgétaires) ou être déposés sur un fonds commun. Tout dépend si les partenaires sont parvenus à se mettre d'accord sur certains grands principes devant régir l'emploi du budget affecté au secteur en question. Les organismes de développement devront tôt ou tard définir des moyens d'alléger la charge qu'impose aux partenaires la multitude de procédures différentes et complexes fixées par les donneurs. Des efforts doivent être déployés pour simplifier jusqu'à un certain point et, dans la mesure du possible, harmoniser les procédures appliquées, sans que soit pour autant perdue de vue la nécessité de garantir la transparence et la reddition de comptes. A cet égard, le Groupe d'étude du CAD sur les pratiques des donneurs devrait fournir des orientations complémentaires de nature à renforcer l'appropriation locale en même temps qu'à réduire les coûts de transaction et les risques de détournement de l'aide, aussi bien pour les organismes de développement que pour les pays partenaires. Au niveau des gouvernements, cela impliquera la mise en place de systèmes transparents d'établissement du budget et de dispositifs indépendants de contrôle et de vérification des dépenses.

En résumé, les nombreux problèmes que soulèvent le renforcement des capacités institutionnelles, l'association des administrations locales, l'établissement de partenariats entre secteurs public et privé et l'amélioration de l'appropriation locale et de la transparence peuvent dans un premier temps détourner l'attention de l'objectif principal, à savoir faire reculer la pauvreté. L'expérience montre toutefois qu'il est possible de

\section{Le financement de l'aide sectorielle appelle une harmonisation des procédures de notification appliquées par les organismes d'aide parallèlement à une amélioration de la transparence et un renforcement des obligations de comptes dans les pays partenaires.}




\section{Encadré 11. Promouvoir des systèmes de santé favorables aux pauvres}

Assurer aux populations un bon état de santé est indispensable pour faire reculer la pauvreté et promouvoir le développement social et économique. De récents travaux d'analyse consacrés aux facteurs de la croissance ont en effet appelé l'attention sur le rôle que peuvent jouer dans ce domaine une amélioration de l'état de santé et un ralentissement de la progression démographique. Par conséquent, trois des objectifs de développement du millénaire définis pour 2015 portent plus spécifiquement sur les résultats en matière de santé: réduire des deux tiers la mortalité infantile et juvénile ; réduire des trois quarts la mortalité maternelle ; arrêter la propagation du VIH/sida et stabiliser le nombre de victimes du paludisme et d'autres grandes maladies, et inverser les tendances actuelles.

Pour atteindre ces objectifs, il est essentiel de donner la priorité à la santé des populations pauvres, gage de leur survie et de leur aptitude à subvenir à leurs besoins. Les pratiques qui suivent sont apparues de nature à accentuer les retombées des systèmes de santé sur la pauvreté :

- Rallier des énergies, nouer des partenariats et mobiliser des ressources au profit de systèmes de santé favorables aux pauvres. Mettre la santé au centre du dialogue sur les stratégies de lutte contre la pauvreté. Recenser les mesures qui, en dehors du secteur de la santé, sont susceptibles de contribuer à une amélioration de l'état sanitaire. Élargir le partenariat afin d'y associer la société civile et le secteur privé (comme cela a été fait pour l'éradication de la poliomyélite). Dans le droit fil de l'objectif 20/20, relever le niveau, actuellement très bas, de l'aide en faveur de la santé (environ $6 \%$ de l'APD) en vue de soutenir la mise en œuvre de programmes axés sur la pauvreté et pris en main par le pays partenaire dans le secteur de la santé.

- Faire en sorte que les systèmes de santé répondent mieux aux besoins des populations pauvres. Assurer des services de santé de qualité. Mettre en place des systèmes de tarification équitables et raisonnables. Apprendre au personnel de santé à respecter la dignité des pauvres. Tenir compte des raisons qui font que certaines personnes sont privées de soins : discrimination à l'égard des femmes, exclusion sociale, isolement géographique.

- Centrer les efforts sur les risques sanitaires qui perpétuent la pauvreté. Tourner l'attention vers les grandes maladies - paludisme, diarrhées, tuberculose - qui sont omniprésentes dans les populations pauvres. S'attaquer à la morbidité et la mortalité maternelles, à la pandémie du sida et aux maladies liées au tabagisme et à l'environnement. Il est nécessaire d'évaluer les risques sanitaires aussi bien au niveau local qu'au niveau national, en mettant en lumière les risques spécifiques auxquels sont exposés les hommes, les femmes et les enfants. Des données sur la santé ventilées par tranche de revenu, âge, sexe et localité doivent être complétées par des travaux de recherche à caractère participatif qui rendent compte des besoins exprimés par les pauvres dans le domaine de la santé.

En outre, une action internationale peut favoriser l'émergence de biens publics mondiaux dans le domaine de la santé (par exemple, un recentrage sur les maladies liées à la pauvreté et les vaccins correspondants).

\section{Une nouvelle génération d'approches territoriales pourrait s' attaquer aux multiples facettes de la pauvreté.}

parer à ce risque avec les programmes de la deuxième génération, dont le potentiel de réduction de la pauvreté a plus de chances de se concrétiser. Il est essentiel de mobiliser les membres expérimentés du personnel des organismes de développement présents sur le terrain pour conduire le dialogue sur les politiques à suivre en vue de faire reculer la pauvreté et pour aider à relever les défis évoqués plus haut.

L'intérêt porté à l'aide sectorielle permet de mieux circonscrire le dialogue sur les politiques et programmes en matière de développement social, concernant en particulier les secteurs de la santé et de l'éducation qui ont une importance déterminante pour la réduction de la pauvreté (encadrés 10 et 11).

\section{Approches territoriales}

Malgré l'inefficacité dont elles ont fait preuve dans le passé, les approches intégrées limitées à une zone rurale particulière ont récemment fait leur réapparition dans le contexte de la libéralisation politique et économique à l'œuvre dans les pays partenaires. Lorsque les autorités locales disposent d'une autonomie suffisante et que les marchés ruraux ont été libéralisés, il est possible de s'affranchir du cadre institutionnel hiérarchique qui a contribué à l'échec des programmes intégrés de développement rural. Le regain d'intérêt pour les approches territoriales s'explique aussi par le souhait des organismes d'aide de s'attaquer aux multiples facettes de la pauvreté et de mettre l'accent sur la durabilité des moyens d'existence ${ }^{2}$ en œuvrant dans plusieurs secteurs à la fois. 
Souvent centrées sur la production rurale (cultures, élevage, pêche, exploitation forestière, conservation des ressources), les approches territoriales visent à consolider les services de base tels que la santé, l'éducation et l'approvisionnement en eau, ainsi que les infrastructures (par exemple les routes rurales) et l'emploi dans les petites entreprises non agricoles. L'idée de départ est d'adopter une approche globale en tenant compte de la diversité des facteurs qui influent sur le revenu et le bien-être des populations pauvres. Cela suppose une bonne connaissance du contexte social, institutionnel et stratégique dans lequel s'inscrivent les activités dont les pauvres tirent leurs ressources, notamment la dynamique des relations entre les hommes et les femmes et le recours à des approches à caractère participatif pour réunir des informations. Un grand nombre de programmes mettent l'accent sur le renforcement des capacités tant des communautés que des administrations locales, créant dans certains cas un espace de négociation où les pauvres et leurs représentants peuvent faire entendre leur voix.

Si elles sont conduites par les pays partenaires et si elles sont viables sur le plan financier et institutionnel, les nouvelles approches territoriales peuvent contribuer à créer un environnement propice à la réduction de la pauvreté au niveau local. Se pose cependant un problème récurrent d'échelle et de durabilité dans la mesure où les efforts sont circonscrits à des zones où des projets sont financés par des ressources extérieures. Une grande attention doit être portée aux liens avec les pouvoirs publics et au respect des priorités nationales afin d'éviter le type de développement fermé qui a prévalu dans le passé. Fondamentalement, les réformes entreprises pour asseoir l'action des autorités locales sur des bases sûres et démocratiques sont déterminantes pour la réussite à long terme des activités de lutte contre la pauvreté fondées sur une approche territoriale. Cette question renvoie à celle plus générale de la décentralisation, notamment la délégation de responsabilités et de moyens financiers pour la fourniture de services de base, et de sa pertinence du point de vue de la lutte contre la pauvreté.

\section{Aide-projet}

Les projets sont depuis longtemps utilisés dans le cadre de la coopération pour le développement. Ils ont l'avantage de permettre de sélectionner un ensemble gérable de problèmes issus d'une réalité complexe, et de prescrire les moyens à mettre en œuvre pour stimuler le développement local. Cependant, l'expérience de nombreux organismes de développement montre que les projets isolés présentent de sérieuses limites dans le cas de certains pays partenaires. S'ils ne sont pas intégrés dans les cadres de planification nationaux, ils peuvent fausser la structure des dépenses nationales en utilisant des voies de financement distinctes et risquent d'imposer des coûts de gestion élevés. Ces projets isolés sont souvent gérés par les organismes d'aide selon une approche centralisatrice dans les phases de conception comme de mise en œuvre, ce qui entraîne des problèmes de viabilité après le retrait de l'aide extérieure.

C'est pourquoi les organismes de développement s'orientent vers des projets, susceptibles de donner des résultats plus durables et d'avoir une plus grande incidence sur la pauvreté. On s'applique désormais à concevoir les activités de telle sorte qu'elles s'insèrent dans un cadre de développement plus vaste, par exemple l'aide au niveau national ou les approches sectorielles. Des projets visant à renforcer les moyens d'action des pauvres, qui privilégient l'appropriation, la participation et la viabilité, commencent à voir le jour. Leur objet est d'accroître les capacités locales et de donner aux populations locales les moyens de s'organiser et de se mobiliser. Ils encouragent aussi les parties prenantes à jouer un rôle pilote dans la planification et la mise en œuvre des activités et dans la recherche des conditions assurant leur viabilité financière. La compatibilité du projet avec l'environnement institutionnel et culturel et son acceptation par les
Pour être viables, les projets doivent trouver leur place dans les cadres nationaux de planification ou dans une approche sectorielle. 


\section{Encadré 12. Axer les projets d'infrastructure sur la réduction de la pauvreté}

Pour s'assurer que les projets sont axés sur la réduction de la pauvreté, il convient de les analyser au regard de chacun des critères ci-après : techniques utilisées, zones ou régions choisies, priorités et organisation. Les exemples suivants sont tirés de projets d'infrastructure, lesquels ne présentent sans doute pas avec la réduction de la pauvreté des liens aussi évidents que des projets sociaux.

- Techniques utilisées : Les techniques à forte intensité de maind'œuvre sont souvent plus favorables aux pauvres que celles faisant une large place aux machines. Pour la construction de routes, elles sont souvent meilleur marché et permettent de tisser des liens importants avec l'économie locale. Il faut cependant veiller à ce que les femmes aient accès aux emplois ainsi créés car, lors du recrutement, des distinctions fondées sur le sexe peuvent être établies.

- Zones ou régions choisies : L'aide doit être dirigée vers des zones ou régions où les pauvres prédominent. Par exemple, l'électrification des zones rurales est plus favorable aux pauvres que l'électrification des zones urbaines, et l'amélioration des infrastructures dans les bidonvilles est davantage susceptible d'aider les populations pauvres que l'aménagement urbain général. Des arbitrages sont néanmoins parfois indispensables: dans le cas d'un investissement en équipements, le souci d'efficience dans l'utilisation des ressources peut conduire à favoriser des choix de nature à stimuler un développement rapide et à large assise. Tel sera par exemple le cas s'il existe une forte demande d'énergie de la part d'un secteur privé émergent qui est susceptible de créer des emplois et de générer des recettes fiscales mais qui n'est pas implanté dans les régions où vivent les populations pauvres.

- Priorités : Les routes secondaires et les routes de desserte sont souvent plus importantes pour les pauvres que les routes principales. Pour avoir accès aux marchés, les pauvres des zones rurales ont généralement besoin de routes de meilleure qualité et de moyens de transport plus réguliers. Étant donné que leur habitat est souvent dispersé, un réseau nourri de routes secondaires les intéresse davantage que des transports interurbains de haut de gamme, dont, par ailleurs, les pauvres des zones urbaines n'ont que faire. Dans certains cas, le financement des coûts récurrents peut revêtir une plus grande importance pour les pauvres que l'investissement initial. Cependant, les organismes d'aide ne sont souvent pas en mesure d'obtenir des pouvoirs publics l'engagement de prendre en charge les coûts récurrents liés à l'exploitation et à l'entretien de leurs projets d'infrastructure.

- Organisation: Dans les projets d'infrastructure, il est important de recenser les besoins et priorités locaux et d'en tenir compte. Si l'amélioration des infrastructures est souvent abordée sous un angle technique à l'échelon national, il est généralement admis que la participation des populations pauvres influe considérablement sur le fonctionnement à long terme et la viabilité de celles-ci. La prise en compte du point de vue des populations pauvres sur les programmes d'infrastructure peut contribuer à accroître l'impact de ces programmes sur la pauvreté.

autorités publiques locales et centrales ainsi que par la société civile sont deux facteurs de réussite fondamentaux.

La bonne pratique en matière de réduction de la pauvreté, exige des projets qui répondent aux multiples besoins des pauvres (revenu, dignité et sécurité, par exemple), tout en renforçant leur aptitude à s'assurer des moyens d'existence durables. Cela implique notamment de :

- Déterminer qui sont les pauvres et comment ils vivent, à l'aide de bilans de pauvreté et d'évaluations des besoins des bénéficiaires.

- S'inspirer de la connaissance qu'ont les populations pauvres de leur propre situation, ainsi que des actions et des stratégies de survie qu' elles mettent en œuvre, en appliquant des méthodes de type participatif afin de les associer aux projets.

- Faire de la problématique homme-femme une question de premier plan tout au long du cycle des projets, au lieu de lui réserver une place secondaire. 


\section{Aide centrée sur la pauvreté et interventions ciblées}

Viser les populations pauvres est une manière de s'assurer que les projets sont bien axés sur les actions et les stratégies des pauvres (par exemple les paysans sans terre, les habitants des taudis et les familles dirigées par des femmes). Pour apporter une aide ciblée, les organismes de développement ont recours à des instruments tels que les programmes intégrés de sécurité alimentaire et les programmes vivres contre travail, les fonds sociaux et le micro-crédit. Ces interventions au niveau micro-économique sont destinées à aider les communautés pauvres à se prémunir contre les risques (qu'ils soient structurels car dus à une pauvreté chronique ou transitoires car imputables à des chocs). Elles mettent généralement l'accent sur la mobilisation au niveau local, la participation, l'effort personnel et la capacité à se prendre en charge.

Les programmes vivres contre travail sont des interventions ciblées qui s'adressent essentiellement aux pauvres : ils n'attirent généralement pas les populations mieux loties car ils ne procurent que de maigres avantages. Le bilan est contrasté en ce qui concerne la durée de leur impact sur la pauvreté.

Les fonds sociaux sont des dons octroyés par les organismes de développement pour financer des petits projets gérés par des communautés pauvres. Ils visent à rendre autonomes les pauvres en les associant étroitement à la définition des priorités de développement (essentiellement dans les domaines de la santé, de l'éducation et de l'approvisionnement en eau), à la gestion des fonds consacrés aux projets, à l'entretien des équipements et au maintien des services une fois le projet terminé. Cependant, les fonds sociaux ont donné des résultats mitigés - ce type d'aide est difficile à inscrire dans la durée et soulève des problèmes lorsqu'il est mis au service d'objectifs politiques locaux plutôt que la lutte contre la pauvreté. Les activités financées font parfois fi des priorités budgétaires déjà approuvées dans un secteur donné (par exemple si les fonds sociaux servent à créer de nouveaux dispensaires non prévus dans le programme national concernant le secteur de la santé). Cette formule donne toutefois de bons résultats lorsqu'il est clairement établi que les fonds doivent être mis au service de la lutte contre la pauvreté, être centrés sur des activités à caractère purement local et compléter les activités lancées par les pouvoirs publics sans aller à l'encontre des priorités établies.

Les programmes de micro-crédit ont le plus souvent pour but de réduire la vulnérabilité des ménages pauvres en élargissant l'éventail de leurs sources de revenu possibles, en augmentant leur productivité et en favorisant l'accès des femmes à l'autonomie. Cependant, dans bien des cas, ils ne parviennent pas à toucher les populations qui vivent dans l'extrême pauvreté et ils induisent rarement à eux seuls un accroissement suffisant des revenus pour sortir les gens de la pauvreté. Leur impact est plus grand lorsqu'ils sont associés à une aide destinée à apprendre aux populations cibles comment fonctionnent les marchés, comment gérer une entreprise, comment rechercher des débouchés pour les produits fabriqués localement, et comment acheter et exploiter au mieux les terres. Les programmes visant à améliorer l'accès des pauvres aux ressources financières doivent aussi prendre en considération les différences entre les hommes et les femmes. Cela signifie qu'il faut trouver une solution au problème des garanties lorsque des obstacles juridiques ou culturels empêchent les femmes de détenir des titres de propriété foncière ou d'autres actifs. Cela signifie aussi qu'il faut expliquer aux femmes, en particulier si elles risquent d'être exclues des groupes de bénéficiaires ou de ne pas avoir le temps de déposer un dossier, comment accéder au crédit et comment l'utiliser. Les programmes de micro-crédit n'auront qu'une incidence limitée s'ils ne sont pas étayés par des mesures macro-économiques de nature à favoriser le développement des marchés financiers et l'essor d'institutions financières informelles viables. Dans les zones rurales, la plupart des systèmes publics de crédit ne sont pas conçus pour servir les pauvres, et
Les interventions

ciblées ont pour but de mobiliser les communautés pauvres en mettant l'accent sur la participation, l'effort personnel et l'autonomisation. 
Le renforcement des capacités doit constituer la toute première priorité de l'action engagée pour soutenir les efforts déployés par les partenaires pour formuler et mettre en œuvre une stratégie de lutte contre la pauvreté. les établissements bancaires du secteur privé, qui les remplacent de plus en plus, ne considèrent pas les pauvres comme un bon risque-crédit ou comme une clientèle rentable. Il faut donc mettre au point des stratégies de crédit spéciales à l'intention des pauvres.

Lorsqu'ils financent des petits projets ou des interventions ciblées, les organismes de développement doivent veiller à ce que des activités centrées sur un nombre restreint de pauvres et bénéficiant d'une grande visibilité ne détournent pas l'attention de programmes sectoriels et indirects tout aussi importants qui touchent un plus grand nombre de pauvres.

\section{Coopération technique à l'appui du renforcement des capacités}

La coopération technique (CT) est souvent une composante essentielle des instruments de coopération pour le développement évoqués ci-dessus. Elle a un rôle fondamental et durable à jouer dans la définition des stratégies de lutte contre la pauvreté et la mise en œuvre de programmes en faveur des pauvres. Qu'elle soit ou non associée à une coopération financière visant des programmes, secteurs ou projets particuliers, elle a pour but de renforcer les capacités institutionnelles et humaines des pays partenaires. Du fait qu'elle contribue à améliorer les cadres juridique et administratif, l'efficience des ressources humaines et le fonctionnement des organisations, la coopération technique concourt en outre de façon déterminante à accroître l'efficacité de la coopération pour le développement.

Le renforcement des capacités doit constituer la toute première priorité de l'action engagée pour soutenir les efforts entrepris par les partenaires pour mettre en place un environnement plus propice à la lutte contre la pauvreté. Bon nombre de pays partenaires ont besoin de doter leurs secteurs public, privé et non gouvernemental d'institutions efficaces et de ressources humaines qualifiées pour mettre en œuvre et pérenniser les réformes dans les vastes domaines si déterminants pour la réduction de la pauvreté que sont la politique macro-économique, la bonne gestion des affaires publiques et le développement social. Les organismes de développement peuvent concourir au renforcement des capacités nationales et locales nécessaires à l'élaboration des politiques, à la planification et à la gestion des programmes sectoriels, ainsi qu'à la fourniture de services sociaux de meilleure qualité, d'accès plus aisé, plus équitables et plus efficaces. En outre, le personnel de coopération technique peut faire fonction de médiateur dans les activités qui font appel à de multiples parties prenantes. La médiation revêt une importance particulière dans le contexte de la lutte contre la pauvreté, qui donne immanquablement lieu à des conflits d'intérêts entre différentes fractions de la collectivité.

Les actions en faveur du renforcement des capacités doivent aussi tenir compte de la progression galopante de la pandémie du sida dans beaucoup de pays pauvres. Ce sont les pauvres qui sont le plus durement touchés par cette maladie car celle-ci décime l'appareil administratif national au service de la lutte contre la pauvreté, prive les ménages pauvres de leurs apporteurs de revenu et accrôt le nombre de familles dirigées par des orphelins. Rehausser le caractère participatif des processus destinés à dégager un consensus sur les stratégies de réduction de la pauvreté nécessite aussi d'améliorer les capacités de la société civile, afin que celle-ci puisse participer plus efficacement à l'élaboration et à la mise en œuvre des politiques. Cela implique aussi de renforcer les capacités de groupes de défense d'intérêts particuliers qui aident les populations pauvres à gagner de l'influence en tant qu'interlocuteurs politiques. 
Dès lors qu'est assurée l'appropriation locale, les résultats de la coopération technique dépendent pour beaucoup de la détermination des organismes de développement à appliquer des approches ayant fait leurs preuves pour accroître l'efficacité et l'impact à long terme des programmes de $\mathrm{CT}^{3}$, à savoir notamment :

- Faire de l'autonomie un objectif stratégique en mettant l'accent sur le renforcement des capacités à long terme, plutôt que de chercher à améliorer les résultats à court terme en comblant les lacunes au niveau des compétences.

- Planifier les activités de coopération technique dans le cadre des stratégies de réduction de la pauvreté et des programmes sectoriels des pays partenaires au lieu de formuler des propositions isolées, inspirées par des considérations liées à l'offre.

- Définir les objectifs en termes de résultats à obtenir et non de ressources à mettre en œuvre.

- Encourager un recours plus grand aux compétences locales et déterminer le rôle précis que doivent jouer les spécialistes internationaux s'il y a lieu.

- S'appuyer sur les institutions et capacités existantes, tant publiques que privées, au lieu de promouvoir des structures parallèles.

- Utiliser des instruments de planification et de gestion à caractère participatif, et s'appuyer sur les mécanismes de réforme politique issus de l'initiative locale.

- Évaluer l'aptitude des pays partenaires à faire face aux coûts récurrents et à intégrer les coûts associés à la coopération technique dans le budget national.

Priorité doit être donnée aux activités de coopération technique susceptibles d'avoir des effets de grande portée en facilitant l'accès des pauvres aux services sociaux de base (éducation et santé) et - s'agissant des femmes et des groupes socialement marginalisés qui vivent de l'agriculture de subsistance et de la micro-entreprise - au crédit, à la technologie et au conseil. Avec la décentralisation, un rôle nouveau revient en outre à la coopération technique : renforcer les capacités des administrations locales, des municipalités et d'organismes privés tels que les établissements de crédit locaux et les associations professionnelles. La coopération technique sera par ailleurs appelée à jouer un rôle déterminant face aux défis que posera dans l'avenir la lutte contre la pauvreté, eu égard à la nécessité d'améliorer la gestion des affaires publiques, de promouvoir les mécanismes démocratiques, de protéger les droits de la personne, de faire respecter l'état de droit, ainsi que de renforcer les marchés et le développement du secteur privé.

\section{Instruments à finalité particulière}

Cette section porte sur l'utilisation de l'allégement de la dette, de l'aide humanitaire et du soutien de la coopération régionale, trois instruments qui occupent une place grandissante dans les portefeuilles des organismes d'aide et qui sont étroitement liés à la lutte contre la pauvreté.

\section{Allégement de la dette}

Dans les pays pauvres très endettés (PPTE), les paiements en devises fortes au titre du service de la dette détournent des ressources nationales déjà peu abondantes des investissements dans des domaines tels que l'éducation et la santé, qui sont essentiels pour faire reculer la pauvreté. L'allégement de la dette fait donc partie intégrante de la contribution de la communauté internationale à la lutte contre la pauvreté. A cet égard, divers mécanismes sont utilisés, qui vont de l'annulation pure et simple des créances à
La durabilité des

résultats de la coopération pour le développement est assujettie à l'application de diverses approches ayant fait leurs preuves.
L'allégement de la dette fait partie intégrante de la contribution de la communauté internationale à la lutte contre la pauvreté. 
Les efforts de coopération pour le développement doivent être centrés sur la réduction de la pauvreté et des inégalités à la fois en tant que causes et que conséquences des conflits. la conversion de la dette bilatérale en subventions à des fondations locales bailleuses de fonds, en passant par le rachat de créances à des fins écologiques.

Dans le cadre de l'Initiative renforcée en faveur des PPTE, les pays Membres de l'OCDE se sont engagés à contribuer à un allégement de la dette plus large et plus rapide ayant pour objectif exprès de dégager des fonds au service de la lutte contre la pauvreté. L'impact sur la pauvreté de cet effort sans précédent dépend, d'une part, des progrès accomplis par les PPTE dans l'élaboration et la mise en œuvre de stratégies de lutte contre la pauvreté et, d'autre part, de la disposition des pays de l'OCDE à dégager les financements requis sans réduire les autres apports d'aide. Afin de s'assurer que les fonds dégagés par les opérations d'allégement de la dette sont utilisés à bon escient, il convient de mettre en place des systèmes de suivi permettant un examen minutieux des dépenses budgétaires.

A l'avenir, les prêteurs tout comme les pays emprunteurs devront prendre des mesures pour éviter l' accumulation de nouvelles obligations, intenables, de remboursement. Pour compléter les annulations de dettes consenties dans le cadre de l'Initiative en faveur des PPTE, les organismes de développement doivent étudier d'autres possibilités d'allégement de la dette, par exemple les conversions de créances en programmes de renforcement des capacités dans des domaines où ceux-ci sont susceptibles d'avoir un fort impact sur la pauvreté. Dans le même temps, il importe de ne pas perdre de vue les besoins d'aide des pays ayant adopté des politiques judicieuses qui leur ont permis d'éviter de graves problèmes d'endettement.

\section{Aide en faveur de la prévention des conflits et aide humanitaire}

Les efforts de coopération pour le développement visant à prévenir les conflits violents ou à restaurer la paix doivent être centrés sur la réduction de la pauvreté et des inégalités à la fois en tant que causes et que conséquences des conflits. Dans le même temps, doit être apporté un soutien à long terme en vue de renforcer l'appareil gouvernemental et de bâtir des institutions et des mécanismes démocratiques propres à désamorcer les tensions sans explosion de violence. En cas d'extrême urgence, de conflit violent ou de catastrophe naturelle, l'aide humanitaire offre un moyen rapide de sauver des vies et d'atténuer les souffrances, souvent à grande échelle.

Dans la mesure où les populations pauvres n'ont pas les moyens d'échapper aux crises, une assistance de ce type est donc par définition axée sur la pauvreté. Elle peut cependant être préjudiciable à la réduction de la pauvreté en créant des dépendances et en diminuant l'aptitude des communautés touchées à résoudre les problèmes et à se prendre en charge. La bonne pratique à cet égard consiste notamment à planifier ce type d'aide de manière à :

Tenir compte des besoins à long terme des bénéficiaires et des stratégies de survie adoptées par les populations pauvres.

- S'appuyer systématiquement sur une analyse des sexospécificités.

- Ne pas compromettre la production et la prestation de services au niveau local en inondant les marchés locaux de produits alimentaires.

- Renforcer le plus rapidement possible les capacités locales en vue de reprendre la gestion des opérations de secours ${ }^{4}$.

L'une des principales difficultés de l'aide humanitaire est d'intégrer les activités de secours et de remise en état dans une action à plus long terme destinée à promouvoir l'autonomie. 


\section{Soutien de la coopération régionale}

L'élaboration d'une stratégie-pays visant à faire reculer la pauvreté doit s'inscrire dans une optique tant régionale que nationale. Dans le cas de beaucoup de pays partenaires, l'adoption d'un point de vue et de solutions de portée régionale s'impose pour transcender les limites de l'économie nationale avec son marché de taille restreinte et sa faible dotation en ressources. L'accès aux marchés régionaux est source d'accélération de la croissance car vecteur d'amélioration de la compétitivité et de la productivité, et constitue donc un bon tremplin pour l'intégration dans l'économie mondiale. La coopération régionale peut faciliter la coordination des mesures de réforme économique et l'harmonisation des réglementations, conditions essentielles pour accroître les possibilités d'investissement et stimuler la croissance. Les avantages de l'intégration régionale commencent à devenir visibles, et les échanges intrarégionaux au sein de l'Asie, de l'Afrique et de l'Amérique latine se sont intensifiés au cours des années 90 , où ils ont progressé beaucoup plus vite que les échanges avec les pays de l'OCDE.

Les stratégies de survie des pauvres s'inscrivent souvent dans une perspective régionale, conduisant un ou plusieurs membres de la famille à émigrer vers des pays voisins où ils pensent avoir plus de chances de trouver une activité génératrice de revenus. Ces travailleurs migrants représentent une importante source de revenu pour leur famille à laquelle ils envoient leur salaire, et rapporteront ensuite chez eux des idées et des compétences nouvelles qui contribueront elles-mêmes à la création d'activités rémunératrices.

Parallèlement, il faut rechercher des solutions aux problèmes régionaux qui ont une incidence sur la pauvreté. Par exemple, la productivité du travail et les moyens d'existence des familles pauvres sont gravement affectés par des maladies comme le sida qui ignorent les frontières nationales, ou par la dégradation de ressources naturelles communes comme les bassins fluviaux, l'érosion des sols et la désertification.

Les organismes de développement doivent tenir compte de la dimension régionale de la lutte contre la pauvreté dans leurs stratégies-pays. En particulier, ils doivent s'interroger sur la façon dont les stratégies de lutte contre la pauvreté des pays partenaires pourraient tirer parti des institutions, des capacités et des marchés régionaux. Les centres d'excellence existant à l'échelon infrarégional peuvent contribuer à réduire le coût de la formation et de la recherche grâce à d'importantes économies d'échelle. Ces centres et les réseaux régionaux de spécialistes peuvent jouer un grand rôle dans la réalisation, sous la conduite des pays partenaires, des analyses de la pauvreté nécessaires pour éclairer les choix stratégiques des pouvoirs publics. Ils peuvent aussi contribuer à l'évaluation des résultats de la lutte contre la pauvreté, en même temps qu'au renforcement des capacités nationales requises pour la mise en œuvre des stratégies définies dans ce domaine.

\section{Conclusion}

La réduction de la pauvreté doit être au centre du dialogue sur les politiques à suivre et constituer le point d'ancrage de la planification et de la mise en œuvre de la coopération pour le développement. Dès lors que le contexte politique est favorable dans les pays partenaires, les activités de coopération pour le développement en faveur des pauvres doivent prendre en compte les éléments suivants :

La capacité de comprendre le problème de la pauvreté et de le relier à la gestion des affaires publiques et à la politique économique et sociale - en particulier en ce qui concerne les disparités entre hommes et femmes et les divers dispositifs institutionnels qui déterminent les droits.

La réduction de la pauvreté doit être au centre du dialogue sur les politiques à suivre et constituer le point d'ancrage de la planification et de la mise en œuvre de la coopération pour le développement. 
L'ampleur des ressources consacrées aux pauvres dans chaque secteur, ainsi que la facilité d'accès et la qualité des services publics. Il faut donc ménager une marge suffisante dans les budgets nationaux par une utilisation optimale des ressources nationales et de l'allégement de la dette.

- La détermination politique et le zèle de l'appareil administratif à mettre en œuvre les réformes proposées et à en observer les résultats. 


\section{Notes}

1. Voir les stratégies de développement durable, OCDE, Paris, 2001.

2. Voir le chapitre 1, pour une définition de la notion de moyens d'existence durables.

3. Voir les « Principes du CAD relatifs aux orientations nouvelles de la coopération technique » présentés dans les Principes du CAD pour une aide efficace, OCDE, Paris, 1992.

4. Voir les Lignes directrices du CAD sur les conflits, la paix et la coopération pour le développement (1998). Voir aussi Prévenir les conflits violents : quels moyens d'action? 


\section{Pour une cohérence des politiques à l'appui de la réduction de la pauvreté}

\section{Introduction}

Comme l'ont clairement démontré les chapitres précédents, pour être efficaces, les politiques de coopération pour le développement doivent être totalement compatibles avec l'objectif de lutte contre la pauvreté dans les pays partenaires, et accorder à ce dernier une place centrale. Cependant, la cohérence globale entre les politiques menées par les gouvernements des différents pays Membres de l'OCDE au regard de leur impact sur la réduction de la pauvreté à l'échelle mondiale est au moins aussi importante.

Les notions de cohérence, de cohésion et de coordination des politiques sont étroitement imbriquées. Pour aider à en distinguer les nuances, des définitions en sont proposées dans l'encadré 13 .

Assurer la cohérence des politiques au service de la lutte contre la pauvreté est une entreprise complexe du fait que les problèmes à régler font habituellement intervenir au niveau national des groupes d'intérêts et des organismes gouvernementaux dont les préoccupations essentielles et les responsabilités sont autres que la réduction de la pauvreté dans le monde. Il peut y avoir conflit, par exemple, entre les efforts que déploie un gouvernement pour répondre aux préoccupations de groupes spécifiques ou de communautés locales et son engagement à réduire la pauvreté dans les pays en développement. La cohésion des politiques tend à faiblir quand le caractère politiquement sensible de certaines questions et des intérêts qui y sont associés devient plus marqué, et à s'affirmer au contraire lorsqu'il existe dans le pays concerné une forte mobilisation pour combattre et tenter d'éliminer la pauvreté dans les pays pauvres.

Les conflits d'intérêts et d'objectifs, qui peuvent être encore accentués par le cloisonnement de la politique et de l'administration publique, ne peuvent se résoudre que par une coordination des politiques, qui est donc un processus tout autant politique qu'administratif. Les organismes de développement se trouvent souvent en position de faiblesse, sur le plan politique, par rapport aux autres entités gouvernementales et aux intérêts, publics et privés, prévalant dans des domaines tels que les échanges, l'investissement, l' agriculture et la sécurité nationale. Pourtant, plusieurs raisons militent aujourd'hui pour que la réduction de la pauvreté prenne davantage le pas sur d'autres objectifs nationaux, et s'élève dans l'échelle des priorités de tout un éventail de décideurs :

- Les dirigeants de ce monde et des institutions comme le G7/8, le FMI, la Banque mondiale, l'OCDE et les Nations unies ont adopté des déclarations d'orientation dans lesquelles ils font de la lutte contre la pauvreté un cadre de référence commun et un objectif stratégique majeur.

La pauvreté est de plus en plus perçue comme un «mal public planétaire », non seulement en vertu de considérations morales, mais aussi parce qu'elle est source de dysfonctionnements et de désordres ayant des retombées négatives à l'échelle mondiale, telles que l'instabilité politique, la dégradation de l'environnement, les flux migratoires, etc.
La coordination, la

cohésion et la

cohérence de toutes les politiques ayant des répercussions sur la pauvreté dans le monde revêtent une importance primordiale...

....et la conciliation de cet impératif avec les autres objectifs ralliant un ferme soutien politique au plan intérieur constitue un défi majeur...

...mais, pour diverses raisons, la lutte contre la pauvreté s'élève de plus en plus dans l'échelle des priorités des gouvernants et des décideurs,... 


\section{Encadré 13. Les différents aspects de la cohérence des politiques}

La cohérence appelle la satisfaction, simultanée qui plus est, d'un certain nombre de conditions, encore qu'il faille bien admettre qu'une totale cohérence ne sera en pratique jamais atteinte.

- La coordination des politiques implique l'instauration d'une collaboration entre les diverses structures institutionnelles et de gestion intervenant dans la formulation des politiques.

- La cohésion des politiques nécessite de faire en sorte que les politiques suivies dans un même domaine n'aient pas des effets opposés et d'éviter toute action qui irait à l'encontre de la réalisation d'un objectif donné, en l'occurrence la réduction de la pauvreté à l'échelle internationale.

- La cohérence des politiques va plus loin. Elle passe par la promotion systématique d'actions se renforçant mutuellement entre les divers domaines d'intervention des différents ministères et instances gouvernementales afin de créer des synergies au service de la réalisation de l'objectif poursuivi. ...ce qui facilite I'amélioration de la cohérence des politiques avec les immenses bienfaits qu' on peut en escompter en termes de réduction de la pauvreté.
Il s'opère une prise de conscience grandissante du fait que la zone OCDE ne compte plus aujourd'hui qu'à peine 1 milliard d'habitants, sur un total mondial de 6 milliards. Cette tendance ne pourra que s'accentuer sous l'effet de la pression démographique, sachant que $97 \%$ des 2 milliards d'individus supplémentaires qui, d'après les estimations, viendront au monde au cours des vingt prochaines années naîtront dans les pays en développement. Par conséquent, le bien-être de l'humanité tout entière passe de plus en plus par le règlement des problèmes de développement des pays pauvres.

Les effets d'une amélioration de la cohérence des politiques menées par les pays de l'OCDE sur les pays en développement pourraient être immenses. Les estimations établies par les Secrétariats de l'OCDE et de la Banque mondiale indiquent que les droits de douane et les subventions qu' appliquent les pays de l'OCDE aux produits agricoles et manufacturés entraînent probablement chaque année pour les pays en développement un manque à gagner d'un montant équivalant au total de leurs recettes d'APD. Si l'on y ajoute l'impact des obstacles non tarifaires, de la protection des services et d'autres mesures commerciales induisant des coûts de transfert et de transaction, au total, le coût des mesures de protection des pays de l'OCDE pour les pays en développement pourrait être plus de trois fois supérieur au montant de l'APD, et leur effet sur longue période beaucoup plus lourd encore. L'application des politiques agricoles coûte à elle seule aux pays de l'OCDE environ $\$ 1$ milliard par jour, soit près de sept fois le montant des apports d'APD consentis par les pays Membres du CAD.

Il est impératif, pour la réalisation des objectifs de développement du millénaire, que les Membres progressent encore sur la voie de la cohérence des politiques. Dans son document d'orientation stratégique intitulé Le rôle de la coopération pour le développement à l'aube $d u X X I^{e}$ siècle, le CAD rappelait que " notre action doit commencer par l'adéquation de l'éventail des politiques des pays industrialisés et des objectifs du développement. » 
En 2000, le Conseil de l'OCDE réuni au niveau des ministres et le CAD réuni à haut niveau ont décidé d'établir une liste des conditions essentielles à la cohérence des politiques qui pourrait servir de référence pour l'élaboration des politiques publiques des pays Membres. Une telle liste est incluse dans ces Lignes directrices (voir l'annexe au présent chapitre). Elle couvre tout un éventail de domaines ayant des retombées sur le développement. Elle se veut indicative plutôt qu'impérative et vise à encourager les gouvernements Membres à intégrer systématiquement les questions de développement et de pauvreté dans toutes leurs décisions. Cette liste a été élaborée à la suite de consultations entre experts spécialisés, au sein de l'OCDE, dans les divers domaines d'intervention des pouvoirs publics. Elle doit constituer un petit document distinct que pourront utiliser les différentes sphères de l'administration des pays Membres, et elle servira aussi à faciliter et guider les travaux ultérieurs de l'OCDE elle-même sur les questions de cohérence des politiques.

\section{Mondialisation et cohérence des politiques}

Il faut placer la cohérence des politiques dans le contexte global de la mondialisation. L'augmentation et l'accélération de la circulation des personnes, des biens, des services, des capitaux, de la technologie et de l'information hors des frontières nationales ont donné naissance à une économie mondiale de plus en plus intégrée. C'est l'une des marques essentielles de la mondialisation, qui a jeté les bases d'un univers où l'interconnexion et l'interdépendance n'ont jamais été aussi fortes. L'ouverture et l'intégration grandissantes de l'économie mondiale créent des conditions qui pourraient rendre possible une réduction massive de la pauvreté dans le monde. La mondialisation, génératrice d'immenses richesses, pourrait se révéler bénéfique au plus grand nombre. Encore fautil, pour cela, que la communauté internationale et les gouvernements prennent des mesures spécifiques à cet effet.

Les technologies de l'information et des communications (TIC) dont l'évolution est constante transforment les marchés, notamment les marchés financiers, et appellent de nouvelles méthodes d'organisation du travail, des entreprises et des échanges pour récolter les bienfaits de la mondialisation. Parallèlement, nombreux sont ceux qui s'inquiètent des mutations économiques et technologiques rapides en cours. Les ministres des pays de l'OCDE ont d'ailleurs reconnu qu'il était important de répondre à ces craintes, aux niveaux local, national et international.

La mondialisation s'accompagne de nouvelles opportunités, mais aussi de nouveaux défis pour tous les pays, toutes les régions, toutes les sociétés et tous les peuples. Si les exportations mondiales de biens et de services ont progressé à un rythme sans précédent depuis le début des années 80, la part des pays en développement n'y est que de $18 \%$ (alors qu'ils représentent $78 \%$ de la population mondiale). En dépit de leur accès préférentiel aux marchés, la part des pays les moins avancés (PMA) est tombée à $0.5 \%$ en 1999 (pour $11 \%$ de la population mondiale).

Les pays les plus pauvres ne seront pas à même de tirer des avantages significatifs des nouvelles opportunités commerciales si ne sont pas prises des mesures spéciales pour favoriser leur développement économique et social et le renforcement de leurs capacités institutionnelles. De même, les régions et groupes les plus pauvres de tous les pays sont désavantagés dans l'univers de plus en plus concurrentiel créé par la mondialisation économique et technologique.
La mondialisation accentue I'interdépendance et accroît l'intégration des marchés...

...sous l'effet de changements économiques et technologiques rapides qui ouvrent d'immenses possibilités de création de richesse... 
... mais suscitent aussi des craintes ; la principale difficulté consiste à faire en sorte que les pauvres parviennent à tirer profit de la concurrence grandissante qui caractérise l'économie mondiale...

... grâce à un renforcement, à cet effet, de la cohérence des politiques des organisations internationales ainsi que des gouvernements des pays en développement comme de ceux de I'OCDE.

\section{La cohérence des politiques nécessite une solide analyse, fondée sur l'observation des faits, des liens des diverses politiques avec la pauvreté et de leur impact sur cette dernière...}

...pour étayer la coordination des politiques et les négociations politiques, activités aux quelles il faut associer la société civile ;...
Dans l'optique de la lutte contre la pauvreté, la principale difficulté consiste à faire en sorte que les pays en développement et, en particulier, les pauvres dans ces pays, bénéficient de la mondialisation et soient capables de s'adapter avec succès à cette nouvelle donne. A cet effet, il est primordial d'assurer l'adhésion et la participation des pays en développement aux systèmes internationaux et aux cadres de coopération dans lesquels s'inscrit tout un éventail de politiques économiques et sociales, ce qui signifie qu'il faut prendre en considération leurs préoccupations et leurs points de vue et les aider à acquérir la capacité de prendre part, en tant que partenaires à part entière, aux négociations internationales concernant les domaines en rapport avec le développement.

C'est aux gouvernements des pays en développement qu'il incombe au premier chef de relever ce défi, mais il n'en reste pas moins qu'il est essentiel de rehausser la cohérence entre les politiques des pays de l'OCDE pour permettre aux pays en développement de tirer profit de la mondialisation et de faire reculer la pauvreté. Face à la mondialisation, les gouvernements des pays de l'OCDE doivent, plus que jamais, tenir compte des objectifs globaux de développement dans toutes leurs politiques et relations extérieures.

Dans un monde où la distinction entre les affaires nationales et internationales n'est plus tranchée, des programmes même efficaces de coopération pour le développement ne peuvent à eux seuls réduire la pauvreté autant qu'il le faudrait . Les objectifs de développement doivent être intégrés dans les activités de toutes les entités gouvernementales, non seulement des pays en développement mais aussi des pays de l'OCDE. Les moyens à mettre en œuvre pour y parvenir et les enseignements tirés de l'expérience acquise dans ce domaine font l'objet des deux sections suivantes. Les principaux domaines d'action qui gagneraient à une plus grande cohérence des politiques sont présentés ensuite et récapitulés dans l'encadré 16 . Une liste plus complète de ces domaines est présentée dans la Liste indicative figurant en annexe à ce chapitre.

\section{Moyens à mettre en œuvre pour améliorer la cohérence des politiques}

Améliorer la cohérence des politiques nécessite de connaître les mécanismes qui relient l'action engagée dans chaque domaine à la pauvreté dans les pays en développement. On dispose aujourd'hui de nombreuses données montrant en quoi les politiques menées dans divers domaines peuvent faire obstacle à la réduction de la pauvreté.

Il importe d'analyser soigneusement le rôle de chaque politique, son impact sur la pauvreté et à quel niveau, au sein du gouvernement, des ajustements peuvent être apportés de façon à renforcer la cohérence. Ce genre d'analyse nécessite des moyens appropriés. Les résultats doivent en être communiqués aux décideurs qui participent à la coordination interministérielle et aux négociations internationales. La cohérence des politiques ne sera peut-être jamais parfaite, mais il y a des domaines où il est relativement aisé de la renforcer.

L'ajustement des politiques au service d'une plus grande cohérence doit vraisemblablement s'inscrire dans un processus politique. Il est essentiel que les différents décideurs et acteurs aient en main tous les éléments nécessaires pour le justifier. A cet égard, les ONG et les médias peuvent jouer un rôle important en informant le public, mais il faut qu'ils disposent d'informations fiables, fondées sur des faits et sur une analyse solide. 
L'inégalité des chances économiques entre hommes et femmes est souvent aggravée par le manque de cohérence des politiques. C'est ainsi que les personnes qui ont le plus à souffrir, en tant que producteurs et que consommateurs, de la mondialisation sont souvent des femmes. Leur situation au regard de l'accès aux ressources, à l'information et aux technologies permettant d'exploiter les opportunités offertes par la mondialisation est déterminante. Il est donc capital d'intégrer aux processus de formulation des politiques et d'évaluation de leur impact une analyse des sexospécificités. Cette analyse peut faciliter à la fois la compréhension du phénomène de la pauvreté et la recherche de solutions et de mesures efficaces. La prise en compte des disparités entre hommes et femmes dès le début du processus d'élaboration d'une politique contribuera à renforcer les effets positifs et à éviter ou atténuer ses conséquences négatives.

Le souci de cohérence des politiques doit inciter les gouvernements des pays de l'OCDE à encourager et soutenir la participation des pays en développement à diverses instances internationales telles que l'Organisation mondiale du commerce, le Forum pour la stabilité financière et la Convention sur l'aide alimentaire. Il faut ouvrir aux nations en développement non seulement les séances formelles que tiennent ces instances, mais aussi les réunions informelles, si importantes pour l'aboutissement des négociations. La cohérence des politiques implique en outre que les délibérations, y compris celles des institutions financières internationales, soient transparentes et menées de façon responsable.

La capacité des pays en développement de participer à la négociation et à la mise en œuvre des conventions internationales, et à en tirer profit, a besoin d'être nettement renforcée. Plusieurs Membres du CAD proposent des programmes de renforcement des capacités dans le domaine du commerce international - visant, d'une part, à rehausser les aptitude à la négociation des fonctionnaires et, d'autre part, à améliorer la capacité du secteur privé à soutenir la concurrence sur les marchés mondiaux. Or, l'amélioration des capacités d'exportation des pays en développement risque d'accroître la concurrence pour les entreprises des pays de l'OCDE. Si les exportations des pays en développement devaient se heurter à des réactions protectionnistes visant à défendre des positions acquises sur des marchés potentiels, il faudrait y voir un test important pour la cohérence des politiques.

\section{Les enseignements de l'expérience des pays de l'OCDE}

Les gouvernements de plusieurs pays de l'OCDE s'appliquent depuis longtemps à renforcer la cohérence de leurs politiques au service de la lutte contre la pauvreté. Il se dégage de leur expérience plusieurs conclusions importantes. L'une des principales est que des politiques cohérentes permettent d'accroître sensiblement l'efficacité des efforts de réduction de la pauvreté. La cohérence rehausse en outre la crédibilité et la légitimité de l'action des gouvernements des pays de l'OCDE aux yeux de leurs partenaires.

Les réunions au sommet comme l'Assemblée du Millénaire des Nations unies et les réunions annuelles du G8 ont montré à quel point il importe que l'appel à la réduction de la pauvreté vienne d'en haut. Une fois cet impératif clairement énoncé, il devient beaucoup plus facile de renforcer les dispositifs qui permettront la conception de politiques cohérentes face aux forces centrifuges que sont les intérêts sectoriels et particuliers.

La cohérence des politiques des pays de l'OCDE rehausse I'efficacité de leurs efforts de lutte contre la pauvreté et leur crédibilité aux yeux des pays partenaires, surtout si le message vient d'en haut... 
...et est renforcé par des consultations avec des organismes publics et des acteurs privés dans le cadre d'une instance centrale de coordination et de contacts informels. Les pays Membres de l'OCDE $s^{\prime}$ appliquent à œuvrer dans ce sens, chacun à sa manière.
La cohérence des politiques exige que soient consultés des organismes publics et des parties prenantes privées. Il s'est révélé utile à cet égard de désigner une instance de coordination investie de l'autorité voulue pour mener à bien sa mission, de préférence haut placée dans l'exécutif, dont le rôle sera de tisser des liens solides entre un grand nombre de ministères et entités gouvernementales, ainsi qu'avec la société civile. Les rencontres formelles sont certes un instrument sans pareil au service de l'amélioration de la cohérence, mais l'instauration d'une culture organisationnelle propice au développement des contacts informels est également indispensable.

L'affirmation claire par le gouvernement de son objectif de lutte contre la pauvreté contribue directement à renforcer la cohérence des politiques. Ainsi, le Royaume-Uni a publié ces dernières années deux livres blancs sur l'éradication de la pauvreté dans le monde, dans lesquels il fait clairement siens les principes du partenariat et de la cohérence des politiques dans un large éventail de domaines d'intervention des pouvoirs publics. Des procédures formelles ont été mises au point pour assurer au sein du gouvernement les liaisons nécessaires à la cohérence des politiques. En outre, le Secrétaire au développement international a été élevé au rang de membre du Cabinet, ce qui a du même coup fait remonter l'objectif de la lutte contre la pauvreté dans l'échelle des priorités nationales (voir encadré 15).

Les États-Unis ont de plus en plus recours à des groupes de travail inter-organisations pour définir leurs stratégies internationales et étudier les problèmes de cohérence des politiques et les arbitrages à opérer. C'est dans ce contexte que tout est mis en œuvre pour parvenir à des décisions consensuelles prenant en considération les enjeux du développement. Les exemples illustrant cette démarche sont légion. On peut notamment citer le Committee on International Science, Engineering and Technology, qui formule des recommandations à l'intention de l'exécutif ; le Groupe de travail inter-organisations sur l'Initiative pour les pays pauvres très endettés, piloté par le Département du Trésor, qui a fait des questions de réduction de la pauvreté un volet à part entière de son mandat à l'égard des pays pauvres très endettés ; et l'Enterprise for the Americas Foundation, qui s'occupe de convertir des titres de créance en dons investis en faveur du développement local et est co-dirigée par le Département du Trésor, le Département d'État et l'USAID.

Aux Pays-Bas, le ministère des Affaires étrangères s'occupe de tous les aspects intéressant les relations entre les Pays-Bas et les pays en développement. L'objectif de ce décloisonnement est de susciter des synergies entre les différents secteurs de l'administration néerlandaise, de façon à renforcer la cohérence et l'efficacité globales de l'action menée. Le Département des affaires bilatérales du ministère des affaires étrangères veille à intégrer la coopération pour le développement et tous les autres aspects de la politique étrangère et des relations commerciales. Un Conseil des affaires européennes et internationales, créé par le Cabinet, est en outre chargé de veiller à la cohérence des politiques.

Dans ses Lignes directrices Nord-Sud, la Suisse insiste beaucoup sur la nécessité d'assurer la cohérence des diverses politiques ayant une incidence sur les pays en développement. Ce document a contribué à sensibiliser les ministères aux problèmes de cohérence des politiques. Des procédures formelles de consultation aux niveaux des cabinets et des ministères ont été instituées afin de repérer les incohérences éventuelles et, dans la mesure du possible, de les corriger. Une commission consultative, composée de représentants de larges pans de la société civile, est chargée de conseiller le gouvernement sur les questions de coopération pour le développement et joue également le rôle de mécanisme institutionnel de renforcement de la cohérence des politiques. Les ONG et les organisations politiques sont régulièrement consultées. 
Le Royaume-Uni a pris des dispositions radicales pour promouvoir la cohérence des politiques. Les principales sont les suivantes :

- Le gouvernement a clairement manifesté sa volonté de veiller à la cohérence des politiques. Le Département du développement international (DFID) est devenu autonome, et le Secrétaire d'État qui le dirige a désormais rang de membre à part entière du Cabinet. A l'issue de discussions approfondies entre les différents services gouvernementaux, un Livre blanc a été établi, puis présenté au Parlement et largement diffusé.

- Des moyens ont été dégagés pour assurer la cohérence des politiques. Le DFID s'est vu doter de ressources nouvelles pour pouvoir procéder à des analyses des retombées pour le développement des questions d'échanges et d'investissement, et participer au débat auquel celles-ci donnent lieu au sein du gouvernement. Il a également commandité des études sur la libéralisation du commerce agricole, la sécurité alimentaire et la biodiversité.
- Les mécanismes de coordination entre les différents ministères ont été renforcés. Un groupe de travail interministériel sur le développement a notamment été créé pour traiter des questions transversales. Le DFID a été représenté au groupe officiel qui s'est réuni régulièrement, sous la présidence du ministère du Commerce et de l'Industrie, pour débattre de la stratégie du Royaume-Uni dans les négociations commerciales multilatérales.

- Le DFID a resserré ses liens avec les organisations multilatérales, notamment l'OMC, la CNUCED, le CCI et la Banque mondiale qui s'occupent de domaines où la cohérence des politiques est capitale.

- Le DFID a aidé des pays en développement à renforcer leurs capacités afin de les mettre mieux à même de se préparer et de participer aux négociations internationales concernant les échanges, l'investissement et d'autres questions importantes.

En Allemagne, une réglementation prévoit l'examen systématique de toute loi nouvelle par le ministère de la Coopération pour le Développement (BMZ), afin d'en assurer la cohérence avec la politique en matière de développement. En outre, le BMZ siège au Conseil fédéral de sécurité, ce qui facilite la convergence entre les mesures de prévention des crises et de règlement des conflits et la politique en faveur du développement. L'Agence canadienne de développement international se concerte souvent directement avec d'autres services gouvernementaux à propos des mesures à prendre dans des domaines autres que la politique d'aide, concernant l'allégement de la dette, les préférences tarifaires à l'importation et d'autres sujets encore. Le gouvernement néo-zélandais s'est expressément engagé à assurer la cohésion et la cohérence de ses politiques. La Suède, pour sa part, a créé une commission parlementaire chargée de veiller au renforcement de la cohérence des politiques au service de la lutte contre la pauvreté dans le contexte de la mondialisation. La Norvège élabore actuellement un Plan d'action contre la pauvreté qui donne sa place à la cohérence des politiques. Quant à la Finlande, elle met l'accent sur la lutte contre la pauvreté et la sécurité collective dans sa stratégie intégrée concernant les relations avec les pays en développement.

Compte tenu de leur complexité, les structures de décision de l'Union européenne nécessitent le déploiement de ressources humaines considérables. Outre une coordination entre les différentes politiques de la Commission européenne (CE), il faut aussi assurer la coordination de ces dernières avec celles des États membres. Une fois trouvées des positions communes acceptables, il devient d'autant plus facile d' améliorer la cohérence. En témoignent, par exemple, les propositions de réforme relatives à la dissociation de la politique de sécurité alimentaire, y compris l'aide alimentaire, du problème de la liquidation des excédents agricoles de l'Europe. Ou encore l'Initiative « Tout sauf les armes », qui supprime les restrictions aux importations en provenance des pays les moins avancés. 
La communauté internationale affiche sa conviction qu' un réel recul de la pauvreté passe par l'association des femmes aux processus de consultation,...

...l'affectation de l'aide sur la base de critères de pauvreté...

... et le renforcement des capacités institutionnelles dans les domaines des échanges...

...et de la gestion de la dette.
L'une des conditions premières de la cohérence des politiques est d'élargir les processus de décision de façon à recueillir l'avis des femmes aussi bien que des hommes, pauvres en particulier, sur ce qui est important et sur les besoins qu'ils jugent prioritaires. A la Conférence mondiale des Nations unies sur les femmes qui s'est tenue à Pékin en 1995, il a été convenu que « les gouvernements et les autres acteurs devraient systématiquement et ostensiblement tenir compte des intérêts des femmes dans toutes leurs politiques et dans tous leurs programmes ». L'analyse des politiques doit considérer les différentes possibilités qui s'ouvrent aux femmes et aux hommes, les moyens à leur disposition et en quoi ces éléments sont modifiés par les activités de développement. L'avantage est évident : la participation des femmes aura une incidence positive sur la réduction de la pauvreté, puisque, on l'a vu, elles jouent un rôle déterminant en la matière. Une attention particulière doit être portée aux divers facteurs qui ont jusque-là empêché les femmes de participer aux négociations internationales et d'accéder à des postes de décision.

Exercer une certaine sélectivité dans le choix des pays partenaires et affecter l'aide là où elle sera le plus efficace peut être fort utile pour renforcer la cohérence des politiques des gouvernements des pays de l'OCDE. Dans ce contexte, la cohérence suppose que les décisions d'affectation des ressources d'APD reposent sur des critères renvoyant avant tout à la réduction de la pauvreté et non à d'autres objectifs dans des domaines comme les échanges, la politique étrangère ou la sécurité nationale.

Le CAD a encouragé ses Membres à soutenir le renforcement de la capacité institutionnelle des pays partenaires de participer aux négociations commerciales internationales, et il a pour cela mis au point un ensemble de bonnes pratiques dans ce domaine. Celles-ci visent à améliorer la coordination de leurs efforts, mais aussi à faire en sorte que le renforcement des capacités soit gouverné par la demande et donne lieu à une appropriation à l'échelon local. Une autre initiative en faveur de la coordination est le Cadre intégré pour l'assistance technique liée au commerce, partenariat qui regroupe six organismes multilatéraux (OMC, Banque mondiale, FMI, PNUD, CNUCED, CCI) afin de coordonner le soutien en faveur de l'intégration du renforcement des capacités et de l'assistance technique en matière d'échanges dans les stratégies nationales de lutte contre la pauvreté des pays les moins avancés.

Il importe en outre de mettre en place des mécanismes qui assurent, dans les négociations internationales, la pleine participation des pays partenaires à l'élaboration et à la conclusion des accords. La Norvège, la Suède et la Suisse proposent une aide au renforcement des capacités dans les domaines de la gestion de la dette, du budget et du contrôle financier. L'Australie, quant à elle, fournit une assistance technique pour les négociations commerciales mondiales.

\section{Domaines d'action}

La suite du chapitre 4 passe en revue un par un les domaines où des réformes s'imposent en priorité pour assurer la cohérence des politiques. La cohérence des politiques suivies par les pays de l'OCDE dans tous les domaines d'intervention des pouvoirs publics est essentielle du fait de l'impact que celles-ci peuvent avoir sur les différentes facettes des relations Nord-Sud. La cohérence des politiques peut influer de multiples façons sur la réduction de la pauvreté mais, pour des raisons pratiques et compte tenu des changements qu'on peut raisonnablement escompter, il a fallu se limiter à n'évoquer dans les présentes Lignes directrices que les domaines les plus importants. La liste des domaines d'action prioritaires proposés, regroupés sous six rubriques thématiques présentée dans l'encadré 16. Ces domaines d'action sont repris succinctement dans le même ordre dans la suite du présent chapitre ${ }^{1}$. 


\section{Encadré 16. Cohérence des politiques : domaines d'action}

- Commerce international et investissement direct étranger Régime commercial international

Régimes commerciaux préférentiels

Échanges de services

Financement des exportations

Investissement direct étranger

Transfert de technologie, notamment TIC

- Mouvements financiers internationaux

Réforme du secteur financier

Investissement de portefeuille

Allégement de la dette

- Alimentation et agriculture

Commerce alimentaire et politique agricole

Aide alimentaire et sécurité alimentaire

Recherche agricole
Ressources naturelles et pérennité de l'environnement

Pollution à l'échelle planétaire

Échanges et investissement

Utilisation des ressources naturelles renouvelables

- Pêche

- Sylviculture

- Questions sociales

Normes du travail

Immigration

Santé publique à l'échelle mondiale

Drogues illicites

- Gestion des affaires publiques et conflits

Démocratie et droits de l'homme

Gouvernement d'entreprise

Prévention et résolution des conflits

Exportations d'armes

\section{Commerce international et investissement direct étranger}

Le commerce international est un instrument puissant de réduction de la pauvreté. Les effets sur le revenu national et le niveau de bien-être des pays en développement que pourrait avoir la suppression ou la réduction des restrictions qui frappent encore les importations en provenance de ces pays seraient gigantesques par rapport à ceux résultant des apports d'APD consentis ces dernières années. Si des réformes visant à libéraliser les échanges ont certes été engagées, d'autres s'imposent et sont envisageables même si elles deviennent plus difficiles à mettre en œuvre politiquement dès lors que des objectifs fondamentaux comme l'emploi et le développement régional sont en jeu. Les principaux domaines de la politique relative aux échanges et à l'investissement où des mesures pourraient être prises pour contribuer à l'amélioration de la cohérence des politiques sont i) le régime commercial international général, ii) les régimes commerciaux préférentiels, iv) le financement des exportations, iv) les échanges de services, v) l'investissement direct étranger et vi) les transferts de technologie. Les subventions agricoles et le commerce alimentaire sont traités plus loin.

i. Le régime commercial international se caractérise par un certain nombre de dispositions qui limitent en particulier l'accès aux marchés des pays de l'OCDE et d'autres pays de produits transformés en provenance des pays en développement. Même si les droits de douane ont été réduits jusqu'à un niveau moyen encore jamais atteint dans le passé, des crêtes tarifaires subsistent sur des produits pour lesquels les pays en développement sont particulièrement compétitifs. La progressivité des droits de douane, en vertu de laquelle les droits augmentent avec le degré de transformation des biens, est une autre forme de restriction qui a pour effet d'entraver les exportations industrielles des pays en développement. Il existe encore en outre des obstacles non tarifaires, comme les normes et réglementations relatives aux produits, les règles d'origine et l'étiquetage des produits, la discrimination par les subventions et mesures compensatoires apparentées, les droits et autres actions antidumping et la non-transparence des procédures de passation de marchés publics, notamment dans le cas des marchés financés par l'aide liée et des achats de navires. 
ii. L'incohérence intrinsèque des différents régimes commerciaux préférentiels, lesquels bénéficient d'ailleurs principalement aux pays à revenu intermédiaire et aux pays en transition, rend d'autant plus difficile la cohérence des politiques commerciales. Or, les avantages en sont limités du fait que ces régimes excluent certains produits sensibles dans certains secteurs où les pays pauvres ont le plus de chances d'intensifier et de diversifier leurs exportations, et peuvent être supprimés unilatéralement si les importations d'un pays augmentent de façon notable. De plus, ces régimes sont souvent trop complexes pour être véritablement compris et appliqués par des administrations déficientes.

iii. Les pratiques et politiques de financement des exportations, y compris les garanties, ont des répercussions sur l'endettement, le développement durable et la réduction de la pauvreté. Les ministres des pays de l'OCDE ont mandaté le Groupe sur les crédits à l'exportation pour qu'il renforce les mesures destinées à assurer que les crédits à l'exportation ne sont pas en contradiction avec les accords internationaux sur le développement durable et, dans le cas des PPTE, ne sont pas utilisés à des fins non productives. En outre, le Groupe sur les crédits à l'exportation a préconisé des mesures destinées à combattre la corruption dans les procédures d'obtention des crédits et à faire en sorte que les crédits soient refusés lorsque les contrats à l'exportation correspondants sont entachés de corruption ${ }^{2}$.

iv. Les échanges de services connaissent actuellement une expansion bien plus rapide que les échanges de biens, du fait en partie des progrès de la technologie, grâce auxquels de nombreux types de services ont enfin pu faire l'objet d'échanges internationaux. L'Accord général sur le commerce des services (AGCS) conclu à l'OMC constitue une avancée importante dans la voie de l'établissement d'un système multilatéral d'échanges de services fondé sur des règles. Même si, dans les faits, il n'a guère contribué à libéraliser les échanges de services, il a permis d'instaurer un cadre pour les négociations futures relatives aux différents secteurs des services. Sur le plan de la cohérence, l'ouverture des marchés des pays de l'OCDE revêt une importance majeure ; les engagements auxquels ces derniers ont souscrit dans le cadre de l'AGCS sont encore loin d'être complets, en particulier dans le domaine des services de main-d'œuvre où des perspectives intéressantes s'ouvrent aux pays en développement.

v. L'investissement direct étranger (IDE) dans les pays en développement a connu une expansion extrêmement rapide ces dernières années, expansion qui a surtout bénéficié aux marchés émergents d'Asie de l'Est et d'Amérique latine ${ }^{3}$. Les pays à faible revenu ne peuvent se prévaloir du cadre stratégique et des conditions institutionnelles, de l'infrastructure, du dynamisme économique et d'un marché de la taille de ceux des nations plus prospères, conditions indispensables pour réussir à attirer l'IDE. Une grande part de ce qu'ils reçoivent est dirigé vers les industries extractives, ce qui n'a qu'un impact limité, voire négatif, sur la stabilité politique et sociale et sur la pauvreté. Les pays en développement doivent être associés en tant que partenaires à part entière à tout processus de négociation en vue d'un accord international sur les règles de l'investissement, lequel pourrait améliorer leur accès à de nouveaux moyens de financement du développement.

vi. Le transfert de technologie vers les pays en développement va généralement de pair avec l'IDE, mais peut aussi s'opérer d'autres manières. L'accord de l'OMC sur les ADPIC ${ }^{4}$ réglemente les transferts de technologie. Il a été conclu en vue de stimuler les dépenses d'investissement dans la recherche-développement de 
nouvelles technologies, non dans l'objectif de réduire la pauvreté. Un certain nombre de questions de cohérence se posent au sujet des brevets relatifs à des biens publics planétaires comme le vivant, la diversité biologique et les médicaments propres à sauver des vies humaines (voir plus loin "questions sociales"). Un autre domaine qui pose problème est celui des technologies de l'information et des communications (TIC), lesquelles pourraient offrir de vastes possibilités aux pays en développement. Il n'en existe pas moins un risque que les pays développés dominent de plus en plus les TIC, ce qui pourrait encore aggraver les inégalités économiques dans le monde. Un effort particulier d'éducation et de formation, et des actions spécifiques visant à faciliter l'accès aux technologies, aux infrastructures, aux conditions du marché, aux cadres juridiques et au savoir, sont donc indispensables.

\section{Mouvements financiers internationaux}

Les années 90 ont vu s'accroître considérablement le niveau des apports internationaux de capitaux aux pays en développement, dans lesquels les capitaux privés l'emportent de loin sur l'APD au total. La déréglementation, l'intégration régionale et les technologies avancées de l'information sont autant de facteurs à l'origine de cette évolution. Cela a certes permis d'acheminer des capitaux fort nécessaires, principalement vers les plus grands et les plus avancés des pays en développement, mais a également entraîné une plus grande instabilité des apports, des problèmes aigus d'endettement et des crises financières plus étendues. Des problèmes de cohérence se posent dans les domaines suivants : i) la réforme du secteur financier, ii) l'investissement de portefeuille et iii) l'allégement de la dette des pays pauvres. Les questions liées à l'investissement direct étranger et au financement des exportations ont été examinées précédemment plus haut.

i. La réforme du secteur financier est importante tant au niveau national qu' international en ce qu'elle renforce l'incitation à veiller à l'efficience de l'investissement et de la croissance économique et qu'elle minimise les risques de crise financière. Un enchaînement ordonné des réformes est déterminant, et celles-ci doivent porter sur la réglementation prudentielle du secteur bancaire, le renforcement des capacités institutionnelles, et l'amélioration de la coordination entre la politique de taux de change, la politique monétaire et les mesures de contrôle des mouvements de capitaux ou leur taxation. Une autre source de préoccupation vient de ce que les pays en développement ont besoin d'être représentés de façon adéquate dans les instances internationales où sont débattues les réformes de l'architecture financière.

ii. L'investissement de portefeuille fournit des ressources financières précieuses, surtout aux pays en développement à revenu intermédiaire dont la cote de crédit est passable ou bonne. Toutefois, les mouvements de capitaux à court terme sont une source majeure d'instabilité, laquelle a elle-même été un facteur important d'aggravation de la pauvreté lors des récentes crises financières dans le monde. Y sont apparentés des problèmes tels que la fuite des capitaux, le secret bancaire et les paradis fiscaux. Dans ce domaine, la cohérence des politiques appelle une reconnaissance de l'ensemble de ces risques et l'adoption de mesures de réglementation prudentielle intégrées dans l'architecture financière internationale et dans les politiques monétaires des pays tant importateurs qu'exportateurs de capitaux.

iii. L'allégement de la dette des PPTE est reconnu à l'échelle internationale comme une condition nécessaire à la réduction de la pauvreté. Pour qu'il donne les effets escomptés, l'allégement de la dette doit venir en sus, et tenir compte non seulement du montant de la dette dont le service aurait pu être assuré, et aurait 
effectivement été assuré, sans allégement, mais aussi du risque qu'il en résulte une diminution des autres formes d'APD et d'autres types de transferts financiers. Les montants sur lesquels doit porter l'allégement doivent être suffisants pour que la dette reste gérable, pour que des investissements puissent être consentis dans la croissance économique et pour que puissent être engagées les dépenses sociales et autres indispensables à un recul de la pauvreté. Les prêteurs qui accordent des crédits aux pays pauvres doivent être conscients des risques et des responsabilités qu'ils prennent et supporter leur part des coûts induits par le non-remboursement de certains prêts.

\section{Alimentation et agriculture}

L'objectif fixé par le Sommet mondial de l'alimentation, qui est de réduire de moitié le nombre de mal-nourris dans le monde d'ici 2015, exige un accroissement de la production alimentaire, en particulier des petits exploitants dans les pays en développement importateurs nets de denrées alimentaires. Cet accroissement revêt une importance majeure pour améliorer la sécurité alimentaire et le revenu des pauvres, hommes et femmes. Les problèmes de cohérence des politiques dans les domaines de l'alimentation et de l'agriculture ont trait i) au commerce alimentaire et aux politiques agricoles, ii) à l'aide alimentaire et à la sécurité alimentaire, et iii) à la recherche agricole et aux biotechnologies.

i. Les politiques relatives aux produits alimentaires, aux produits agricoles et au commerce revêtent une importance cruciale dans la mesure où il s'agit de sujets politiquement sensibles et où elles ont une incidence directe sur les pauvres et les personnes qui vivent dans une situation d'insécurité alimentaire. Une des questions majeures est de savoir quel effet aurait une réduction du soutien des prix intérieurs et des subventions à l'exportation sur les cours mondiaux des denrées alimentaires et, par conséquent, sur le coût des importations des pays en développement importateurs nets de produits alimentaires. La plupart de ces derniers deviennent de plus en plus tributaires des importations alimentaires, et sont de ce fait de plus en plus exposés aux hausses des prix alimentaires : ils risquent donc davantage de subir, indirectement, les effets des politiques agricoles des pays de l'OCDE. Cela dit, une hausse des cours tendrait, à terme, à stimuler leur propre production alimentaire, ce qui les rendrait moins dépendants des importations. Il pourrait en résulter une baisse des prix alimentaires sous l'effet de l'accroissement de la production et des exportations de producteurs à faible coût, mais, en attendant, les pays pauvres en situation de déficit alimentaire risquent d'être confrontés à de graves problèmes de sécurité alimentaire.

ii. Des progrès substantiels ont été accomplis vers une plus grande cohérence des activités de coopération pour le développement dans les domaines de la sécurité alimentaire et de l'aide alimentaire, des formes plus souples d'aide alimentaire prenant peu à peu le pas sur la simple liquidation des stocks excédentaires de produits alimentaires. C'est ainsi que se multiplient les dons destinés à financer des achats sur les marchés locaux ou régionaux, qu'on veille davantage à éviter de perturber ces marchés, que l'éventail des produits offerts est mieux adapté et qu'il est opéré une meilleure prévision des besoins. Il n'en subsiste pas moins des problèmes tels que l'utilisation de l' aide alimentaire pour le soutien des prix agricoles intérieurs, ou le volume insuffisant des produits alimentaires et des ressources financières destinés à assurer la sécurité alimentaire, et l'incohérence des politiques menées par les principaux exportateurs de produits alimentaires.

iii. La recherche agricole revêt une importance fondamentale pour le développement de l'agriculture et la sécurité alimentaire. Les biotechnologies en particulier pourraient largement contribuer à faire refluer la pauvreté si elles étaient 
appliquées dans les pays en développement. Transférer cette technologie aux pays pauvres représente toutefois un défi majeur qui requiert un effort de cohérence des politiques, et ce pour deux raisons essentielles :

- La recherche dans le secteur privé est mue par la demande du marché dans les pays riches plutôt que par les besoins des pays et des populations pauvres. Des mesures spéciales s'imposent donc pour assurer à ces derniers la sécurité alimentaire et l'innocuité des produits alimentaires.

- L'innocuité des produits alimentaires suscite bien des préoccupations, de même que les risques environnementaux, sanitaires et socio-économiques à long terme que peut induire l'utilisation des techniques génétiques. D'où la nécessité d'un étiquetage, de garanties de traçabilité et de mécanismes réglementaires internationaux assurant que les nouvelles technologies sont dûment testées afin de se prémunir contre des risques éventuels ${ }^{5}$. L'adoption de normes internationales et d'accords de reconnaissance mutuelle pourrait permettre d'éviter l'apparition de nouveaux obstacles aux échanges dans ce domaine. Pour donner aux pays en développement une chance de tirer profit des perspectives qui se dessinent et de prévenir les risques liés à l'utilisation des techniques génétiques, il convient de les aider à se doter de solides capacités juridiques, administratives et scientifiques.

\section{Ressources naturelles et pérennité de l'environnement}

Les mesures à prendre pour régler bon nombre des questions se rapportant à la gestion des ressources naturelles et à l'environnement sont avant tout du ressort des pays partenaires. Toutefois, certaines de ces questions ont une portée régionale, voire mondiale, et soulèvent donc des problèmes de cohérence des politiques, notamment dans les domaines suivants : i) la pollution à l'échelle de la planète, ii) l'incidence des échanges et de l'investissement, et iii) l'utilisation des ressources naturelles renouvelables.

i. La pollution à l'échelle de la planète concourt au changement climatique et à la diminution de la couche d'ozone ${ }^{6}$. Les conditions de vie et la vulnérabilité des populations pauvres vivant dans les pays en développement s'en trouveront encore aggravées. Jusqu'à présent, c'est dans les pays de l'OCDE que les émissions sont les plus importantes, mais les pays en développement qui connaissent une croissance rapide les rattrapent petit à petit. La vitesse du changement climatique est particulièrement inquiétante étant donné la capacité limitée des sociétés et des écosystèmes de s'y adapter. La pollution de l'environnement à l'échelle planétaire soulève des questions importantes pour la cohérence des politiques des gouvernements des pays de l'OCDE, tant en ce qui concerne les mesures prises unilatéralement pour lutter contre le réchauffement de la planète et rétablir la couche d'ozone qu'en ce qui concerne les positions adoptées par ceux-ci dans les enceintes internationales compétentes.

ii. Les échanges et l'investissement ne sont pas en soi générateurs de pauvreté ou de dégradation de l'environnement, mais ils peuvent accentuer ou amplifier les distorsions ou les inégalités existantes dans les dotations en ressources. Les règles et les politiques commerciales, le degré d'ouverture des marchés et les caractéristiques des produits modifient les liens complexes existant entre la gestion des ressources naturelles et la réduction de la pauvreté. Les échanges et l'investissement accroissent la valeur des ressources naturelles et incitent à les gérer de façon efficiente et durable. D'un autre côté, lorsque les droits de propriété ne sont pas clairement définis et lorsque les dispositifs régissant l'exploitation des ressources naturelles sont peu ou mal appliqués, la demande accrue de ressources naturelles qu'induit l'ouverture aux échanges peut encore

$\overline{\text { C) OCDE } 2001}$ 
accélérer des modes d'exploitation déjà excessifs. Lorsque l'accès aux ressources naturelles est très inégalitaire, les avantages que procurent les échanges ne font qu'accentuer la disparité des revenus et marginaliser davantage les pauvres.

iii. L'accélération de l'utilisation des ressources naturelles renouvelables dans les pays en développement risque de se révéler intenable et préjudiciable aux intérêts économiques des pays de l'OCDE. La pêche et l'exploitation forestière en sont deux exemples éloquents :

L'investissement direct étranger va souvent de pair avec des transferts de technologies et de procédés de production modernes qui entraînent une utilisation plus efficiente des ressources et une diminution de la pollution et de la production de déchets. Néanmoins, il peut quelquefois se traduire par des transferts de procédés industriels ou d'extraction polluants (dans les secteurs manufacturier, minier et pétrolier) ayant des retombées négatives sur l'environnement, la santé et d'autres aspects sociaux, qui touchent particulièrement les pauvres'. L'exportation de déchets dangereux, qui doit être strictement surveillée pour être compatible avec un développement durable, est un problème apparenté. ${ }^{8}$.

Les ressources de la pêche sont souvent des sources importantes de revenu, de moyens de subsistance et d'emploi dans les pays en développement côtiers, notamment pour les communautés des petits États insulaires. Même si les stocks visés ne sont souvent pas les mêmes, la pêche hauturière des bateaux-usines étrangers peut aboutir à une réduction des prises locales. Dans certains cas, les flottes étrangères bénéficient de subventions et ne supportent donc pas la totalité des coûts de leur pêche, d'où un risque de surexploitation des terrains de pêche. Les industries de transformation du poisson existant dans les pays en développement ou susceptibles de s'y développer, qui peuvent employer des femmes, risquent d'en subir le contrecoup, ce qui détruirait une source importante de réduction de la pauvreté. Le problème de la transformation à grande échelle des mangroves en élevages de crevettes, non viables à long terme, en Asie et en Amérique latine soulève le même genre de problème. La cohérence des politiques dans ce cas supposerait de dresser des bilans et de prendre des mesures pour faire en sorte que les accords bilatéraux sur la pêche à grande échelle dans les eaux des pays en développement, et sur l'élevage des crevettes, contribuent à réduire durablement la pauvreté. Les droits d'accès et les recettes d'exportation pourraient être utilisés pour améliorer la gestion des pêcheries et des zones côtières afin d'instaurer un développement favorable aux pauvres des communautés côtières.

- La déforestation risque d'être perpétuée par l'exploitation des forêts naturelles pour la production de bois d'œuvre, surtout en l'absence de réglementations efficaces concernant le commerce et l'abattage du bois. Dans les pays et les régions les plus pauvres, les mises à blanc opérées par les pauvres qui n'ont pas accès à d'autres sources d'énergie est une autre cause de déforestation. De nombreuses espèces de la faune et de la flore sauvage sont menacées d'extinction par l'exploitation forestière, la chasse et les captures destinées à l'exportation.

\section{Questions sociales}

La réduction de la pauvreté appelle aussi la cohérence des politiques sociales, au plan national comme au plan international. Les problèmes les plus importants dans ce domaine concernent i) les normes du travail, ii) l'immigration, iii) la santé publique à l'échelle planétaire, et iv) les drogues illicites. 
i. Des normes fondamentales du travail internationalement reconnues ont été intégrées dans la version révisée des Principes directeurs de l'OCDE à l'intention des entreprises multinationales, qui s'appliquent aux investisseurs basés dans les pays de l'OCDE et aux filiales de ceux-ci dans le monde entier, y compris les pays en développement. L'OIT a adopté, en coopération avec l'OMC, la CNUCED, la Banque mondiale, le FMI et l'OCDE, un plan d'action pour des conditions de travail décentes, qui relie les droits des travailleurs et le dialogue social aux politiques de l'emploi et de la protection sociale. L'élimination des pires formes de travail des enfants implique, au niveau de la coopération pour le développement, une action en faveur de politiques visant à faire en sorte que les enfants aient totalement accès à l'éducation et que les parents puissent se passer de leur travail et de leurs revenus?.

ii. Tout en encourageant les pays pauvres à ouvrir leurs marchés, les pays de l'OCDE appliquent des politiques de l'immigration restrictives, qui ont pour effet de fermer leurs propres frontières, sauf à l'égard des cadres spécialisés. Comme ceux-ci ont des perspectives de carrière limitées dans les pays en développement, ces politiques d'immigration sélectives contribuent à vider ces pays de leurs ressources humaines. Cet exode des cerveaux pose un important problème de cohérence. Une coopération pour le développement qui viserait à accroître les possibilités d'emploi dans les pays partenaires peut contribuer à résoudre ce problème.

iii. Les problèmes de cohérence intéressant la santé publique à l'échelle planétaire concernent les domaines de la recherche médicale et des prix des médicaments. La recherche biomédicale s'effectue pour l'essentiel dans les pays de l'OCDE et est largement soumise à la loi du marché, c'est-à-dire axée sur les maladies propres à ces derniers dont certaines, il est vrai, constituent également un grave fléau pour les pays en développement. Il n'en reste pas moins que moins de $10 \%$ du total des dépenses publiques et privées allouées à la recherche dans le domaine de la santé sont consacrés aux maladies et problèmes auxquels sont imputables $90 \%$ du fardeau de la maladie dans le monde ${ }^{10}$. Or celles-ci affectent principalement les populations et les pays pauvres, lesquels n'ont pas les moyens d'acheter les médicaments existants qui pourraient sauver ou prolonger la vie, par exemple des victimes du VIH/sida. Cette situation a soulevé un débat sur les droits de propriété intellectuelle, lorsqu'ils sont en conflit avec le règlement de problèmes sociaux majeurs. Bien que l'accord sur les ADIPC conclu dans le cadre de l'OMC prévoie la protection générale de ces droits, il prévoit aussi des dérogations pour la protection de la santé publique. Quelques initiatives importantes ont été prises à cet égard, notamment dans le domaine des incitations à la recherche et du coût des médicaments, mais de nombreuses questions restent en suspens en ce qui concerne la poursuite de l'élaboration des cadres internationaux adaptés.

D'après les estimations de l'OMS, le tabac cause actuellement environ 4 millions de décès par an dans le monde et ce chiffre devrait atteindre 10 millions d'ici 2030, dont $70 \%$ dans les pays en développement. Cela représente un coût humain terrible pour les pays pauvres, en même temps qu'un coup pour leur économie, car la plupart des victimes figurent parmi les travailleurs les plus productifs, sans compter la charge qui en résulte sur leurs systèmes de santé publique. Les gouvernements des pays Membres de l'OCDE pourraient envisager de promouvoir, dans les pays en développement, des mesures visant à décourager l'usage du tabac et à sensibiliser le public aux risques qu'il entraîne pour la santé, comme ils le font chez eux, ainsi qu'à renforcer la responsabilité sociale des sociétés de fabrication du tabac. 
iv. Le problème des drogues illicites est étroitement lié à la pauvreté. Les agriculteurs qui cultivent les plantes d'où l'on tire ces drogues n'ont souvent pas beaucoup de solutions de rechange valables. Les politiques qui ne visent qu'à interdire et à éliminer le trafic de drogue peuvent menacer les moyens d'existence de communautés rurales pauvres. Elles ne seront efficaces à long terme que si les planteurs peuvent trouver d'autres sources de subsistance durables. La drogue (ainsi que des ressources minérales comme le diamant) est aujourd'hui une source majeure de revenus pour les seigneurs de la guerre, les organisations criminelles et les groupes armés engagés dans des guerres internes. Les bénéfices de cette activité illégale sont de plus en plus utilisés pour financer des conflits armés. La lutte contre le commerce de la drogue est donc indissociable des efforts de prévention et de résolution des conflits armés.

\section{Gestion des affaires publiques et conflits}

Il est aujourd'hui généralement admis qu'une réduction durable de la pauvreté ne va pas sans une bonne gouvernance, la stabilité économique et politique et la paix. Les principaux domaines dans lesquels les pouvoirs publics doivent veiller à la cohérence des politiques de ce point de vue sont : i) la démocratie et les droits de l'homme, ii) le gouvernement d'entreprise, iii) la prévention et la résolution des conflits et iv) le commerce des armes.

i. Donner aux pauvres des moyens d'action grâce à l'application des règles de la démocratie participative et des droits de l'homme est indispensable pour faire reculer la pauvreté. La cohérence des politiques est généralement assez bonne au sein des gouvernements des pays de l'OCDE qui font de la lutte contre la pauvreté leur priorité et encouragent la démocratie participative et le respect des droits de l'homme. D'un autre côté, les mesures visant à remédier à une gestion déficiente des affaires publiques, à un manque de transparence et à la corruption dans les pays partenaires peuvent éveiller des susceptibilités politiques ou aller à l'encontre d'intérêts commerciaux ou de politique étrangère, ce qui risque de soulever des problèmes de cohérence.

ii. Les gouvernants sont de plus en plus conscients de l'importance des questions liées au gouvernement d'entreprise et des effets néfastes de la corruption. Il n'en subsiste pas moins d'immenses possibilités d'améliorer la coopération au service d'une meilleure cohérence entre les instances de la zone de l'OCDE qui traitent des questions liées à la grande corruption dans les transactions commerciales internationales, à la petite corruption avec ses effets dommageables pour les pauvres, à la politique de concurrence, aux pratiques fiscales dommageables et au blanchiment de l'argent ${ }^{11}$.

iii. La réduction de la pauvreté est étroitement liée à la prévention et au règlement des conflits. L'absence d'opportunités ou de compétences permettant de trouver un emploi, l'impossibilité de payer les charges scolaires, la pénurie de nourriture et d'eau, et le manque d'accès aux services de santé peuvent inciter les hommes et les jeunes gens pauvres à adhérer à un groupe armé. Des problèmes de cohérence des politiques peuvent se poser si les causes de l'éclatement ou de la persistance de conflits touchent aux intérêts commerciaux, de politique étrangère ou de sécurité nationale des pays de l'OCDE. Faire le maximum pour prévenir et résoudre les conflits, notamment en soutenant les Nations unies et ses forces de maintien de la paix, est un facteur important de cohérence des politiques au service de la lutte contre la pauvreté. 
iv. Les années 90 ont été marquées par une forte augmentation du commerce international des armes - en particulier des petites armes, des armes légères et des mines terrestres - avec des répercussions majeures sur l'accroissement de la pauvreté et du nombre de femmes, d'hommes et d'enfants handicapés. Le chiffre annuel du commerce des armes est estimé à environ 10 milliards de dollars, dont l'essentiel provient d'une douzaine de pays. Le réduire est un défi majeur pour la cohérence des politiques des pays de l'OCDE. 


\section{Notes}

1. Une liste élargie, bien qu'encore incomplète, et uniquement indicative, des domaines d'action dans lesquels il convient de veiller à la cohérence des politiques est adjointe à la Liste des conditions essentielles présentée en annexe, laquelle est conçue comme un document séparé. Parce qu'elle est destinée à un public plus large que les Lignes directrices, elle est structurée différemment et recouvre des aspects de la coopération pour le développement traités dans d'autres chapitres des Lignes directrices.

2. Voir la Déclaration d'action (en date de décembre 2000) du Groupe de l'OCDE sur les crédits à l'exportation à propos de la Convention de l'OCDE sur la lutte contre la corruption d'agents publics étrangers dans les transactions commerciales internationales.

3. L'IDE va à concurrence de $90 \%$ à 23 de ces pays. Sur ces derniers, la Chine et le Brésil ont à eux seuls absorbé la moitié des apports d'IDE aux pays en développement enregistrés en 1998, et dix pays à revenu intermédiaire $70 \%$.

4. Les aspects des droits de propriété intellectuelle qui touchent au commerce.

5. Le Protocole de Cartagena sur la prévention des risques biotechnologiques relatif à la Convention sur la diversité biologique, adopté à Montréal, Canada, en 2000, entrera en vigueur dès qu'il aura été ratifié.

6. En 1996, le Groupe d'étude intergouvernemental sur l'évolution du climat (GIEC) est parvenu à la conclusion que l'observation des faits conduit à penser que les activités humaines exercent une influence indéniable sur le climat de la planète. Les recherches ultérieures ont confirmé cette conclusion. Voir GIEC, Climate Change 2001: The Scientific Basis, Shanghai 2001 (http://www.meto.gov.uk/sec5/CR_div/ipcc/wg1/WGI-SPM.pdf).

7. Foreign Direct Investment and the Environment, OCDE, Paris, 1999.

8. Elle est réglementée par la Convention de Bâle sur le contrôle des mouvements transfrontières de déchets dangereux et de leur élimination (1989). Des problèmes analogues se sont produits avec des pesticides fournis au titre de l'aide, qui se sont révélés représenter un danger pour les utilisateurs et une source de contamination de l'environnement. Voir les Lignes directrices établies à l'intention des organismes d'aide pour la lutte contre les parasites et la gestion des pesticides, Lignes directrices sur l'aide et l'environnement du CAD/OCDE n 6, 1995.

9. Voir La Déclaration et les Décisions sur l'investissement international et les entreprises multinationales. Textes de base, OCDE, Paris, novembre 2000 ; Les échanges internationaux et les normes fondamentales du travail, OCDE, Paris, 2000 ; la Déclaration de l'OIT relative aux principes et droits fondamentaux au travail (1998) et la Convention de l'OIT sur les pires formes de travail des enfants (1999).

10. The 10/90 Report on Health Research 2000, Global Forum on Health Research, OMS, www.globalforumhealth.org.

11. L'OCDE abrite le Groupe d'action financière sur le blanchiment de capitaux (GAFI), dont elle assure le secrétariat bien que ce dernier ne soit pas une instance de l'Organisation. Ce groupe a contribué à améliorer sensiblement la coopération internationale dans la lutte contre le blanchiment de l'argent. 


\section{Liste indicative des conditions essentielles à la cohérence des politiques à l'appui de la réduction de la pauvreté}

Une plus grande cohérence des politiques dans les pays de l'OCDE est en outre indispensable pour que les pays en développement parviennent à tirer pleinement parti des opportunités que leur offre la mondialisation: l'OCDE établira une liste des conditions essentielles à la cohérence des politiques afin d'aider ses pays Membres dans ce domaine. Elle approfondira également ses travaux analytiques sur les liens entre la libéralisation des échanges, la croissance économique et la lutte contre la pauvreté. «Maîtriser la mondialisation», communiqué de presse de la réunion du Conseil de l'OCDE au niveau des ministres, Paris, 27 juin 2000.

\section{Pourquoi centrer les efforts en faveur de la cohérence des politiques sur la lutte contre la pauvreté ?}

Au fil des années, un consensus de plus en plus marqué s'est dégagé des débats à haut niveau de l'OCDE autour de l'idée qu'il faut veiller à la cohérence de l'ensemble des politiques menées par les pouvoirs publics. Les gouvernements ont des priorités multiples, et souvent contradictoires. La réduction de la pauvreté ne l'emporte pas toujours sur les autres priorités dans les décisions que prennent les pays Membres de l'OCDE, même si à l'évidence ceux-ci sont désormais plus disposés à lui accorder une place importante. L'objectif de réduction de la pauvreté suscite depuis quelque temps une adhésion et un soutien politiques très larges à un niveau très élevé tant à l'OCDE qu'à la Banque mondiale, au FMI et à l'ONU et parmi les chefs d'État du G8.

Pourquoi la réduction de la pauvreté mérite-t-elle d'être au centre des efforts d'amélioration de la cohérence des politiques de préférence à quelque autre objectif général ? Pas moins de 1.2 milliard de personnes - soit un cinquième de la population mondiale et un habitant sur quatre des pays en développement - vivent dans une extrême pauvreté, avec moins d'un dollar par jour, et près de 3 milliards de personnes - la moitié de la population mondiale - n'ont pas deux dollars par jour pour vivre. Les responsables politiques des pays de l'OCDE — notamment les chefs d'État du G8 et les ministres des pays de l'OCDE - estiment que la pauvreté est un « mal public planétaire » en raison non seulement de ses conséquences sur le plan économique et humain mais aussi des dysfonctionnements et des désordres qu'elle cause dans les sociétés en développement et, plus généralement, dans le monde. L'instabilité politique, la dégradation de l'environnement, les flux migratoires et autres phénomènes perturbateurs analogues qui en résultent menacent les intérêts des pays Membres de l'OCDE. Ce constat s'est peu à peu imposé. Les objectifs internationaux de développement", y compris celui prévoyant une réduction de moitié pour 2015 de la proportion de personnes vivant dans l'extrême pauvreté, constituent désormais un cadre de référence commun auquel souscrivent le FMI, la Banque mondiale et les Nations unies, de même que les chefs d'État du G8.

Les opposants à la mondialisation, à l'intérieur comme à l'extérieur de la zone de l'OCDE, voient dans les poches de pauvreté qui subsistent dans la prospérité ambiante la manifestation la plus flagrante du déséquilibre des effets de la mondialisation. Nombre de ses partisans, parfaitement conscients que toute modification rapide de la scène économique à l'échelle planétaire fait forcément à la fois des « gagnants » et des «perdants », se rendent eux aussi compte que la mondialisation risque de marginaliser les pays les plus pauvres et aussi des pans entiers de la population des pays en développement à revenu intermédiaire, dès lors

\footnotetext{
*. Voir la liste des objectifs de développement du millénaire, page 17.
} 
qu'une croissance économique asymétrique engendre des inégalités de revenu. Il n'est pas obligatoire qu'il en aille ainsi, mais si cela se produit, la mondialisation et ses multiples avantages s'en trouveront compromis.

La cohérence des politiques est donc indispensable pour que tout un chacun tire profit de la mondialisation. Des politiques cohérentes permettent de combler les asymétries du processus de mondialisation, lesquelles résultent souvent de l'incohérence des politiques aussi bien des pays développés que des pays en développement. Compte tenu des immenses bienfaits qu'elle peut avoir pour la croissance et l'intégration économiques, la mondialisation est un facteur favorable, non un obstacle, à la réduction de la pauvreté. En faisant de la cohérence des politiques à l'appui de la réduction de la pauvreté un objectif primordial, les pays développés renforceront la confiance et le soutien à l'égard de la mondialisation en tant que vecteur d'amélioration du bien-être dans tous les pays du monde.

\section{Pourquoi une liste de conditions essentielles ?}

De très nombreuses décisions des pays développés ont des retombées directes sur la pauvreté dans les pays en développement. Si elles sont cohérentes les unes par rapport aux autres, ces décisions peuvent contribuer à faire reculer la pauvreté. C'est énoncer une évidence que de dire que des politiques cohérentes sont plus efficaces que des politiques incohérentes, mais le défi à relever n'est pas mince. Les questions à traiter font le plus souvent intervenir des groupes d'intérêt nationaux et des services gouvernementaux dont l'objectif premier n'est pas la lutte contre la pauvreté. Il y a parfois conflit entre les efforts qu'appelle la satisfaction des intérêts nationaux et les engagements souscrits à l'égard de la réduction de la pauvreté dans les pays en développement. Par ailleurs, les organismes de développement se trouvent souvent en position de faiblesse par rapport aux autres entités gouvernementales et aux intérêts, publics ou privés, prévalant dans les domaines - échanges, investissement, agriculture et sécurité nationale - dans lesquels un effort de cohérence serait vraisemblablement le plus profitable.

La plupart des gouvernements négligent de promouvoir systématiquement la cohérence des politiques formulées par les divers ministères et services gouvernementaux. Or, le réflexe premier des fonctionnaires et des institutions est de chercher à préserver leur chasse gardée à moins qu'ils ne soient tenus pas des instructions incontournables émanant des centres de pouvoir qui les obligent à veiller à la cohérence des résultats obtenus. Depuis quelques années, les choses commencent toutefois à changer. Dans un éventail de plus en plus large de domaines, les décideurs des pays développés reconnaissent qu'ils ne peuvent donner à leurs gouvernements des conseils avertis sur les problèmes fondamentaux d'orientation de l'action et participer à des discussions et négociations internationales sans tenir compte des conséquences qui en découleraient pour les pays en développement et des réactions de ces derniers, y compris les plus pauvres. L'existence d'un mouvement ONG dynamique, qui rappelle aux pouvoirs publics et à l'opinion les répercussions des politiques suivies sur les pays pauvres, est également importante de ce point de vue. Grâce à ces évolutions, les différentes sphères d'intervention des pouvoirs publics se préoccupent de plus en plus de ce qui peut être fait pour tenir compte de l'impact que les décisions prises peuvent avoir sur la réduction de la pauvreté.

Pour apprécier la cohérence des politiques, il faut disposer de critères et connaître les mécanismes qui lient une politique donnée à la pauvreté internationale. Il importe d'analyser soigneusement le rôle de chaque politique, son impact sur la pauvreté, et dans quels secteurs de l'action gouvernementale des ajustements peuvent être opérés de façon à renforcer la cohérence. Ce genre d'analyse nécessite des moyens appropriés, aussi bien dans les organismes d'aide que dans les autres services gouvernementaux. Ses résultats doivent être communiqués aux décideurs qui participent aux délibérations interministérielles et aux négociations internationales.

Les ajustements qui en découleront pour rehausser la cohérence - dont certains, sinon tous, seront en toute probabilité assez faciles à opérer dès lors qu'on veut s'en donner la peine et que les hiatus de cohérence ont été mis en évidence - seront vraisemblablement le fruit d'un processus politique. Que ces ajustements soient faciles ou non, l'essentiel est toutefois de montrer en quoi ils sont indispensables. Cela demande une analyse solide, des efforts déterminés pour transmettre les connaissances nécessaires aux décideurs et aux parties prenantes et un souci de communiquer des informations fiables et fondées aux médias et à la société civile, instruments majeurs d'information et de sensibilisation de l'opinion publique.

$\overline{\text { C) OCDE } 2001}$ 


\section{Encadré 17. Les différents aspects de la cohérence des politiques}

La cohérence appelle la satisfaction, simultanée qui plus est, d'un certain nombre de conditions, encore qu'il faille bien admettre qu'une totale cohérence ne sera en pratique jamais atteinte.

- La coordination des politiques implique l'instauration d'une collaboration entre les diverses structures institutionnelles et de gestion intervenant dans la formulation des politiques.

- La cohésion des politiques nécessite de faire en sorte que les politiques suivies dans un même domaine n' aient pas des effets opposés et d'éviter toute action qui irait à l'encontre de la réalisation d'un objectif donné, en l'occurrence la réduction de la pauvreté à l'échelle internationale.

- La cohérence des politiques va plus loin. Elle passe par la promotion systématique d'actions se renforçant mutuellement entre les divers domaines d'intervention des différents ministères et instances gouvernementales afin de créer des synergies au service de la réalisation de l'objectif poursuivi.

\section{Mesures à prendre pour assurer la cohérence des politiques}

La cohérence des politiques nécessite :

- Des orientations politiques claires émanant des dirigeants nationaux et une gestion ferme de la part du centre de gouvernement.

L'affirmation claire, par les autorités, des objectifs poursuivis.

- Une réelle consultation et des liens étroits entre les organismes publics, les intérêts privés et la société civile, y compris les ONG. Les organismes de développement entretiennent vraisemblablement de meilleures relations que les autres secteurs de l'administration avec de nombreuses grandes ONG (notamment celles des pays en développement). C'est un atout dont ils peuvent faire bénéficier les ministères des Finances, du Commerce, etc.

- Un renforcement des capacités institutionnelles des services gouvernementaux afin de leur permettre d'analyser ce qu'implique la cohérence des politiques dans certains domaines particuliers et d'y œuvrer dans le cadre de mécanismes efficaces de coopération intragouvernementale.

L'égalité de traitement entre les organismes d'aide et les autres partenaires associés à ces mécanismes institutionnels.

- Une attention accrue de la part des organismes d'aide aux liens avec les organisations multilatérales, avec l'assentiment des instances et services gouvernementaux qui traitent normalement avec ces institutions.

\section{La liste indicative, un outil à l' intention des gouvernements désireux de promouvoir la cohérence des politiques au service de la réduction de la pauvreté}

La liste des conditions essentielles est destinée à encourager, et aider, les gouvernements des pays Membres de l'OCDE à intégrer à leurs processus de prise de décisions des dispositifs et mécanismes de nature à garantir la cohérence des politiques à l'appui de la réduction de la pauvreté. Il ne s'agit que de propositions et d'orientations générales car la conception et le fonctionnement des instruments destinés à assurer la cohérence des politiques risquent de différer très sensiblement d'un pays Membre à l'autre.

La liste se compose de deux parties. La première, axée sur les procédures, énumère les mesures indispensables à l'instauration d'un dispositif destiné à garantir la cohérence des politiques. L'idée maîtresse est en l'espèce la flexibilité. Le contexte dans lequel s'inscrivent l'action des pouvoirs publics et les priorités peut évoluer rapidement et un système efficace doit pouvoir s'adapter à ces évolutions sans qu'il soit nécessaire de le réviser 
en permanence. La seconde partie de la liste recense tout un ensemble d'éléments stratégiques fondamentaux devant faire l'objet d'un examen systématique. Cette partie de la liste doit être regardée comme appelée à être constamment revue et actualisée. Les problèmes mobilisant l'attention des pouvoirs publics sont en effet souvent fonction du moment. Les problèmes de cohérence de demain ne ressembleront pas forcément à ceux d'aujourd'hui, même si la réduction de la pauvreté demeure l'objectif suprême. En outre, cette partie de la liste n'est pas exhaustive et a été volontairement limitée. Chaque gouvernement pourra, et devra, la compléter, et les éléments qui y seront ajoutés différeront vraisemblablement d'un pays à l'autre.

\section{Mesures à envisager pour renforcer la cohérence des politiques à l'appui de la réduction de la pauvreté : liste indicative}

\section{A. Dispositions relatives à l'organisation et aux procédures à suivre}

- Souscrire formellement aux objectifs de développement du millénaire, et

- Afficher clairement et officiellement l'objectif de réduction de la pauvreté et son caractère prioritaire ;

- Mener une campagne d'information de l'opinion publique pour expliquer l'importance de la réduction de la pauvreté à l'échelon international.

- Doter les organismes gouvernementaux des capacités d'analyse leur permettant d'évaluer les liens entre leurs domaines d'intervention et la réduction de la pauvreté et de recenser les points sur lesquels une action s'impose en priorité, et

raccorder ces capacités et priorités aux objectifs de développement du millénaire ;

veiller à intégrer une analyse des sexospécificités dans toutes les études pertinentes.

\section{B. Liste indicative des questions de fond à examiner}

Objectifs des négociations internationales sur les échanges, l'agriculture, les services financiers, l'environnement, les mouvements migratoires, les normes du travail, la gouvernance, les conflits et d'autres domaines connexes, le but étant de rehausser la cohérence à l'appui de la réduction de la pauvreté.

Participation pleine et entière des pays pauvres à toutes les négociations internationales susceptibles d'avoir une incidence sur la réduction de la pauvreté.

Mesures de politique commerciale (par exemple les actions antidumping, l'application de droits compensateurs et les mesures de sauvegarde, les arrangements commerciaux préférentiels, le financement des exportations et les règles de discipline), et compatibilité avec les objectifs de réduction de la pauvreté.

Tarifs douaniers et compatibilité avec les objectifs de réduction de la pauvreté (notamment en ce qui concerne la progressivité des droits de douane et les crêtes tarifaires qui entravent les importations en provenance des pays pauvres).
- Mettre en place un dispositif interministériel ou interorganisations, piloté par un organisme et/ou un groupe de base capable d'obtenir des résultats, pour passer au crible les politiques et les décisions au regard des objectifs de réduction de la pauvreté. Un tel dispositif pourrait comprendre :

des procédures d'échange d'informations entre les différents secteurs gouvernementaux ;

- des systèmes de notification afin que les manquements à la cohérence des politiques observés au sein du gouvernement et sur le terrain soient portés à la connaissance des décideurs et utilisés pour apporter des corrections ;

- des actions de formation et de sensibilisation, dans tous les secteurs de l'administration, sur la réduction de la pauvreté et les ajustements à apporter aux différentes politiques pour y contribuer ;

- des contacts réguliers, au niveau approprié, avec le secteur privé et la société civile, et leur participation.

- Conséquences pour la cohérence des politiques des subventions intérieures ayant des retombées à l'échelon international (par exemple dans les domaines de l'énergie, de l'agriculture et de la pêche).

- Règles d'origine prévues dans la législation et la procédure douanières, en vue en particulier de les adapter à la mondialisation de la production.

- Réglementation nationale relative aux normes, une importance particulière étant attachée à l'accès des importations en provenance des pays et régions pauvres sans déroger aux principes de sécurité des produits et de santé publique, les mesures de précaution devant se fonder sur une évaluation scientifique des risques et non céder à l'influence de pressions protectionnistes.

Politiques financières internationales, en vue de promouvoir une croissance et des investissements favorables aux pauvres tout en minimisant le risque d'instabilité et de crise financière pouvant avoir de graves conséquences pour la pauvreté. 


\section{Mesures à envisager pour renforcer la cohérence des politiques à l'appui de la reduction de la pauvreté : liste indicative}

Accords d'allégement de la dette, lesquels doivent ramener la dette des pays pauvres à un niveau tenable, laisser à ces derniers un volume suffisant de ressources budgétaires pour financer des mesures de lutte contre la pauvreté et opérer un partage des risques adéquat entre les créanciers.

- Initiatives à l'appui du renforcement des capacités des pays en développement, lesquelles doivent être centrées sur les pays les plus pauvres et coordonnées du mieux possible avec les programmes mis en œuvre par d'autres pays et organisations internationales. Accorder une attention particulière à la cohérence des programmes des différents organismes et ministères nationaux et à leur efficience.

- Concours financiers, techniques et sur le plan des politiques destinés à promouvoir l'utilisation des technologies de l'information et des communications (TIC) dans les pays pauvres.

- Budgets nationaux alloués à la recherche médicale et à l'aide, l'objectif devant être d'intensifier les recherches sur les maladies endémiques sévissant dans les pays pauvres, en coopération avec d'autres pays et le secteur privé, selon des modalités qui encouragent la mise en place et le financement de programmes internationaux visant à mettre des médicaments efficaces à la disposition des pauvres.

- Problèmes de cohérence des politiques liés aux ADPIC (par exemple brevets relatifs à des biens publics mondiaux tels que le vivant, la diversité biologique et des médicaments pouvant sauver des vies, connaissances et innovations indigènes, juridictions géographiques).

- Politiques en matière d'aide alimentaire et de lutte contre la faim, en vue d'améliorer la coordination des programmes à l'échelon international, une attention particulière étant portée aux retombées pour la réduction de la pauvreté des ajustements induits par la libéralisation des échanges agricoles.

- Éléments des politiques ayant trait au commerce international des armes susceptibles de favoriser la réduction de la pauvreté et la prévention des conflits, grâce à l'établissement de principes directeurs appropriés.

- Politiques concernant les conflits et la sécurité dans les pays pauvres, afin d'accroître la coordination et d'atténuer le manque de cohésion des mesures prises par les pays développés.

- Mesures en faveur du renforcement des capacités au service d'une gestion efficace, démocratique et transparente des affaires publiques et de la lutte contre la corruption dans les pays à faible revenu.

- Cohérence et adéquation des politiques en matière de gouvernement d'entreprise (par exemple concernant la corruption dans les transactions commerciales internationales, le trafic des stupéfiants, les paradis fiscaux, le blanchiment de capitaux, la réglementation de la concurrence). 


\section{Encadré 18. Cohérence des politiques : liste élargie des domaines d'action}

1. Échanges internationaux de biens et de services ; investissement direct étranger (IDE) ; politiques connexes

Questions relatives aux négociations internationales

Droits de douane

Obstacles commerciaux non tarifaires

Normes applicables aux produits et services

Réglementation des échanges de biens et de services

Marchés publics, aide liée

Règles d'origine

Subventions et droits compensateurs

Arrangements commerciaux préférentiels

Régimes antidumping

Droits de propriété intellectuelle (DPI)

Accords internationaux sur l'investissement

Crédits et garanties de crédit publics à l'exportation (y compris crédits mixtes et crédits agricoles)

Renforcement des capacités

Gouvernement d'entreprise

Politique de la concurrence

Optimisation des effets de l'IDE dans les pays pauvres

Paradis fiscaux et concurrence fiscale dommageable

Questions relatives au transfert de technologie

Production et commerce de médicaments génériques et leur mise à la disposition des pauvres (en partie liés aux DPI)

TIC : questions relatives au fossé numérique

\section{Alimentation et agriculture}

Politiques agricoles

Commerce agricole

Sécurité alimentaire

Aide alimentaire

Lutte contre la faim

Recherche agricole et biotechnologies

Organismes génétiquement modifiés (OGM) et commerce de leurs produits

\section{Ressources naturelles et viabilité environnementale}

Environnement mondial (par exemple changement climatique, couche d'ozone, diversité biologique)

Environnement régional et infrarégional (pluies acides, pollution marine, etc.)

Environnement local : préservation et problèmes de santé publique (par exemple pollution de l'air, des eaux et des sols)

Exploitation durable des ressources renouvelables (par exemple pêche, forêts)

Utilisation des ressources non renouvelables et atténuation des effets dommageables qui en résultent pour l'environnement et au plan social

Impact des échanges et de l'investissement sur l'environnement

\section{Questions relatives à la gouvernance}

Démocratie et droits de l'homme

Transparence

Qualités d'écoute des institutions publiques

Lutte contre la corruption

Organisation et professionnalisation de la fonction publique

Droits relatifs au travail

\section{Conflits et sécurité}

Prévention et résolution des conflits

Commerce des armes

\section{Questions sociales}

Enseignement et formation

Filets de protection sociale

Systèmes de santé publique

Migrations

Questions de santé publique telles que les maladies tropicales ou le tabagisme

\section{Questions financières et économiques générales}

Politique macro-économique

Politiques de surveillance structurelle

Architecture financière internationale

Blanchiment de capitaux 


\section{Mettre le changement et le développement institutionnels au service de la réduction de la pauvreté, du partenariat et de la cohérence des politiques}

\section{Introduction}

Pour lutter efficacement contre la pauvreté, il faut intégrer le principe de la réduction de la pauvreté à toutes les politiques et activités des organismes d'aide, se comporter en bons partenaires et rehausser la cohérence des politiques au sein des gouvernements des pays Membres du CAD ${ }^{1}$. Ces défis, les organismes de développement ne pourront les relever avec succès qu'en procédant à de profonds changements de leurs institutions, c'est-à-dire de leurs modes d'organisation, de leurs mécanismes d'incitation, de leurs pratiques, de leurs systèmes et de leurs cultures. La rénovation de la capacité institutionnelle des organismes d'aide et l'adaptation de leurs systèmes de gestion à ces défis rendront d'autant plus efficaces leurs efforts de mise en œuvre des présentes Lignes directrices, et leur contribution à la réalisation des objectifs de développement.

Ce chapitre est centré sur la structure organisationnelle des organismes d'aide et contient diverses suggestions susceptibles de contribuer à mieux accorder les dispositifs institutionnels aux objectifs de réduction de la pauvreté, de partenariat et de cohérence des politiques ${ }^{2}$. Modifier la structure institutionnelle d'un système d'administration publique est une tâche complexe qui exige souvent de l'imagination, des innovations et une impulsion vigoureuse de la hiérarchie. Le CAD et ses Membres continueront d'étudier les moyens qui s'offrent de promouvoir le changement institutionnel à mesure que grandira leur expérience en la matière et que se multiplieront les exemples de bonnes pratiques.

\section{Pourquoi remettre en cause nos institutions ?}

En 1998, le Réseau informel du CAD sur la réduction de la pauvreté (POVNET) a fait réaliser une évaluation des résultats obtenus dans le domaine de la lutte contre la pauvreté par chaque organisme d'aide bilatérale. Les conclusions de cette étude approfondie (DAC Scoping Study of Donor Poverty Reduction Policies and Practices, OCDE, Paris, 1999) montrent que les performances des organismes d'aide, appréciées à la mesure de leur détermination à faire reculer la pauvreté ainsi que du ciblage et du suivi des activités menées à cet effet, ne sont par à la hauteur des objectifs affichés.

L'étude a mis en évidence trois problèmes auxquels se sont heurtés les organismes d'aide à des degrés divers :

- L'absence de message clair de la hiérarchie témoignant de sa détermination à combattre la pauvreté, d'où une superposition d'objectifs concurrents, et des défaillances dans le ciblage des mécanismes de gestion et de contrôle ainsi que des programmes par pays.

Les performances passées des organismes d'aide n'ont pas été à la hauteur de leurs objectifs affichés et ce souvent parce que les composantes de leur organisation n'étaient pas totalement adaptées à l'objectif de lutte contre la pauvreté. 
Les organismes de développement qui atteignent les objectifs qu'ils se sont fixés sont ceux qui sont parvenus à établir une cohérence et une bonne imbrication de toutes les composantes de leur organisation stratégie, travail, personnel, culture et dispositifs organisationnels formels et informels.
Des règles institutionnelles, des pratiques de gestion et des systèmes d'incitation qui tendent à renforcer les fonctions de contrôle des organismes d'aide, pérenniser la pression à l'utilisation des crédits, empêcher l'établissement de synergies avec d'autres partenaires au développement, décourager l'adoption d'une démarche plus intégrée et plus transversale et perpétuer la mainmise des donneurs sur la programmation.

- Des systèmes d'évaluation et de gestion des performances axés sur les apports de ressources et les résultats mesurables, comportant peu de références à l'obligation, pour les organismes d'aide ou le personnel, de rendre compte de leurs réalisations au regard des objectifs de développement en général et des objectifs assignés à chaque organisme.

Ces problèmes sont caractéristiques d'institutions dont les structures, les systèmes et les cultures ne sont pas encore bien adaptés aux objectifs qu' elles se sont fixés. Pour mener à bien l'action de lutte contre la pauvreté convenue par les Membres du CAD et énoncée dans les présentes Lignes directrices, il faudra apporter d'importants changements, qui pourront varier selon le contexte ${ }^{3}$. Les organismes d'aide poursuivent bien entendu aussi d'autres objectifs mais de tels changements sont d'autant plus importants que la pauvreté est considérée comme une cible fondamentale ou centrale.

\section{Définitions et concepts}

Un organisme d'aide est avant tout une organisation. Pour déterminer comment les organismes d'aide peuvent se transformer de l'intérieur, il est essentiel de comprendre ce qu' est une organisation, comment elle fonctionne et comment elle peut atteindre une efficacité maximale.

Qu' entend-on par organisation? Les organisations sont des systèmes sociaux complexes composés de quatre éléments entretenant des relations étroites :

- Les personnes : direction, encadrement et personnel.

- Le travail : activités accomplies par les agents.

- La structure formelle : structures, processus et systèmes permettant d'organiser les activités et de guider les personnes dans l'accomplissement de leur travail.

La structure informelle (ou culture interne) : valeurs, positions et convictions défendues par l'organisation, canaux de communication et courants d'influence non officiels, et normes de comportement acceptées.

Les organisations ont aussi une conception de leur mission et une stratégie qui leur permettent d'orienter leurs travaux dans une direction particulière et d'atteindre certains objectifs.

Une organisation qui réussit est une organisation qui travaille dans le sens de « l'adéquation organisationnelle », de la « congruence » ou de la « convergence », c'est-à-dire qui a établi une cohérence et une bonne imbrication de toutes ses composantes internes - stratégie, travail, dispositifs organisationnels formels et informels, et personnel. Il n' existe pas de structure exemplaire, ni de culture exemplaire, ni de stratégie exemplaire unique : l'important est l'interaction de ces composantes. Trop souvent, les organisations imitent certaines de leurs homologues (en particulier lorsqu'elles utilisent les mêmes réseaux et partagent les mêmes objectifs), adoptant ainsi des pratiques qui ne correspondent pas forcément à leur stratégie ou à leur structure organisationnelle. Il en résulte des dysfonctionnements et des résultats décevants au regard des objectifs fixés et des efforts déployés. 
A titre d'exemple, les caractéristiques d'un organisme d'aide dont toutes les composantes tendent de manière cohérente vers la réduction de la pauvreté sont les suivantes :

- La hiérarchie est acquise à la cause de la lutte contre la pauvreté et en témoigne de façon systématique et visible, en paroles et en actes, qu'elle s'adresse au personnel, aux pays partenaires ou au grand public.

- La structure organisationnelle facilite les interactions du personnel, les échanges de compétences et les flux de communication nécessaires pour combattre la pauvreté sous tous ses aspects.

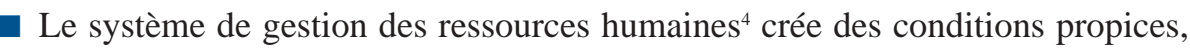
sur le plan de l'organisation et de la gestion, notamment grâce à des dispositifs appropriés d'incitation, à la mobilisation des connaissances, des énergies et de la volonté requises pour placer la pauvreté au centre de l'action engagée.

- Les systèmes et pratiques internes - tels que règlement et statuts du personnel, contrats, procédures d'approbation et de vérification - renvoient expressément à l'objectif de réduction de la pauvreté de l'organisation et renforcent les obligations de comptes à cet égard.

- Le personnel s'applique à intégrer les considérations de lutte contre la pauvreté dans ses activités et à présenter cette dernière comme un objectif primordial de la politique gouvernementale dans ses contacts avec des représentants d'autres secteurs de l'administration dans les capitales, sur le terrain et dans les instances internationales.

- L'objectif de réduction de la pauvreté est une constante du système d'aide dans son ensemble, y compris les politiques et activités des organismes exécutants et des consultants, les positions adoptées au sein des instances dirigeantes des institutions multilatérales de développement et les activités d'aide au développement menées par d'autres secteurs de l'administration.

Qu' entend-on par changement organisationnel ? Les organisations changent pour améliorer leurs chances de succès. Le résultat des changements organisationnels apportés est conditionné par les éléments suivants :

- L'existence d'un projet précis concernant la situation ou le devenir souhaité, ralliant l'adhésion de tous, afin de susciter une volonté de changement.

- Une impulsion forte de la hiérarchie, qui orchestre elle-même le processus de changement.

Une large participation.

- Une bonne communication (horizontale et verticale), permettant notamment un partage d'information sur les résultats obtenus.

- L'adaptation des systèmes et procédures de l'organisation.

- Le recours au travail d'équipe.

- La conscience de l'importance de la culture, laquelle peut faciliter ou ralentir le changement.

Certains organismes de développement ont entrepris de modifier leurs structures ${ }^{5}$, souvent pour mieux tenir compte de l'orientation nouvelle de leurs activités au service de la lutte contre la pauvreté. C'est ainsi que le PNUD, le DFID au Royaume-Uni, l'Asdi en Suède et la Banque mondiale ont entrepris de modifier leurs structures formelles et
Un certain nombre de Membres du CAD ont entrepris de modifier leurs structures, souvent pour mieux tenir compte de l'orientation nouvelle de leurs activités au service de la lutte contre la pauvreté. 
Figure 3. Mesures permettant d'intégrer progressivement la réduction de la pauvreté et la décentralisation dans un organisme de coopération pour le développement

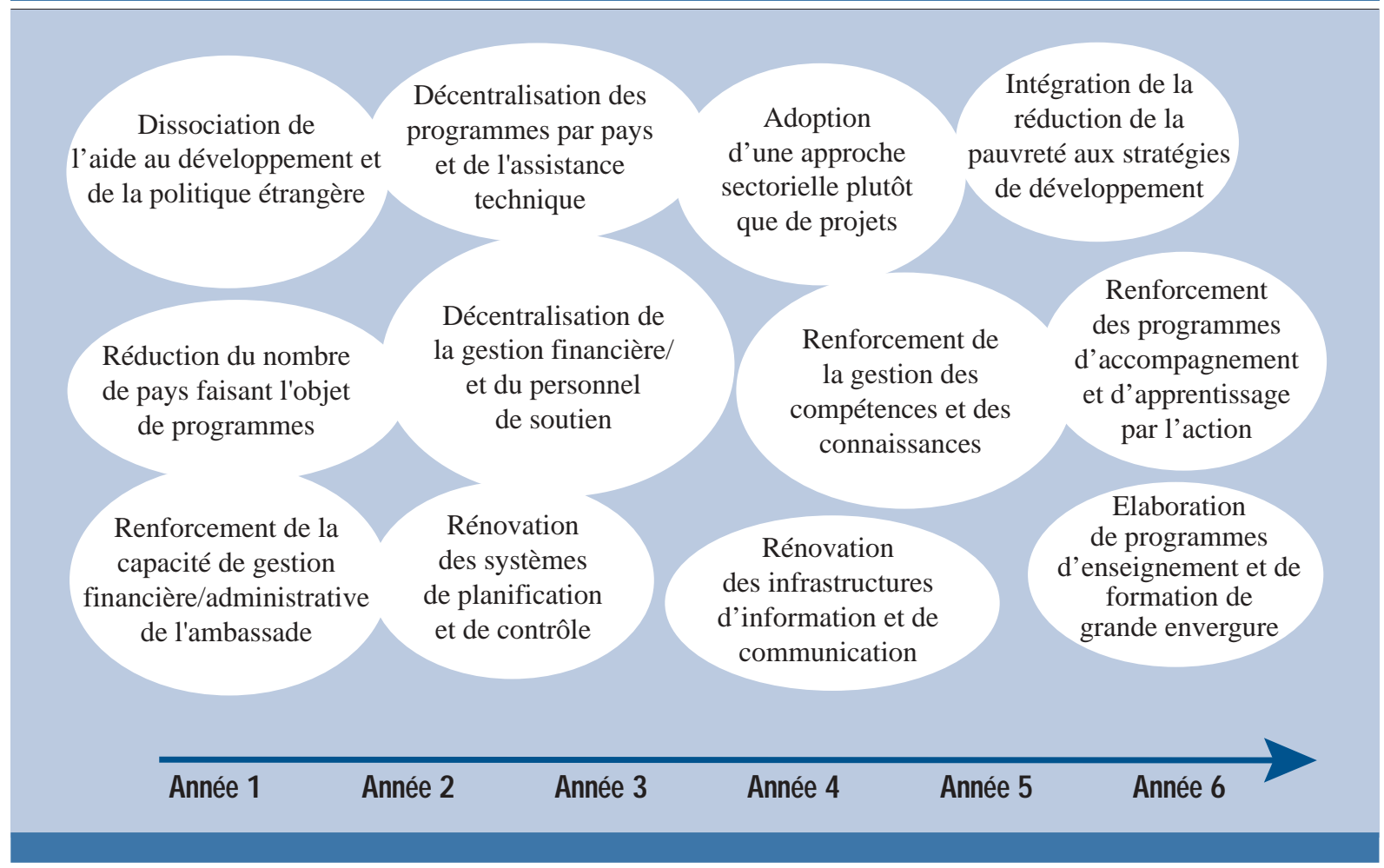

Le changement institutionnel nécessite non seulement qu' on adopte des perspectives, des fonctions et des attitudes nouvelles mais aussi qu'on " désapprenne " certains comportements. informelles, de même que leurs méthodes de travail. De leur côté, l'ACDI au Canada, le Danida au Danemark, le BMZ en Allemagne et l'USAID revoient leurs systèmes de suivi et de gestion des performances. Tous ces changements ont pour but de trouver la bonne formule pour accorder les structures, systèmes et méthodes formels des ces organismes à leur volonté accrue d'œuvrer à la réduction de la pauvreté, au partenariat et à la cohérence des politiques. La figure 3 montre les mesures que pourrait prendre tout organisme pour commencer à adapter les différentes « pièces » de son organisation aux objectifs stratégiques de réduction de la pauvreté et de partenariat.

Il n'existe pas de modèle unique qui permette de faciliter le changement institutionnel - processus long et itératif. Celui-ci exige en effet d'adopter des perspectives, des fonctions et des attitudes nouvelles mais aussi de « désapprendre » certains comportements. Chaque organisme doit formuler son propre diagnostic afin d'évaluer comment, dans quelle mesure et à quel rythme il peut - et va - procéder aux changements nécessaires pour mettre en œuvre et atteindre ses objectifs de réduction de la pauvreté et de partenariat.

Le processus de changement sera en outre affecté par le cadre administratif et l'environnement politique dans lesquels s'inscrivent les activités de chaque organisme. Les organismes de coopération pour le développement ne sont qu'une composante de systèmes complexes d'administration publique, dont les règles et procédures peuvent limiter les options qui s'offrent à eux pour procéder à des changements institutionnels. Parallèlement, ils subissent l'influence d'une multitude d'acteurs, qui peuvent varier d'un Membre du CAD à l'autre, parmi lesquels les parlements et autres instances législatives, les hommes politiques, les autres secteurs de l'administration, les organismes exécutants, la société civile, les ONG, le monde des affaires, les consultants et les milieux universitaires. Les possibilités de changement institutionnel différeront d'un 
organisme à l'autre en fonction du degré de liberté qu'autorisent le système d'administration publique, la dynamique politique interne et les pressions exercées par l'opinion publique. Faire de la lutte contre la pauvreté une constante est une tâche ardue qui nécessite un projet, du temps, un bon ordonnancement et la capacité d'opérer des choix dans un cadre institutionnel complexe. Beaucoup peut cependant être fait pour adapter la culture, les structures et les pratiques des organismes d'aide à l'objectif de réduction de la pauvreté.

\section{Tirer parti au mieux des divers modèles d'organisation}

Les organismes bilatéraux ont des structures et des fonctions différentes. Les organismes Membres du CAD peuvent être répartis en cinq grandes catégories :

- Les organismes intégrés au ministère des Affaires étrangères, au sein duquel les questions de coopération pour le développement relèvent, avec celles relatives à la politique étrangère et aux relations commerciales, de bureaux géographiques (par pays ou par région) intégrés.

- Les directions ou divisions chargées de la coopération pour le développement au sein du ministère des Affaires étrangères.

ש Les organismes assujettis à un ministère de tutelle et associés à un (des) organisme(s) d'exécution distinct(s).

- Les pays où la coopération pour le développement relève de divers ministères, dont chacun s'occupe d'un aspect particulier du programme et qui font appel à des organismes d'exécution distincts.

- Les organismes autonomes.

Les organismes sont très différents par leur taille, leurs attributions, leur champ d'activité, les compétences et l'âge de leur personnel, leur degré de décentralisation et le degré de primauté de l'objectif de réduction de la pauvreté. Ils diffèrent aussi par leurs méthodes de travail, leurs cultures organisationnelles, leur degré d'autonomie, leurs avantages comparatifs et leur organisation fonctionnelle.

Cependant, ils ont aussi de nombreux points communs, en particulier lorsqu'ils se fondent sur des modèles fonctionnels similaires ou qu'ils partagent des affinités culturelles. La figure 4 compare les différents organismes de développement en fonction de leur structure et, en regroupant les organismes similaires, donne une idée des possibilités qui pourraient s'offrir de resserrer la collaboration afin d'abaisser les coûts de transaction. La collaboration entre organismes est vraisemblablement d'autant plus facile qu'ils utilisent la même langue, sont proches sur le plan culturel et partagent des approches similaires de la gestion et du contrôle financier, que leur degré de décentralisation est comparable et que leurs dirigeants entretiennent des relations professionnelles étroites.

L'établissement de partenariats déborde le simple cadre du changement organisationnel dans les organismes d'aide. Il suppose des relations différentes et des échanges entres les organismes ainsi qu'une plus grande collaboration, en particulier sur le terrain. Mieux informés sur la manière de travailler des autres organismes, les Membres du CAD pourront déterminer comment établir une collaboration et comment faire ensemble œuvre plus efficace en exploitant les synergies et les complémentarités.
Chaque organisme

Membre du CAD est un cas unique qui se différencie des autres par ses méthodes de travail, son organisation fonctionnelle, sa culture organisationnelle et les objectifs qu'il poursuit.

II existe cependant aussi de nombreux points communs entre les organismes d'aide. Mieux informés sur ces similitudes et sur la façon de travailler des autres organismes, les Membres du CAD pourront déterminer comment établir une collaboration et faire ensemble œuvre plus utile. 
Figure 4. Les cinq structures de base des organismes de coopération pour le développement des Membres du CAD

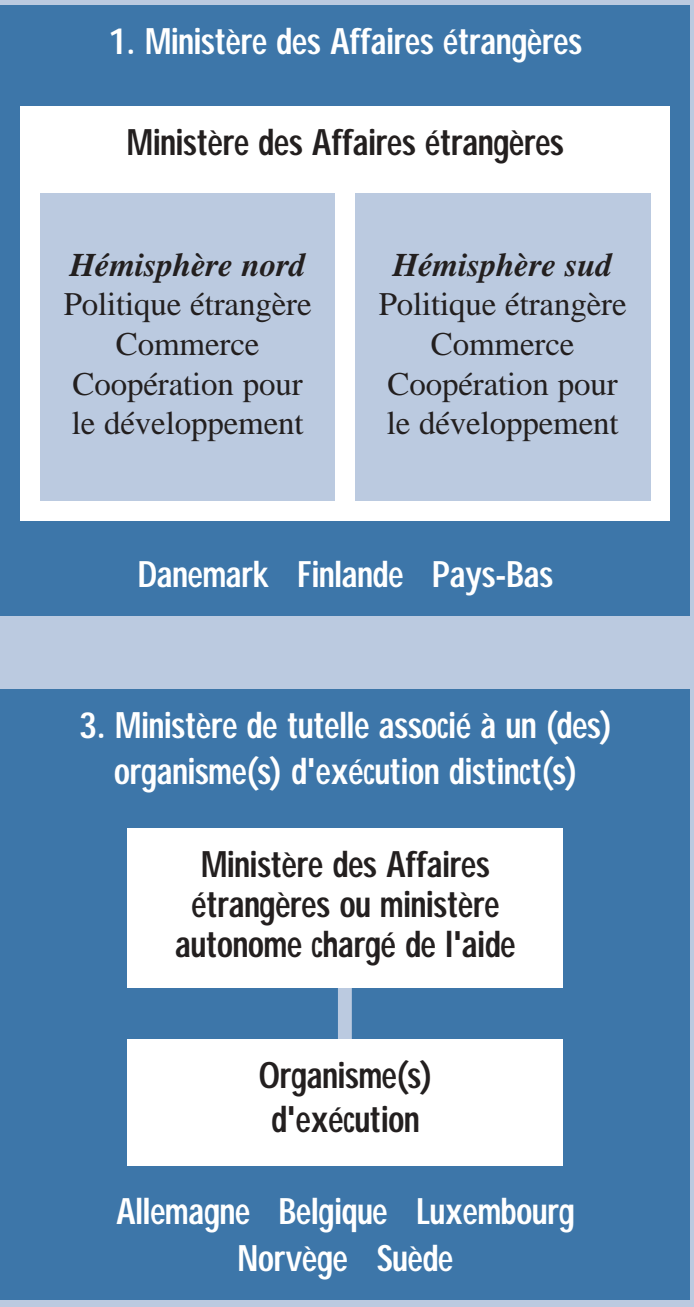

5. Organisme d'aide ou ministère autonome

Australie Canada États-Unis Royaume-Uni
2. Direction ou division de la coopération pour le développement au sein du ministère des Affaires étrangères

\section{Ministère des Affaires étrangères}
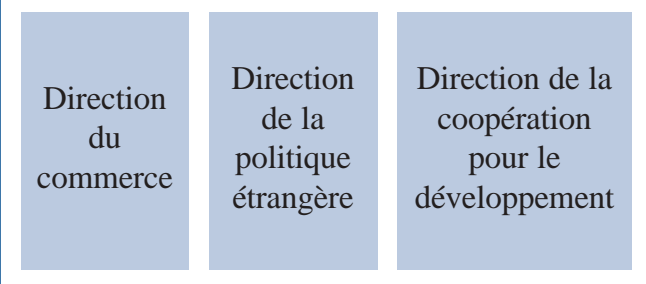

Irlande Italie Nouvelle-Zélande Suisse

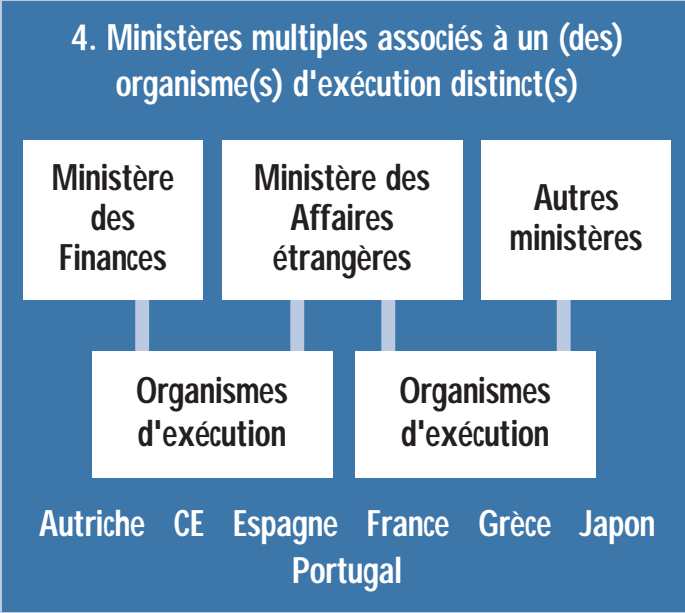

Des structures similaires peuvent faciliter la coordination des activités des organismes d'aide et la collaboration sur le terrain (recherche, mise en ouvre) ou au niveau des services centraux (formation, réseaux d'information). La collaboration est aussi plus facile lorsque les organismes utilisent la même langue, sont proches sur le plan culturel et partagent des approches similaires de la gestion et du contrôle financier, et/ou que leur degré de décentralisation est similaire. 


\section{Faire en sorte que tous les services de l'organisme d'aide tiennent compte de l'objectif de réduction de la pauvreté}

Promouvoir la réduction de la pauvreté au moyen des stratégies et d'actions d'un organisme d'aide nécessite beaucoup plus que la création d'un service spécialisé chargé de la lutte contre la pauvreté. Cela implique de veiller à ce que tous les services adoptent de façon systématique une ligne d'action axée sur la réduction de la pauvreté, ce qui se traduira par une prise en compte des objectifs de réduction de la pauvreté dans toutes les activités menées et à tous les niveaux hiérarchiques.

Il n'existe pas qu'une seule voie pour y parvenir : une méthode efficace dans un organisme pourra ne pas l'être dans un autre. Les éléments du modèle organisationnel qui interviennent dans la prise en compte des objectifs de réduction de la pauvreté sont essentiellement : les responsables de l'organisme, les systèmes et les pratiques, les compétences, les structures et les politiques de gestion des ressources humaines, en particulier la gestion des performances, les incitations et la formation. Une description en est donnée ci-dessous, assortie de conseils et de solutions possibles pour la mise en œuvre des changements requis. En étudiant ces différentes solutions, il est essentiel de garder à l'esprit l'importance d'une bonne concordance organisationnelle, garantissant que les différentes pièces du puzzle de l'organisation s'assemblent parfaitement (par exemple que le système de gestion des performances et les incitations sont compatibles avec l'objectif de réduction de la pauvreté).

\section{Le rôle des responsables de l'organisme d'aide : se donner une ambition, s'engager et créer une culture propice au changement}

La détermination des dirigeants et de la hiérarchie à combattre la pauvreté est décisive pour la création d'une culture propice à l'adhésion à cet objectif de l'organisme dans son ensemble. Le rôle joué par les responsables dans la définition des objectifs, des valeurs et de la mission de l'institution préfigure le sens de l'action à venir. L'exemple offert par la hiérarchie est très important : c'est en effet auprès de ses dirigeants que le personnel cherche instructions, inspiration et conseils. Par des messages clairs (sous forme de déclarations, discours et notes d'information) diffusés dans toute l'organisation, et un comportement allant dans le même sens, les dirigeants peuvent contribuer à susciter un climat de détermination à l'égard de la lutte contre la pauvreté, à aligner les actes sur la parole.

Les responsables des organismes d'aide jouent aussi un rôle essentiel pour instaurer une culture propice au changement à l'intérieur de leurs organisations. Il est difficile de déclencher des changements dans les cadres institutionnels. Le personnel doit faire face à des problèmes, à des pressions, à des tiers, il a peu de temps pour réfléchir à d'éventuels changements, ou n'en voit pas l'intérêt. Le changement n'est pas perçu comme positif : il est perturbateur, dérangeant et menaçant. Lorsqu'on voulait procéder à des changements par le passé, on les imposait souvent d'autorité, mais ce n'était pas toujours très efficace. Les changements décidés par la direction sans véritable consultation du personnel suscitent l'hostilité, la suspicion et la méfiance, qui se traduisent à leur tour par une opposition à la fois ouverte et latente. Les changements qui se produisent alors dans l'organisation ne s'accompagnent pas de changements dans les comportements : il s'agit de changements superficiels ne suscitant pas une véritable adhésion.

Le personnel doit être conscient de la nécessité du changement, le souhaiter et être associé à la planification des mesures et à l'élaboration des méthodes nécessaires. Les organisations qui ont réussi à obtenir un véritable changement d'état d'esprit ont adopté une démarche ouverte offrant au personnel de fréquentes possibilités d'exercer une
« Intégrer » les considérations de lutte contre la pauvreté signifie prendre en compte les objectifs de réduction de la pauvreté dans toutes les activités et à tous les niveaux hiérarchiques de l'organisme d'aide.

Les responsables des organismes d'aide jouent un rôle essentiel pour instaurer une culture de nature à rallier I'adhésion de l'ensemble des services à l'objectif de réduction de la pauvreté ainsi qu'à favoriser et gérer le changement. 
Les systèmes et procédures institutionnels notamment les procédures d'examen préalable et d'approbation des projets, le règlement du personnel et les mandats des consultants et travaux de recherche - doivent établir un lien direct avec les objectifs de lutte contre la pauvreté. influence sur le processus, de façon à susciter une adhésion forte de sa part et à l'inciter à faire sienne la dynamique du changement. Les organismes d'aide doivent être conscients qu'ils ne peuvent espérer d'évolution que s'ils réussissent à instaurer une culture institutionnelle propice au changement, ce qui ne sera possible que si des efforts exprès sont déployés pour promouvoir et gérer le changement.

Mesures à envisager

Une action déterminée doit être engagée dans les plus hautes sphères, tant au niveau politique qu'au niveau de l'élaboration de l'action gouvernementale, afin de mobiliser et de focaliser l'intérêt et la volonté de l'ensemble du personnel, des autres instances publiques et de la société civile, et d'œuvrer plus résolument et plus fermement en faveur de la réduction de la pauvreté dans les pays partenaires.

L'organisme d'aide doit se doter d'un projet précis, d'un cadre d'action et d'une stratégie de lutte contre la pauvreté.

La fixation d'objectifs multiples (développement durable, réduction de la pauvreté, égalité homme-femme, conflits ou objectifs nationaux de politique étrangère, par exemple) est créatrice de tensions. Les dirigeants doivent clarifier ces objectifs en consultant largement le personnel, afin de déterminer les points de complémentarité, de faciliter les arbitrages et d'éviter des différends lors des débats ultérieurs.

Il est essentiel de mettre les responsables des ressources humaines en relation avec le personnel chargé de la formulation des politiques : les dirigeants doivent comprendre les implications concrètes que les stratégies et politiques retenues ont au niveau de la gestion du personnel et du renouvellement des ressources humaines.

Les responsables de l'organisme, à tous les niveaux - et particulièrement au niveau des cadres intermédiaires - doivent proclamer haut et fort leur engagement au service de la lutte contre la pauvreté.

Rénover les systèmes et les processus institutionnels

Les organismes d'aide disposent de nombreux systèmes qui leur permettent d'organiser, de contrôler, de faciliter leur travail et de gérer leur personnel. Les objectifs de réduction de la pauvreté doivent être pris en compte dans ces systèmes afin d'en affiner le ciblage et de renforcer la discipline.

Mesures à envisager

L'objectif de réduction de la pauvreté doit imprégner l'ensemble des processus de planification de l'organisme d'aide (qu'ils se rapportent aux stratégies par pays, aux approches sectorielles ou aux projets). Les programmes et projets doivent faire l'objet d'une évaluation systématique axée sur leur contribution potentielle à la réduction de la pauvreté dans le cadre du système de sélection et des procédures d'approbation en vigueur dans l'organisme.

- Des révisions du règlement du personnel ou des ajouts à ce règlement peuvent être envisagés afin de renforcer la position de la réduction de la pauvreté en tant qu'axe central des objectifs, des activités et des résultats de l'organisme d'aide.

- Il convient de promouvoir une culture institutionnelle favorable à la lutte contre la pauvreté. Un état d'esprit privilégiant l'utilisation à tout prix des crédits 
ou ne témoignant que d'un intérêt de façade pour la réduction de la pauvreté peut diluer ou compromettre le ciblage des activités de l'organisme d'aide sur la pauvreté. Il faut donc trouver des méthodes, des instruments ou des stratégies qui permettent d'empêcher ou d'atténuer les pressions qui s'exercent en fin d'exercice budgétaire pour décaisser les crédits restants.

Les cahiers des charges relatifs aux projets de recherche, aux études et à l'élaboration des programmes doivent faire expressément référence aux objectifs de réduction de la pauvreté. Des objectifs et des étalons en matière de réduction de la pauvreté doivent figurer dans les contrats et les accords de partenariat conclus avec des organismes extérieurs, des ONG et des consultants. Ces documents doivent prévoir des dispositifs incitatifs et des sanctions, ainsi que des systèmes clairement définis de suivi des performances.

\section{Nouvelles capacités et compétences requises}

Il a été constaté dans le cadre de l'étude approfondie (Scoping Study) du CAD que les organismes de développement n'avaient pas nécessairement cherché à déterminer si la nature et le niveau des compétences de leur personnel correspondaient à leurs objectifs prioritaires de réduction de la pauvreté et à leurs nouvelles méthodes de travail. Or, les compétences requises dépendent de la manière dont un organisme est structuré et des méthodes qu'il considère comme optimales pour une réduction effective de la pauvreté.

Une évaluation des compétences dans tous les services de l'organisme permettra de répertorier les connaissances à acquérir ou à approfondir, en ayant recours à la formation, au recrutement de personnel nouveau, aux services de consultants, à des échanges ou à des détachements de personnel d'autres organismes. Il faut pouvoir compter sur un large éventail de compétences spécialisées (y compris les connaissances nécessaires pour traiter des questions transversales telles que l'égalité homme-femme, l'environnement, la gestion des affaires publiques et les approches participatives). Pour la mise en œuvre des stratégies de réduction de la pauvreté, il conviendra de faire davantage appel à des équipes pluridisciplinaires (d'où la nécessité de savoir constituer des équipes). Il faudra aussi améliorer la capacité du personnel d'interpréter des informations quantitatives et qualitatives sur la pauvreté et de se servir de connaissances interdisciplinaires.

Il faut non seulement faciliter l'acquisition de compétences, mais aussi prendre des mesures réfléchies pour regrouper les qualifications. Il s'agit de renforcer la capacité du personnel de raccorder des informations macro-économiques et sectorielles, de combiner des connaissances généralistes et spécialisées en favorisant l'émulation par le travail d'équipe, et d'apporter sur le terrain des conseils éclairés aux points critiques du processus de planification et de mise en œuvre.

\section{Mesures à envisager}

Exploiter l'ensemble des compétences disponibles au sein de l'organisme d'aide et aider le personnel chargé des différents secteurs à acquérir des compétences dans le domaine de la réduction de la pauvreté et à les intégrer à son travail.

S'efforcer de constituer de «nouvelles compétences» :

- Pour le partenariat : capacité de médiation et de coordination et aptitude à établir des relations (écoute active, aptitude à faire naître un consensus, sens de la négociation, diplomatie).
Le traitement des problèmes liés à la pauvreté nécessitera le recours à un éventail plus large de compétences spécialisées (dans des domaines tels que le développement participatif, le renforcement des capacités statistiques et la gestion des affaires publiques) ainsi qu'un effort de regroupement des compétences sur le terrain par la constitution d'équipes pluridisciplinaires.

L'édification de partenariats sur le terrain requiert des compétences nouvelles, notamment des capacités de médiation et de coordination, de la diplomatie, des dons d'écoute active, l'aptitude à faire naître un consensus et le sens de la négociation. 
Une structure organique permettant de traiter efficacement les multiples dimensions de la pauvreté est une structure qui favorise l'échange de connaissances, l'émulation et l'amélioration de la coordination.
- Pour le personnel diplomatique : aptitude à comprendre les problèmes de développement, à prendre des risques et à collaborer avec les partenaires sur le terrain.

- Pour le personnel de terrain : aptitude à faire preuve de souplesse, capacités d'adaptation et d'autocritique, aptitude à élargir le champ de sa réflexion.

Pour tenir compte de toutes les dimensions de la pauvreté, il faut constituer et déployer, à l'échelon de chaque pays, des équipes pluridisciplinaires possédant des compétences dans de nombreux domaines. Réunir des compétences d'ordre macro, méso et micro-économiques.

Instituer des procédures de recrutement (pour le personnel permanent, temporaire et diplomatique) privilégiant les compétences et les performances dans le domaine de la lutte contre la pauvreté, la capacité de travailler en équipe et l'expérience de la coordination (aptitude à faciliter le dialogue, sens de l'écoute et de la négociation).

Encourager la mise en place de «guides» du personnel, capables de relier les compétences et connaissances de terrain à l'analyse générale au siège -et de confronter ainsi la théorie et la pratique.

\section{Structures}

La pauvreté est pluridimensionnelle et nécessite des actions dans des domaines divers comme la santé, l'éducation, les infrastructures, le développement des microentreprises et le renforcement de l'autonomie. Il faut adopter des stratégies globales et intégrées qui tiennent compte de ces différentes facettes à l'échelle des divers secteurs, régions et groupes sociaux. Il s'agit d'une approche très différente des modalités « traditionnelles » de l'aide se fondant sur une succession unidimensionnelle et relativement simple d'interventions au moyen de projets. Cette évolution exigera des organismes de développement qu'ils acquièrent une plus grande connaissance des pays partenaires (y compris du point de vue des pauvres eux-mêmes) et une bonne compréhension des problèmes sectoriels, des relations intersectorielles et des bonnes pratiques.

La mise en œuvre d'une stratégie pluridimensionnelle efficace de lutte contre la pauvreté peut être considérablement facilitée par une structure organisationnelle qui favorise l'échange de connaissances et de compétences spécialisées, l'émulation et l'amélioration de la coordination. Les structures en vigueur, qui répartissent généralement les membres du personnel dans des unités autonomes et isolées s'occupant du développement de secteurs ou de zones géographiques particulières, font obstacle aux échanges, à l'établissement de synergies et à la cohérence des stratégies.

Sur le plan structurel, les organismes de développement ont eu recours à quatre approches différentes, inspirées en grande partie des bonnes pratiques mises en évidence pour l'intégration de la problématique homme-femme, pour améliorer leur capacité institutionnelle d'assurer la prise en compte à tous les niveaux des questions de pauvreté :

Création d'unités spécialisées chargées d'intégrer la réduction de la pauvreté aux activités d'aide au développement.

Désignation de « relais » ou de « chefs de file » chargés de faciliter la mise en œuvre de mesures et le changement institutionnel (travaillant souvent en étroite collaboration avec l'unité spécialisée).

Association de « chefs de file » de la lutte contre la pauvreté et d'unités ayant autorité sur l'ensemble du personnel pour promouvoir la lutte contre la pauvreté. 
Mise au point d'une structure matricielle « allégée » combinant la réduction de la pauvreté (structure par programmes) avec une organisation géographique/régionale (structure fonctionnelle).

On peut aussi recourir avec profit à d'autres dispositifs structurels plus souples afin de mobiliser des connaissances et des compétences spécialisées au service de la lutte contre la pauvreté. La constitution d'équipes pluridisciplinaires (chargées d'accomplir des tâches spécifiques) et de réseaux (permettant de diffuser l'information et les enseignements tirés de l'expérience dans tous les services de l'organisme d'aide) peut contribuer sensiblement à l'amélioration de l'impact de l'aide en termes de réduction de la pauvreté. Ces formules auront d'autres conséquences sur les modes d'organisation des effectifs et de collaboration entre agents, ainsi qu'au niveau de l'accès aux technologies et à la formation.

Il n'existe pas d'approche structurelle qui puisse à elle seule convenir à toutes les situations, car les modalités et les contextes de mise en œuvre varient beaucoup. Sans compter qu'une structure est plus que la somme des composantes d'un simple organigramme. Elle comprend aussi les interfaces ou les «blancs $»^{6}$ les cases de l'organigramme. Il est inévitable que les structures des organismes d'aide diffèrent, chacune devant répondre à des contraintes et à des besoins différents sur le plan de l'organisation.

\section{Mesures à envisager}

Encourager le travail en équipe dans un souci de décloisonnement entre les spécialités, de façon à permettre de mieux appréhender le caractère pluridimensionnel de la pauvreté et de dépasser le stade des approches strictement sectorielles ou des stratégies conduites par les donneurs.

- Mettre en place des structures et des mécanismes pour intégrer de façon systématique la lutte contre la pauvreté dans les activités d'aide au développement. Il est possible de désigner des «chefs de file » de la réduction de la pauvreté, chargés d'accroître l'importance accordée à la lutte contre pauvreté dans l'organisme d'aide, de dispenser des conseils, d'améliorer la communication horizontale et verticale entre les niveaux hiérarchiques et de promouvoir les bonnes pratiques. Des ressources et des pouvoirs doivent être délégués aux personnes qui, dans l'organisme, se font les apôtres de la lutte contre la pauvreté.

- Des structures moins hiérarchisées et plus simples sont plus propices au développement du travail d'équipe, à l'enrichissement et à la valorisation de compétences pluridisciplinaires parmi le personnel, et à un élargissement de la place faite aux flux et réseaux d'information. D'un autre côté, une certaine hiérarchisation est indispensable pour assurer la reddition de comptes, le contrôle de la qualité et la cohésion des décisions.

- Il est essentiel de prendre conscience que, en dépit de l'importance fondamentale des structures organisationnelles, ce sont les méthodes de travail informelles dont dépendent les modalités du travail en groupes et de la collaboration d'une structure à l'autre - qui comptent le plus.
La constitution

d'équipes pluridisciplinaires pour accomplir le travail de terrain et l'établissement de "réseaux de connaissances » pour assurer un partage de l'information et des enseignements tirés de l'ex périence entre tous les services de l'organisme d' aide sont des dispositifs organisationnels qui peuvent se révéler extrêmement utiles.

Aussi importants que soient les dispositifs organisationnels formels (" relais ", réseaux ou unités chargés de promouvoir la lutte contre la pauvreté), ce sont les méthodes de travail informelles - dont dépendent les modalités du travail en groupes et de la collaboration entre les différents services - qui comptent le plus. 
La gestion des performances du personnel, les systèmes de récompense et $d$ 'incitation et les actions de formation - éléments qui influent tous considérablement sur la motivation et le zèle des agents doivent être conçus de manière à appuyer les objectifs de lutte contre la pauvreté et de partenariat.

\section{Assurer la compatibilité des pratiques de gestion des ressources humaines avec l'objectif de réduction de la pauvreté}

La gestion des ressources humaines joue un rôle essentiel dans la sélection, le renforcement et le renouvellement de l'atout le plus précieux d'un organisme de développement : son personnel. La gestion des performances, les systèmes de récompense et d'incitation, ainsi que la formation, influent considérablement sur la motivation et le zèle du personnel, et, en tant qu'éléments centraux des politiques de gestion des ressources humaines, doivent être mis en conformité avec les objectifs de réduction de la pauvreté de l'organisme d'aide.

\section{Gestion des performances et incitations}

Toutes les organisations ont recours à des systèmes de gestion, de suivi et d'évaluation des performances du personnel et à des dispositifs d'incitation. Ce sont à la fois les méthodes de gestion et ces mécanismes primordiaux qui garantissent l'accomplissement du travail. En l'absence de définition, de mise au point et de gestion de tels mécanismes et systèmes, il n'existe aucun cadre dans lequel inscrire les performances du personnel et du système, ni aucun élément moteur. Dans une telle situation, l'organisme, même animé des meilleures intentions, travaille à vide, et les activités mises en œuvre manquent souvent leur cible.

Souvent, les objectifs et les stratégies de l'organisation ne sont pas reliés aux mécanismes tels que les systèmes de gestion des performances ou d'incitation. L'étude approfondie du CAD (DAC Scoping Study) a montré que les incitations et les récompenses existant dans les organismes d'aide n'étaient pas rattachées à l'objectif de réduction de la pauvreté. De ce fait, l'attention portée à la lutte contre la pauvreté varie considérablement d'un service à l'autre, d'un programme à l'autre et d'une personne à l'autre. Dans ces conditions, les mécanismes de suivi et d'évaluation ou les systèmes d'incitation sont en décalage avec l'attachement affiché aux objectifs de réduction de la pauvreté.

Tenir effectivement compte dans l'évaluation des performance du zèle que met chaque agent à œuvrer à la réduction de la pauvreté, se comporter en bon partenaire et promouvoir la cohérence des politiques nécessitera la mise au point de critères et de jalons appropriés. Un suivi périodique, par la hiérarchie, des systèmes d'évaluation du personnel et de l'organisme dans son ensemble peut se révéler extrêmement utile pour apporter des éléments d'information sur lesquels fonder son jugement. La performance en matière de réduction de la pauvreté doit dans toute la mesure du possible être appréciée au regard de l'impact obtenu. Cela ne va pas sans problème car il est parfois difficile d'opérer dans les faits une relation de cause à effet avec telle ou telle activité. Il faudra élaborer des méthodes pour l'évaluation des progrès et des résultats obtenus en matière de soutien des efforts de lutte contre la pauvreté déployés à l'échelon local, y compris des indicateurs supplétifs pour les cas où il est impossible d'établir un lien de causalité.

L'établissement d'indicateurs de performance du personnel à partir des objectifs de l'organisme (comme la réduction de la pauvreté) ou de ses valeurs institutionnelles (comme l'aptitude à constituer des équipes ou à travailler en partenariat) est une tâche difficile. Celle-ci peut être facilitée si les objectifs et les critères de performance correspondants sont directement liés, à différents niveaux hiérarchiques (directions, sections, divisions, unités, etc.) à des objectifs et à des valeurs institutionnels d'importance primordiale. Un tel système de gestion des performances « en cascade » accentue et amplifie les efforts réalisés dans toute l'organisation pour répondre aux objectifs de réduction de la pauvreté et favoriser des comportements et des réflexes de partenariat.

Les récompenses et les incitations contribuent à façonner et à renforcer le comportement du personnel et jouent un rôle essentiel pour stimuler les performances. 
Les récompenses non monétaires peuvent prendre différentes formes : élargissement du choix des missions ou des postes à l'étranger, attributions plus ambitieuses et plus intéressantes, visibilité plus grande, temps libre pour d'autres activités professionnelles, accès à des formations spéciales dans l'optique d'un élargissement des possibilités de carrière, financement de travaux de recherche ou d'études spécialisées, promotion, etc.

\section{Mesures à envisager}

- Il convient de renforcer les liens entre les objectifs stratégiques de l'organisme, les programmes d'activité des unités et les «objectifs de résultats »assignés aux membres du personnel, afin d'accrô̂tre la cohérence interne et de consolider les efforts déployés.

- Des objectifs précis en matière de réduction de la pauvreté et de partenariat doivent figurer dans les mandats et les évaluations des performances des directeurs chargés des différents pays et des gestionnaires de programme, ainsi que dans les critères d'évaluation de leur personnel.

- Pour être crédibles, les systèmes de gestion des performances doivent se fonder sur des critères objectifs. Dans la mesure où la réduction de la pauvreté fait partie des critères utilisés, les organismes d'aide peuvent être appelés à centrer leurs efforts sur la mise au point de méthodes d'évaluation des effets des activités mises en ouvre sur la réduction de la pauvreté.

Les systèmes d'incitation doivent être souples, faciles à mettre en ouvre et prévus dans les textes.

- Il est important de répertorier et de comprendre les facteurs institutionnels qui poussent les gens à agir ou les en dissuadent (explicitement et implicitement) pour déterminer les mesures susceptibles d'accrôttre la cohérence entre les objectifs de réduction de la pauvreté de l'organisme et les performances du personnel.

\section{Apprentissage et formation}

Pour inciter une administration à adopter un nouveau mode de fonctionnement, il faut prévoir d'importants efforts d'apprentissage et de formation concernant les nouvelles activités et le nouvel environnement institutionnel. Le personnel devra être sensibilisé aux questions et aux préoccupations que suscite la pauvreté. Il lui faudra apprendre à « s'identifier » aux objectifs, aux valeurs et aux engagements nouveaux de l'organisation. De nouvelles compétences devront être acquises. La formation peut aussi jouer un rôle important dans l'amélioration de l'efficacité de l'aide par la transmission des bonnes pratiques et des enseignements tirés de l'expérience.

Il faut adapter la formation dans le domaine de la lutte contre la pauvreté au public visé en prévoyant des cours différents :

Des cours d'initiation destinés aux généralistes, aux agents nouvellement recrutés et au personnel de terrain (en particulier au personnel recruté localement) permettront d'apporter des rudiments essentiels sur la pauvreté : ses aspects, son ampleur, les principaux moyens de s'y attaquer, etc. De tels cours contribuent à mieux faire connaître la mission et les valeurs de l'organisme au regard de l'objectif de réduction de la pauvreté.

- Des cours de mise à niveau destinés aux agents débutants, au personnel désireux d'élargir ses connaissances en matière de développement et au personnel de terrain (y compris au personnel recruté localement) permettront d'aider ces derniers à se familiariser avec des sujets tels que les objectifs de développement
Transformer le mode de fonctionnement d'une administration nécessite de sensibiliser le personnel aux questions liées à la pauvreté, de l'aider à « $s$ 'identifier 》 aux valeurs et objectifs nouveaux de l'organisation et de renforcer la base de connaissances de cette dernière. 
Se faire mutuellement profiter de leurs processus d'apprentissage et de leurs actions de formation et faire de même avec leurs partenaires, échanger des informations sur leurs méthodes et matériels de formation sont autant de moyens pour les organismes d'aide d'améliorer la qualité et le rapport coût-efficacité de leurs actions de formation. du millénaire, les nouvelles notions et approches de la pauvreté, les raisons qui conduisent à privilégier l'aide-programme sur les projets, la conception et la mise en œuvre du soutien à apporter, etc. Ce type de formation améliore les compétences du personnel de terrain qui peut ainsi mener un travail d'équipe plus efficace.

Les experts techniques ont pour leur part besoin de cours ciblés et plus approfondis leur permettant d'enrichir leurs connaissances et leurs qualifications et de se tenir au courant des recherches et des expériences les plus récentes.

Des formations doivent aussi être prévues à l'intention des consultants, des agents d'autres services de l'administration et de la société civile afin de leur donner un aperçu des notions, des besoins et des défis en relation avec la pauvreté, et de les aider comprendre en quoi leur travail peut influer sur la réduction de la pauvreté.

\section{Mesures à envisager}

Les actions de formation doivent favoriser une sensibilisation de l'ensemble du personnel à la lutte contre la pauvreté et améliorer les compétences opérationnelles des personnels spécialisés.

Les organismes d'aide peuvent se faire profiter mutuellement de leurs processus d'apprentissage et de leurs actions de formation, et en faire profiter les partenaires. La collaboration pour la mise au point de cours de formation particuliers est extrêmement utile à l'échelon du pays, où elle peut être inscrite dans le cadre des travaux d'élaboration des stratégies locales de lutte contre la pauvreté. La planification et la formation doivent aller de pair.

Les organismes de développement doivent échanger des informations sur leurs instruments, leurs méthodes et leurs matériels de formation et les mettre à la disposition du public (par exemple sur leur site web), de façon que d'autres organes puissent y faire leur choix et adapter ces matériels à leurs propres besoins.

Les cours de formation à l'intention des formateurs intervenant dans le domaine de la réduction de la pauvreté revêtent une grande importance et pourraient être mis au point et dispensés en collaboration avec d'autres organismes.

Les méthodes de formation par la pratique et les programmes d'apprentissage par l'action axés sur la résolution de problèmes réels rencontrés sur le terrain se sont révélés extrêmement efficaces.

- Il faut donner au personnel du temps pour acquérir et diffuser les bonnes pratiques appliquées dans le domaine du développement, et lui faciliter l'accès aux connaissances, aux travaux de recherche et aux résultats des exercices d'évaluation.

\section{Travailler en partenariat}

Le partenariat amène certains Membres du CAD à déplacer vers l'échelon local le centre de gravité de l'élaboration des politiques et de la prise de décision. Il accentue aussi la nécessité de réduire le fardeau que font peser sur les pays partenaires les procédures et les contrôles imposés par les organismes d'aide. Ces éléments ont des répercussions directes sur les changements à apporter aux systèmes, structures et capacités des organismes de développement. 


\section{Décentralisation}

L'importance accrue accordée au partenariat et à l'appropriation locale incite les organismes d'aide à décentraliser la prise de décision et le personnel vers leurs instances sur le terrain. Cette décentralisation les aide à mieux appréhender les conditions locales de la pauvreté, à mieux réagir en fonction de leur évolution. Elle favorise en outre le dialogue et le partenariat, grâce aux contacts étroits et permanents qu'elle permet avec d'autres partenaires locaux, et elle renforce la crédibilité de l'organisme en tant que partenaire. L'expérience montre que la décentralisation a certes un coût élevé, mais aussi de grands avantages.

La décentralisation a des répercussions notamment sur les mécanismes de prise de décision et de communication. Elle suppose une plus grande souplesse budgétaire et une délégation de pouvoirs et de compétences en matière de négociation avec les partenaires locaux. Les organismes qui ont entrepris ou achevé de décentraliser leurs activités déclarent généralement s'être heurtés à des problèmes au niveau du déploiement du personnel, de la circulation des informations et des mécanismes de reddition des comptes.

La décentralisation se traduit par une évolution des tâches et des responsabilités des gestionnaires et du personnel des bureaux locaux, du rôle des services centraux et des modes de collaboration entre les services centraux et les missions locales, évolution qui nécessite de repenser l'organisation existante des activités, y compris les dispositifs organisationnels formels et informels.

- Le personnel de terrain - y compris le personnel diplomatique - a besoin de compétences spécialisées et de connaissances adaptées pour prendre des décisions éclairées concernant les programmes et pour établir des relations efficaces avec ses homologues locaux, ce qui a des conséquences au niveau du recrutement, de la formation, de l'affectation des experts ou des équipes et des compétences à mettre à la dispositions des bureaux locaux pour leur permettre de gérer les transactions financières.

- Les services centraux devront renoncer à leur mainmise sur les transactions et les relations avec les partenaires pour se consacrer davantage à la gestion stratégique globale, en veillant à la logistique de telle sorte que le personnel de terrain reçoive en temps voulu les informations et les ressources dont il a besoin, en définissant de grandes orientations et des normes, en établissant un dispositif léger d'assurance de la qualité, en facilitant l'accès aux moyens et réseaux de communication, en gérant les systèmes de passation des marchés et en se faisant les apôtres de la cohérence des politiques entre les différents ministères et services gouvernementaux. Les bureaux géographiques devront être renforcés pour que leur personnel puisse faire face aux nombreuses tâches nouvelles que lui impose la décentralisation.

La décentralisation nécessite des efforts redoublés de coordination du dialogue et des négociations à l'échelon local afin d'éviter de multiplier les réunions et d'exercer des pressions excessives sur les capacités des pouvoirs publics. Elle exige aussi une gestion prudente du personnel recruté localement - de façon à éviter les surcharges de travail, à ne pas accaparer toutes les compétences locales, et à offrir des possibilités d'évolution de carrière. Elle demande de l'imagination pour trouver des moyens de faire partager la culture organisationnelle des services centraux et leur manière de voir à l'ensemble du personnel des missions locales. En outre, elle rend plus indispensable encore une amélioration des mécanismes d'apprentissage permanent aux différents niveaux de l'organisme, de façon à assurer la diffusion des connaissances et à garantir que

\author{
La décentralisation \\ de la prise de \\ décision et du \\ personnel vers les \\ instances de terrain \\ contribue largement \\ à favoriser un \\ resserrement des \\ relations de \\ partenariat et à \\ promouvoir \\ l'appropriation locale \\ des choix \\ stratégiques et des \\ activités mises en \\ œuvre.
}

Dans un organisme
décentralisé, la
mission des services
centraux n'est plus
de gérer les
transactions et les
relations mais de se
concentrer sur la
gestion stratégique
d'ensemble, de
faciliter l'accès aux
moyens et réseaux
de communication et
de promouvoir la
cohérence des
politiques à l'échelle
de l'ensemble de
l'administration.

$\overline{\text { (C) OCDE } 2001}$ 


\section{Encadré 19. Quelques conseils pour une décentralisation réussie}

Les principaux éléments d'une décentralisation réussie sont les suivants :

- Un cadre général clair et un « centre » solide ayant des idées précises sur la manière de décentraliser le contrôle et les activités.

- Des dirigeants capables d'assurer une bonne gestion des personnes et des processus au siège et sur le terrain.

- Une souplesse qui permette de modifier les fonctions et les tâches du personnel des services centraux.

- Des bureaux locaux dotés d'un personnel possédant l'éventail de compétences voulu (compréhension générale des questions de développement, efficacité personnelle, compétences spécialisées et bonne connaissance du pays partenaire et des méthodes de travail des services centraux).
- Des communications de bonne qualité (intranet, systèmes efficients d'information pour la gestion, téléconférences, réseaux thématiques virtuels).

- Des réunions physiques régulières du personnel en poste dans les pays, dans les antennes régionales et au siège.

- La désignation, au siège, de « correspondants » des agents de terrain, de façon à faciliter les échanges d'information et l'accès aux services offerts par le siège, et à renforcer le sentiment d'appartenance et la solidarité.

- Le personnel d'ambassade doit être formé et habilité à prendre des décisions éclairées, à gérer le processus de changement et à créer des pouvoirs jouant le rôle de contrepoids.

l'expérience acquise est exploitée dans toute la structure de gestion décentralisée. L'encadré 19 recense quelques bonnes pratiques pour la décentralisation de la prise des décisions et de la gestion au profit des bureaux locaux.

Avant de décider de décentraliser, il faut mettre en balance les avantages à attendre d'un tel processus et les facteurs négatifs potentiels, comme l'accroissement des coûts et les pressions excessives qui risquent d'en résulter sur les compétences techniques de l'organisation. En outre, il n'est pas toujours utile ni possible de procéder à une décentralisation pour des organismes d'envergure modeste. L'amélioration de la collaboration et le partage des compétences et des informations entre tous les organismes, ainsi que le recours accru aux compétences locales, sont donc d'autant plus importants.

\section{Mesures à envisager}

Le processus de décentralisation et le rythme auquel il se déroulera dépendront de l'impulsion venue d'en haut, des structures, de la culture interne et du passé de l'organisation, ainsi que de la situation prévalant dans le pays partenaire.

Les besoins d'information s'accentuent en cas de décentralisation (la constitution de réseaux et la communication prennent davantage d'importance, les courants d'information réciproque deviennent essentiels, le personnel doit entretenir des contacts et mettre le siège au courant de ces contacts).

- Il est important d'aborder de front les difficultés inhérentes à la décentralisation en termes de gestion des ressources humaines (critères de sélection du personnel de terrain, incitations financières dont il convient d'assortir les affectations sur le terrain, évolution de carrière du personnel local, conditions de réintégration dans les services centraux, etc.)

La mondialisation a incité de nombreuses entreprises privées à décentraliser leurs activités : la réalisation d'évaluations et d'études de cas, afin de tirer les enseignements de leur expérience, pourrait utilement guider les efforts de décentralisation des organismes d'aide. 
Alléger le fardeau que font peser les organismes de développement sur leurs partenaires

Les exigences administratives multiples des organismes d'aide et la mauvaise coordination de leurs politiques et de leurs activités font peser une lourde charge sur les gouvernements partenaires, en particulier dans les pays où sont présents de nombreux organismes. Le consensus qui se dégage en faveur de la prise en main par les pays des stratégies de lutte contre la pauvreté accentue encore la nécessité de simplifier et d'harmoniser les pratiques, les procédures et les obligations en matière de notification dans le respect des exigences de transparence de chaque organisme.

Souvent, il faut dans le même temps appuyer les efforts de renforcement des capacités du pays partenaire (par exemple dans les domaines de la gestion financière, de la reddition de comptes, du suivi, etc.) afin d'assurer la transparence à l'égard des parties prenantes. Ce renforcement des capacités ne doit pas se limiter au secteur public mais être étendu au secteur privé et à la société civile (associations professionnelles, par exemple). Le renforcement des capacités administratives et de leur probité accroîtra la disposition des organismes de développement à aligner leurs systèmes et procédures sur ceux des pays partenaires. Les Membres devraient envisager, en collaboration avec d'autres Membres, de revoir leurs exigences administratives et d'assouplir leurs procédures financières. De telles évolutions sont essentielles pour faire face aux défis de l'appropriation locale des stratégies de lutte contre la pauvreté et du partenariat.

\section{Mesures à envisager}

Les organismes d'aide doivent contribuer au renforcement de la capacité des pays partenaires de gérer l'aide au développement et de rendre des comptes sur son utilisation, notamment en établissant des rapports et en contrôlant les activités menées. A mesure que les capacités se renforceront et que la confiance des organismes d'aide à l'égard des dispositifs locaux de reddition des comptes s'accroîtra, une évolution progressive vers des mécanismes de contrôle plus souples pourra être envisagée (acceptation des normes appliquées par les partenaires en matière de comptes rendus et/ou de rapports).

- Il faudrait étudier les possibilités de resserrement de la collaboration entre les organismes d'aide en vue de coordonner les missions et les activités de suivi et d'évaluation dans un même pays.

Les organismes d'aide pourront être appelés à aider les partenaires à se mettre mieux à même de conduire les efforts de coordination.
La simplification et l'harmonisation des procédures de gestion de l'aide et des obligations, dans le respect des exigences de transparence de chaque organisme, sont des conditions sine qua non du partenariat. 


\section{Une des grandes priorités des organismes d' aide doit être de s'assurer que les politiques suivies dans d'autres domaines d'intervention des pouvoirs publics - par exemple les échanges, l'agriculture, l'environnement, les migrations ou l'allégement de la dette - ne risquent pas de saper leurs efforts de réduction de la pauvreté.}

\section{Améliorer la capacité institutionnelle de favoriser la cohérence des politiques}

Le succès de la lutte contre la pauvreté nécessite une amélioration de la cohérence des politiques à l'échelle de l'ensemble des gouvernements Membres de l'OCDE, et pas uniquement dans le domaine de la coopération pour le développement. Dès lors que les actions des divers secteurs de l'administration se renforcent mutuellement, il en résulte des synergies qui facilitent la réalisation de l'objectif international de réduction de la pauvreté. C'est à cette seule condition que les efforts déployés par les Membres pour faire refluer la pauvreté ne seront pas sapés par les politiques et mesures adoptés dans d'autres domaines au sein de leur administration.

La cohérence des politiques suppose une bonne connaissance des retombées sur la pauvreté des mesures prises dans les différents domaines et la mise en place de mécanismes permettant de résoudre les conflits, ou de concilier des stratégies aux effets contradictoires. Pour œuvrer efficacement à la cohérence de leurs politiques, les organismes d'aide doivent améliorer les mécanismes qui leur permettent de coordonner leur action avec celles d'autres institutions compétentes dans des domaines ayant une influence sur la réduction de la pauvreté (par exemple les échanges, l'agriculture, l'environnement, l'allégement de la dette, les migrations, etc.), qu'il s'agisse d'autres instances de leur administration nationale, d'autres organismes présents dans les pays partenaires ou d'institutions d'envergure mondiale.

Dans le chapitre 4, on a souligné l'importance de la cohérence des politiques et suggéré certaines mesures que les gouvernements Membres du CAD pourraient prendre, sur le plan des procédures, pour l'améliorer. Les organismes bilatéraux ont un rôle fondamental de catalyseur à jouer pour sensibiliser les autres instances de l'administration nationale à l'importance de la cohérence des politiques et faire en sorte qu'une action soit engagée dans ce sens, ce qui ne va pas sans conséquences au niveau de leurs capacités institutionnelles dans quatre domaines essentiels :

Les organismes d'aide doivent se doter des moyens voulus pour étudier le rôle de telle ou telle politique gouvernementale, analyser son impact sur la pauvreté et déterminer à quel niveau, au sein du gouvernement, des ajustements peuvent être apportés afin de rehausser la cohérence. Tout ceci exige du temps et des ressources humaines, et une réflexion éclairée sur les changements que l'on peut raisonnablement escompter. Les organismes d'aide doivent jouer un rôle de catalyseurs et de promoteurs, en reconnaissant que chaque pays poursuit une multitude d'objectifs légitimes et que les décisions sur les questions délicates passent par la mobilisation du soutien des ministères compétents et de l'ensemble du gouvernement.

Il faut établir des mécanismes permettant des communication et des consultations plus étroites avec tout un éventail de ministères, services et organismes afin de remédier aux contradictions et incohérences éventuelles. Une formule qui donne de bons résultats est la création d'une unité centrale de coordination ayant autorité pour établir d'étroites relations entre les différentes instances gouvernementales.

- Il faut renforcer autant que possible la capacité des organismes d'aide d'influer sur les décisions des autres instances afin qu'une place plus importante soit faite à la cohérence des politiques dans l'échelle des priorités nationales et qu'on entreprenne de s'attaquer aux contradictions et incohérences des politiques gouvernementales. Cela nécessite de disposer des compétences voulues pour étudier des problèmes complexes de cohérence des politiques, évaluer les arbitrages 
à opérer et négocier des solutions de compromis, qui tiennent compte des considérations de développement, entre divers services de l'administration et ministères des pays de l'OCDE.

- Une plus grande attention doit être portée à la cohérence interne du système d'aide lui-même, afin de s'assurer que les stratégies et décisions des autres ministères dont relève tel ou tel aspect particulier de l'aide au développement, des organismes d'exécution et des représentants nationaux au sein des instances dirigeantes des institutions multilatérales de développement, sont compatibles avec l'objectif de réduction de la pauvreté.

Mesures à envisager

Les dirigeants doivent rechercher l'appui des pouvoirs publics au plus haut niveau afin d'être sûrs que les autres ministères et services gouvernementaux tiennent compte des considérations de développement dans la formulation de leurs politiques. Ils doivent aussi s'appliquer à rallier le soutien des plus hautes sphères pour le renforcement des mécanismes de coordination avec les autres instances gouvernementales, le règlement des conflits d'intérêts et la mise au point de dispositifs permettant de remédier aux contradictions et incohérences éventuelles.

- Il faut renforcer la capacité du personnel d'appréhender les implications des différentes politiques gouvernementales.

- Il faut faire prendre conscience au personnel de l'importance des questions de cohérence des politiques et encourager les agents à promouvoir une amélioration de la cohérence dans leur sphère d'intervention ou d'influence.

\section{Conclusion}

L'intégration des principes de réduction de la pauvreté, de partenariat et de cohérence des politiques à toutes les décisions et activités des organismes d'aide appelle l'adaptation des structures institutionnelles, des systèmes et des pratiques de gestion à ces objectifs. Cependant, le changement institutionnel ne doit pas se limiter aux services de l'organisme d'aide. Il doit aussi concerner les interactions entre les organismes bilatéraux car l'efficacité de l'aide et le partenariat sont assujettis à une intensification de la coopération et de la collaboration. Chaque organisme doit donc s'appliquer à mieux connaître le mode de fonctionnement de ses homologues, de façon à découvrir les similitudes et les chevauchements d'activités qui peuvent exister. Il lui sera ainsi possible d'étudier comment renforcer les synergies et les complémentarités, de tirer parti des atouts des autres organismes, et d'opérer un partage des tâches.
Le changement institutionnel ne doit pas se limiter au fonctionnement interne des organismes d'aide; il doit aussi modifier les interactions entre les organismes d'aide de manière à permettre entre ces derniers un partenariat plus étroit, à renforcer les synergies et à faciliter l'exploitation des atouts respectifs. 


\section{Notes}

1. «Intégrer » le principe de la réduction de la pauvreté suppose de faire de cet élément un axe essentiel des processus de formulation des politiques, de planification, de mise en œuvre, d'évaluation et de prise des décisions.

2. Ce chapitre 5 s'appuie sur les résultats d'un atelier spécial organisé par le POVNET en février 2001 au cours duquel ont été examinées les questions liées à la réorganisation des structures institutionnelles et auquel ont participé des hauts responsables des organismes d'aide ainsi que des agents de terrain et des responsables de la gestion des ressources humaines.

3. Cette action, décrite dans les chapitres 1 à 4 des Lignes directrices, passe par l'instauration de partenariats avec d'autres acteurs du développement afin d'appuyer des stratégies de réduction de la pauvreté définies et pilotées par les pays intéressés eux-mêmes.

4. Les systèmes de gestion des ressources humaines sont un aspect essentiel des dispositifs organisationnels et comprennent divers éléments dont la rémunération, les avantages, les mesures d'incitation, la gestion des performances, la définition des postes, le recrutement, les perspectives de carrière et la formation.

5. Les 25 études de cas sur lesquelles repose l'étude approfondie du CAD (DAC Scoping Study of Donor Poverty Reduction Policies and Practices) en date de 1999 offrent de nombreux exemples des changements apportés par les organismes d'aide à leurs structures.

6. Les cases d'un organigramme indiquent i) la manière dont sont groupées les personnes pour assurer un fonctionnement efficace de l'organisation, et ii) les relations hiérarchiques, qui correspondent généralement à une hiérarchie des responsabilités et des fonctions. Les «blancs » représentent les articulations qui s'établissent au-dessus, au-dessous et de chaque côté de ces cases et qui transcendent les cloisonnements fonctionnels - et permettent véritablement de mener à bien le travail de l'organisation, par exemple dans le cas où une stratégie émanant d'un service du siège est transmise à une équipe qui devra en assurer la mise en œuvre sur le terrain. Les «blancs » correspondent aussi aux pratiques de gestion et aux systèmes de gestion des ressources humaines qui guident et motivent le personnel et qui, à un autre niveau, transcendent aussi les cloisonnements fonctionnels et exercent une influence sur la manière dont est accompli le travail de l'organisme. 


\section{Appendice : Objectifs de développement du millénaire (ODM)}

\section{OBJECTIF 1. ÉLIMINER L'EXTRÊME PAUVRETÉ ET LA FAIM}

Cible 1. Réduire de moitié, entre 1990 et 2015, la proportion de la population dont le revenu est inférieur à un dollar par jour

Cible 2. Réduire de moitié, entre 1990 et 2015, la proportion de la population qui souffre de la faim

\section{OBJ ECTIF 2. ASSURER UNE ÉDUCATION PRIMAIRE POUR TOUS}

Cible 3. D'ici à 2015, donner à tous les enfants, garçons et filles, partout dans le monde, les moyens d'achever un cycle complet d'études primaires
1. Proportion de la population disposant de moins d'un dollar par jour

2. Indice d'écart de la pauvreté [incidence de la pauvreté x degré de pauvreté]

3. Part du cinquième le plus pauvre de la population dans la consommation nationale

4. Pourcentage d'enfants de moins de 5 ans présentant une insuffisance pondérale

5. Proportion de la population n'atteignant pas le niveau minimal d'apport calorique

6. Taux net de scolarisation dans le primaire

7. Proportion d'écoliers commençant la première année d'études dans l'enseignement primaire et achevant la cinquième

8. Taux d'alphabétisation des 15 à 24 ans

\section{OBJECTIF 3. PROMOUVOIR L'ÉGALITÉ DES SEXES ET L'AUTONOMISATION DES FEMMES}

Cible 4. Éliminer les disparités entre les sexes dans les enseignements primaire et secondaire d'ici à 2005 si possible, et à tous les niveaux de l'enseignement en 2015 au plus tard

9. Rapport filles/garçons dans l'enseignement primaire, secondaire et supérieur

10. Taux d'alphabétisation des femmes de 15 à 24 ans par rapport aux hommes

11. Pourcentage de femmes salariées dans le secteur non agricole

12. Proportion de sièges occupés par des femmes au parlement national

\section{OBJ ECTIF 4. RÉDUIRE LA MORTALITÉ DES ENFANTS DE MOINS DE 5 ANS}

Cible 5. Réduire de deux tiers, entre 1990 et 2015, le taux de mortalité des enfants de moins de 5 ans

13. Taux de mortalité des enfants de moins de 5 ans

14. Taux de mortalité infantile

15. Proportion d'enfants de 1 an vaccinés contre la rougeole

\section{OB) ECTIF 5. AMÉLIORER LA SANTÉ M ATERNELLE}

Cible 6. Réduire de trois quarts, entre 1990 et 2015, le taux

16. Taux de mortalité maternelle

de mortalité maternelle

17. Proportion d'accouchements assistés par du personnel de santé qualifié 


\section{Appendice : Objectifs de développement du millénaire (ODM)}

\section{OBJ ECTIFS ET CIBLES}

INDICATEURS

\section{OBJ ECTIF 6. COMBATTRE LE VIH/ SIDA, LE PALUDISME ET \\ D'AUTRES MALADIES}

Cible 7. D'ici à 2015, avoir stoppé la propagation du VIH/sida et commencé à inverser la tendance actuelle

Cible 8. D'ici à 2015, avoir maîtrisé le paludisme et d'autres grandes maladies, et avoir commencé à inverser la tendance actuelle
18. Taux de prévalence du VIH parmi les femmes enceintes âgées de 15 à 24 ans

19. Taux d'utilisation de la contraception

20. Nombre d'enfants orphelins du sida

21. Taux de prévalence du paludisme et taux de mortalité lié à cette maladie

22. Proportion de la population vivant dans les zones à risque qui utilisent des moyens de protection et des traitements efficaces contre le paludisme

23. Taux de prévalence de la tuberculose et taux de mortalité lié à cette maladie

24. Proportion de cas de tuberculose détectés et soignés dans le cadre de traitements de brève durée sous surveillance directe

\section{OBJECTIF 7. ASSURER UN ENVIRONNEMENT DURABLE*}

Cible 9. Intégrer les principes du développement durable dans les politiques nationales et inverser la tendance actuelle à la déperdition des ressources environnementales
25. Proportion de zones forestières

26. Superficie des terres protégées pour préserver la biodiversité

27. PIB par unité d'énergie consommée (rendement énergétique)

28. Émissions de dioxyde de carbone (par habitant) [À ces indicateurs s'ajoutent deux chiffres concernant la pollution atmosphérique mondiale : l'appauvrissement de la couche d'ozone et l'accumulation de gaz favorisant le réchauffement de la planète]

Cible 10. Réduire de moitié, d'ici à 2015, le pourcentage de la population qui n'a pas accès de façon durable à un approvisionnement en eau potable salubre

Cible 11. Réussir, d'ici à 2020, à améliorer sensiblement la vie d'au moins 100 millions d'habitants de taudis
29. Proportion de la population ayant accès à une source d'eau meilleure

30. Proportion de la population ayant accès à un meilleur système d'assainissement

31. Proportion de la population ayant accès à la sécurité d'occupation des logements

[La ventilation urbaine/rurale de plusieurs des indicateurs ci-dessus pourra être utile au suivi de l'amélioration de la situation des habitants de taudis] 


\section{Appendice : Objectifs de développement du millénaire (ODM)}

\section{OBJ ECTIF 8. METTRE EN PLACE UN PARTENARIAT MONDIAL POUR LE DÉVELOPPEMENT*}

Cible 12. Poursuivre la mise en place d'un système commercial et financier multilatéral ouvert, fondé sur des règles, prévisible et non discriminatoire

[Cela suppose un engagement en faveur d'une bonne gouvernance, du développement et de la lutte contre la pauvreté, aux niveaux tant national qu'international]

Cible 13. S'attaquer aux besoins particuliers des pays les moins avancés

[La réalisation de cette cible suppose l'admission en franchise et hors contingents des produits exportés par les pays les moins avancés ; l'application du programme renforcé d'allégement de la dette des PPTE et l'annulation des dettes publiques bilatérales; et l'octroi d'une APD plus généreuse aux pays qui démontrent leur volonté de lutter contre la pauvreté]

Cible 14. Répondre aux besoins particuliers des petits États insulaires en développement

[en appliquant le Programme d'action pour le développement durable des petits États insulaires en développement et les conclusions de la vingt-deuxième session extraordinaire de l'Assemblée générale]

Cible 15. Traiter globalement le problème de la dette des pays en développement, par des mesures d'ordre national et international propres à rendre leur endettement viable à long terme
Certains des indicateurs ci-après seront évalués séparément dans les cas des pays les moins avancés (PMA), de l'Afrique, des pays sans littoral et des petits États insulaires en développement

\section{Aide publique au développement}

32. APD nette, en pourcentage du revenu national brut des pays donateurs (cibles : $0,7 \%$ au total et $0,15 \%$ pour les PMA)

33. Proportion de l'APD consacrée aux services sociaux de base (éducation de base, soins médicaux primaires, nutrition, eau salubre et assainissement)

34. Proportion de l'APD qui est déliée

35. Proportion de l'APD consacrée à l'environnement dans les petits États insulaires en développement

36. Proportion de l'APD consacrée au secteur des transports dans les pays sans littoral

\section{Accès au marché}

37. Proportion des exportations (en valeur et à l'exclusion des armes) admises en franchise de droits de douane et hors contingents

38. Taux moyens de droits de douane et contingents appliqués aux produits agricoles, textiles et vêtements

39. Subventions agricoles nationales et à l'exportation dans les pays de l'OCDE

40. Proportion de l'APD allouée au renforcement des capacités commerciales

\section{Viabilité de la dette}

41. Proportion de la dette publique bilatérale des PPTE qui a été annulée

42. Service de la dette, en pourcentage des exportations de biens et services

43. Proportion de l'APD fournie au titre de l'allégement de la dette

44. Nombre de pays ayant atteint les points de décision et d'achèvement de l'Initiative PPTE 


\section{Appendice : Objectifs de développement du millénaire (ODM)}

OBJ ECTIFS ET CIBLES

OBJ ECTIF 8. METTRE EN PLACE UN PARTENARIAT MONDIAL

Cible 16. En coopération avec les pays en développement, formuler et appliquer des stratégies qui permettent aux jeunes de trouver un travail décent et utile

Cible 17. En coopération avec l'industrie pharmaceutique, rendre les médicaments essentiels disponibles et abordables dans les pays en développement

Cible 18. En coopération avec le secteur privé, faire en sorte que tous bénéficient des avantages des nouvelles technologies, en particulier des technologies de l'information et de la communication,

\section{INDICATEURS}

45. Taux de chômage des 15 à 24 ans

46. Proportion de la population ayant durablement accès à des médicaments de base d'un coût abordable

47. Nombre de lignes téléphoniques pour 1000 habitants

48. Nombre de micro-ordinateurs pour 1000 habitants

* Les indicateurs retenus pour les objectifs 7 et 8 sont à préciser 


\section{Lignes directrices du CAD}

Le Comité d'aide au développement de l'OCDE adopte des orientations destinées à guider la conduite par ses Membres de leurs programmes de coopération pour le développement. Ces lignes directrices reflètent les vues des Membres et les enseignements qui se dégagent de leur expérience et font appel à la contribution d'institutions multilatérales et d'experts indépendants, y compris d'experts de pays en développement.

\section{Le rôle de la coopération pour le développement à l'aube du XXI' siècle}

Le rapport sur Le rôle de la coopération pour le développement à l'aube du XXI siècle approuvé par le CAD à sa réunion à haut niveau de 1996 définit les orientations stratégiques de la coopération pour le développement pour le $\mathrm{XXI}^{\mathrm{e}}$ siècle. Il rappelle l'importance du développement pour les populations du monde entier et les avancées impressionnantes qui ont été obtenues sur le plan du développement humain au cours des cinquante dernières années. Il propose un projet d'avenir s'articulant autour d'un ensemble d'objectifs - concernant le bien-être économique, le développement social et la durabilité environnementale - dérivés des résultats des conférences des Nations unies et préconise, pour donner corps à ce projet, des stratégies fondées sur le partenariat destinées à favoriser l'effort propre, une amélioration de la coordination et la cohérence des politiques. Les objectifs, et l'approche fondée sur le partenariat, qui y sont présentés ont depuis lors rallié l'adhésion de l'ensemble de la communauté internationale du développement.

Dans ces conditions, les Membres du CAD ont entrepris d'élaborer une série de lignes directrices à l'appui des ambitieux objectifs définis dans Le rôle de la coopération pour le développement à l'aube du XXI siècle.

Les lignes directrices du CAD (2001):

- La réduction de la pauvreté

- Stratégies de développement durable

- Renforcer les capacités commerciales au service du développement

- Prévenir les conflits violents : quels moyens d'action?

\section{Ouvrages déjà parus dans la série des Lignes directrices du CAD}

- Lignes directrices du CAD pour l'égalité homme-femme et le renforcement du pouvoir des femmes dans le cadre de la coopération pour le développement

- L'expansion du secteur privé

- Le développement participatif et la bonne gestion des affaires publiques

- L'aide des donneurs en faveur du développement des capacités dans le domaine de l'environnement

- Lignes directrices sur l'aide et l'environnement

$\mathrm{N}^{\circ} 1$ : Bonnes pratiques pour les études de l'impact sur l'environnement exercé par les projets de développement

$\mathrm{N}^{\circ} 2$ : Bonnes pratiques pour les études et stratégies nationales concernant l'environnement

$\mathrm{N}^{\circ} 3$ : Lignes directrices établies à l'intention des organismes d'aide concernant le déplacement involontaire et la réinstallation dans le cadre de projets de développement

$\mathrm{N}^{\circ} 4$ : Lignes directrices sur les problèmes d'environnement de dimension globale destinées aux organismes d'aide

$N^{\circ} 5$ : Lignes directrices établies à l'intention des organismes d'aide relatives à la gestion des produits chimiques

$\mathrm{N}^{\circ} 6$ : Lignes directrices établies à l'intention des organismes d'aide pour la lutte contre les parasites et la gestion des pesticides

$N^{\circ} 7$ : Lignes directrices établies à l'intention des organismes d'aide relatives à la réduction de l'incidence des catastrophes naturelles

$\mathrm{N}^{\circ} 8$ : Lignes directrices établies à l'intention des organismes d'aide concernant les aspects régionaux et mondiaux de la mise en valeur et de la protection des milieux marins et littoraux

$N^{\circ} 9$ : Lignes directrices établies à l'intention des organismes d'aide pour une meilleure préservation et une utilisation durable des zones humides tropicales et subtropicales

\section{Consultez le site web du CAD/OCDE à l'adresse ww w.oecd.org/ dac}


LES ÉDITIONS DE L'OCDE, 2, rue André-Pascal, 75775 PARIS CEDEX 16 IMPRIMÉ EN FRANCE

(43 2001092 P) ISBN 92-64-29506-2 - n 521042001 